LA-14393

Approved for public release;

distribution is unlimited.

Structural Health Monitoring Algorithm Comparisons Using Standard Data Sets
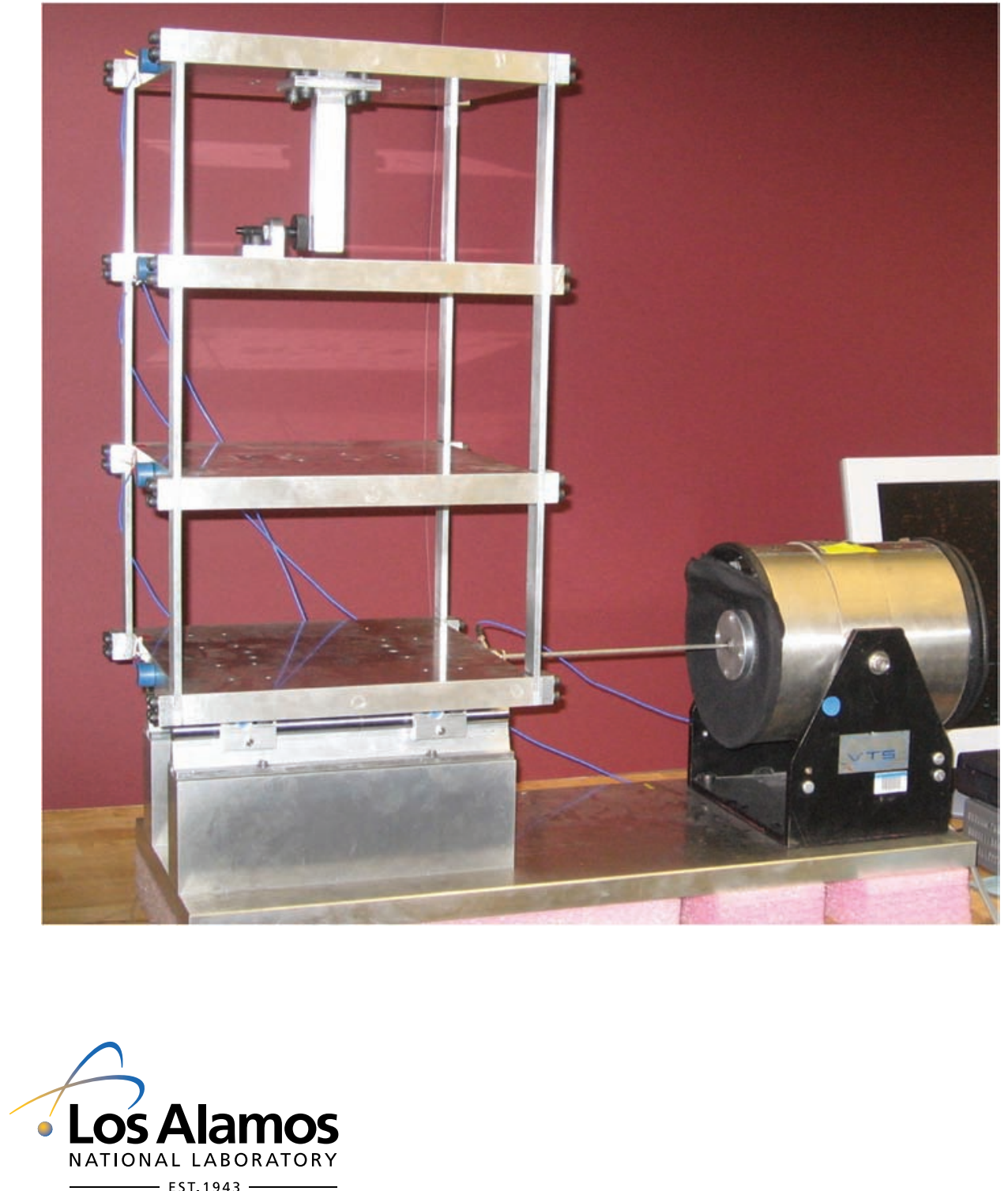
Edited by Mable Amador, Group IRM-CAS.

Photocomposition by Deidre' A. Plumlee, Group IRM-CAS.

About the cover: An aluminum frame structure used as a standard structural health monitoring test system.

Los Alamos National Laboratory, an affirmative action/ equal opportunity employer, is operated by Los Alamos National Security, LLC, for the National Nuclear Security Administration of the U.S. Department of Energy under contract DE-AC52-06NA25396.

This report was prepared as an account of work sponsored by an agency of the U.S. Government. Neither Los Alamos National Security, LLC, the U.S. Government nor any agency thereof, nor any of their employees make any warranty, express or implied, or assume any legal liability or responsibility for the accuracy, completeness, or usefulness of any information, apparatus, product, or process disclosed, or represent that its use would not infringe privately owned rights. Reference herein to any specific commercial product, process, or service by trade name, trademark, manufacturer, or otherwise does not necessarily constitute or imply its endorsement, recommendation, or favoring by Los Alamos National Security, LLC, the U.S. Government, or any agency thereof. The views and opinions of authors expressed herein do not necessarily state or reflect those of Los Alamos National Security, LLC, the U.S. Government, or any agency thereof. Los Alamos National Laboratory strongly supports academic freedom and a researcher's right to publish; as an institution, however, the Laboratory does not endorse the viewpoint of a publication or guarantee its technical correctness. 
LA-14393

Issued: March 2009

\section{Structural Health Monitoring Algorithm}

Comparisons Using Standard Data Sets

Elói Figueiredo*

Gyuhae Park

Joaquim Figueiras*

Charles Farrar

Keith Worden** 
This page left blank intentionally. 


\section{TABLE OF CONTENTS}

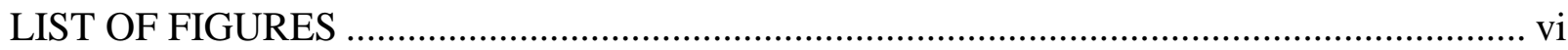

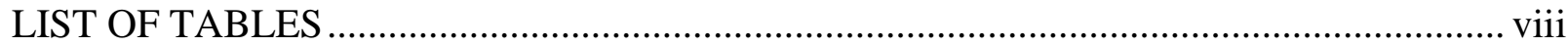

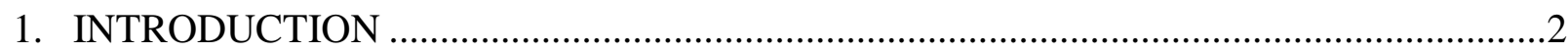

1.1 The Structural Health Monitoring Process ...................................................................2

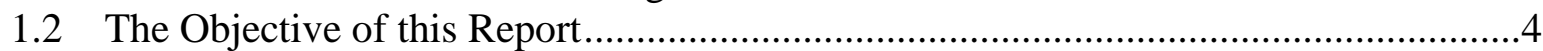

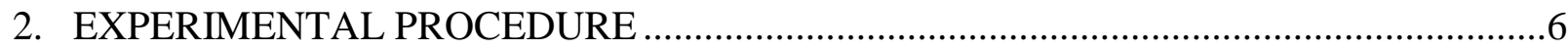

2.1 Test Structure Description ..............................................................................

2.2 Data Acquisition System …………..................................................................

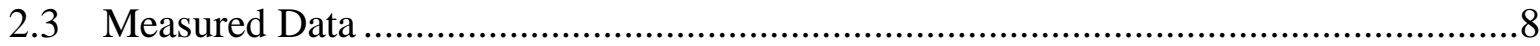

2.3.1 Operational and Environmental Effects...........................................................11

2.3.2 Nonlinearities Effects........................................................................12

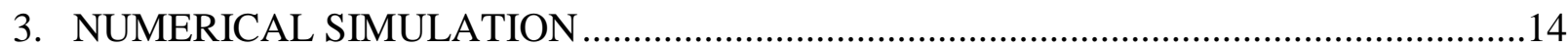

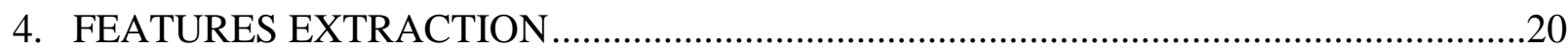

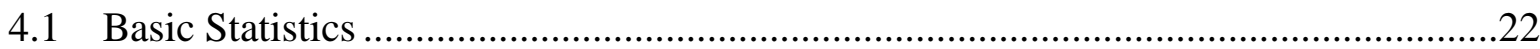

4.1.1 First Four Statistical Moments of the Measured Data ...................................22

4.1.2 Probability Density Distribution ..................................................................26

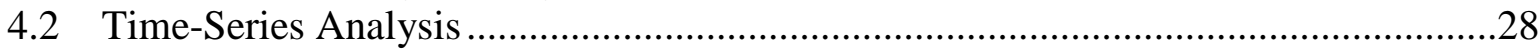

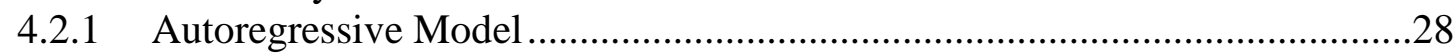

4.2.1.1 Model Identification .....................................................................29

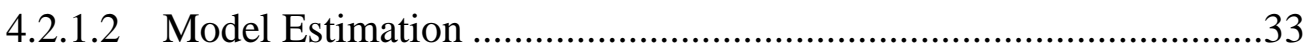

4.2.2 Principal Component Analysis of Autoregressive Parameters .........................43

4.2.3 Correlation Coefficient Using Time Domain Data .........................................45

$4.3 \quad$ Frequency Domain Analysis................................................................................

4.3.1 Modal Parameters .....................................................................................4

4.3.2 Correlation Coefficients Using Frequency Response Functions ......................51

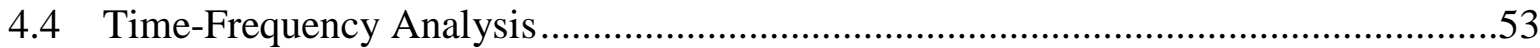

4.4.1 Short-Time Fourier Transform .................................................................53

4.4.2 Wavelet Transform ..............................................................................59

4.4.3 Holder Exponent ...........................................................................................63

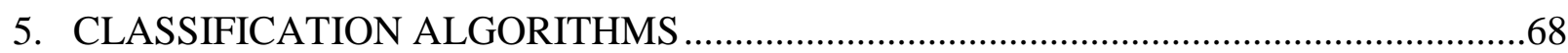

5.1 Statistical Process Control .......................................................................................68

5.1.1 Statistical Basis of the Shewhart X-bar Control Charts..................................69

5.1.2 Control Charts with Autocorrelated Process Data ..............................................70

5.1.3 Control Chart Analysis ...............................................................................70

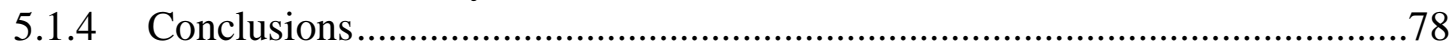

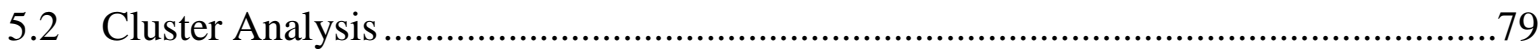

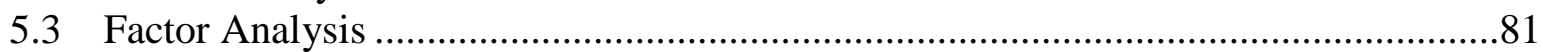

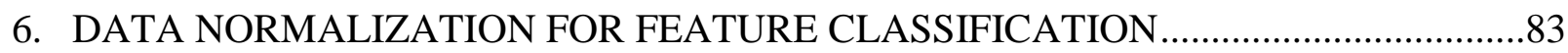

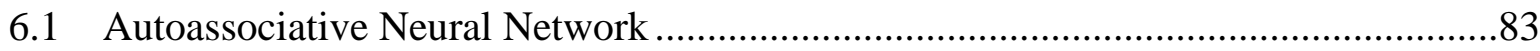

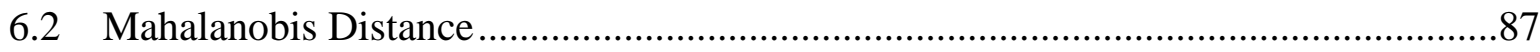

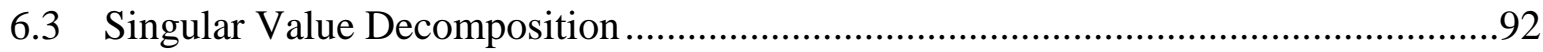

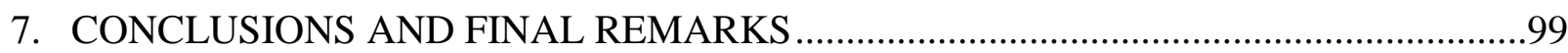

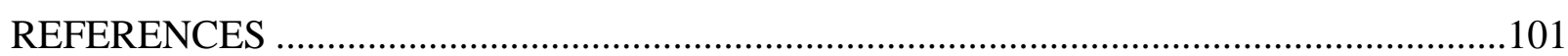




\section{LIST OF FIGURES}

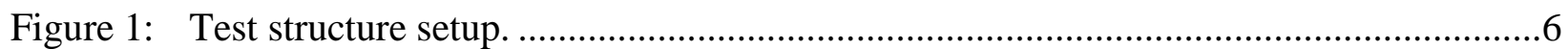

Figure 2: Basic dimensions of the three-story building structure (all dimensions are in $\mathrm{cm}$ ).......7

Figure 3: Structural details. ........................................................................................9

Figure 4: Force-time history from Channel 1 of State \#1. ................................................... 10

Figure 5: Acceleration-time history from Channel 5 of State \#1..........................................11

Figure 6: FRF and coherence function from Channel 5 of State \#1.........................................11

Figure 7: FRFs (based on five averages) of the response at Channel 5 and the excitation force at Channel 1 for State \#1 (baseline) and State \#3 (1.2-kg added mass on the first floor) ............................................................................................... 12

Figure 8: FRFs (based on five averages) of the response at Channel 5 and the excitation force at Channel 1 for State \#1 (baseline), State \#14 (gap = $0.05 \mathrm{~mm}$ ), and State \#17 (gap $=0.10 \mathrm{~mm}$ and $1.2-\mathrm{kg}$ added mass on the first floor).

Figure 9: Shear-building model of the test structure.............................................................14

Figure 10: Numerical (NM) and experimental (Exp) mode shapes of the baseline condition......16

Figure 11: Correlation between numerical and experimental mode shapes by MAC..................16

Figure 12: Experimental and numerical responses of the baseline condition from Channel 5, resulting from the measured experimental excitation at Channel 1 ............................17

Figure 13: Autocorrelation functions of the experimental and numerical responses...................18

Figure 14: PSD of the experimental and numerical time histories..........................................19

Figure 15: Mean for all time histories from Channel 5 of all state conditions.............................20

Figure 16: Standard deviation for all time histories from Channel 5 of all state conditions.........21

Figure 17: Skewness for all time histories from Channel 5 of all state conditions. .....................21

Figure18: Kurtosis for all time histories from Channel 5 of all state conditions. ......................21

Figure 19: Autocorrelation functions for all time histories from Channel 5 of State \#1 and

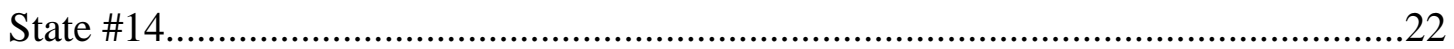

Figure 20: Acceleration-time histories of various state conditions from Channels 2 to 5...........23

Figure 21: First four statistical moments for each state condition. ............................................25

Figure 22: Normal probability plots of four state conditions for Channel 5..............................27

Figure 23: PDFs estimated by a kernel density estimator using time histories from

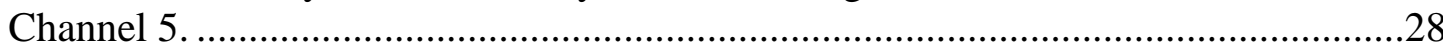

Figure 24: AIC values of AR models of increasing order (Channel 5 of State \#1). ....................30

Figure 25: Estimated PAF of AR models of increasing order (Channel 5 of State \#1). ...............31

Figure 26: RMSE of AR models of increasing order (Channel 5 of State \#1).............................32

Figure 27: SVD of the AR(60) model using a time history from Channel 5 of State \#1..............34

Figure 28: AR(5) model parameters for all structural state conditions (Channel 5)....................34

Figure 29: AR(30) model parameters for all structural state conditions (Channel 5)...................34

Figure 30: Amplitude of the $3^{\text {rd }}$ parameter from the AR(5) model at Channel 5 for all state

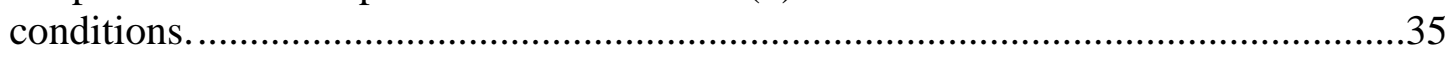

Figure 31: Comparison of the measured and estimated time histories using the AR(5) model fit to State \#1 data from Channel 5................................................................35

Figure 32: Comparison of the measured and estimated time histories using the AR(30) model fit to State \#1 data from Channel 5 ...........................................................36

Figure 33: AR(5) residual errors histograms with the corresponding Gaussian distribution

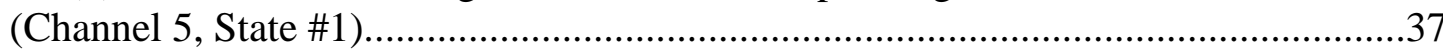

Figure 34: AR(30) residual errors histograms with the corresponding Gaussian distribution

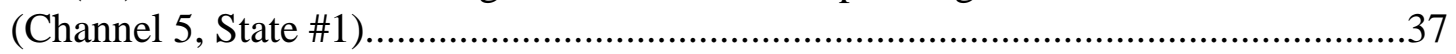

Figure 35: Log PSD of the AR(5) model residual errors (Channel 5, State \#1)........................38 
Figure 36: Log PSD of the AR(30) model residual errors (Channel 5, State \#1). ......................38

Figure 37: Autocorrelation functions of the original time histories from Channel 5..................39

Figure 38: Autocorrelation functions of the AR(5) residual errors.........................................40

Figure 39: Autocorrelation functions of the AR(30) residual errors...........................................40

Figure 40: Lag plots of the original time history from Channel 5............................................41

Figure 41: Lag plots of the AR(5) model residual errors from Channel 5 ...............................42

Figure 42: Lag plots of the AR(30) model residual errors from Channel 5.............................42

Figure 43: Plot of the percentage variability explained by each principal component

(Channel 5).

Figure 44: AR(30) parameters (Channel 5) projected onto the first two principal components...45

Figure 45: Plot of the variability explained by the first principal component of each state

(Channel 5)

Figure 46: Correlation coefficient, $r_{i j}$, by state condition, between Channels $i$ and $j$..............47

Figure 47: CMIF for one FRF from Channel 5 of State \#1......................................................49

Figure 48: Curve fitting example for the FRFs from Channels 2-5 of State \#1........................49

Figure 49: Natural frequencies for all state conditions. ........................................................50

Figure 50: Frequency deviations from the baseline condition (State \#1) for all state conditions.

Figure 51: Correlation coefficient per state condition across the channels...............................52

Figure 52: Correlation coefficient per state condition.......................................................53

Figure 53: STFT analysis of signal from State \#1, Channel 4. .................................................55

Figure 54: STFT analysis of signal from State \#10, Channel 4. ...............................................56

Figure 55: STFT analysis of signal from State \#14, Channel 4. .................................................57

Figure 56: Individual spectrograms in concatenated format, Channel 4....................................58

Figure 57: CWT of time history from State \#1, Channel 4 ...................................................60

Figure 58: CWT of time history from State \#10, Channel 4...................................................61

Figure 59: CWT of time history from State \#14, Channel 4 ...................................................62

Figure 60: Details of the CWT for the time histories from State \#1(a) and State \#14 (b), Channel 4.

Figure 61: AR(30) model residual errors of one time history from Channel 4 of State \#10.........65

Figure 62: Details of the CWT for the time history from State \#10, Channel 4. ........................65

Figure 63: Portion of a time history from State \#10 (Channel 4) with the associated Holder exponent function and residual errors from both AR models.

Figure 64: The X-Bar control plot of the mean of the grouped AR(5) residual errors

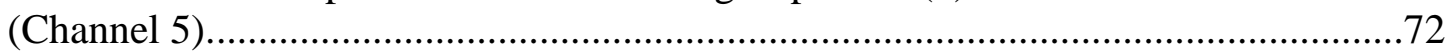

Figure 65: The X-Bar control plot of the mean of the grouped AR(5) residual errors (Channel 5)

Figure 66: The X-Bar control plot of the mean of the grouped AR(5) residual errors (Channel 5) .74

Figure 67: The X-Bar control plot of the mean of the grouped AR(30) residual errors (Channel 5) .75

Figure 68: The X-Bar control plot of the mean of the grouped AR(30) residual errors (Channel 5)

Figure 69: The X-Bar control plot of the mean of the grouped AR(30) residual errors (Channel 5)

Figure 70: Number of outliers falling outside the control limits. The horizontal dashed line corresponds to the maximum number of outliers found in the undamaged states (Channel 5)

Figure 71: Traditional representation of the Hierarchical Clustering. .80 
Figure 72: Feature classification assuming two clusters using AR(30) parameters

(Channel 5).

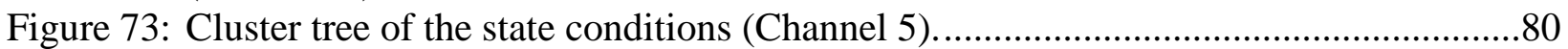

Figure 74: FA schematic model (Kullaa, 2003) ....................................................................82

Figure 75: Factor loadings of each state condition (Channel 5) ..............................................82

Figure 76: Specific variance by state condition (Channel 5). ...............................................83

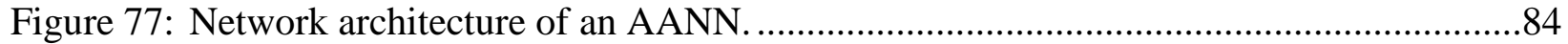

Figure 78: Output in the bottleneck layer and measured data (Signals 1 and 2, Temperature). ...86

Figure 79: Residual errors using the AR(5) model parameters estimated at Channel 5..............86

Figure 80: Novelty indices using the AR(5) model parameters estimated at Channel 5. ............86

Figure 81: Mahalanobis squared distances using the AR(5) parameters (Channel 5). ................88

Figure 82: Mahalanobis squared distances using the AR(30) parameters (Channel 5). ..............88

Figure 83: Mahalanobis squared distance for outlier detection using the AR(5) parameters estimated from Channel 5............................................................................8

Figure 84: Mahalanobis squared distance for outlier detection using the AR(30) parameters estimated from Channel 5.

Figure 85: Mahalanobis squared distance for undamaged and damaged states using AR(5) grouped residual errors. The dash lines, CV1 and CV2, are the threshold lines.........90

Figure 86: Mahalanobis squared distance for undamaged and damaged states using AR(30) grouped residual errors. The dash lines, CV1 and CV2, are the threshold lines.........90

Figure 87: FRFs of five state conditions estimated at Channel 4.........................................93

Figure 88: First 15 singular values in decreasing order for Channels 2-5 (State \#1). ..................94

Figure 89: First 15 singular values in decreasing order for Channels 2-5 (State \#3). ..................95

Figure 90: First 15 singular values in decreasing order for Channels 2-5 (State \#7). ..................96

Figure 91: First 15 singular values in decreasing order for Channels 2-5 (State \#14)................97

Figure 92: First 15 singular values in decreasing order for Channels 2-5 (State \#17)................98

\section{LIST OF TABLES}

Table 1: Characteristics of the Sensors Used Throughout the Testing ..................................... 8

Table 2: $\quad$ Data Labels of the Structural State Conditions........................................................ 9

Table 3: Experimental and Numerical Modal Parameters ................................................... 16

Table 4: Experimental Natural Frequencies and Damping Ratios of All State Conditions...... 50 


\section{NOTATION}

All symbols used in this report are defined when they first appear in the text. For the reader's convenience, this section contains the principal meanings of the commonly used acronyms. Some symbols denote more than one meaning, but their meaning should be clear when read in context.

\section{ABBREVIATIONS}

AANN

AIC

$\operatorname{AR}(p)$

CMIF

CWT

DOF

EI

FA

FEUP

FRF

LABEST

LANL

MAC

NLPCA

PAF

PCA

PDF

PSD

RFP

RMSE

SHM

SPC

STFT

SVD

UCSD

WT
Autoassociative neural network

Akaike's information criterion

Autoregressive model of order $p$

Complex mode indicator function

Continuous wavelet transform

Degree-of-freedom

Engineering Institute

Factor analysis

Faculty of Engineering of the University of Portugal

Frequency response function

Laboratory for Concrete Technology and Structural Behavior

Los Alamos National Laboratory

Modal assurance criterion

Nonlinear principal components analysis

Partial autocorrelation function

Principal components analysis

Probability density function

Power spectral density

Rational-fraction polynomial

Root mean squared error

Structural health monitoring

Statistical process control

Short-time Fourier transform

Singular value decomposition

University of California, San Diego

Wavelet transform 


\begin{tabular}{ll}
\multicolumn{2}{c}{ Roman Symbols } \\
$E[]$ & Wavelet transform coefficient \\
$i$ & Expected value of [] \\
$n$ & Index \\
$N$ & Sample size \\
$p$ & Population size \\
$r$ & Number of parameters of the autoregressive model \\
$R_{X X}$ & Sample correlation coefficient \\
$R_{X y}$ & Autocorrelation function \\
$s$ & Cross-correlation function \\
$s^{2}$ & Sample standard deviation \\
$t$ & Sample variance \\
$\bar{X}$ & Time variable \\
$X$ & Sample average of $X$ \\
$\bar{y}$ & Random variable \\
$Y$ & Sample average of $Y$ \\
& Random variable \\
&
\end{tabular}

Greek Symbols

$\mu \quad$ Population mean

$\rho \quad$ Correlation coefficient

$\Sigma \quad$ Covariance matrix

$\sigma \quad$ Population standard deviation

$\sigma^{2} \quad$ Population variance

$\tau \quad$ Time lag 


\title{
STRUCTURAL HEALTH MONITORING ALGORITHM COMPARISONS USING STANDARD DATA SETS
}

by

Elói Figueiredo, ${ }^{1}$ Gyuhae Park, ${ }^{2}$ Joaquim Figueiras, ${ }^{1}$ Charles Farrar, ${ }^{2}$ and Keith Worden ${ }^{3}$

\begin{abstract}
The real-world structures are subjected to operational and environmental condition changes that impose difficulties in detecting and identifying structural damage. The aim of this report is to detect damage with the presence of such operational and environmental condition changes through the application of the Los Alamos National Laboratory's statistical pattern recognition paradigm for structural health monitoring (SHM). The test structure is a laboratory three-story building, and the damage is simulated through nonlinear effects introduced by a bumper mechanism that simulates a repetitive impact-type nonlinearity. The report reviews and illustrates various statistical principles that have had wide application in many engineering fields. The intent is to provide the reader with an introduction to feature extraction and statistical modelling for feature classification in the context of SHM. In this process, the strengths and limitations of some actual statistical techniques used to detect damage in the structures are discussed. In the hierarchical structure of damage detection, this report is only concerned with the first step of the damage detection strategy, which is the evaluation of the existence of damage in the structure. The data from this study and a detailed description of the test structure are available for download at: http://institute.lanl.gov/ei/software-and-data/.
\end{abstract}

${ }^{1}$ Department of Civil Engineering, Faculty of Engineering of the University of Porto, Porto, Portugal.

${ }^{2}$ The Engineering Institute, Los Alamos National Laboratory, Los Alamos, New Mexico, USA.

${ }^{3}$ Department of Mechanical Engineering, University of Sheffield, Sheffield, UK. 


\section{INTRODUCTION}

This report summarizes the analysis of test data obtained from a laboratory three-story structure as part of structural health monitoring (SHM) process. This research has been performed in the Engineering Institute (EI) at Los Alamos National Laboratory (LANL), in collaboration with the Laboratory for Concrete Technology and Structural Behavior (LABEST) of the Faculty of Engineering of the University of Porto (FEUP). The EI is an education- and research-focused collaboration between the LANL and the University of California, San Diego (UCSD), Jacobs School of Engineering. The technical focus of this institute is damage prognosis, a multidisciplinary engineering science concerned with assessing the current condition and predicting the remaining life of aerospace, civil, and mechanical infrastructures. The LABEST is a research unit that aims to develop innovative technology in the field of material and structural behavior of civil engineering infrastructure. In the last years, this laboratory has gained significant experience in structural monitoring of civil infrastructure.

The aim of the present report is to use LANL's statistical pattern recognition paradigm for SHM on the data set obtained from a laboratory test structure, in order to point out the strengths and limitations of several statistical procedures for detecting damage in the structures.

\subsection{The Structural Health Monitoring Process}

SHM is the process of detecting damage in structures. The goal of SHM is to improve the safety and reliability of aerospace, civil, and mechanical infrastructure by detecting damage before it reaches a critical state. To achieve this goal, technology is being developed to replace qualitative visual inspection and time-based maintenance procedures with more quantifiable and automated damage assessment processes. These processes are implemented using both hardware and software with the intent of achieving more cost-effective condition-based maintenance. A more detailed general discussion of SHM can be found in (Worden et al., 2004; Farrar and Worden, 2007)

The authors believe that all approaches to SHM as well as all traditional nondestructive evaluation procedures (e.g., ultrasonic inspection, acoustic emissions, active thermography) can be cast in the context of a statistical pattern recognition problem (Farrar et al., 2001). The LANL statistical pattern recognition paradigm for the development of SHM solutions can be described as the following four-step process:

i. Operational evaluation;

ii. Data acquisition;

iii. Feature extraction; and

iv. Statistical modelling for feature classification.

A necessary first step to developing an SHM capability is to perform an operational evaluation. This part of the SHM solution process attempts to answer four questions regarding the implementation of a SHM system: (1) What are the life safety and/or economic justifications for monitoring the structure? (2) How is damage defined for the system being monitored? (3) What are the operational and environmental conditions under which the system of interest functions? (4) What are the limitations on acquiring data in the operational environment? Operational evaluation defines, and to the greatest extent possible quantifies, the damage that is to be detected. It also defines the benefits to be gained from deployment of the SHM system. This process also begins to set limitations on what will be monitored and how to perform the 
monitoring as well as tailoring the monitoring to unique aspects of the system and unique features of the damage that is to be detected.

The data-acquisition portion of the SHM process involves selecting the excitation methods; the sensor types, numbers, and locations; and the data acquisition/storage/ processing/transmittal hardware. The actual implementation of this portion of the SHM process will be application specific. A fundamental premise regarding data acquisition and sensing is that these systems do not measure damage. Rather, they measure the response of a system to its operational and environmental loading or the response to inputs from actuators embedded with the sensing system. Depending on the sensing technology deployed and the type of damage to be identified, the sensor readings may be more or less directly correlated to the presence and location of damage. Data-interrogation procedures (feature extraction and statistical modelling for feature classification) are the necessary components of an SHM system that converts the sensor data into information about the structural condition. Furthermore, to achieve successful SHM, the data-acquisition system will have to be developed in conjunction with these datainterrogation procedures.

A damage-sensitive feature is some quantity extracted from the measured systemresponse data that is correlated with the presence of damage in a structure. Ideally, a damagesensitive feature will change in some consistent manner with increasing damage level. Identifying features that can accurately distinguish a damaged structure from an undamaged one is the focus of most SHM technical literature (Doebling et al., 1996; Sohn et al., 2004). Fundamentally, the feature-extraction process is based on fitting some model, either physicsbased or data-based, to the measured system response data. The parameters of these models or the predictive errors associated with these models then become the damage-sensitive features. An alternate approach is to identify features that directly compare the sensor waveforms or spectra of these waveforms measured before and after damage. Many of the features identified for impedance-based and wave propagation-based SHM studies fall into this category (Kessler et al., 2002; Park et al., 2003; Ihn et al., 2004; Sohn et al., 2004).

The portion of the SHM process that has received the least attention in the technical literature is the development of statistical models to enhance the damage-detection process. Statistical modelling for feature classification is concerned with the implementation of the algorithms that analyze the distributions of the extracted features in an effort to determine the damaged state of the structure. The algorithms used in statistical model development usually fall into three general categories: (i) Group classification; (ii) Regression analysis; and (ii) Outlier detection. The appropriate algorithm to use will depend on the ability to perform supervised or unsupervised learning. Here, supervised learning refers to the case where examples of data from damaged and undamaged structures are available. Unsupervised learning refers to the case where data are only available from the undamaged structure.

Inherent in the data acquisition, feature extraction, and statistical modelling portions of the SHM process are data normalization, cleansing, fusion, and compression. As it applies to SHM, data normalization is the process of separating changes in sensor reading caused by damage from those caused by varying operational and environmental conditions (Farrar, Sohn and Worden, 2001; Sohn et al., 2001; Worden et al., 2002; Sohn et al., 2003; Sohn, 2006). Data cleansing is the process of selectively choosing data to pass on to, or reject from, the feature selection process. Data fusion is the process of combining information from multiple sensors in an effort to enhance the fidelity of the damage detection process. Data compression is the process of reducing the dimensionality of the data, or the features extracted from the data, in an effort to facilitate efficient storage of information and to enhance the statistical quantification of these 
parameters. These four activities can be implemented in either hardware or software and usually a combination of the two approaches is used.

The hierarchical structure of damage detection can be divided into a four-step process, accordingly to Rytter (1993), that answers the following questions (Farrar and Worden, 2007; Worden et al., 2007):

i. Is the damage presented in the system (existence)?

ii. Where is the damage (location)?

iii. What kind of damage is present (type)?

iv. What is the extent of damage (severity)?

The answers to the questions above can be made only in a sequential way, e.g., the answer to the severity of damage can only be made with a priori knowledge of the type of damage. When applied in an unsupervised mode, statistical algorithms are typically used to answer questions regarding the existence and location of damage. When applied in a supervised learning mode and coupled with analytical models, the statistical algorithms can be used to better determine the type of damage and the severity of damage.

\subsection{The Objective of this Report}

Currently, in the SHM field, there are two well-known approaches to separate the changes in the measured system response caused by operational and environmental conditions from those changes caused by damage. The first approach consists of measuring the parameters related to operational and environmental conditions such as temperature, humidity, traffic loads as well as the structural response at different locations. Then, the normal conditions can be parameterized as a function of different operational and environmental conditions. With such a parameterized model, novelty detection processes can be used to detect when the measured system response deviates from the normal condition that corresponds to the appropriate operational and environmental conditions. The second approach, and used in this study, attempts to establish the existence of damage for cases when measures of the operational and environmental parameters that influence the system's dynamic response cannot be measured.

The objective of this study is to apply the LANL statistical pattern recognition paradigm for SHM to data acquired from a laboratory three-story building structure. The report applies various statistical procedures that have been widely used in different engineering fields and briefly reviews the theoretical basis for some of them. More theoretical and detailed developments of the applied statistical procedures are available from the references.

In the hierarchical structure of damage detection, this report addresses the need for robust incipient damage-detection methods. Therefore, it is concerned with determining the existence of damage in the test structure. Even though locating and assessing the severity of damage are important in terms of estimating the residual lifetime of the structures, the reliable detection of damage existence must precede these more detailed damage descriptions. To achieve this goal, this report is mainly focused on feature extraction and statistical modelling for feature classification.

The layout of this report is as follows. Section 2 provides a summary description of the test structure that relates the sources of simulated damage to real-world damage in the structures, the data-acquisition system, and measured data. For the measured data, the undamaged and damaged state conditions as well as the assumptions behind them are defined. In Section 3, the 
physics-based numerical model is summarized. This model was used to validate some assumptions about the system response as well as the measured experimental data. Section 4 provides a general overview on the importance of feature extraction in the SHM process. Moreover, well-established feature extraction procedures are applied to the measured data. This section also points out the main advantages and disadvantages of each of these feature extraction approaches in the current application. The features are extracted in both time and frequency domains in order to explore the different perspectives of the data to the greatest extent. Special attention is given to the autoregressive (AR) model as a feature extraction technique. In Section 5, several statistical procedures for feature classification are presented that discriminate the undamaged and damaged state conditions, with emphasis on the statistical process control (SPC) techniques. In Section 6, the applicability of the autoassociative neural network (AANN), Mahalanobis distance, and singular value decomposition (SVD) to data normalization for feature classification is demonstrated on data measured from the test structure. These algorithms are used to identify signal deviations from the normal condition. Basically, these algorithms classify features from potential damaged conditions as outliers when they are compared to the features extracted from the normal condition.

This report concludes with a general discussion and summary regarding the effectiveness of the various feature extraction and statistical modelling procedures to identify damage in this test structure. 


\section{EXPERIMENTAL PROCEDURE}

\subsection{Test Structure Description}

The three-story building structure shown in Figure 1(a) is used as a damage-detection test-bed structure. The structure consists of aluminum columns and plates assembled using bolted joints, which slides on rails that allow movement in the $\mathrm{x}$-direction only. At each floor, four aluminum columns $(17.7 \times 2.5 \times 0.6 \mathrm{~cm})$ are connected to the top and bottom aluminum plates $(30.5 \times 30.5 \times 2.5 \mathrm{~cm})$ forming a four degree-of-freedom (DOF) system. Additionally, a center column $(15.0 \times 2.5 \times 2.5 \mathrm{~cm})$ is suspended from the top floor. This column is used as a source of damage that induces nonlinear behavior when it contacts a bumper mounted on the next floor, as shown in Figure 1(b). The position of the bumper can be adjusted to vary the extent of impacting that occurs during a particular excitation level.

In the context of SHM, this source of damage is intended to simulate the fatigue cracks that subsequently open and close under operational and environmental loading conditions, or loose connections that rattle. Figure 2 shows a schematic representation along with the basic dimensions of the test structure.

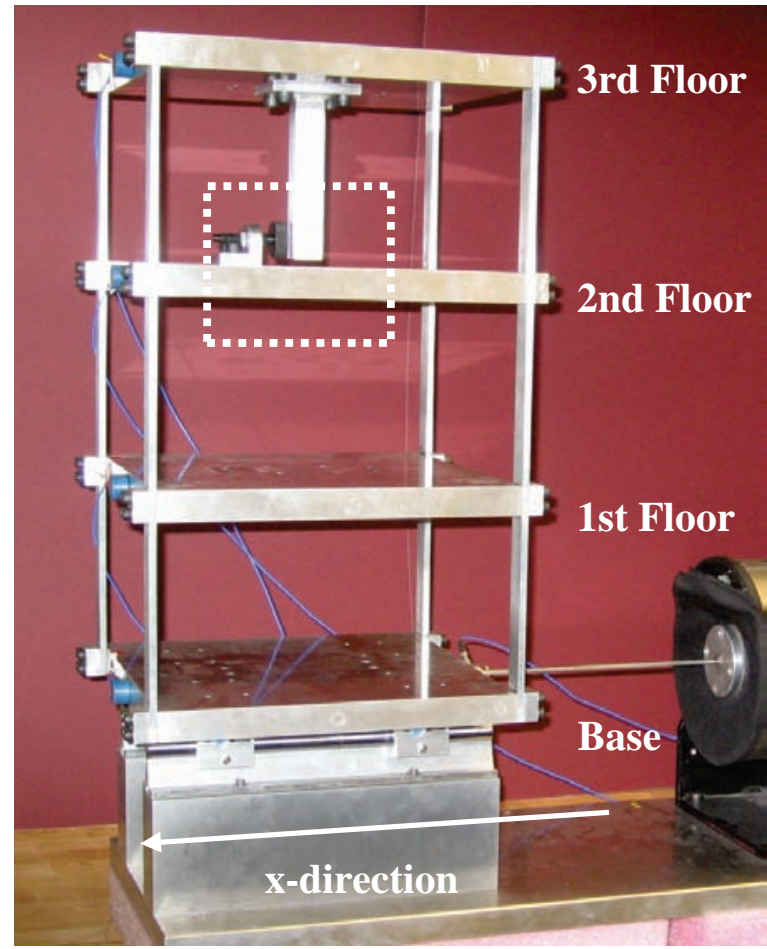

(a) Three-story building structure and shaker

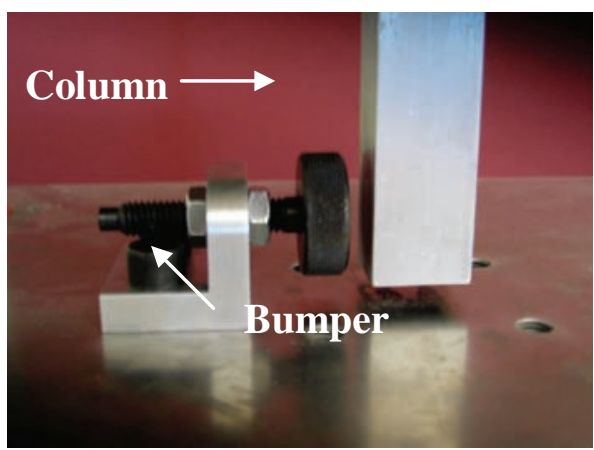

(b) The adjustable bumper and the suspended column

Figure 1: $\quad$ Test structure setup. 

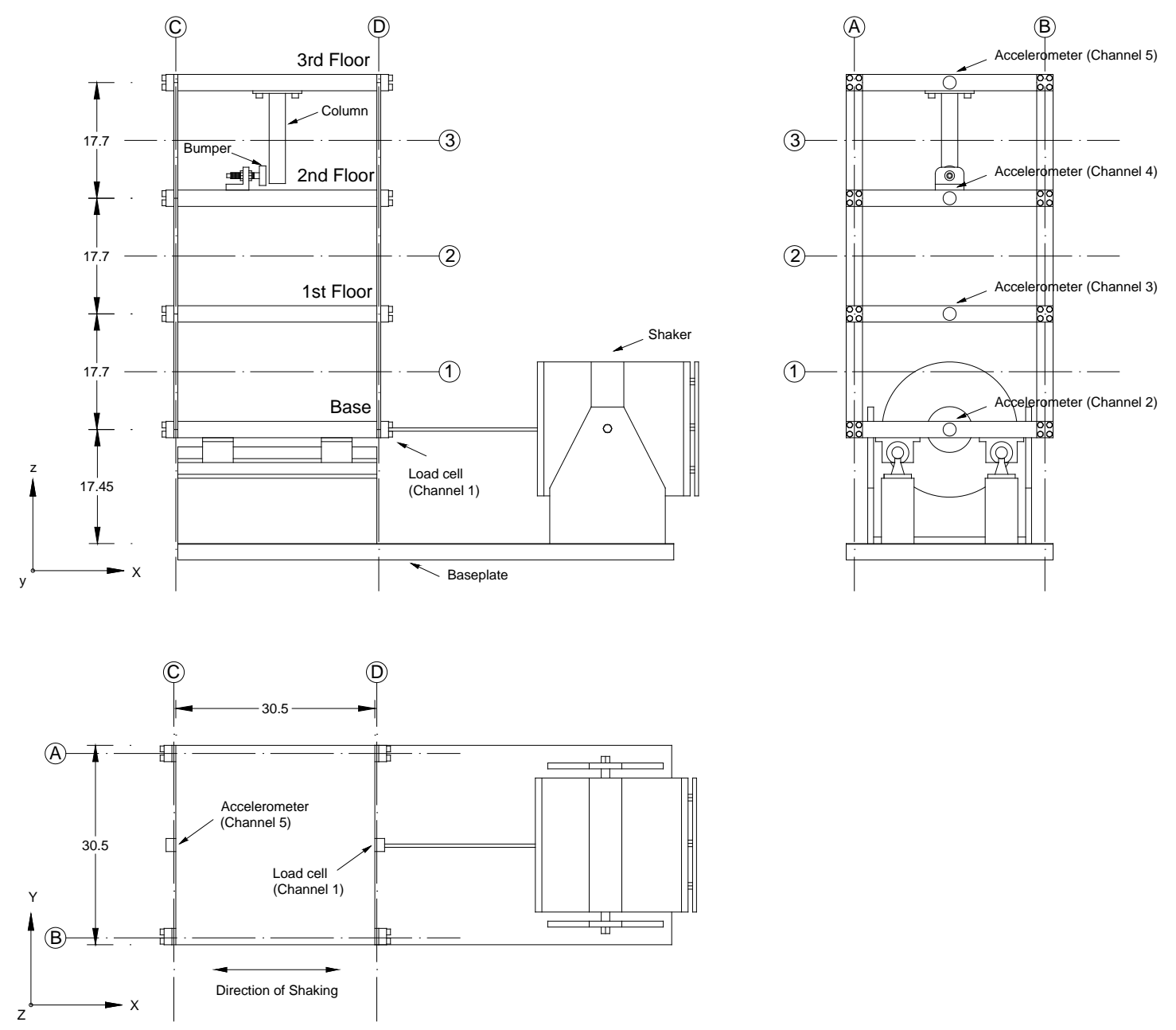

Figure 2: $\quad$ Basic dimensions of the three-story building structure (all dimensions are in $\mathrm{cm}$ ).

\subsection{Data Acquisition System}

An electrodynamic shaker provides a lateral excitation to the base floor along the center line of the structure. The structure and shaker are mounted together on an aluminum baseplate $(76.2 \times 30.5 \times 2.5 \mathrm{~cm})$, and the entire system rests on rigid foam. The foam is intended to minimize extraneous sources of unmeasured excitation from being introduced through the base of the system. A load cell with a nominal sensitivity of $2.2 \mathrm{mV} / \mathrm{N}$ was attached at the end of a stinger to measure the input force from the shaker to the structure. Four accelerometers with nominal sensitivities of $1,000 \mathrm{mV} / \mathrm{g}$ were attached at the center line of each floor on the opposite side from the excitation source to measure the system's response. Because the accelerometers are mounted at the center line of each floor they are insensitive to torsional modes of the structure. In addition, the shaker location and the linear bearings minimize the torsional excitation of the system.

A Dactron Spectrabook data acquisition system was used to collect and process the data. The output channel of this system, which provides the excitation signal to the shaker, is connected to a Techron 5530 Power Supply Amplifier that drives the shaker. The location and characteristics of the five sensor channels (Channels 1-5) used in these tests can be found in Figure 2 and Table 1, respectively. 
Table 1: Characteristics of the Sensors Used Throughout the Testing

\begin{tabular}{|l|l|l|l|}
\hline Channel Number & \multicolumn{1}{|c|}{ Transducer } & \multicolumn{1}{|c|}{ Reference } & Nominal Sensitivity \\
\hline Channel 1 & Load cell & $\begin{array}{l}\text { PCB 208 C03 } \\
\text { SN 22569 }\end{array}$ & $2.2 \mathrm{mV} / \mathrm{N}$ \\
\hline Channel 2 & Accelerometer & $\begin{array}{l}\text { PCB 336C } \\
\text { SN 10099 }\end{array}$ & $1000 \mathrm{mV} / \mathrm{g}$ \\
\hline Channel 3 & Accelerometer & $\begin{array}{l}\text { PCB 336c } \\
\text { SN 10120 }\end{array}$ & $1000 \mathrm{mV} / \mathrm{g}$ \\
\hline Channel 4 & Accelerometer & $\begin{array}{l}\text { PCB 336c } \\
\text { SN 9916 }\end{array}$ & $1000 \mathrm{mV} / \mathrm{g}$ \\
\hline Channel 5 & Accelerometer & $\begin{array}{l}\text { PCB 336C } \\
\text { SN 10100 }\end{array}$ & $1000 \mathrm{mV} / \mathrm{g}$ \\
\hline
\end{tabular}

The analog sensor signals were discretized with 8,192 data points sampled at $3.125 \mathrm{~ms}$ intervals corresponding to a sampling frequency of $320 \mathrm{~Hz}$. These sampling parameters yield time histories of $25.6 \mathrm{~s}$ in duration. When these data were transformed into the frequency domain, the spectra consisted of 3,600 lines displaying the data up to a maximum frequency of $140.6 \mathrm{~Hz}$ at a resolution of $0.0391 \mathrm{~Hz}$. A band-limited random excitation in the range of 20-150 Hz was used to excite the structure. This excitation signal was chosen in order to avoid the rigid body modes of the structure that are present below $20 \mathrm{~Hz}$. The excitation level was set to 2.6 V RMS in the Dactron system, which corresponds to, approximately, $20 \mathrm{~N}$ RMS measured at Channel 1.

\subsection{Measured Data}

Force- and acceleration-time histories (time series or sample records) for a variety of different structural state conditions were collected as shown in Table 2 along with information that describes the different states. For example, the state condition labelled "State \#4" is described as " $87.5 \%$ stiffness reduction in column 1BD," which means there was $87.5 \%$ stiffness reduction in the column located between the base and first floor at the intersection of plane B and $\mathrm{D}$ as defined in Figure 2.

The structural state conditions can be categorized into four main groups. The first group is the baseline condition. The baseline condition is the reference structural state and is labelled State \#1 in Table 2. The bumper and the suspended column are included in the baseline condition, but the spacing between the bumper and the column was maintained in such a way that there were no impacts during the excitation. The second group includes the states when the mass and stiffness of the columns were changed. Real-world structures have operational and environmental variability, which create difficulties in detecting and identifying structural damage. In order to simulate such operational and environmental condition changes, tests were performed with different mass and stiffness conditions (States \#2-\#9). The mass change, $m$, consisted of $1.2 \mathrm{~kg}$ (approximately 19\% of the total mass of each floor) being added to the first floor and to the base, as shown in Figure 3 (a). The stiffness change was introduced by reducing one or more columns' stiffness by $87.5 \%$. This process was done by replacing the corresponded column with another one with half the cross-section thickness in the direction of shaking. The third group includes damaged state conditions simulated through the introduction of nonlinearities into the structure using a bumper and a suspended column, with different gaps between them, as shown in Figure 3 (b). The gap between the bumper and the suspended column 
Table 2: Data Labels of the Structural State Conditions

\begin{tabular}{|c|c|l|}
\hline Label & $\begin{array}{c}\text { State } \\
\text { Condition }\end{array}$ & Description \\
\hline State \#1 & Undamaged & Baseline condition \\
\hline State \#2 & Undamaged & Mass $=1.2 \mathrm{~kg}$ at the base \\
\hline State \#3 & Undamaged & Mass $=1.2 \mathrm{~kg}$ on the $1^{\text {st }}$ floor \\
\hline State \#4 & Undamaged & $87.5 \%$ stiffness reduction in column 1BD \\
\hline State \#5 & Undamaged & $87.5 \%$ stiffness reduction in column $1 \mathrm{AD}$ and $1 \mathrm{BD}$ \\
\hline State \#6 & Undamaged & $87.5 \%$ stiffness reduction in column 2BD \\
\hline State \#7 & Undamaged & $87.5 \%$ stiffness reduction in column $2 \mathrm{AD}$ and $2 \mathrm{BD}$ \\
\hline State \#8 & Undamaged & $87.5 \%$ stiffness reduction in column 3BD \\
\hline State \#9 & Undamaged & $87.5 \%$ stiffness reduction in column 3AD and 3BD \\
\hline State \#10 & Damaged & Gap $=0.20 \mathrm{~mm}$ \\
\hline State \#11 & Damaged & Gap $=0.15 \mathrm{~mm}$ \\
\hline State \#12 & Damaged & Gap $=0.13 \mathrm{~mm}$ \\
\hline State \#13 & Damaged & Gap $=0.10 \mathrm{~mm}$ \\
\hline State \#14 & Damaged & Gap $=0.05 \mathrm{~mm}$ \\
\hline State \#15 & Damaged & Gap $=0.20 \mathrm{~mm}$ and mass $=1.2 \mathrm{~kg}$ at the base \\
\hline State \#16 & Damaged & Gap $=0.20 \mathrm{~mm}$ and mass $=1.2 \mathrm{~kg}$ on the $1^{\text {st }}$ floor \\
\hline State \#17 & Damaged & Gap $=0.10 \mathrm{~mm}$ and mass $=1.2 \mathrm{~kg}$ on the $1^{\text {st }}$ floor \\
\hline
\end{tabular}

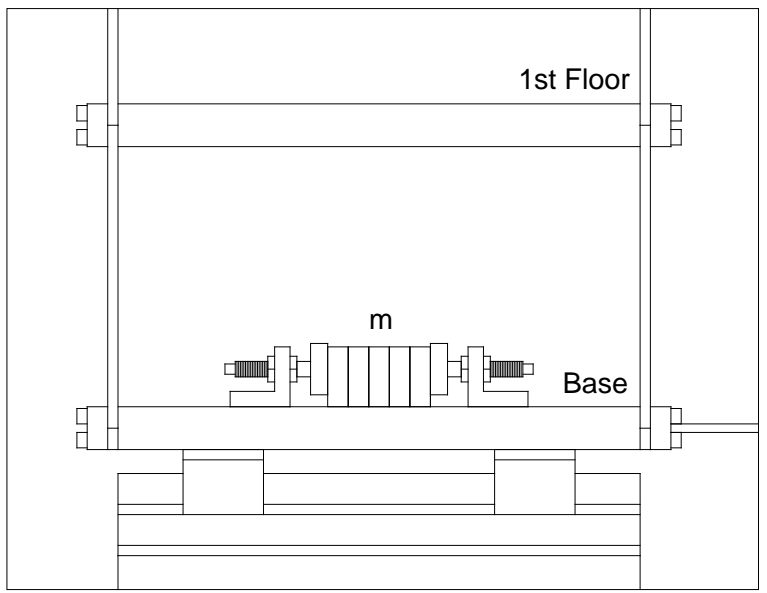

(a) Mass, $m$, added at the base

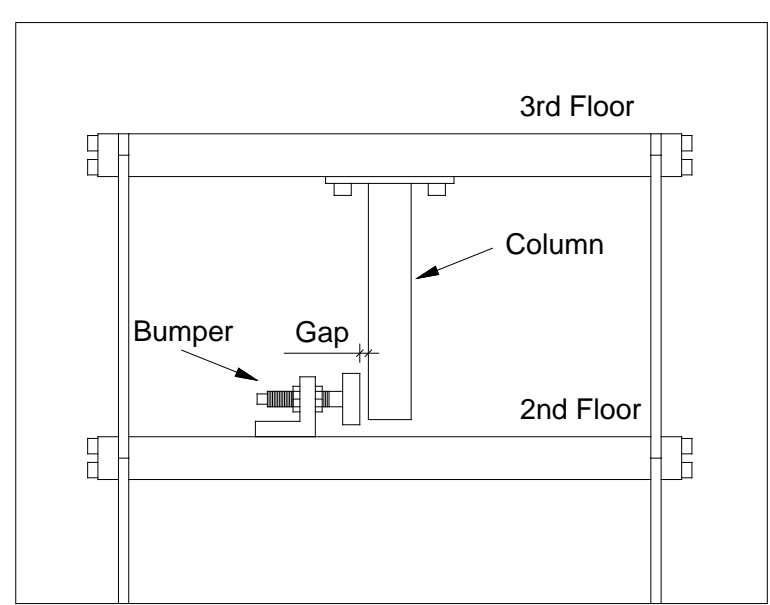

(b) Nonlinearity source

Figure 3: $\quad$ Structural details. 
was varied $(0.20,0.15,0.13,0.10$, and $0.05 \mathrm{~mm})$ in order to introduce different levels of nonlinearity (States \#10-\#14) for a given level of excitation. Finally, the fourth group includes the state conditions with damage in addition to mass and stiffness changes used to model operational and environmental condition changes (States \#15-\#17).

For each state condition, ten tests were performed in order to take into account the variability in the data. Thus, for each of the five transducers, ten time histories were measured in each structural state condition. Additionally, the data acquisition system recorded the associated frequency response functions (FRFs) of the response at Channels 2-5 relative to the input measured with Channel 1 and the corresponding coherence functions. A Hanning window was applied to the time-domain data for leakage reduction and five averages were used to reduce the influence of random noise in the FRF estimates. Note that the Hanning window is commonly used when calculating spectral quantities from random time histories. The recorded time history for each test corresponds to the last time history used in the averaging process.

To illustrate the output results from the data-acquisition system for one test of State \#1, Figure 4 and Figure 5 show the force-time history from Channel 1 and the acceleration-time history from Channel 5, respectively. Figure 6 shows the FRF (y-axis on the left-hand side) relating the input excitation at Channel 1 and response at Channel 5 and its coherence function (y-axis on the right-hand side) calculated from five averages. Notice that the FRF is in logarithmic scale to compress the large signal amplitude and expand the small ones, allowing easier visualization of all frequencies in the signal. The coherence function is a measure of the correlation between the output signal and the input signal at each frequency. If the coherence value is 1 , it suggests that the output is only caused by the input. If the coherence value is 0 , then it suggests that none of the output is linearly correlated with the input. In Figure 6 the coherence function is, in general, close to 1 , giving an indication that the responses are directly correlated with the input.

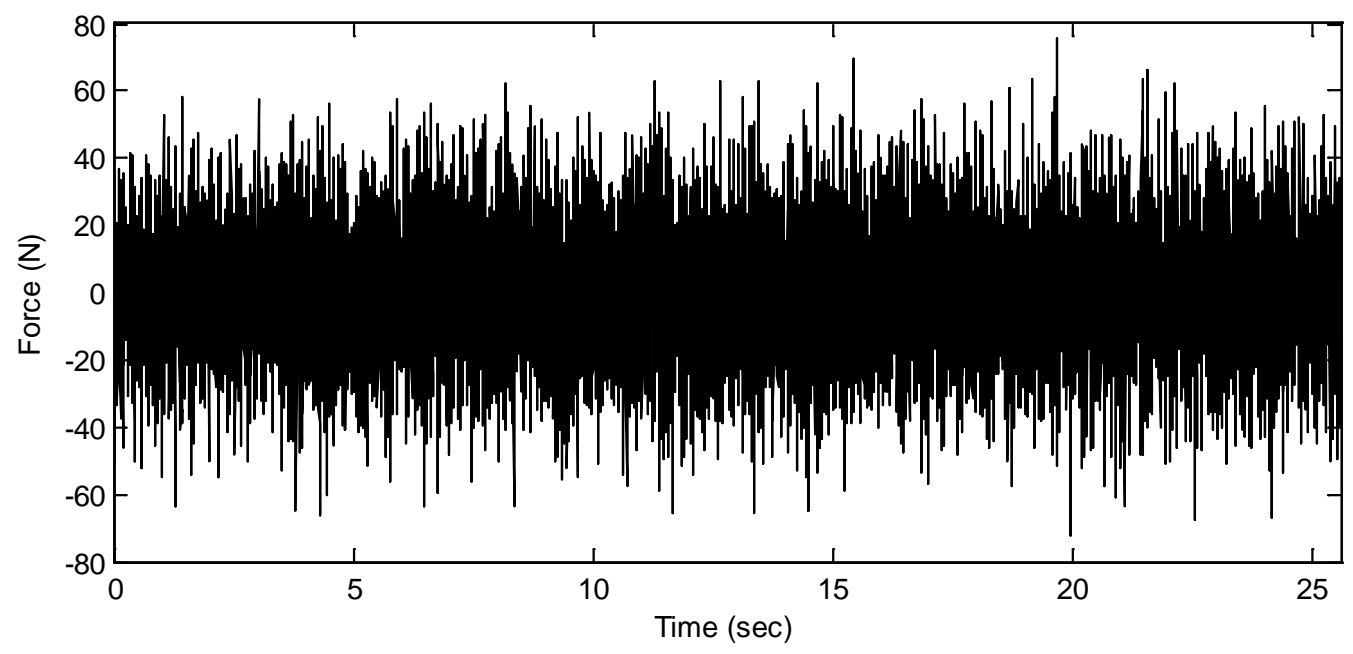

Figure 4: $\quad$ Force-time history from Channel 1 of State \#1. 


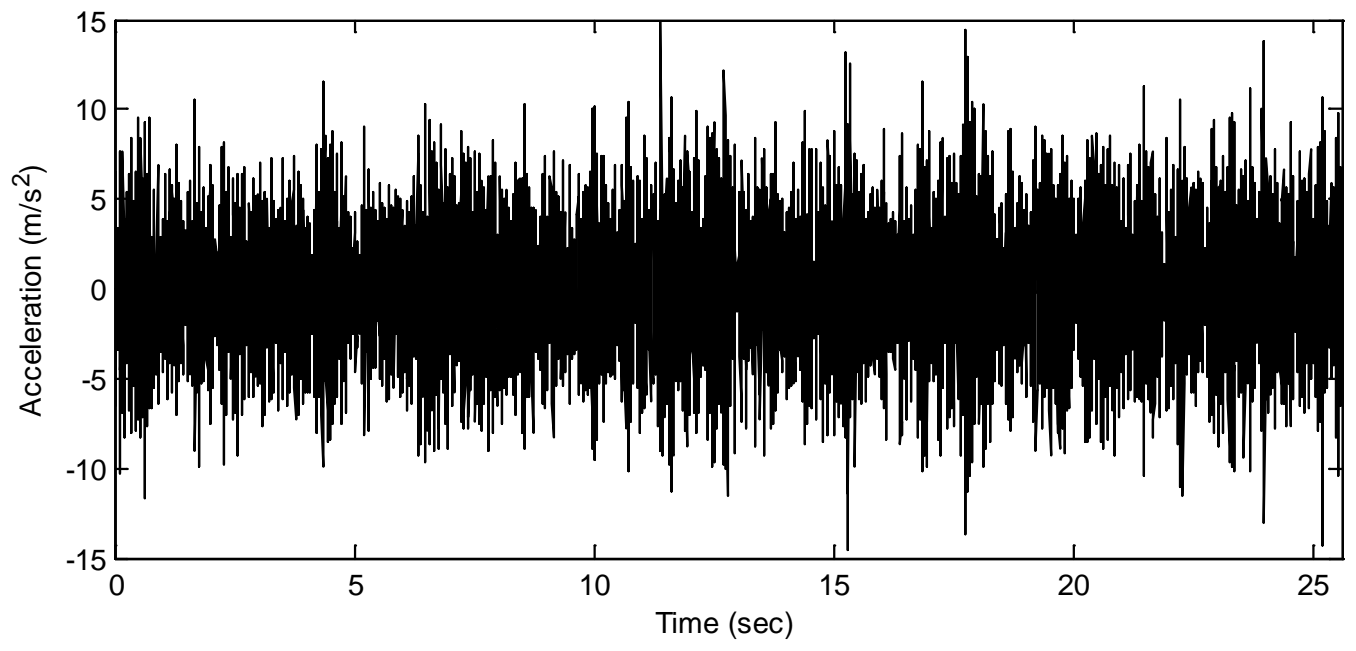

Figure 5: $\quad$ Acceleration-time history from Channel 5 of State \#1.

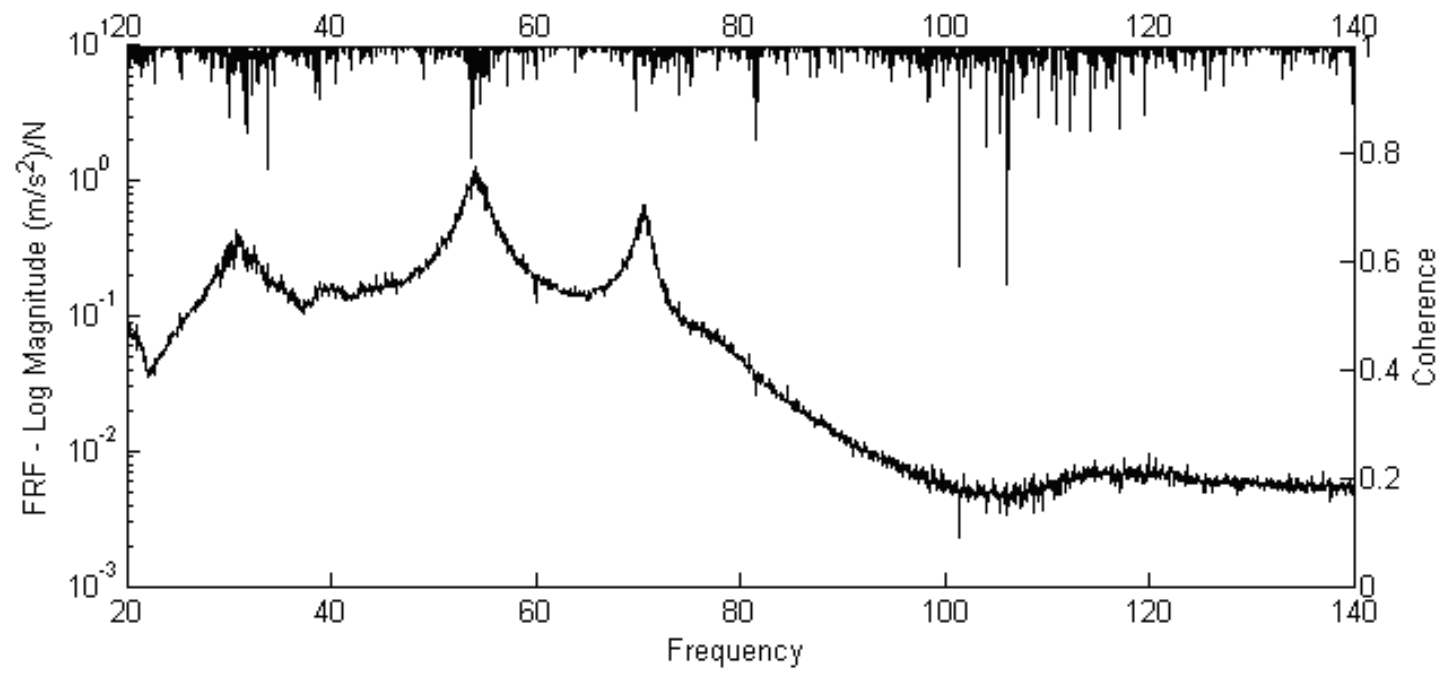

Figure 6: $\quad$ FRF and coherence function from Channel 5 of State \#1.

\subsubsection{Operational and Environmental Effects}

Many papers and reports discuss the changes in structural characteristics caused by operational and environmental condition changes (Woon and Mitchell, 1996; Soon et al., 1999). The most pronounced structural changes have been associated with temperature variations. For example, the measured fundamental frequency of the Alamosa Canyon Bridge in New Mexico varied approximately 5\% during a 24-hour test period, and this variability was correlated to the temperature difference across the bridge deck (Sohn et al., 1999). The physical reason for this correlation is the temperature dependence of the material properties, such as Young's modulus, Poisson's ratio, coefficient of thermal expansion (which can result in a change in the boundary conditions), and damping. Such variability often manifests itself in linear changes in the stiffness or mass of a structure. For a given structure, if the percent shift in natural frequency, relative to the reference temperature, is approximately the same for each mode (Woon and Mitchell, 1996), 
it implies that temperature change affects the elastic modulus. Moreover, changes in the natural frequencies can also be correlated to changes in mass. In the case of bridges, a significant volume of traffic increases the mass of the structure and consequently decreases the natural frequencies.

These factors have posed challenges when using modal properties as damage-sensitive features. As stated above, in order to simulate the changes in real-world structures caused by varying operational and environmental conditions, several sources of variability were included in the test structure. This variability includes adding mass and reducing the stiffness at several different locations. Figure 7 shows the FRFs, of the response from Channel 5 and the excitation force at Channel 1, for the baseline condition state (State \#1) and an undamaged state with operational variations corresponding to a 1.2-kg mass attached on the first floor (State \#3). As expected, the case with the added mass resulted in lowering the natural frequencies.

\subsubsection{Nonlinearities Effects}

The simulated damage was introduced through nonlinearities resulting from impacts with a bumper, as shown in Figure 3(b). When the structure is excited at the base, the suspended column hits the bumper. The level of nonlinearity depends on the amplitude of oscillation and the gap between the column and the bumper. As described above, this source of damage is intended to simulate fatigue cracks that open and close upon dynamic loading. Figure 8 shows FRFs of the response from Channel 5 and the excitation force at Channel 1 for the baseline condition state (State \#1), the damaged state consisting of a gap of $0.05 \mathrm{~mm}$ (State \#14), and for the damaged state consisting of a gap of $0.10 \mathrm{~mm}$ and $1.2 \mathrm{~kg}$ added on the first floor (State \#17). The figure shows shifts of the resonance frequencies as well as distortions of the FRF shape caused by the nonlinearities.

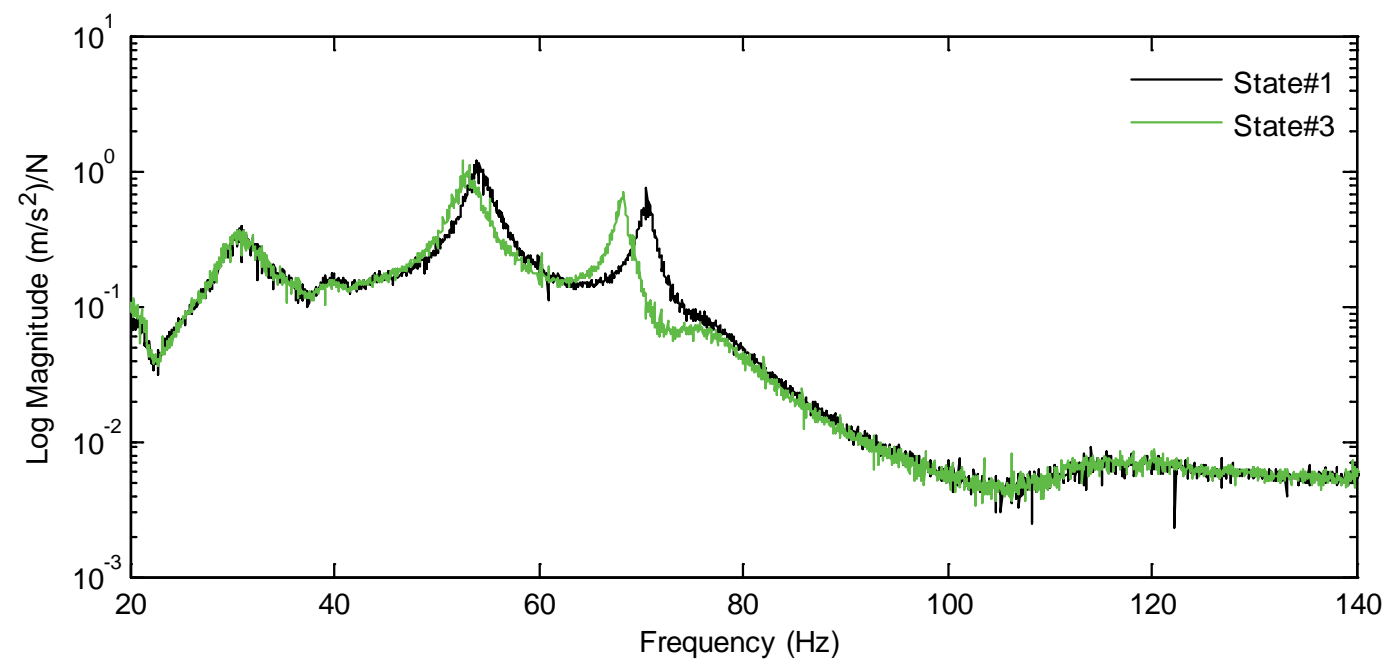

Figure 7: $\quad$ FRFs (based on five averages) of the response at Channel 5 and the excitation force at Channel 1 for State \#1 (baseline) and State \#3 (1.2-kg added mass on the first floor). 


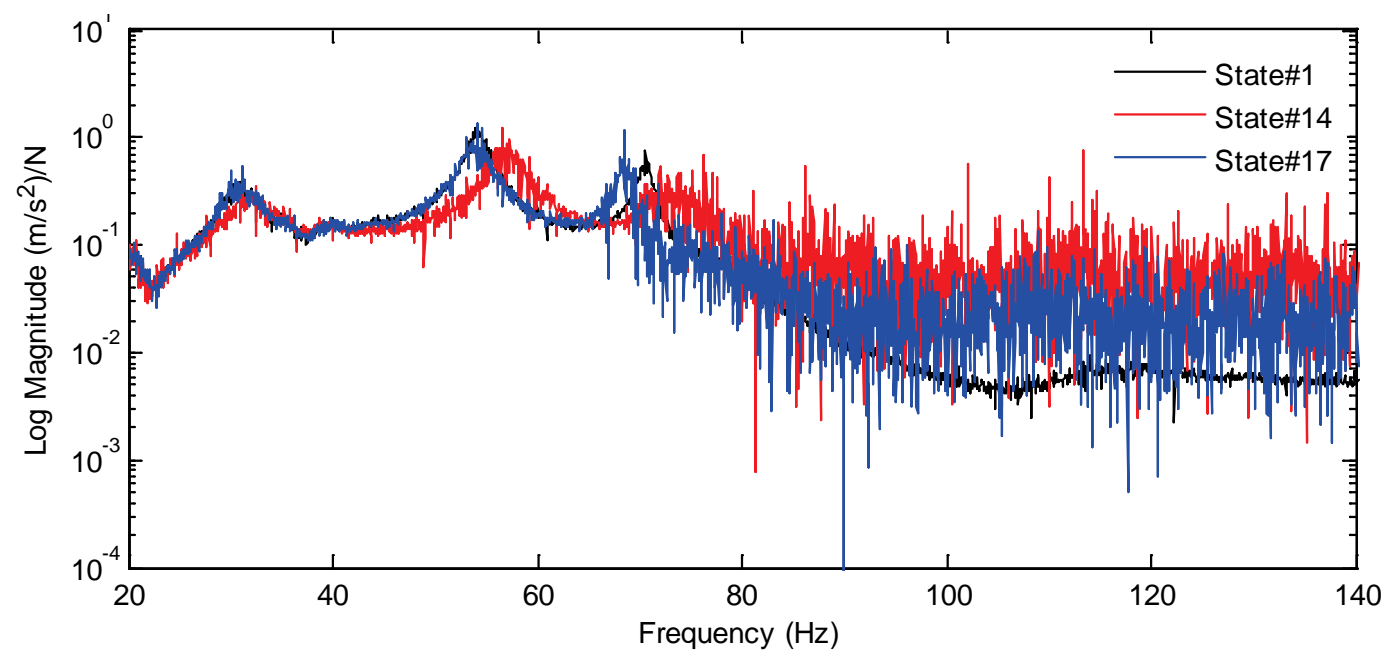

Figure 8: $\quad$ FRFs (based on five averages) of the response at Channel 5 and the excitation force at Channel 1 for State \#1 (baseline), State \#14 (gap = $0.05 \mathrm{~mm}$ ), and State \#17 (gap $=0.10 \mathrm{~mm}$ and 1.2-kg added mass on the first floor). 


\section{NUMERICAL SIMULATION}

The goal of this section is to develop a physics-based numerical model of the test structure in order to compare the numerical results with the measured experimental data. In general, numerical models can be used to define the SHM system properties, such as sensor type and location, before deploying a monitoring system on real-world structures and to validate some SHM systems' measurements.

The test structure is modeled as four lumped masses at the floors, including the base that slides on rails, as shown in Figure 9. The stiffness $k_{1}$ and damping $c_{1}$ are intended to simulate the friction between the rails and the structure.

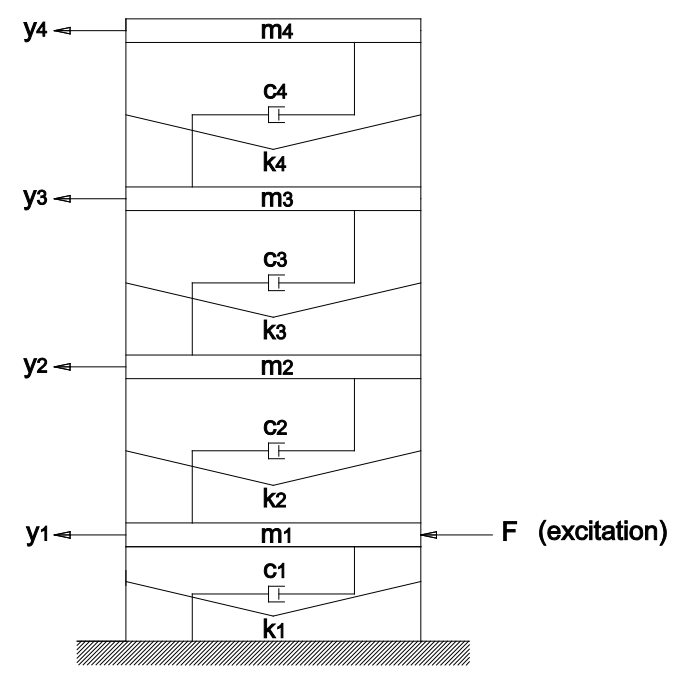

Figure 9: $\quad$ Shear-building model of the test structure.

For the shear-building model shown in Figure 9, the equations of motion can be written in a matrix notation as follows:

$$
[M]\{\ddot{y}\}+[C]\{\dot{y}\}+[K]\{y\}=\{F(t)\},
$$

where the $[M],[C]$, and $[K]$ are the mass, damping, and stiffness matrices of the system, respectively, $\{F(t)\}$ is the input vector excitation, and $\{y\}$ is the vector of unknown displacements at the nodal coordinates. The mass and stiffness matrices are given by

$$
[M]=\left[\begin{array}{cccc}
m_{1} & 0 & 0 & 0 \\
0 & m_{2} & 0 & 0 \\
0 & 0 & m_{3} & 0 \\
0 & 0 & 0 & m_{4}
\end{array}\right] \quad[K]=\left[\begin{array}{cccc}
k_{1}+k_{2} & -k_{2} & 0 & 0 \\
-k_{2} & k_{2}+k_{3} & -k_{3} & 0 \\
0 & -k_{3} & k_{3}+k_{4} & -k_{4} \\
0 & 0 & -k_{4} & k_{4}
\end{array}\right] \text {, }
$$

where for $i=1, \ldots, 4, m_{i}$ denotes the mass at the $i^{\text {th }}$ floor and $k_{i}$ denotes the stiffness of the $i^{\text {th }}$ story. The equations of motion can be uncoupled with the modal damping assumption (Naeim and Kelly, 1999), in which $[C]$ can be evaluated from the following modal damping matrix 


$$
\left[C_{n}\right]=\left[\begin{array}{cccc}
2 \zeta_{1} \omega_{1} M_{1} & 0 & 0 & 0 \\
0 & 2 \zeta_{2} \omega_{2} M_{2} & 0 & 0 \\
0 & 0 & 2 \zeta_{3} \omega_{3} M_{3} & 0 \\
0 & 0 & 0 & 2 \zeta_{4} \omega_{4} M_{4}
\end{array}\right] \text {, }
$$

where $\zeta_{i}, \omega_{i}$, and $M_{i}$ are the damping ratio, natural frequency and modal mass, respectively, associated with the $i^{\text {th }}$ mode. The modal damping ratios are estimated from the measured experimental data. The matrices $[C]$ and $\left[C_{n}\right]$ can be related by

$$
\left[C_{n}\right]=[\phi]^{T}[C][\phi],
$$

where $[\phi]$ is the mode shapes' matrix. As a consequence, the matrix $[C]$ is determined by

$$
[C]=[\phi]^{-T}\left[C_{n}\right][\phi]^{-1} .
$$

For the numerical model described above, Figure 10 shows the comparison between the numerical and experimental mode shapes. Notice that the first mode is a rigid body mode and, for convenience, is not illustrated here (see Section 2). The experimental modal parameters were estimated using the rational-fraction polynomial (RFP) method as described later in Subsection 4.3.1. The experimental natural frequencies and damping ratios for the baseline condition (State \#1) as well as the numerical natural frequencies are summarized in Table 3. Note that the numerical model assumes, for the aluminium, a calibrated Young's Modulus equal to $65 \mathrm{GPa}$ and negligible friction between the rails and the structure.

One common technique of comparing numerical and experimental mode shapes is by means of modal assurance criterion (MAC) (Maia and Silva, 1997), which is defined as follows:

$$
\operatorname{MAC}\left(\left\{\phi_{N}\right\}_{i},\left\{\phi_{E}\right\}_{j}\right)=\frac{\left(\left\{\phi_{N}\right\}_{i}^{T}\left\{\phi_{E}\right\}_{j}\right)^{2}}{\left(\left\{\phi_{N}\right\}_{i}^{T}\left\{\phi_{N}\right\}_{i}\right)\left(\left\{\phi_{E}\right\}_{j}^{T}\left\{\phi_{E}\right\}_{j}\right)} .
$$

The subscripts $N$ and $E$ refer to numerical and experimental, respectively, and $i$ and $j$ refer to the particular modes that are being compared. The MAC value is bounded between 0 and 1 with a MAC value close to 1 indicating close correlation. As shown in the Figure 11, the MAC values of the second, third, and fourth mode shapes are very close to one, giving an indication that the numerical and experimental mode shapes are highly correlated.

Figures 12 (a) and (b) show the experimental and the numerical acceleration responses at the third floor (Channel 5), respectively. The experimental response corresponds to the measured time-history response of the baseline condition (State \#1) resulting from the excitation measured at Channel 1. The numerical response corresponds to the acceleration at the same location calculated for the same experimental excitation. Apparently, the figure indicates that the acceleration amplitudes of both responses are consistent. Two techniques can be used to numerically compare both responses, namely, the correlation analysis and power spectral density (PSD) comparison. 
(a) 2nd mode

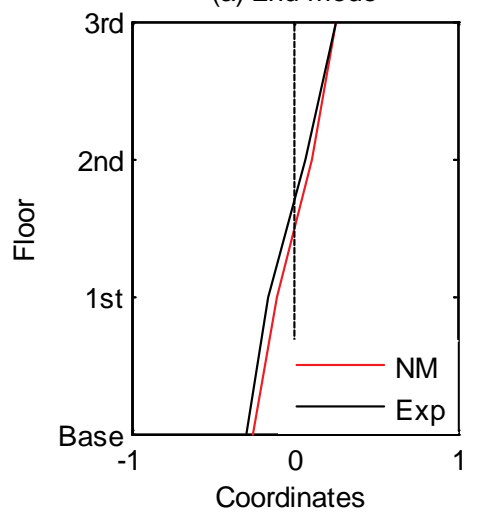

(b) 3rd mode

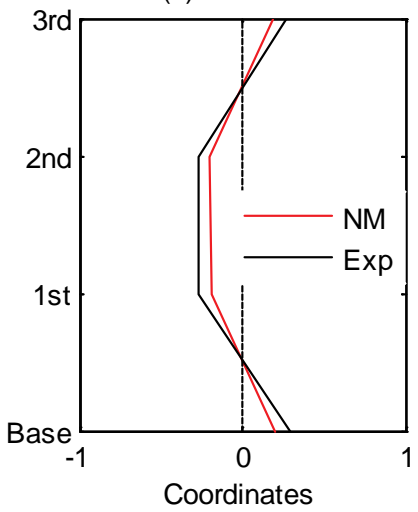

(c) 4th mode

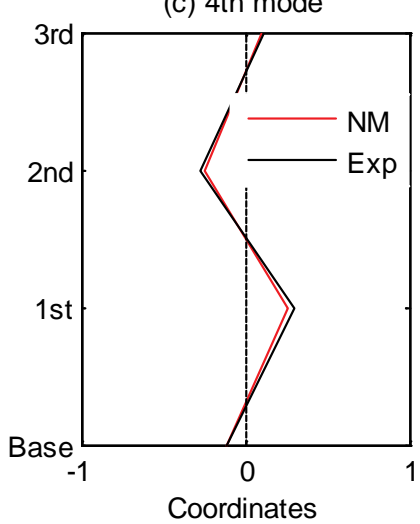

Figure 10: Numerical (NM) and experimental (Exp) mode shapes of the baseline condition.

Table 3: Experimental and Numerical Modal Parameters

\begin{tabular}{|c|c|c|c|}
\hline \multirow{2}{*}{$\begin{array}{c}\text { Mode } \\
\text { number }\end{array}$} & \multicolumn{2}{|c|}{ Frequency (Hz) } & \multirow{2}{*}{$\begin{array}{c}\text { Damping } \\
\text { ratio (\%) }\end{array}$} \\
\cline { 2 - 3 } & Experimental & Numerical & 6.3 \\
\hline 2 & 30.7 & $29.8(-2.9 \%)$ & 2.0 \\
\hline 3 & 54.2 & $54.0(-0.4 \%)$ & 0.97 \\
\hline 4 & 70.7 & $71.6(+1.3 \%)$ & \\
\hline
\end{tabular}

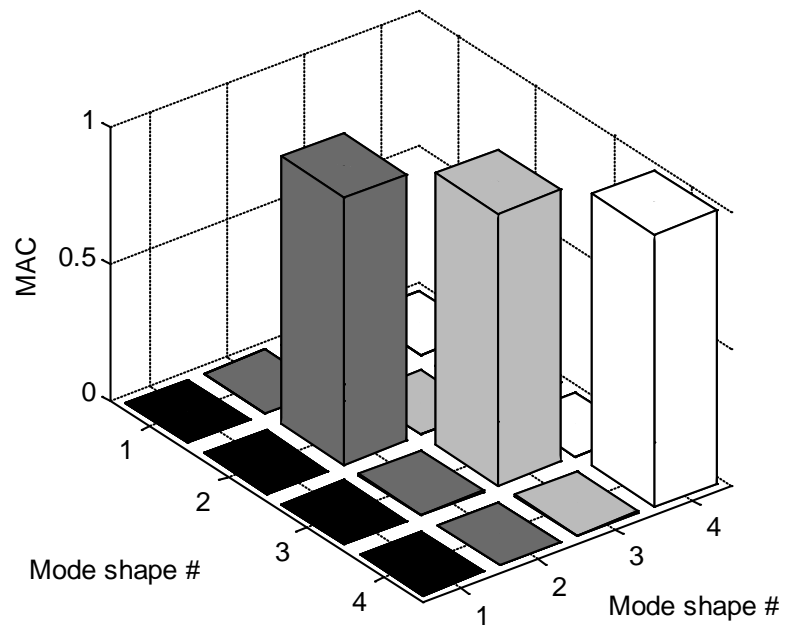

Figure 11: Correlation between numerical and experimental mode shapes by MAC. 
(a) Experimental time history

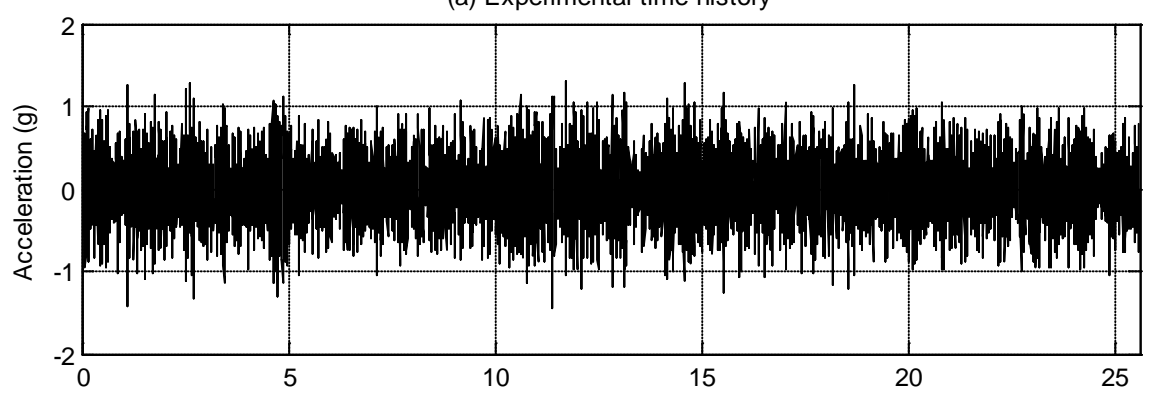

(b) 4-dof numerical model

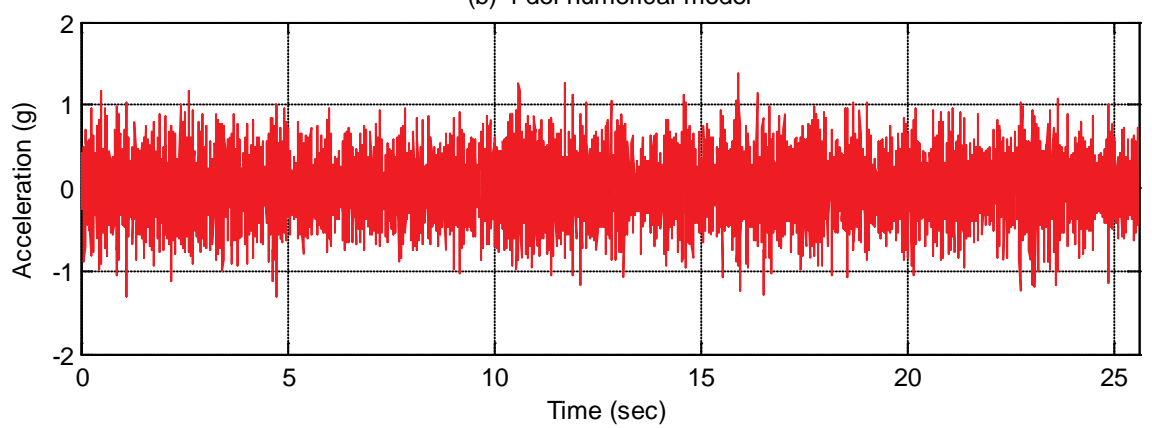

Figure 12: Experimental and numerical responses of the baseline condition from Channel 5, resulting from the measured experimental excitation at Channel 1.

Correlation is a statistical technique used in signal processing for analyzing the relationship between two or more signals. The cross-correlation is a standard technique of estimating the degree to which two different signals are correlated. Considering two random variables $X$ and $Y$, the cross-correlation $R_{X Y}$ at delay or time lag $\tau$ is defined as

$$
R_{X Y}(\tau)=\frac{E\left[\left(X_{t}-\mu_{X}\right)\left(Y_{t+\tau}-\mu_{Y}\right)\right]}{\sigma_{X} \sigma_{Y}},
$$

where $\mu_{X}$ and $\mu_{Y}$ are the means of the corresponding variables, $E$ is the expected value, and $t$ is an integer for a discrete-time process. The standard deviations of the variables, $\sigma_{X}$ and $\sigma_{Y}$, in the denominator, serve to normalize the range of the correlation, and for a stationary process, its values are in the range $[-1,1]$. A coefficient equal to 1 indicates perfect correlation, i.e., the variables overlap when they are shifted by $\tau$, and -1 indicates perfect anticorrelation where the variables are completely out of phase when shifted by $\tau$.

Autocorrelation function is the correlation of a signal with itself, and for the variable $X$ is defined as

$$
R_{X X}(\tau)=\frac{E\left[\left(X_{t}-\mu_{X}\right)\left(X_{t+\tau}-\mu_{X}\right)\right]}{\sigma_{X}} .
$$

The autocorrelation function is used in order to find out repeating patterns within the responses, such as the presence of periodic signals buried under noise. As a general rule, it is sufficient to compute the values of the autocorrelation for values of $\tau \leq n / 4$, where $n$ is the number of data points in the signal (Montgomery, 1997). In this case, by overlapping the numerical and experimental autocorrelation function, this procedure permits us to evaluate if the numerical response reasonably characterizes the experimental data. The autocorrelation functions 
shown in Figure 13, in an overlap format, indicate that the experimental and numerical responses are correlated.

Another manner to compare the accuracy of the experimental and numerical responses is by means of overlapping the PSDs of both responses, as shown in Figure 14. In this case, each PSD is estimated by averaging small spectrum quantities of segments from the time history. Basically, for each response, the associated time history (8,192-point length) is divided into segments according to a moving window of 1,024-point length. Each segment is windowed with a Hamming window. The fast Fourier transform is computed in each segment using a moving window with $50 \%$ overlap. Finally, the set of spectrum estimates is averaged to form the final PSD. In the figure, the numerical estimated PSD seems to fit the experimental PSD, even though the former has more energy content in the second and less energy in the third natural frequencies.

In conclusion, the results of this section summarize the development of a 4-DOF physicsbased numerical model to better understand the structural behavior of the system. The damping matrix for this model was obtained using the results of the experimental modal analysis. The Young's modulus was adjusted so that the analytically predicted natural frequencies agreed with the experimental ones. The developed numerical model assumes negligible friction between the rails and the structure. The model shown predicts the measured baseline response with a high level of certainty.

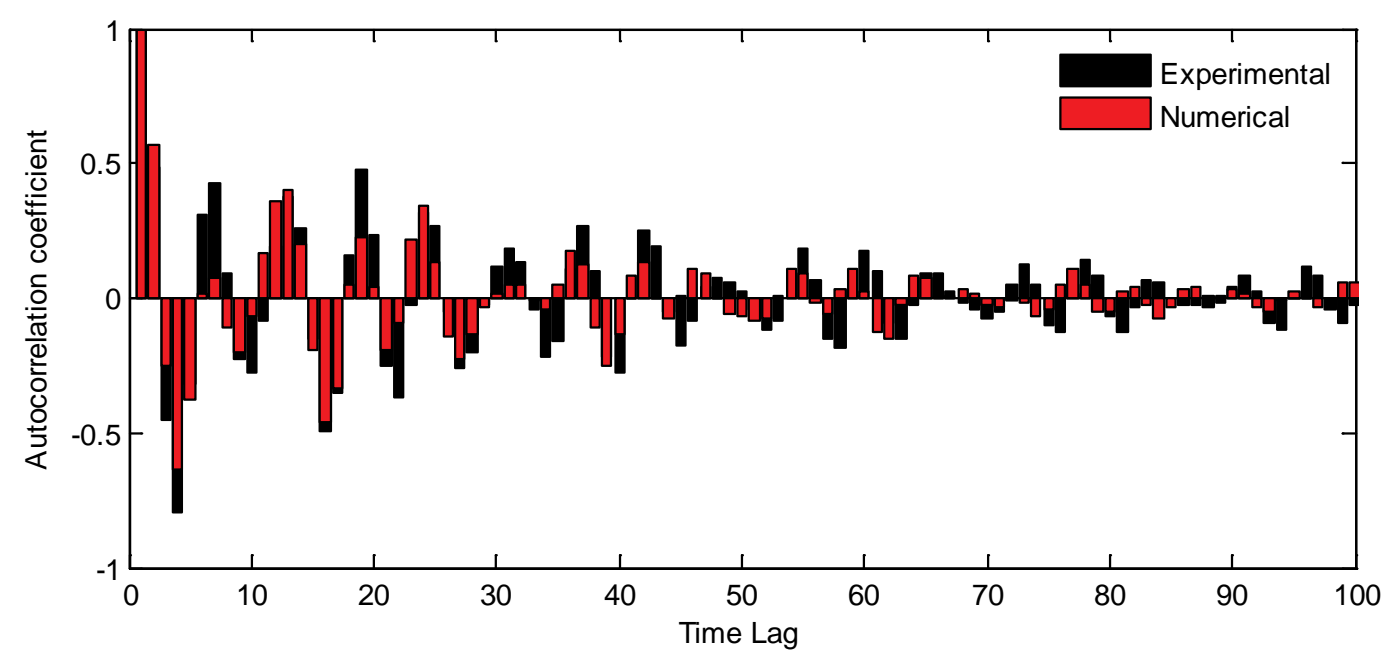

Figure 13: Autocorrelation functions of the experimental and numerical responses. 


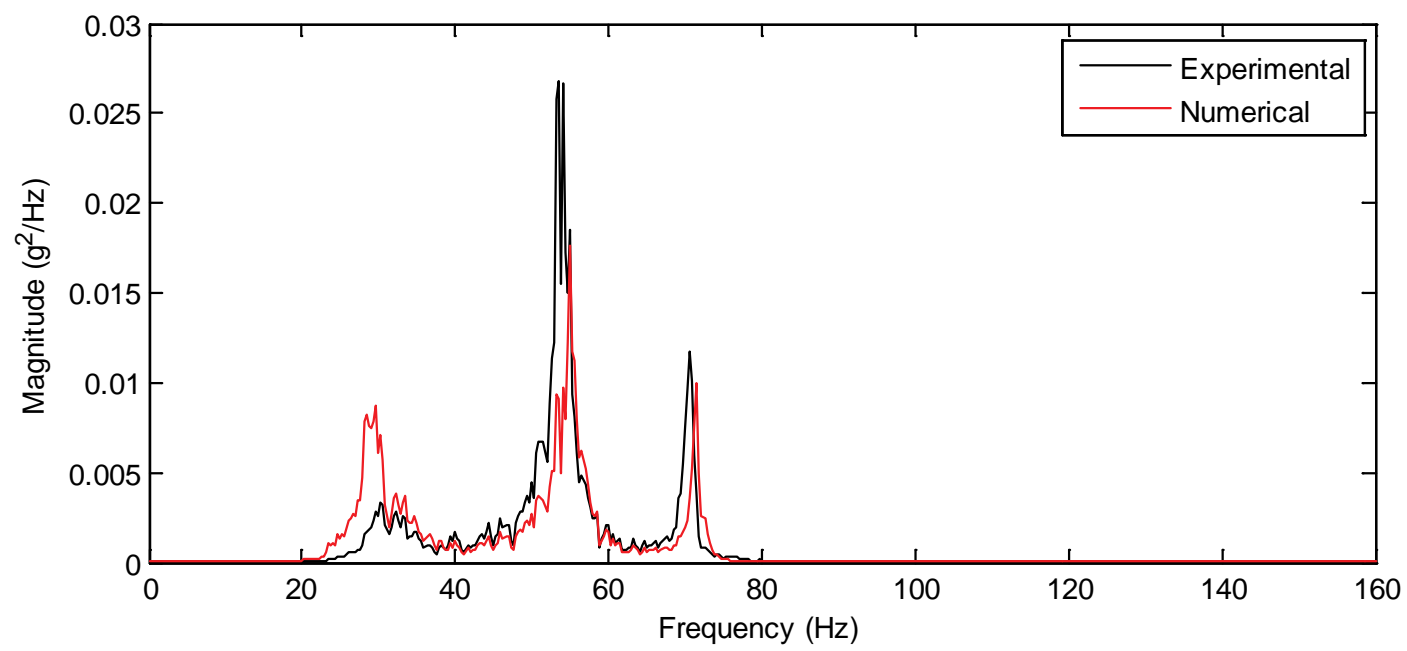

Figure 14: PSD of the experimental and numerical time histories. 


\section{FEATURES EXTRACTION}

In the SHM field, the ideal approach for features selection is to choose features that are sensitive to damage, but are not sensitive to operational and environmental variations. However, in real-world structures such an approach is not always possible, and intelligent feature extraction procedures are usually required (Worden et al., 2007). In this section, several feature extraction procedures are presented, based on basic-statistics analysis, time-series analysis, frequency-domain analysis, and time-frequency analysis.

Because no explicit mathematical equation can be written for the time histories produced by a random phenomenon, such as the measured data in this report, statistical procedures must be used to define the properties of the data. In statistics, the data from a random process is said to be stationary and ergodic when the moments and joint moments are time invariant and they do not change when computed over different time histories (Bendat et al., 2000). In this report some of the basic statistical properties such as the first four statistical moments as well as autocorrelation functions are used to describe the random data. A more detailed theoretical explanation about these basic statistics will be given later, namely in Subsection 4.1. First, a global view of the first four statistical moments for all ten time histories from Channel 5 of each state condition is presented from Figure 15 to Figure 18.

The randomness highlighted in Figure 15 is related to the plot's scale, and it turns out to be meaningless because the mean is approximately zero for all state conditions. The standard deviation (Figure 16), skewness (Figure 17), and kurtosis (Figure 18) do not vary significantly when computed for each of the ten time histories associated with a particular damaged condition. Furthermore, the autocorrelation functions shown in Figure 19 for State \#1 (baseline) and State \#14 (highest level of damage) are relatively consistent for all ten time histories corresponding to the respective condition.

Based on the previous observations, it is assumed that the random processes in this study are weakly stationary or stationary in the wide sense. This assumption implies that the statistics of a single time history are representative of the entire time-history ensemble corresponding to a specific damaged state.

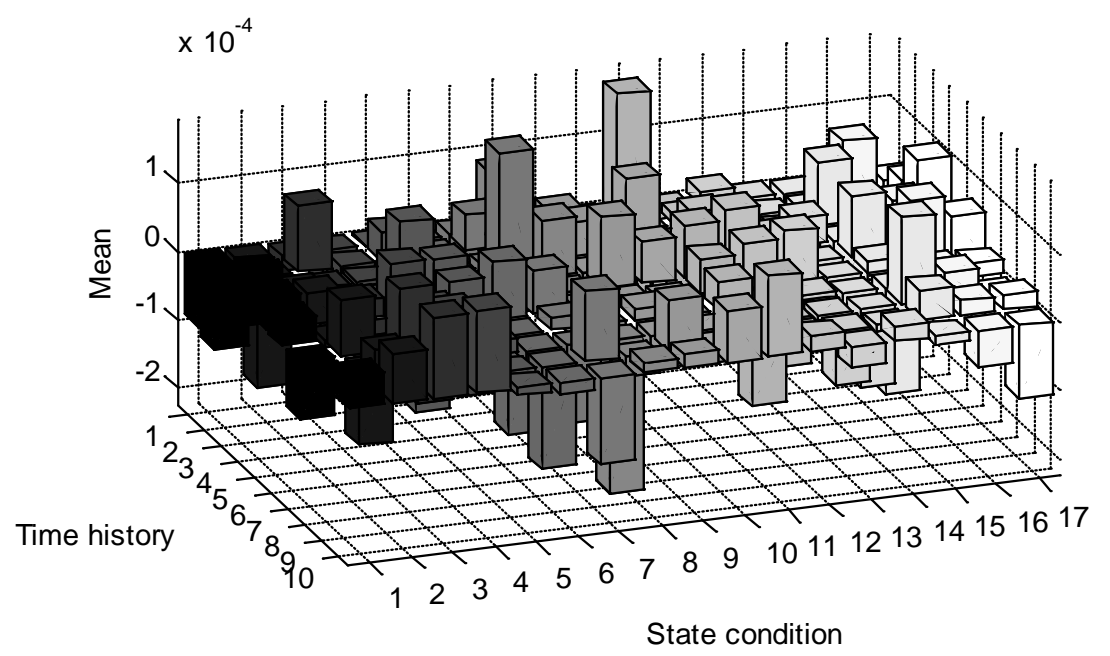

Figure 15: $\quad$ Mean for all time histories from Channel 5 of all state conditions. 


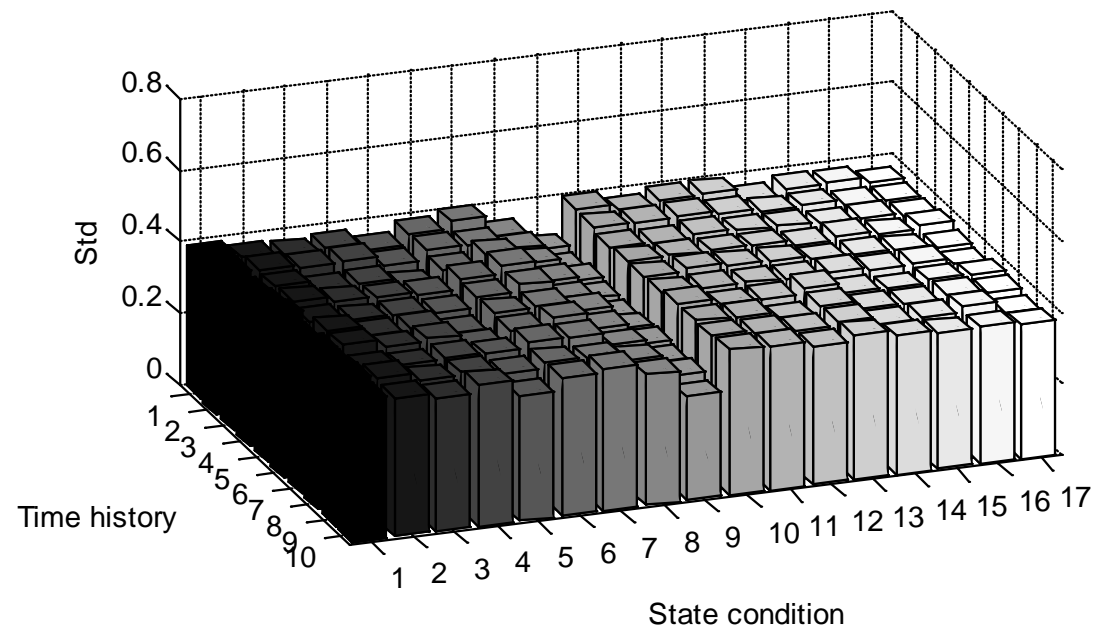

Figure 16: Standard deviation for all time histories from Channel 5 of all state conditions.

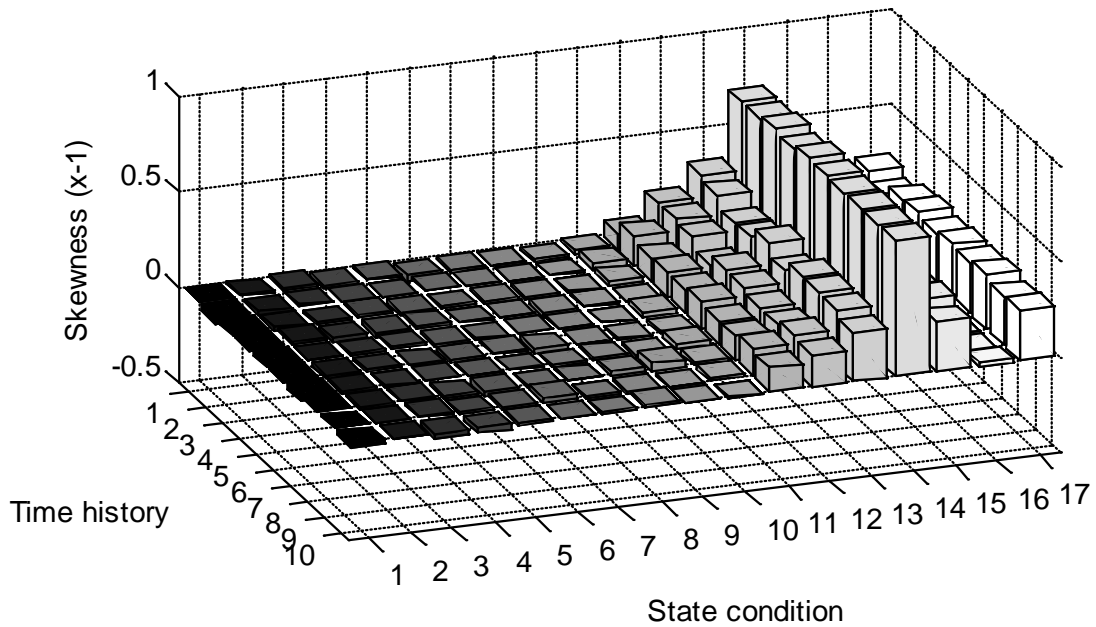

Figure 17: Skewness for all time histories from Channel 5 of all state conditions.

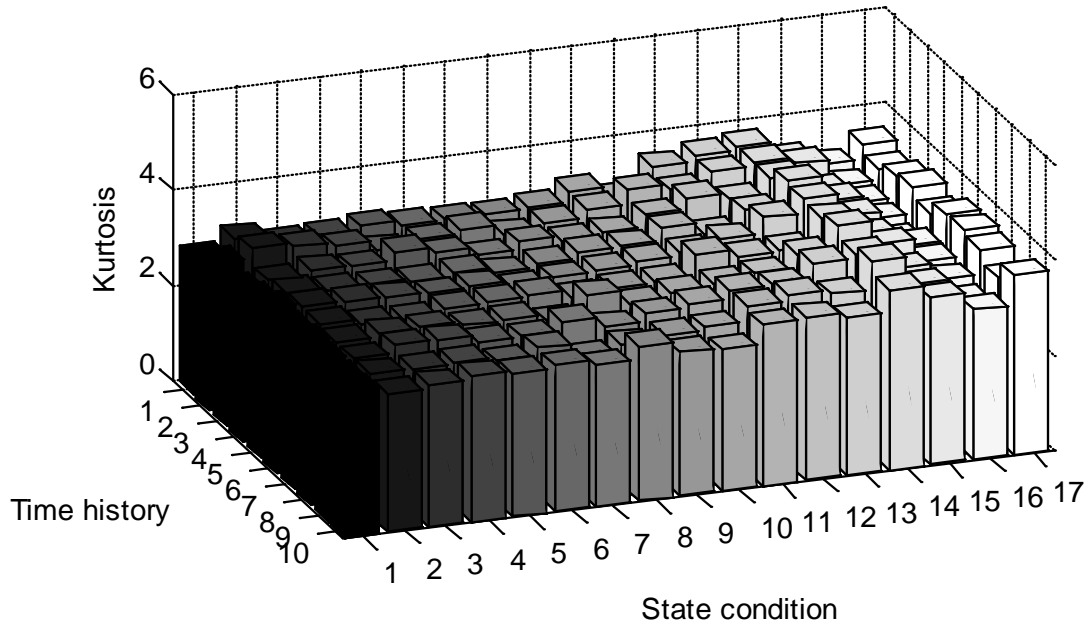

Figure18: Kurtosis for all time histories from Channel 5 of all state conditions. 
(a) State\#1

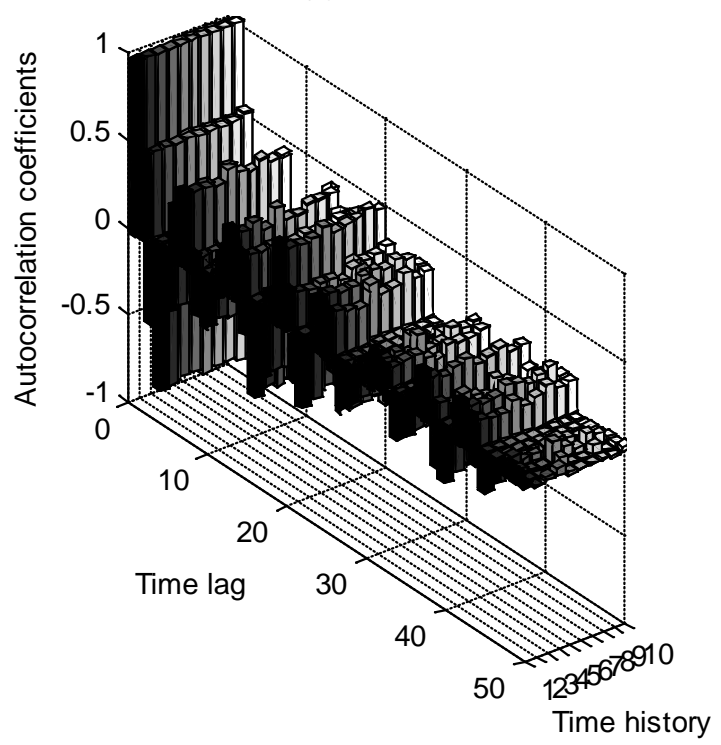

(b) State\#14

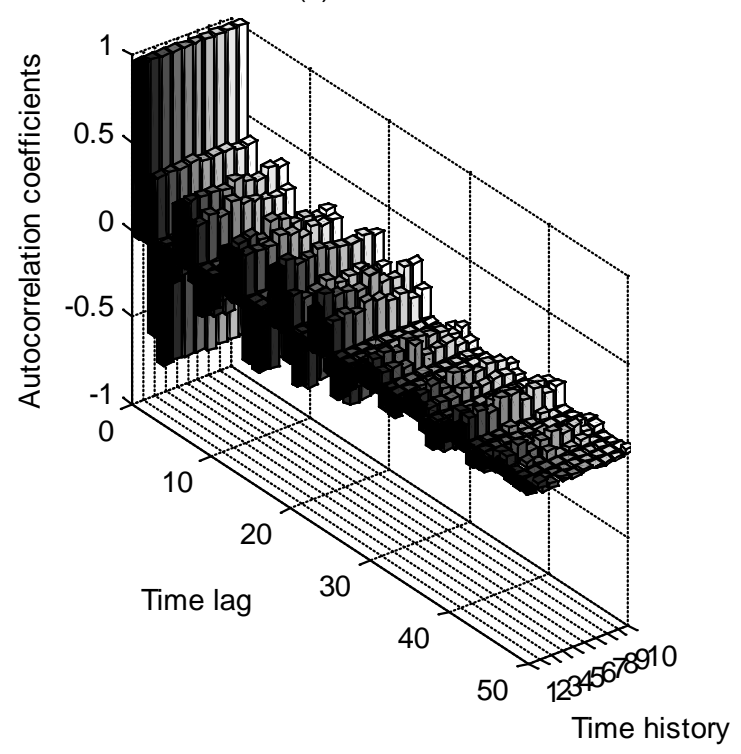

Figure 19: Autocorrelation functions for all time histories from Channel 5 of State \#1 and State \#14.

\subsection{Basic Statistics}

This subsection is concerned with the extraction of basic signal statistics as damagesensitive features. In order to show an overview of the raw data's appearance, acceleration-time histories of States \#1, \#3, \#7, \#14, and \#17 from Channels 2 to 5 are plotted, in concatenated form, in Figure 20. From these plots it can be seen that the amplitude of the time histories is relatively consistent, based on visual inspection. Because the input to the shaker was specified as a normal distribution random signal, it is assumed that the time histories from the baseline and other undamaged conditions are also normally distributed. This assumption is based on the wellknown result from random vibrations (Wirsching et al., 1995) that a linear system subjected to a normally distributed random input will exhibit a normally distributed random response.

In order to establish the underlying distribution of the data, statistical techniques are used to estimate the probability density function (PDF) of the measured data. Moreover, it is expected that the damage can introduce significant changes in the acceleration-time-history PDFs and, as a consequence, the first four statistical moments and PDFs are discussed as damage-sensitive features.

\subsubsection{First Four Statistical Moments of the Measured Data}

The first four statistical moments (mean, standard deviation, skewness, and kurtosis) are often computed when examining raw time-series data. It should be noted that many classical statistical tests depend on the assumption of normality, and when the time-series data have significant skewness and kurtosis diverges from three, the normality assumption is no longer valid. A brief review on the first four moments will be given in this subsection. 
(a) Channel 2

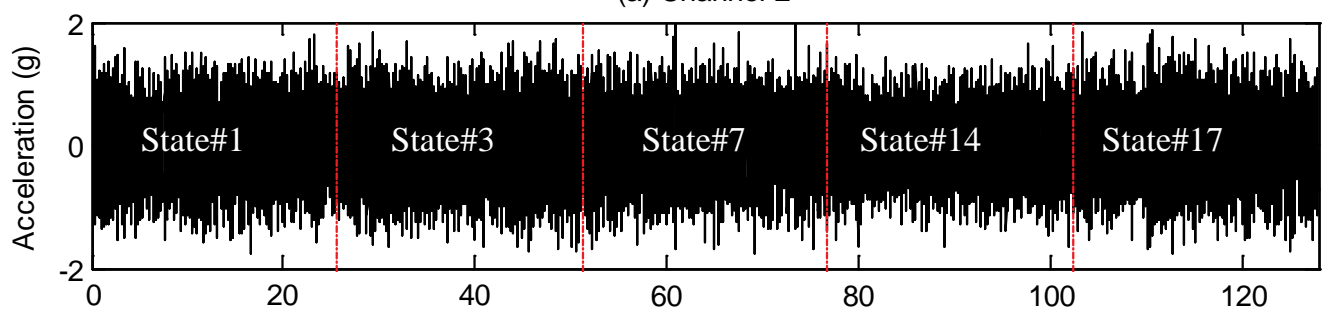

(b) Channel 3

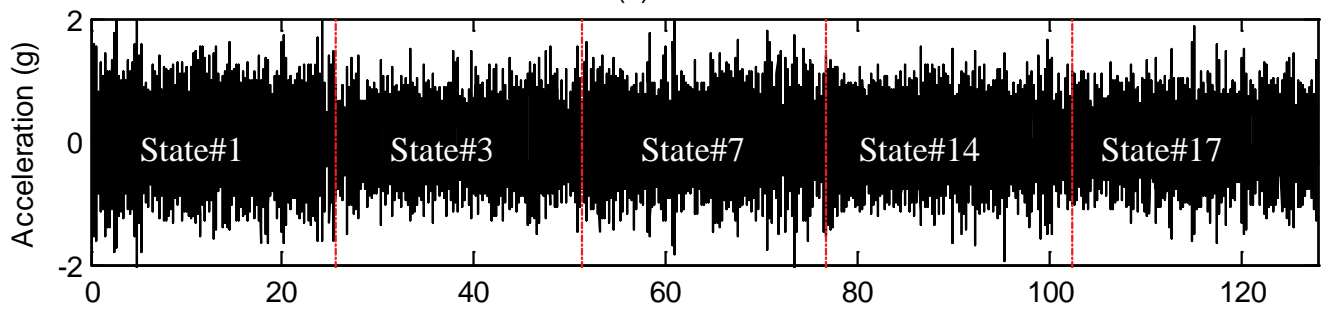

(c) Channel 4

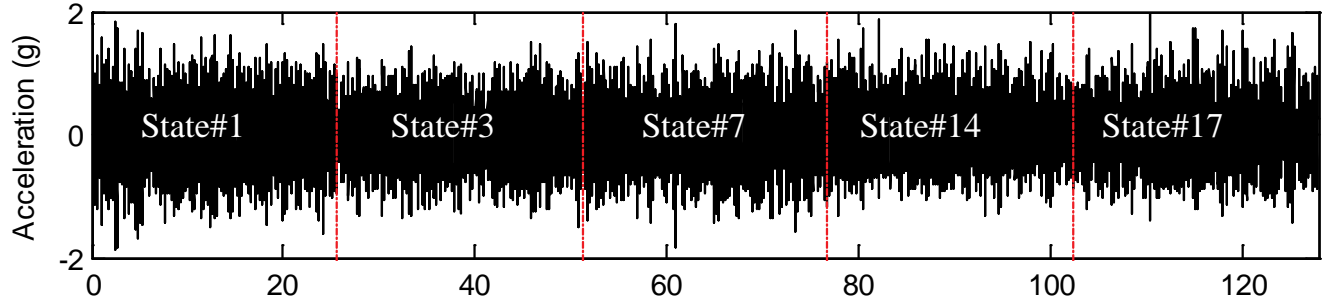

(d) Channel 5

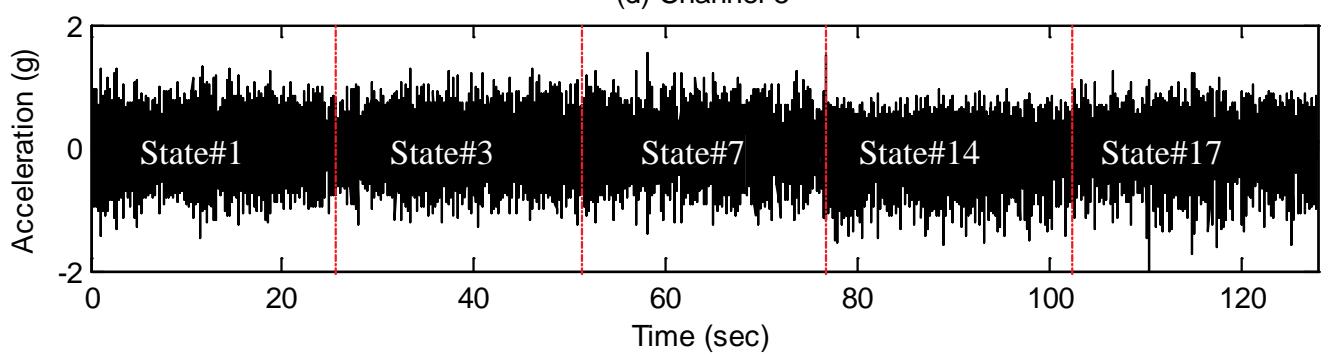

Figure 20: Acceleration-time histories of various state conditions from Channels 2 to 5.

When a random variable $X$ is measured in terms of deviations from its mean, its expectation yields moments about the mean, also referred to as central moments. The $n^{\text {th }}$ central moment of the probability distribution of a random variable $X$ is defined as

$$
\mu_{n}=E\left(X-\mu_{X}\right)^{n},
$$

where $\mu_{X}=E(X)$ is the mean of $X$. The mathematical operator $E(X)$ is the expectation of the random variable $X$, i.e., in simple terms, the expectation operator calculates the mean of a random quantity. Thus, the first central moment of a standard normal distribution is zero, because the first moment of a symmetric distribution about the mean is zero.

The variance is the second central moment. The variance of a random variable, $\sigma_{X}^{2}$, is a measure of the dispersion from the mean, and is defined as

$$
\sigma_{X}^{2}=E\left(X-\mu_{X}\right)^{2} \text {. }
$$


The square root of the variance is called the standard deviation and is denoted as $\sigma_{X}$. Although the mean describes the central tendency of the data, the standard deviation describes the spread about the mean.

The third statistical moment is a measure of the asymmetry of the PDF. The normalized third statistical moment is called the skewness, $S$, and is defined as

$$
S=\frac{E\left(X-\mu_{X}\right)^{3}}{\sigma_{X}^{3}}
$$

where a positive skewness means that the right tail is longer and that the area of the distribution is concentrated below the mean. On the other hand, a negative skewness means that the left tail is longer and that the area of the distribution is concentrated above the mean. The skewness of a standard normal distribution is zero.

The fourth statistical moment is a measure of the relative amount of data located in the tails of a probability distribution. The kurtosis, $k$, is the normalized fourth statistical moment and is defined as

$$
k=\frac{E\left(X-\mu_{X}\right)^{4}}{\sigma_{X}^{4}},
$$

where a kurtosis greater than three indicates a "peaked" distribution that has longer tails than a standard normal distribution. This means that there are more cases far from the mean. Kurtosis less than three indicates a "flat" distribution with shorter tails than a standard normal distribution. This property implies that fewer realizations of the random variable occur in the tails than would be expected in a normal distribution. The kurtosis of a standard normal distribution is three.

The $k^{\text {th }}$ central moment about the mean, $m_{k}$, for a sample $x_{1}, \ldots, x_{n}$ with $n$ observations from a random process $X$ is defined as

$$
m_{k}=\frac{1}{n} \sum_{i=1}^{n}\left(x_{i}-\bar{x}\right)^{k}, \quad k=1,2, \ldots
$$

where $\bar{x}=m_{1}$ is the sample average.

In statistics, it is common to use a standard data normalization procedure in the form of

$$
z_{j}^{n}=\frac{x_{j}^{n}-\bar{X}_{j}}{s_{j}},
$$

where $n$ are the observations, $\bar{x}_{j}$ is the mean, and $s_{j}$ is the standard deviation of each sample $j$. Thus, the normalized sample given by $z_{j}^{n}$ has a zero mean and a unit standard deviation.

Figure 21 shows the first four statistical moments of one time history from Channels 2-5 of each state condition. The mean and standard deviation of the time histories does not give any insight about the presence of nonlinearities associated with the damaged state conditions (States \#10-\#17). However, the skewness and kurtosis show some differences in the damaged states when compared to the undamaged states conditions (States \#1-\#9). 

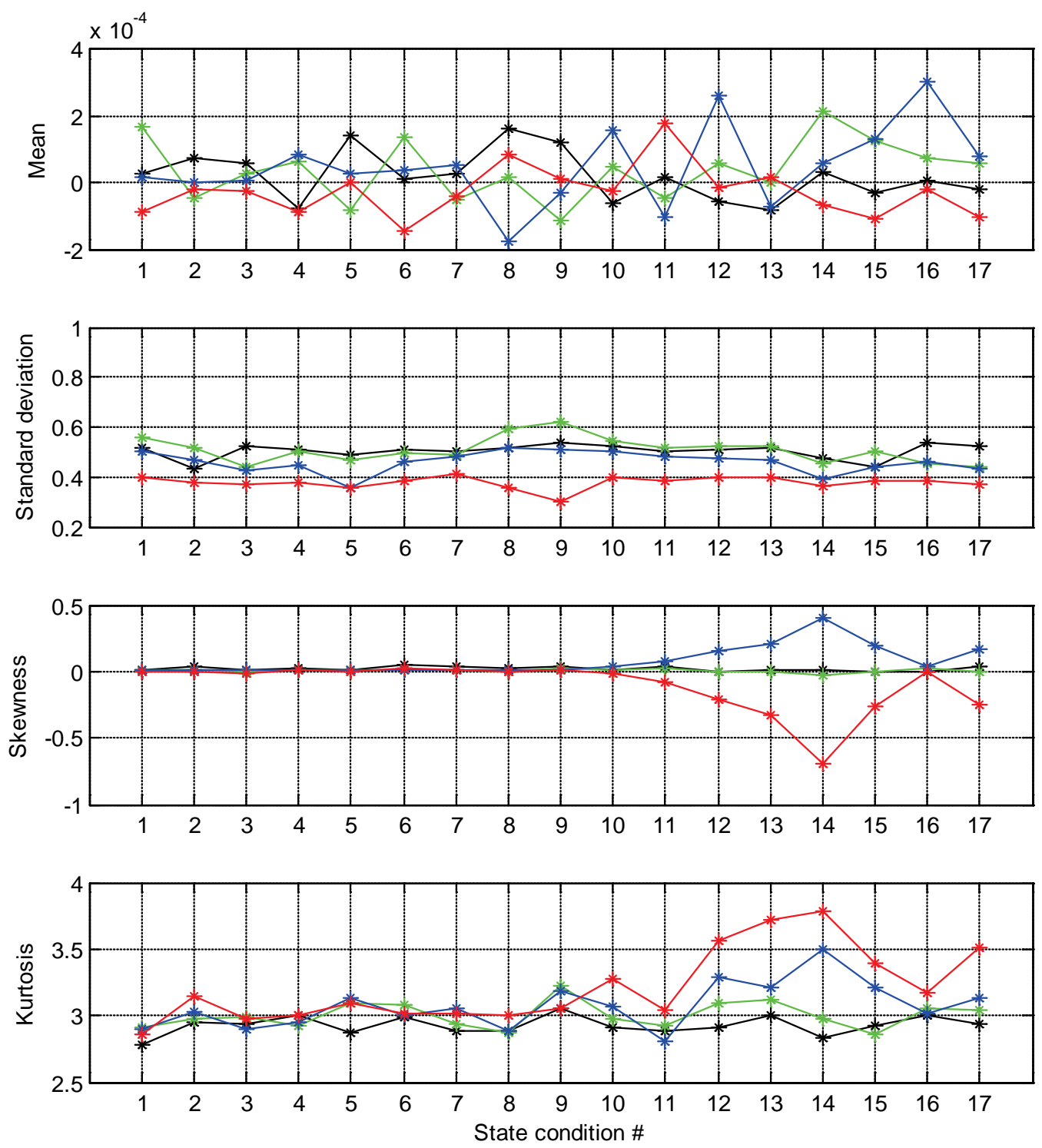

—* Channel $2 \div$ Channel $3 \multimap$ Channel $4 \div$ Channel 5

Figure 21: First four statistical moments for each state condition.

From Figure 21 it is clear that for almost all of the damaged states, the skewness diverges from zero, with the possible exception to State \#16. It is of interest to note that the skewness has an opposite sign for the sensors on either side off the impact device, implying that the response from Channel 4 has more values below the mean in its damaged condition, and the response from Channel 5 has more values above the mean. Moreover, these same damaged states in general have larger kurtosis (larger than 3.2) than the undamaged states. Note that a kurtosis larger than three means that most of the variance is caused by nonfrequent extreme deviations from the mean. However, for both skewness and kurtosis, the changes are only significant in the data from Channel 4 and 5, which are on the floors directly above and below the bumper location. This fact points out the challenge of using sensors to detect sources of damage far from the damage locations. 
In conclusion, the skewness and kurtosis can be used as features to detect damage that results in a linear system subsequently exhibiting nonlinear dynamic response and when the damage introduces an asymmetry into response data. In these cases, the sensors had to be located close to the damage.

\subsubsection{Probability Density Distribution}

Relevant statistical changes in the acceleration-time histories from Channel 5, that are the result of damage, can be observed in the estimated PDFs obtained from data corresponding to the structure in its different damaged conditions. As stated above, the measured data of the baseline condition are assumed to be normally distributed. Based on this assumption, the normal probability plots are used to graphically verify whether the measured data are from a normal distribution. The normal probability plot will be linear when the data come from a normal distribution. If the data are related to another probability distribution, they will introduce curvature in the plot.

Figure 22 shows normal probability plots of State \#1 (baseline), State \#3 (1.2 kg added mass on the first floor), State \#14 (gap of $0.05 \mathrm{~mm}$ ), and State \#17 (a gap of $0.1 \mathrm{~mm}$ and $1.2 \mathrm{~kg}$ added on the first floor). The nonlinear scale of the vertical axis corresponds to empirical probability (from 0 to 1 ) versus the accelerations data points in the horizontal axis. In a normal probability plot, all points should fall close to the dashed line if the data are normally distributed. Thus, the plots clearly show that data from the baseline condition (State \#1) and the state affected by simulated operational and environmental conditions (State \#3), but without nonlinearities, are normally distributed. Note that in this case the shaker does not have a feedback control mechanism and as such has difficulty in reproducing an accurate Gaussian input. This difficulty manifests itself in the tails of the distribution where slight deviations from the normal condition can be seen. However, the states with the nonlinearities (States \#14 and \#17) show that an assumption of normality is not justified, as shown by the significant curvature in the tails of the normal probability plot.

Another technique to verify the underlying distribution of the data is to estimate its PDF using nonparametric density estimators. In statistics, the histogram density estimator and the kernel density estimator are two techniques used to estimate an unobservable underlying PDF of a random variable. Although the former is the oldest and most widely used form of density estimation, in this study only the latter is used.

The kernel density estimator is a generalization and improvement over histograms. Given data from a sample of a population, the kernel density estimator makes it possible to draw inferences about the entire population being studied. A very brief summary of the approach will be given here for completeness. More details about this estimator can be found in the references (Silverman, 1986).

Considering a series from a random variable $X$ with $n$ observations, $x_{1}, \ldots, x_{n}$, the estimated density distribution, $\hat{f}(x)$, is given at any point $x$ by

$$
\hat{f}(x)=\frac{1}{n h} \sum_{i=1}^{n} K\left(\frac{x-x_{i}}{h}\right),
$$

where $K$ is the kernel function and $h$ is the bandwidth, also called the smoothing parameter. Even though there are many choices among kernels, in this study, $K$ is taken to be a standard Gaussian function with a zero mean and unit variance as follows: 


$$
K(x)=\frac{1}{\sqrt{2 \pi}} e^{-\frac{1}{2} x^{2}}
$$

(a) State\#1

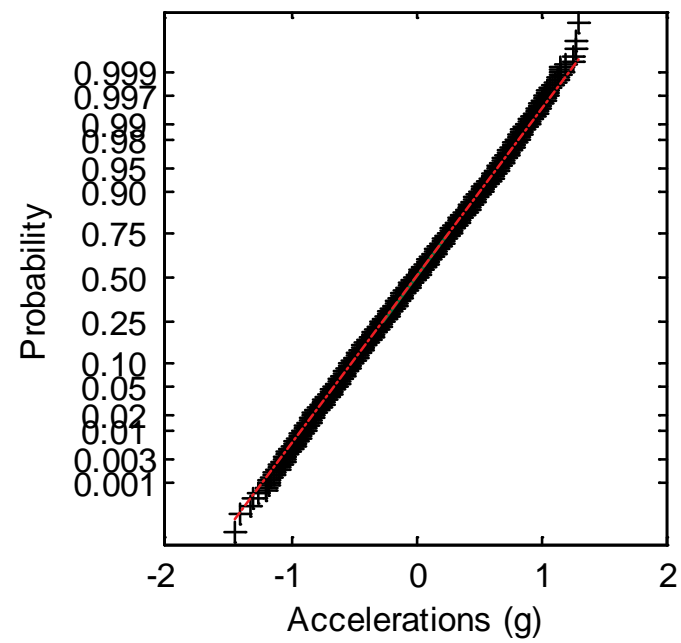

(c) State\#14

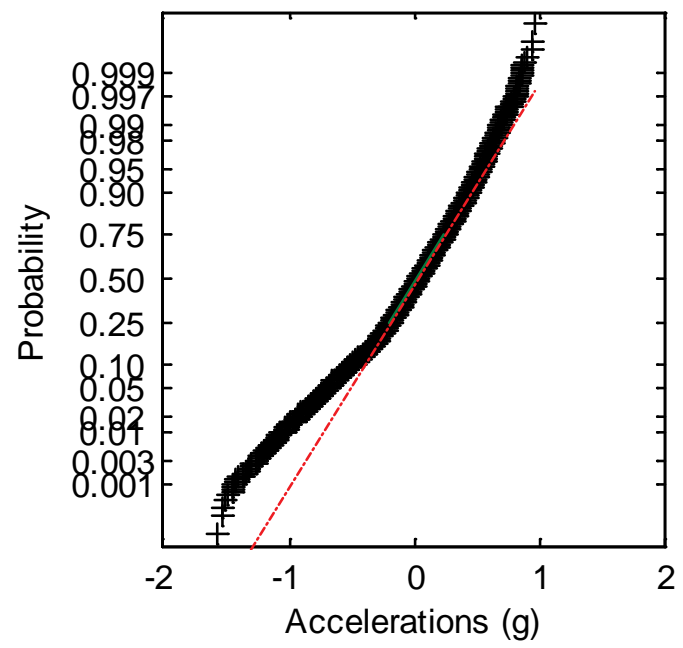

(b) State\#3

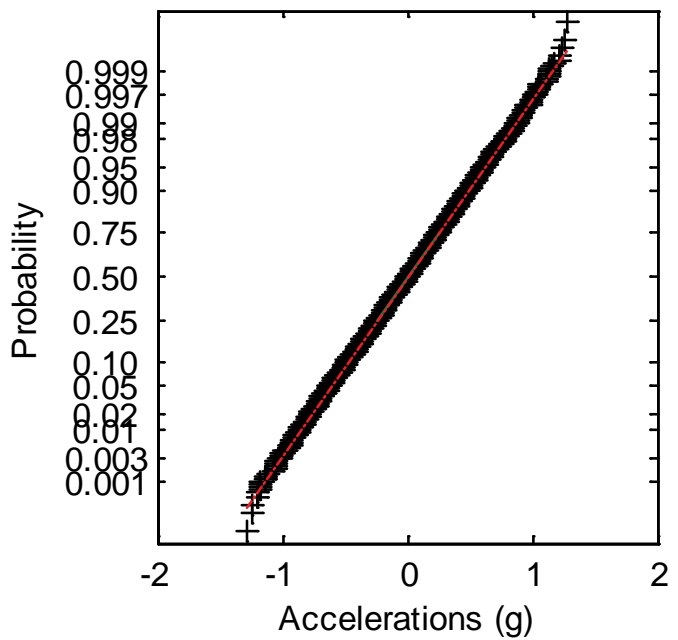

(d) State\#17

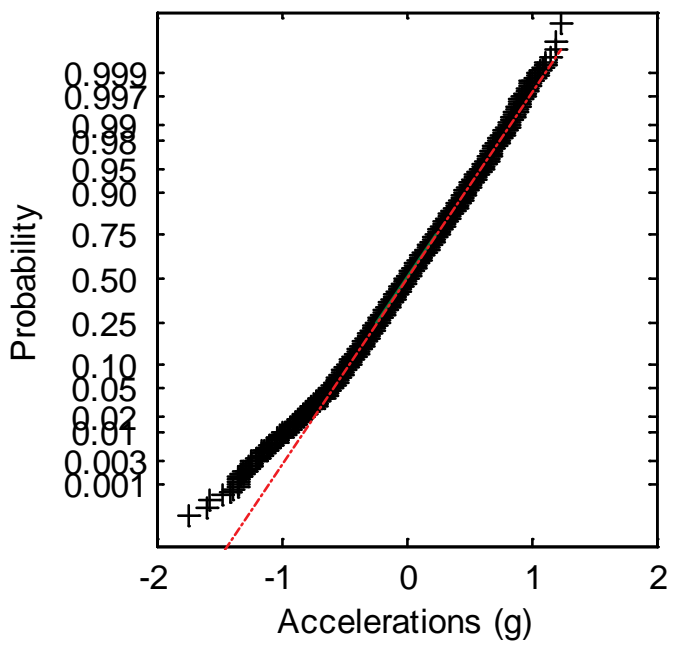

Figure 22: $\quad$ Normal probability plots of four state conditions for Channel 5.

One of the most important factors for a good kernel density estimator is the choice of the smoothing parameter $h$. The quality of the kernel estimate depends less on the shape of the kernel function than on the value of its bandwidth. A value $h$ that is too small or too large is not useful because small values of $h$ lead to spiky estimates, and the kernel density estimate is said to be undersmoothed. On the other hand, large values of $h$ lead to flat estimates and the kernel density estimate is said to be oversmoothed. In the SHM field, the definition of this parameter tends to be crucial because damage often manifests itself as small changes in the tails of the distribution. Several techniques to estimate the optimum value for $h$ can be found in the references (Browman, 1984).

Figure 23 shows the individual estimates of the PDFs for Channel 5 of States \#1, \#3, \#7, $\# 14$, and \#17 using the kernel density estimator. This estimation is based on the normal kernel 
function given by Eq. (16) and using a smoothing parameter $h$ that is a function of the number of points in each time history. The density is estimated at 100 equally spaced points that cover the range of acceleration amplitudes in each time history. Based on the results shown in Figure 23, one can conclude that there exists a difference between the PDF of damaged State \#14 and the other state conditions.

From Figure 21 and Figure 23, it can be seen that: (i) the PDFs of States \#14 and \#17 have negative skewness; (ii) State \#14 has more nonfrequent values in the tails than the other states as indicated by its large value of kurtosis; (iii) as the level of damage increases, the PDFs of the corresponding state conditions deviate from a normal distribution.

In conclusion, it follows from analysis of the PDF estimates that the baseline condition is normally distributed. However, as the level of damage increases, the PDFs deviate from a normal distribution.

\subsection{Time-Series Analysis}

Time-series analysis takes into account the fact that data points taken over time may have an internal structure, such as autocorrelation, trend, or seasonal variation (NIST/SEMATECH, 2008). This subsection gives a brief overview of some damage-sensitive features extraction techniques based on time-series analysis and applies them to the measured data, with special attention being given to AR models.

\subsubsection{Autoregressive Model}

The AR model with $p$ autoregressive parameters, $\operatorname{AR}(p)$, can be written as

$$
x_{i}=\sum_{j=1}^{p} \phi_{j} x(i-j)+e_{i}
$$

where $x_{i}$ is the measured signal at discrete time index $i$, and $e_{i}$ is an unobservable random error (or residual error) at the $i^{\text {th }}$ signal value. The unknown AR parameters, $\phi_{j}$, can be estimated by using either least squares or the Yule-Walker equations (Box and Jenkins, 1976). In this report only the former is used.

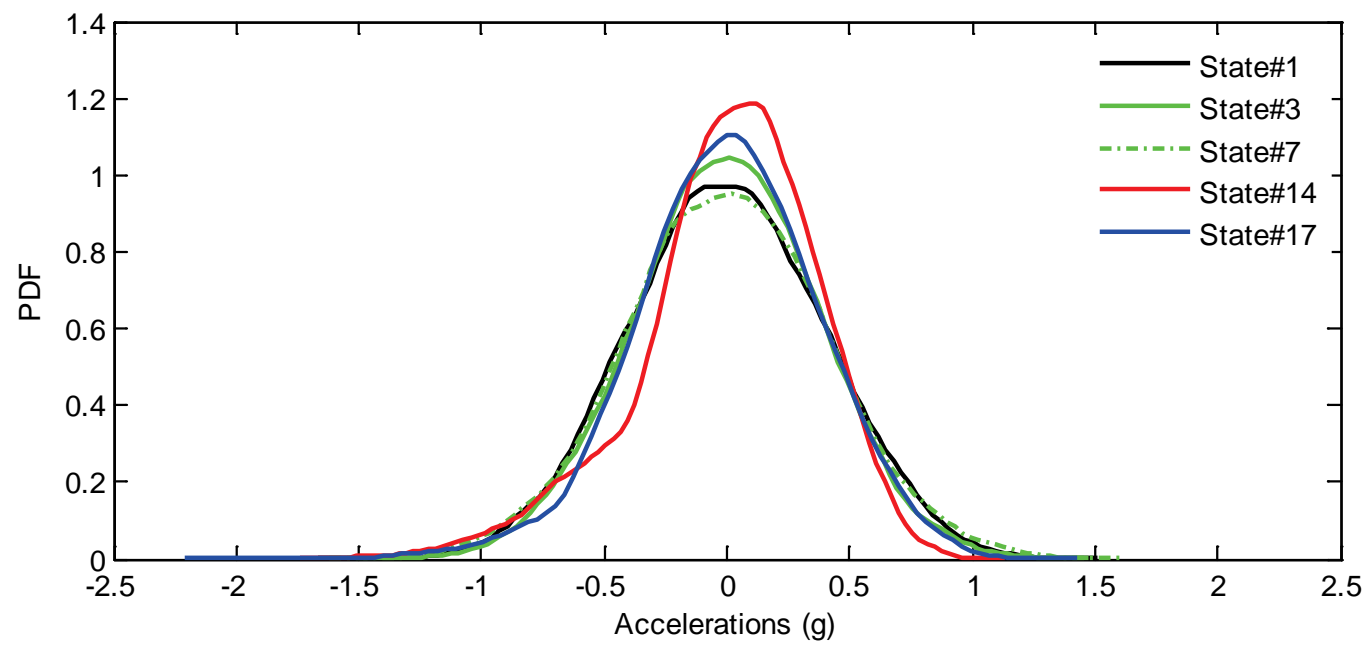

Figure 23: $\quad$ PDFs estimated by a kernel density estimator using time histories from Channel 5. 
In SHM, the AR model can be used as a damage-sensitive feature extractor based on two approaches: (1) using the AR parameters $\phi_{j}$; and (2) using the residual errors. The first approach consists of fitting an AR model to signals from undamaged and damaged structure. Then, the AR parameters $\phi_{j}$ are used as damage-sensitive features. The second approach consists of using the AR model, with parameters estimated from the baseline condition, to predict the response of data obtained from a potentially damaged structure. The residual error, which is the difference between the measured and predicted signal, is calculated at time $i$ as follows:

$$
e_{i}=x_{i}-\hat{x}_{i}
$$

where $\hat{x}_{i}$ is the predicted $i^{\text {th }}$ signal value. This approach is based on the assumption that damage will introduce either linear deviation from the baseline condition or nonlinear effects in the signal and, therefore, the linear model developed with the baseline data will no longer accurately predict the response of the damaged system. As a consequence, the residual errors associated with the damaged system will increase. Note that for a fitted AR $(p)$ model, the residual errors can only be computed for $i>p$ time points.

\subsubsection{Model Identification}

The order of the AR model is an unknown value. A high-order model may perfectly match the data, but will not generalize to other data sets. On the other hand, a low-order model will not necessarily capture the underlying physical system response. In order to find out the optimum model order, several techniques are used in this study, such as Akaike's information criterion (AIC), partial autocorrelation function (PAF), root mean squared error (RMSE) and SVD. A brief explanation of all techniques will be given for completeness. The specific goal of using several techniques is to establish upper and lower bounds for the optimum number of parameters needed to fit the data. The analysis is carried out for one acceleration-time history from Channel 5 of the baseline condition (State \#1).

\subsection{Akaike’s Information Criterion}

The AIC has been used to assess the generalization performance of linear models. In a simple way, this technique returns a value that is the sum of two terms as follows:

$$
A I C=-2 L_{m}+2 m
$$

where $L_{m}$ is the maximized log-likelihood of the residual error, and $m$ is the number of adjustable parameters in the model. It assumes a tradeoff between the fit of the model and the model's complexity. The first term is related to how well the model fits the data, i.e., if the model is too simple, the residual errors increase. On the other hand, the second term is a penalty factor related to the complexity of the model, which increases as the number of additional parameters grows (Box et al., 1994; Bishop, 1995; Everitt, 2002).

Figure 24 shows the AIC values calculated using Eq. (19) as a function of the order of AR models. The optimum AR order is associated with the minimal ACI value, i.e., smaller AIC values suggest an optimal tradeoff between minimizing the residual error while maintaining a model that will generalize to predict other data sets well. For the data from the baseline condition, the AIC values converge around a $25^{\text {th }}$ order model, giving an indication that an AR(25) model is a good start. 


\subsection{Partial Autocorrelation Function}

The coefficients from a PAF were estimated by fitting AR models of successively increasing order $p$ to the measured data and then plotting the last estimated coefficient, $\phi_{p p}$, as a function of the model order. Thus, the AR model of Eq. (17) can be rewritten as follows:

$$
X_{i}=\sum_{j=1}^{p} \phi_{p j} x(i-j)+e_{i}
$$

For an AR model of a noise-free order $p$ process, the PAF, $\phi_{k k}$, will be nonzero for $k \leq p$ and zero for $k>p$. For real-world structures with noise in the measurements, the partial autocorrelation coefficients of an actual $\operatorname{AR}(p)$ will not be zero after lags greater than $p$. Thus, it is necessary to define upper and lower bounds for which $\operatorname{AR}(p)$ will be considered zero if the coefficients are within those limits (Box and Jenkins, 1976). The idea is to look for the points of the PAF that are essentially zero. An approach based on the standard deviation error, $\sigma_{\phi}$, of the PAF is defined as follows:

$$
\sigma_{\phi}\left[\phi_{k k}\right]=\frac{1}{\sqrt{n}} \quad \mathrm{k} \geq \mathrm{p}+1,
$$

where the estimated partial autocorrelation coefficients of order $p+1$ and higher are approximately independently distributed, and $n$ is the number of observations used in fitting the AR model. Placing a confidence interval for statistical significance is helpful for this purpose. For example, assuming an approximate 95\% confidence interval for the partial autocorrelation coefficients, the limits are placed at $\pm 2 \sigma_{\phi}$.

Figure 25 shows the first 60 estimated partial autocorrelation coefficients for one time history from Channel 5 associated with State \#1 obtained by directly fitting AR models of increasing order. This PAF shows statistical significance for lags from 1 to about 25 (note that lag 0 is always 1 ). Theoretically, the next lags should be between the limits of the confidence interval set up by the dashed horizontal lines. In spite of some coefficients falling outside the limits, the PAF indicates that an AR(25) model should be appropriate.

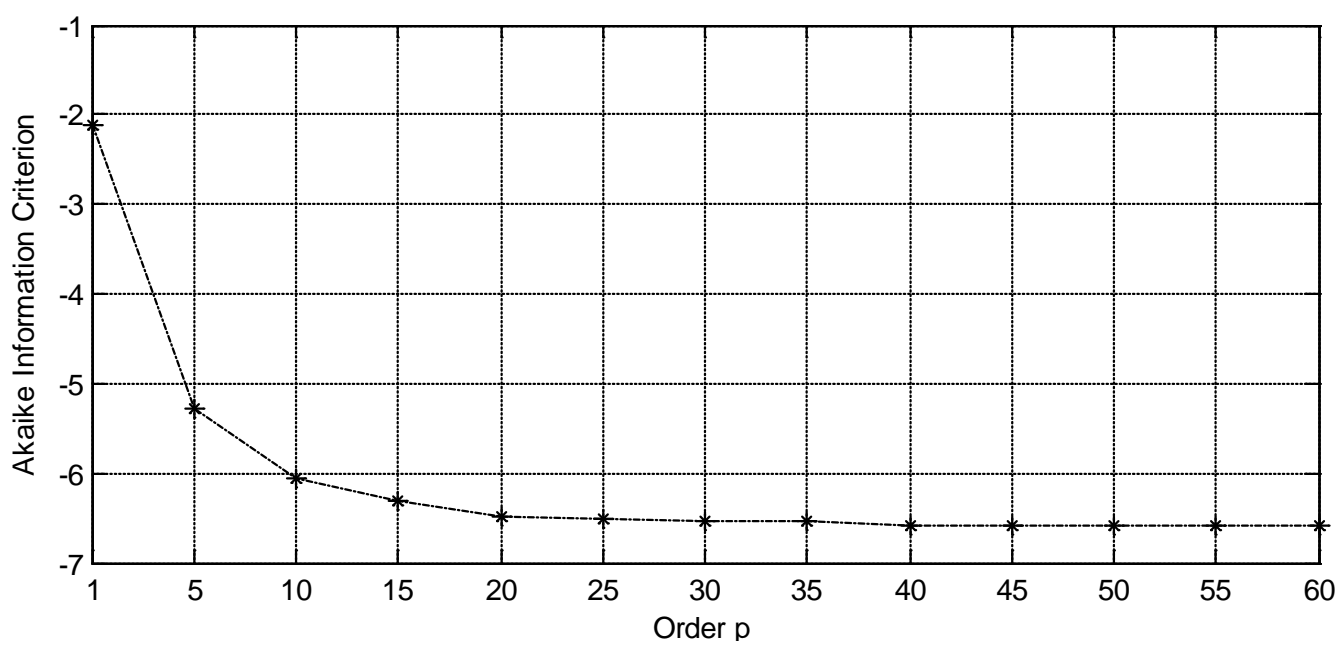

Figure 24: AIC values of AR models of increasing order (Channel 5 of State \#1). 


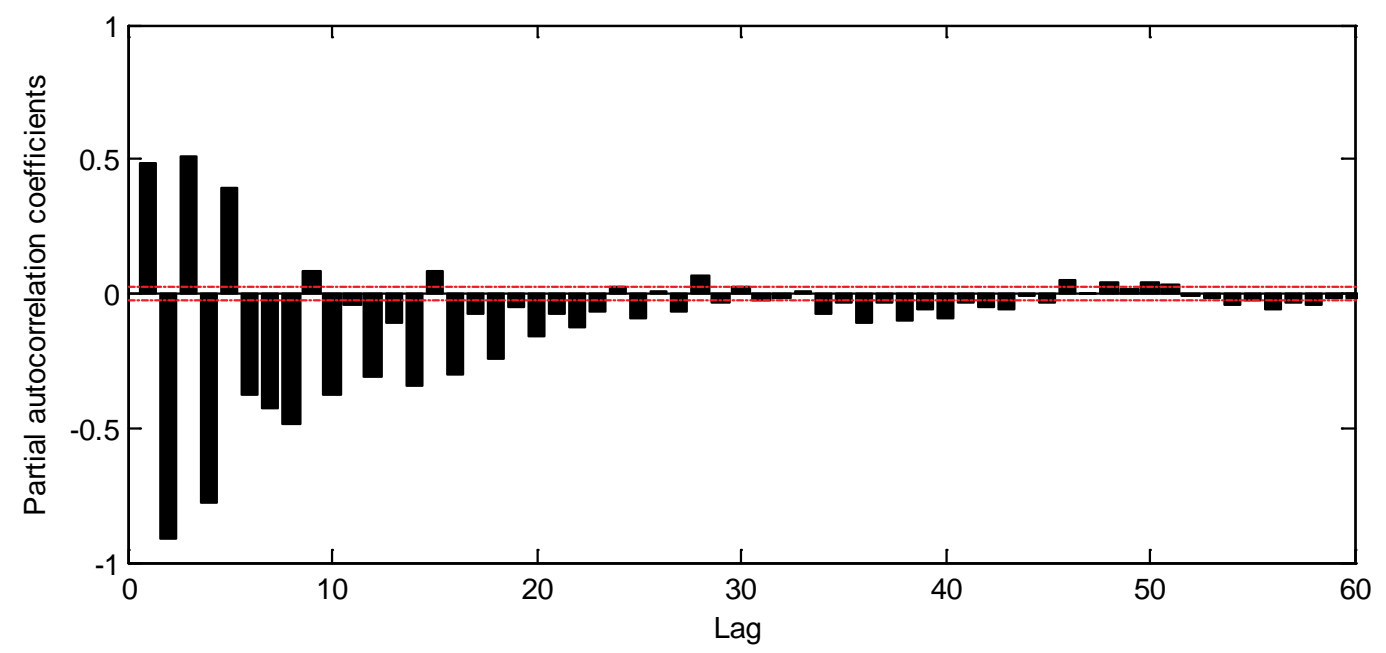

Figure 25: Estimated PAF of AR models of increasing order (Channel 5 of State \#1).

\subsection{Root Mean Squared Error}

In statistics, the RMSE of an estimator $\hat{\theta}$, with respect to the estimated parameter $\theta$, is a real value of how much the estimator differs from the estimated parameter, as follows:

$$
\operatorname{RMSE}(\hat{\theta})=\sqrt{E\left((\hat{\theta}-\theta)^{2}\right)} .
$$

In the AR model, the RMSE is a measure of the differences between values estimated by model and the values actually measured. For a time series from a random variable $X$ with $n$ observations in the form of $x_{1}, \ldots, x_{n}$, Eq. (22) becomes

$$
\operatorname{RMSE}(\hat{X})=\sqrt{\frac{1}{n} \sum_{i=1}^{n}\left(X_{i}-\hat{X}_{i}\right)^{2}}
$$

For the purpose of finding the optimum AR model, the RMSE is plotted as a function of the model order. The estimation of the AR order can be achieved by minimizing the RMSE value. Figure 26 shows the RMSE of AR models of increasing order (from 1 to 60) for one time history from Channel 5 of State \#1. The results suggest that an AR model of order $p=10$ would fit the time history well. 


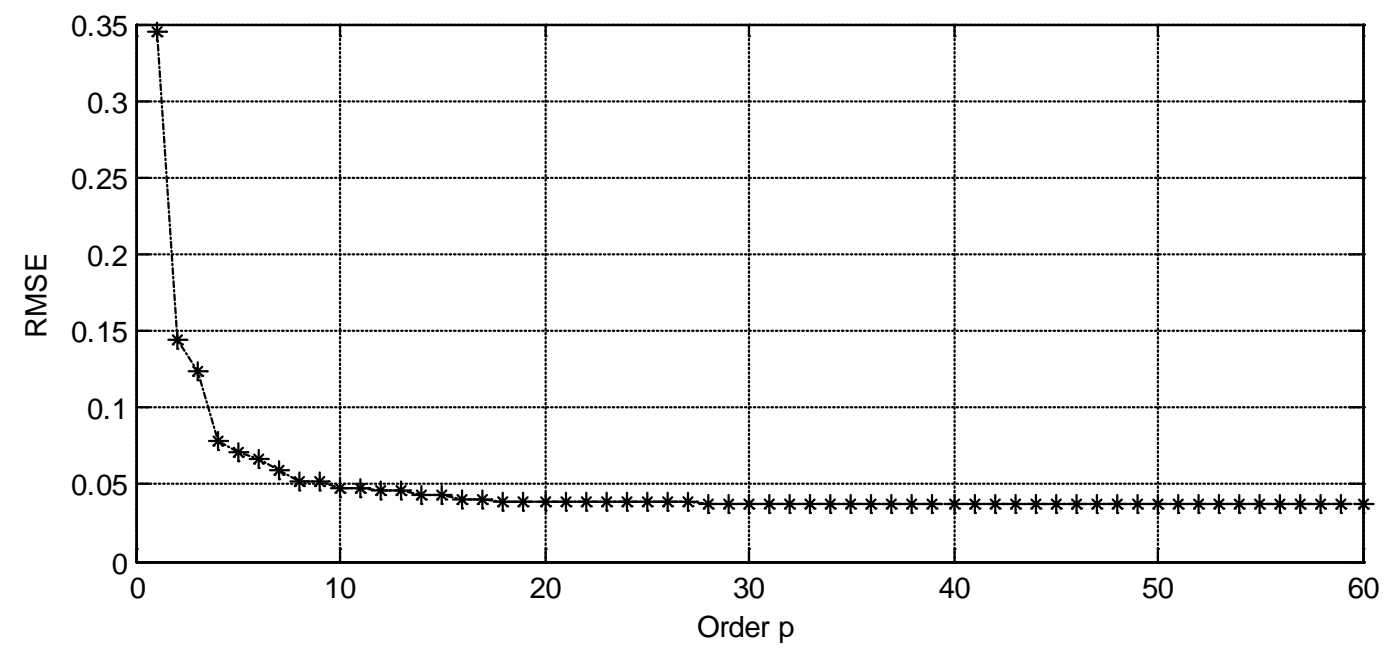

Figure 26: RMSE of AR models of increasing order (Channel 5 of State \#1).

\subsection{Singular Value Decomposition}

In linear algebra, the SVD is a factorization of a rectangular matrix, $M$, of dimension $(m \times n)$, defined as follows

$$
M=U \Lambda V^{T},
$$

where the matrix $\Lambda_{(m \times n)}$ contains the singular values on the diagonal and zeros on the off diagonal terms. The matrices $U_{(m \times m)}$ and $V_{(n \times m)}$ are square. SVD is used to determine the effective rank of a matrix $M$. In order to conduct the analysis, Eq. (17) might be written as

$$
\left\{\begin{array}{c}
x_{p+1} \\
x_{p+2} \\
\vdots \\
x_{n}
\end{array}\right\}=\left[\begin{array}{cccc}
x_{1} & x_{2} & \cdots & x_{p} \\
x_{2} & x_{3} & \cdots & x_{p+1} \\
\vdots & \vdots & \cdots & \vdots \\
x_{n-p} & x_{n-p+1} & \cdots & x_{n-1}
\end{array}\right]\left\{\begin{array}{c}
\phi_{p} \\
p_{p-1} \\
\vdots \\
\phi_{1}
\end{array}\right\} \text {, }
$$

or

$$
X=M \phi,
$$

where $X$ is the measured signal and $\{\phi\}$ contains the AR parameters. Assuming that $p=60$, the matrix $M$ has a dimension of $8,132 \times 60$. Figure 27 shows the SVD for $M$ composed by one time history from Channel 5 of State \#1. The results plotted in the figure suggest that an AR(30) model order may work well.

\subsection{Conclusions}

The four techniques used did not give consistent results for the optimum order of the AR model. The AIC and PAF suggest that an AR model of the $25^{\text {th }}$ order should be appropriate. Using the SVD technique through the effective rank of a matrix, the singular values' respective amplitude suggests that a $30^{\text {th }}$ order model should work. However, by tracking the RMSE of the 
residual errors for models of increasing order, this technique gives an indication that a $10^{\text {th }}$ order model should be appropriate. Based on this analysis, throughout this report both the AR(5) and AR(30) models will be used in order to point out the influence of the AR model order on the damage-detection process.

\subsubsection{Model Estimation}

Figure 28 and Figure 29 plot the AR(5) and AR(30) model parameters, respectively, for all the structural state conditions. The AR parameters were estimated by fitting the AR model to a time history from Channel 5 of each state condition using the least-squares technique. The parameters are assembled into groups for discrimination purposes. In the case of the AR(5) model, the damaged states are split into two groups in order to highlight the influence on the AR parameters of the operational and environmental effects (damagedOEE). The figures suggest that increasing the level of nonlinearities in the damaged states tends to decrease the amplitude of the parameters. To better clarify those changes, Figure 30 shows the magnitude of the third AR(5) model parameter for all state conditions at Channel 5. One can generally see a correlation between the amplitude and the level of nonlinearity for the damaged state conditions without simulated operational and environmental variations (States \#10-\#14), However, the amplitude of the damaged states with those variations (States \#15-\#17) have no correlation with the level of damage. These results suggest that the operational and environmental variations can introduce changes in the structural response and mask the responses related to damage. This fact makes the discrimination of the damaged states challenging with operational and environmental variations from all the undamaged states.

In conclusion, AR parameters appear to be potential damage-sensitive features. The source of damage seems to induce changes in those parameters related to the level of damage and, therefore, this feature can also be used to evaluate the severity of damage. However, the correlation with the level of damage seems to disappear when the damage is present with simulated operational and environmental variations.

After the parameters have been estimated for both AR models, these models will be analyzed to test how well the models fit the data. Figure 31 (a) and Figure 32 (a) show an overlap of the measured and estimated acceleration-time histories at Channel 5 for the baseline condition (State \#1) using the AR(5) and the AR(30) models, respectively. From a qualitative point of view windowed time histories with 50 points, shown in Figure 31 (b) and Figure 32 (b), illustrate that both the AR(5) and the AR(30) models developed from the baseline condition appear to predict the data well. 


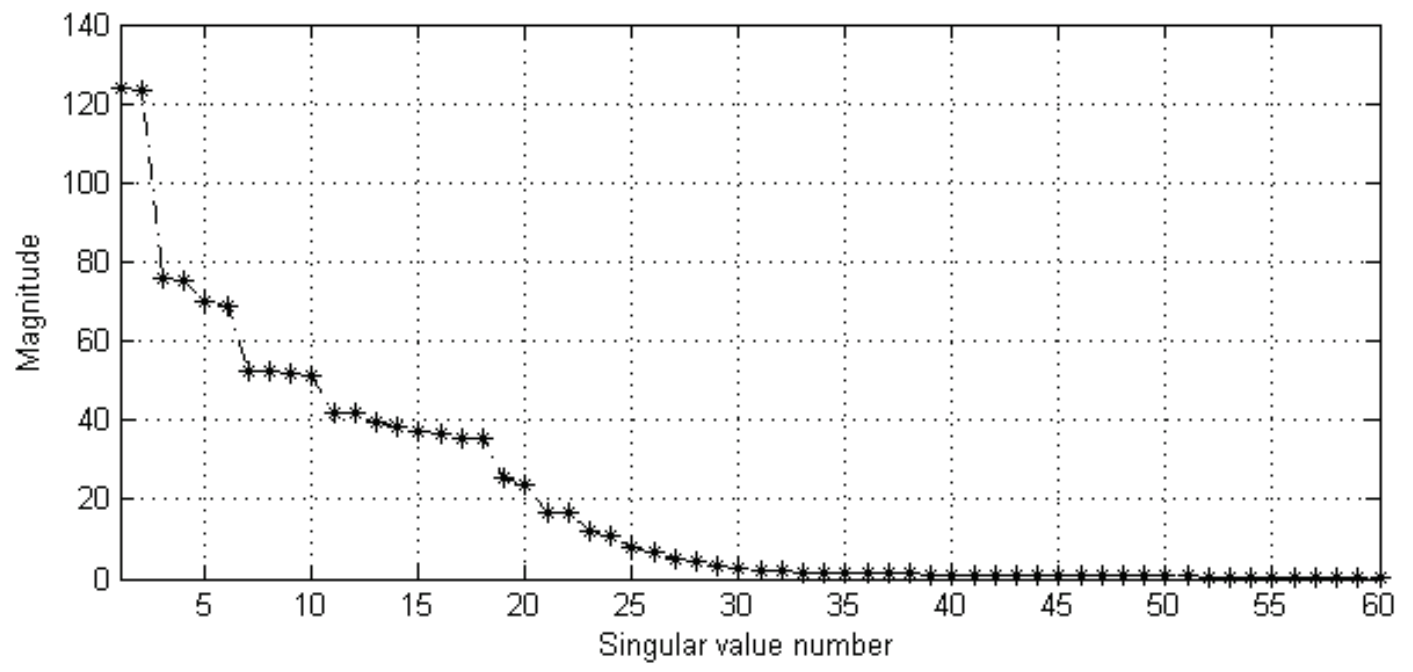

Figure 27: SVD of the AR(60) model using a time history from Channel 5 of State \#1.

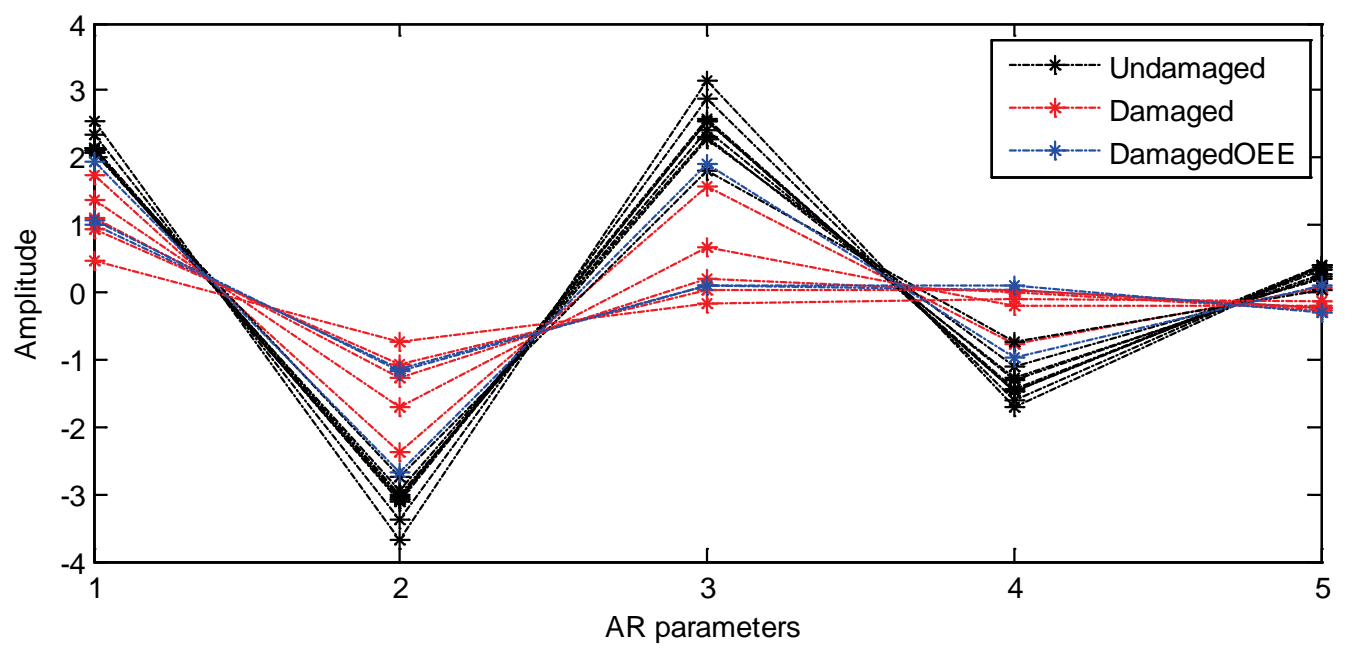

Figure 28: AR(5) model parameters for all structural state conditions (Channel 5).

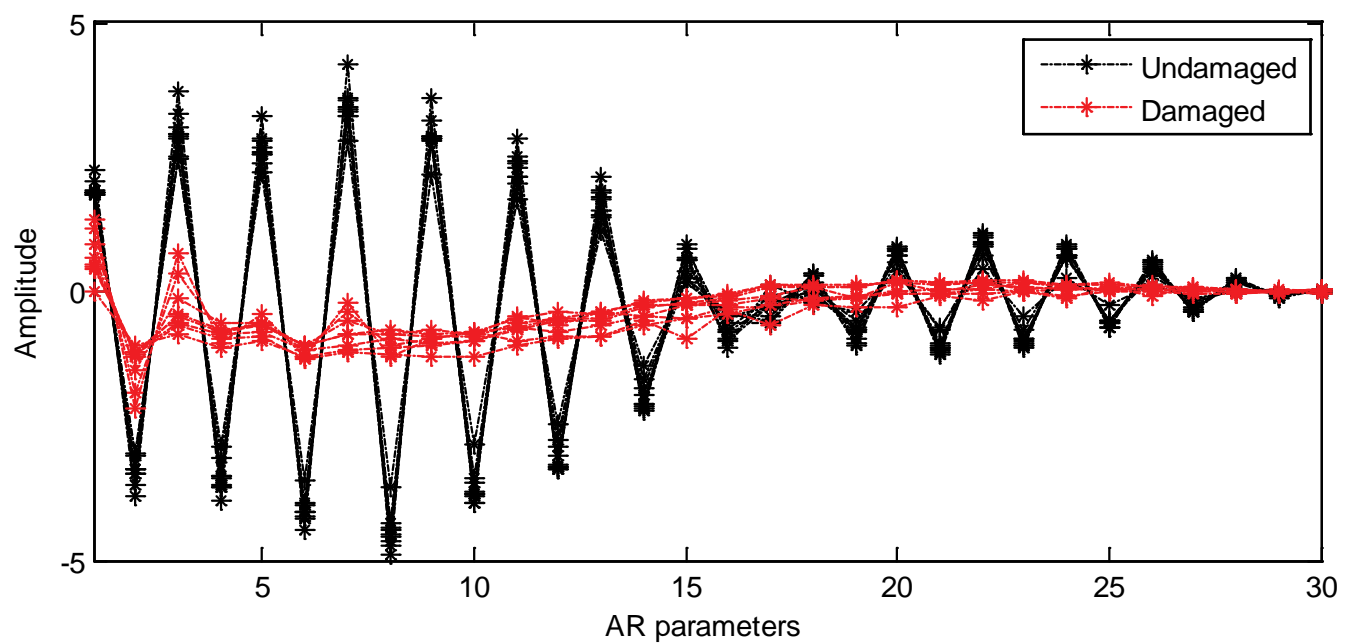

Figure 29: $A R(30)$ model parameters for all structural state conditions (Channel 5). 


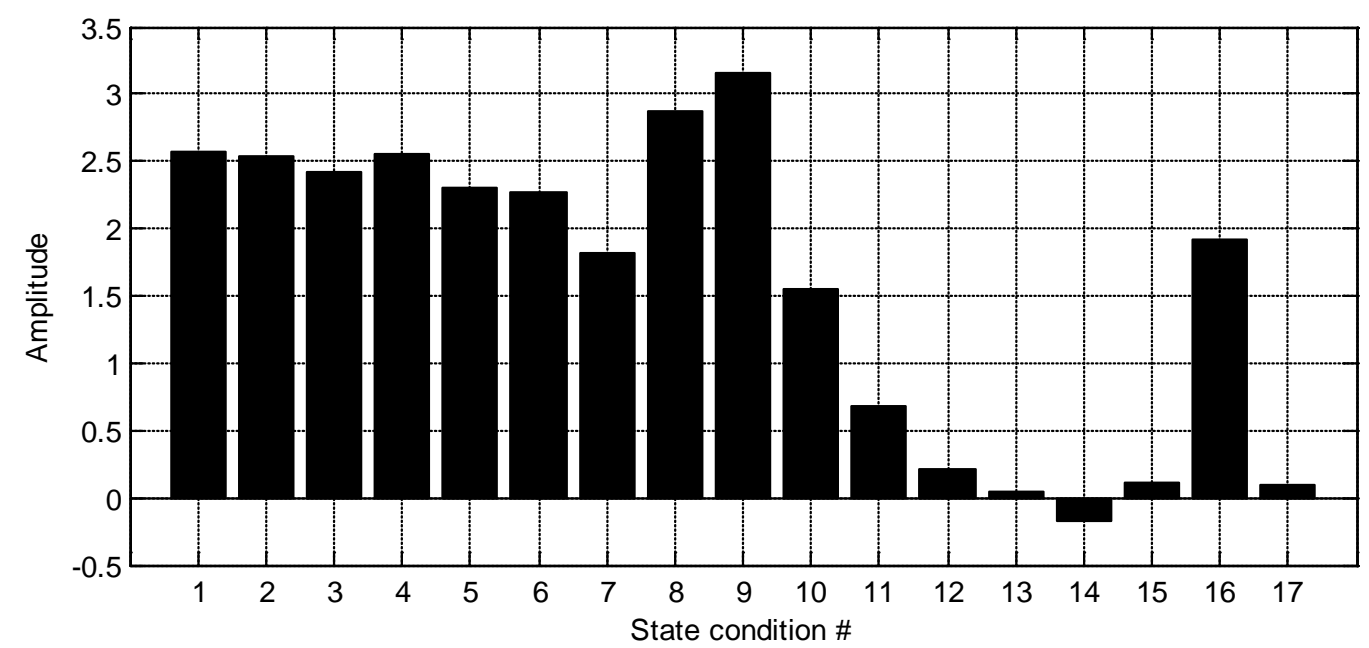

Figure 30: Amplitude of the $3^{\text {rd }}$ parameter from the AR(5) model at Channel 5 for all state conditions.

(a) Full time histories

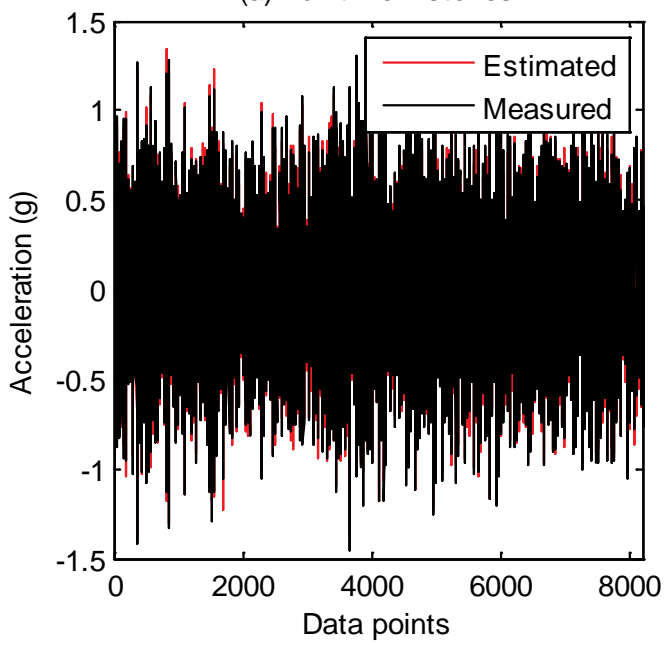

(b) Windowed time histories

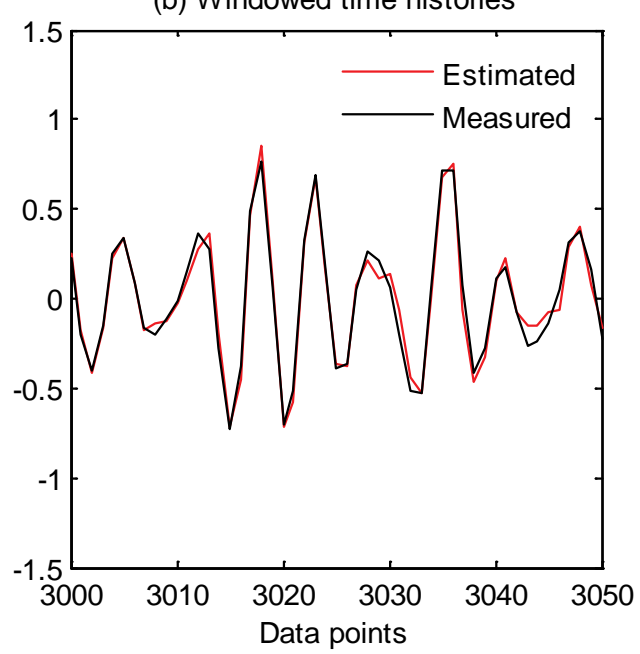

Figure 31: Comparison of the measured and estimated time histories using the AR(5) model fit to State \#1 data from Channel 5. 
(a) Full time histories

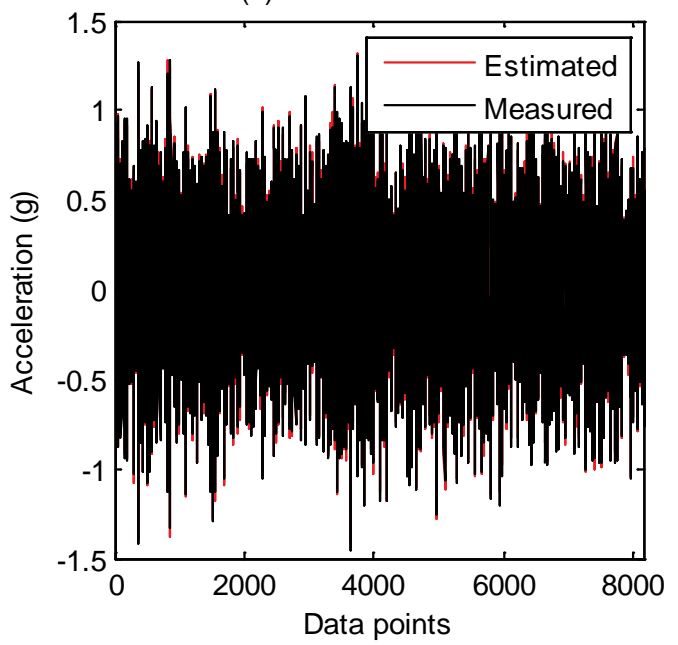

(b) Windowed time histories

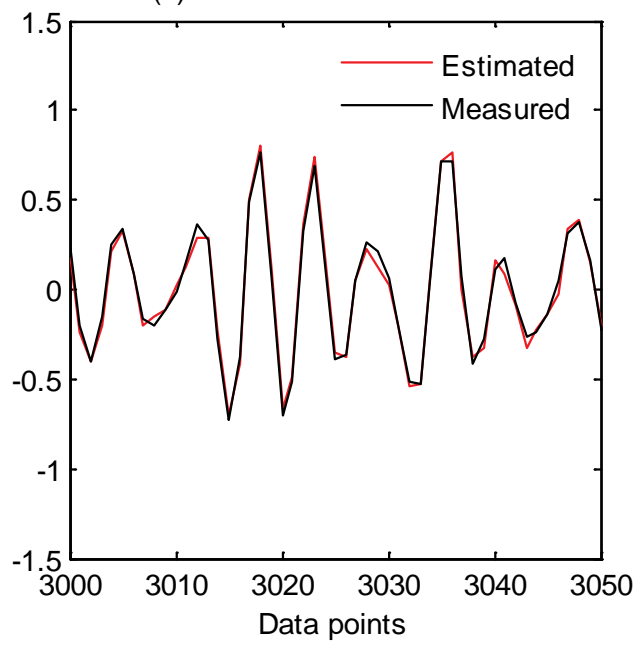

Figure 32: Comparison of the measured and estimated time histories using the AR(30) model fit to State \#1 data from Channel 5.

One indication that the AR model is fitting well the data is indicated by a Gaussiandistributed residual error. Figure 33 (a) and Figure 34 (a) show a histogram of the residual errors using 20 bins and an imposed Gaussian distribution that is based on the mean and standard deviation from these data. As it was expected, the histograms show that the higher the order of the AR model, the lower the magnitude of the residual errors. Notice that depending on the level of nonlinearity introduced by the bumper, the distribution of the residual errors may not be Gaussian when the baseline-based AR model is used to predict the response based on data from the damaged states.

The central limit theorem states that the distribution of a sum of random variables tends to be normal, even when the distributions of the individual random variables forming the sum are decidedly not normal. Therefore, one can normalize the data by forming sums of the residual errors and examining the statistical properties of these sums. This process also reduces the number of residual errors per time history that need to be stored. The process begins by first performing a standard data normalization. For each residual error time history given by Eq. (18), the residual errors mean, $\mu_{e}$, is subtracted from each value $i$, and the result is divided by the residual errors standard deviation, $S_{e}$, resulting in a standard normalization procedure (recall Eq. (14)) defined as

$$
Z_{i}=\frac{e_{i}-\mu_{e}}{S_{e}}
$$

where $z$ is the normalized residual errors vector. Next, the normalized residual errors are grouped into 4-sample data blocks, and the mean of each data block is calculated. The mean values are the new damage-sensitive features. For the AR(5) model, this process reduces the residual error features from 8187 individual values to 2046 subgroups of residuals. Note that the residual errors are computed from accelerations measurements in the range of 6 to 8192, and for convenience the last three residuals are discarded. For both models, the transformed residual errors are shown in Figure 33 (b) and Figure 34 (b), along with the normal distribution based on the mean and standard deviations of these mean values.

Even though the residual errors of both models, apparently, have an underlying normal distribution, other techniques might be used to verify the data independency. In this study, the 
PSD function of the residual errors is used to find the correlation or the presence of periodic signals buried under noise. Figure 35 and Figure 36 plot the PSD of the residual errors from both $\mathrm{AR}(5)$ and $\mathrm{AR}(30)$ models. Notice that the residual errors are estimated from one time history from Channel 5 corresponding to State \#1. The plots show that the resulting residuals from the AR(5) model are correlated, because it is still possible to identify the three natural frequencies estimated, in Section 3, at 30.7, 54.2, and 70.7 Hz.

(a) Histogram of residual errors

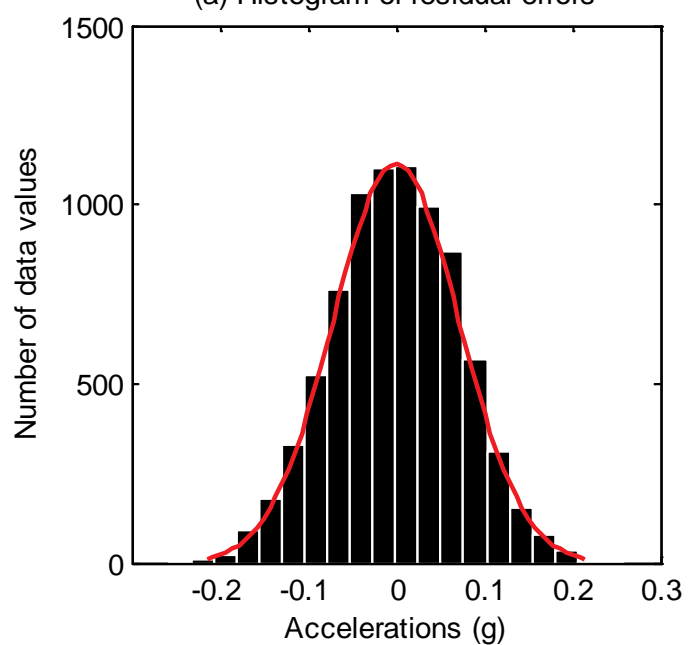

(b) Histogram of data block means

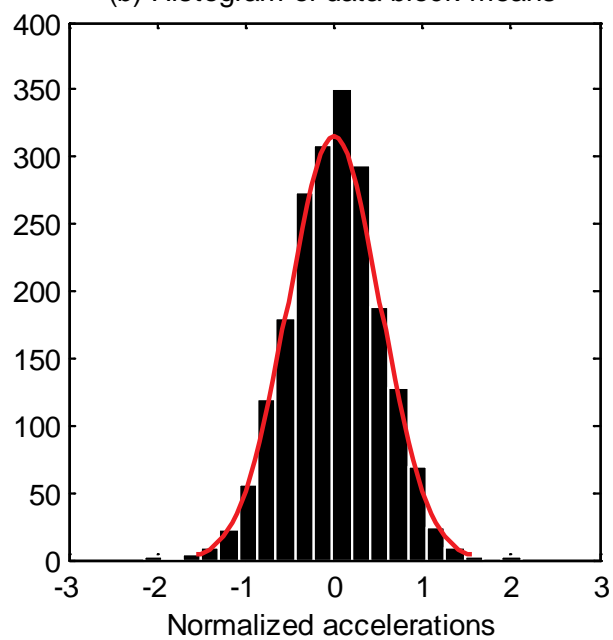

Figure 33: $A R(5)$ residual errors histograms with the corresponding Gaussian distribution (Channel 5, State \#1).

(a) Histogram of residual errors

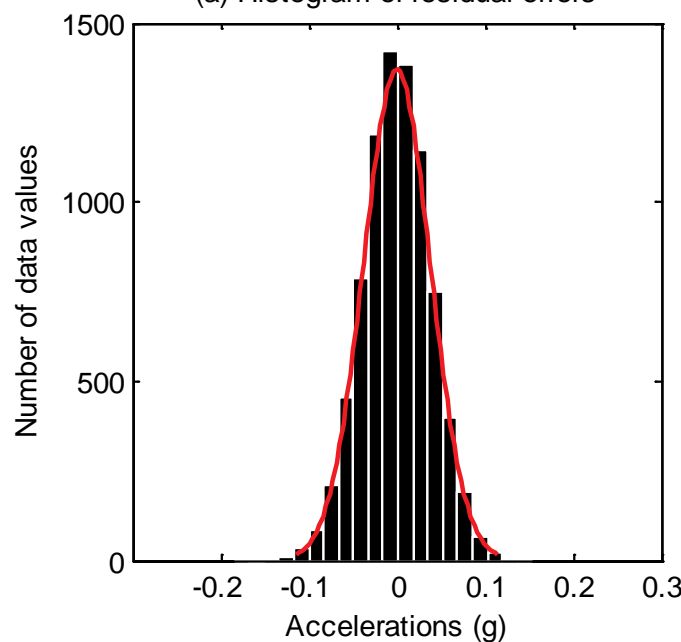

(b) Histogram of data block means

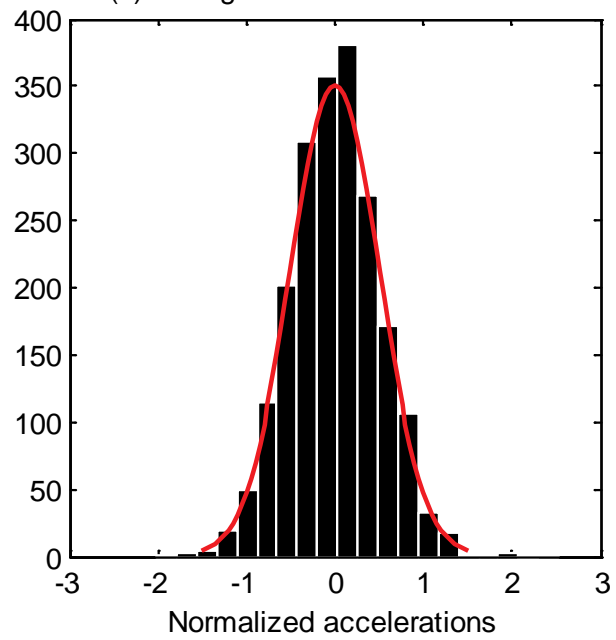

Figure 34: AR(30) residual errors histograms with the corresponding Gaussian distribution (Channel 5, State \#1). 


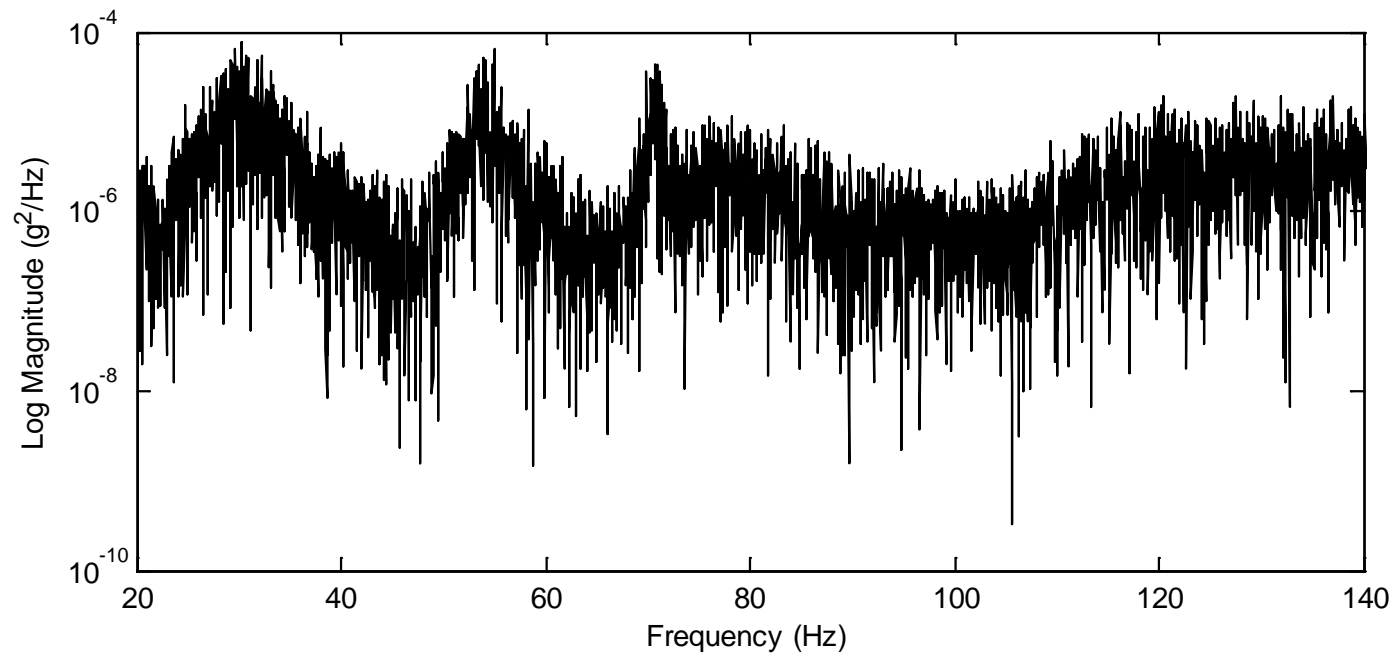

Figure 35: Log PSD of the AR(5) model residual errors (Channel 5, State \#1).

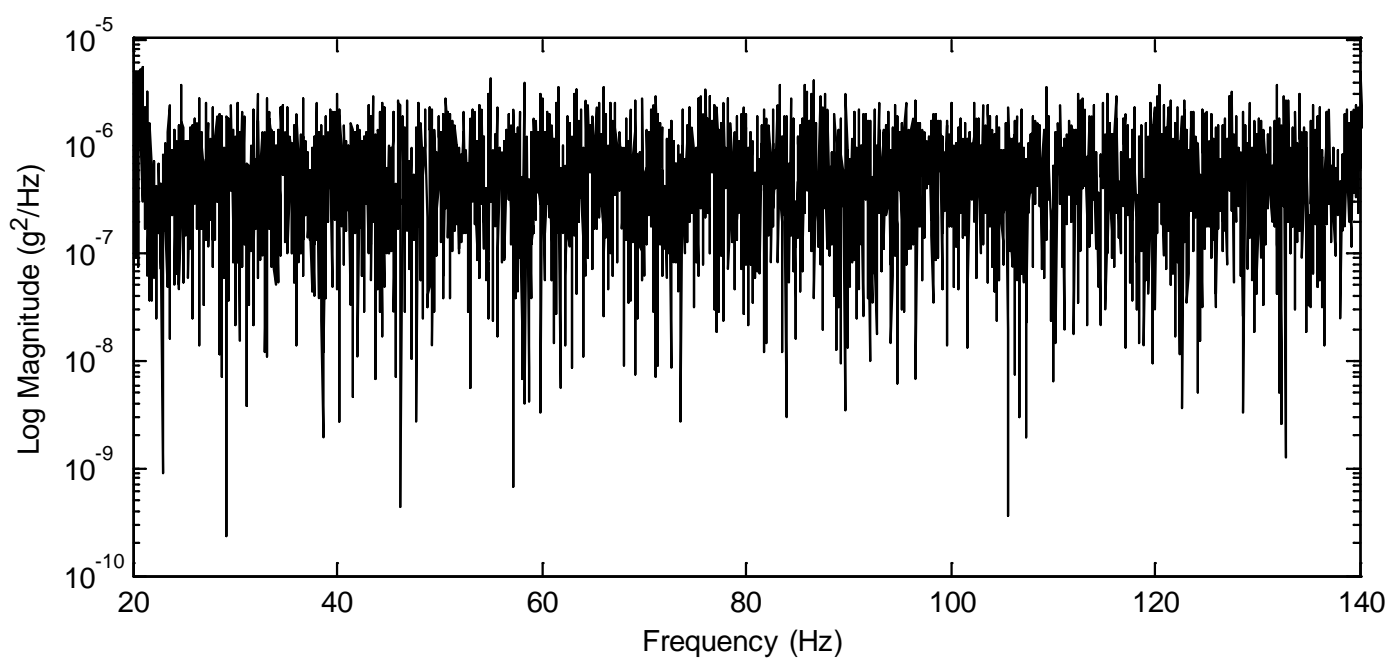

Figure 36: $\quad$ Log PSD of the AR(30) model residual errors (Channel 5, State \#1).

The previous analysis suggests that the AR(30) model indicated by the SVD technique is appropriate, because the residual errors are independent and normally distributed when the baseline response is calculated with parameters estimated from the same condition. The next step is to use the baseline-based AR model to predict data from potential damaged states. If some anomalies are present in the system, the residual errors will increase as discussed in Section 4.2.1. Note that this approach is based on the assumption that anomalies will introduce either linear deviation from the baseline condition or nonlinear effects in the signal and, therefore, the linear model developed with the baseline data will no longer accurately predict the response of the damaged system. As a consequence, with such indications, a next action might be to launch a full investigation to identity the cause of such anomalies. Note that those anomalies can result from either operational and environmental effects or damage. In order to discriminate those anomalies caused by damage from those anomalies caused by operational and 
environmental variability, it is postulated that the damage can introduce underlying nonrandom patterns in the measured data.

In order to find repeating patterns within the time histories, such as the presence of periodic signals buried under noise, correlation analysis is carried out using Eq. (8). Figure 37 shows the autocorrelation functions of the original acceleration-time histories from Channel 5 of State \#1 (baseline), State \#7 (under environmental variations), State \#14 (highest level of damage), and State \#17 (under operational variations and damage) using the first 200 coefficients. The strong correlation suggested by the autocorrelation functions is an indication of repeating patterns within time histories. Moreover, from these figures, it is difficult to make any inferences about the structural condition, such as the presence of periodic signals buried in the noise that is introduced by the nonlinearites.

Figure 38 shows the autocorrelation functions of the residual errors from the AR(5) model of the four states mentioned above. Theoretically, if the AR model accurately represents the original time histories, the residual errors should be nearly uncorrelated. Looking at the plots, one can conclude that the residual errors from the AR(5) model are still correlated. However, Figure 39 indicates that increasing the AR order reduces the correlation among the residual errors for the undamaged state conditions and, at the same time, it points out patterns in the residual errors when damage is introduced by the bumper. These patterns are not present in the undamaged time histories. This fact points out the need to choose an appropriate AR model order that captures the damage-related information contained in the signal.
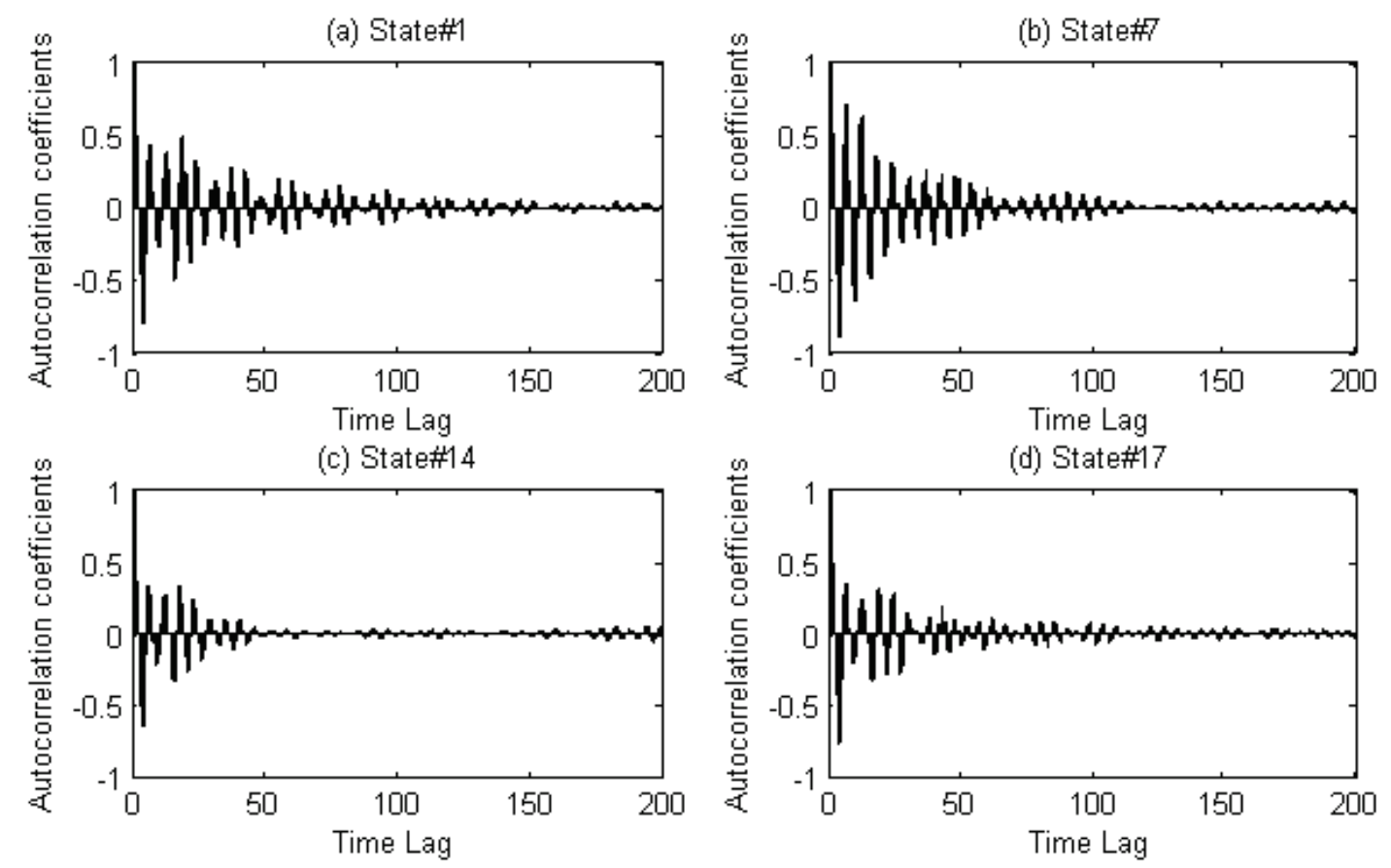

Figure 37: Autocorrelation functions of the original time histories from Channel 5. 

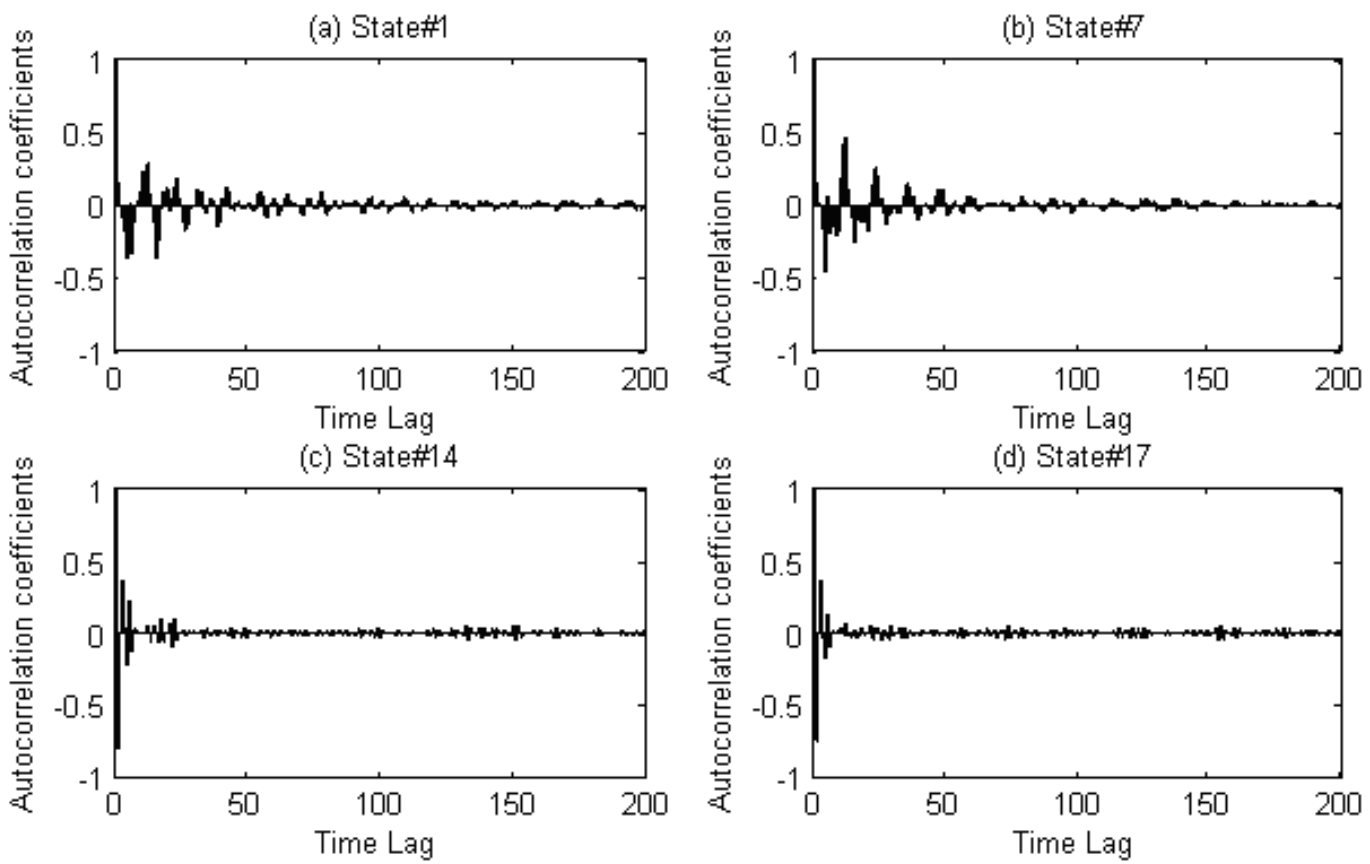

Figure 38: Autocorrelation functions of the AR(5) residual errors.
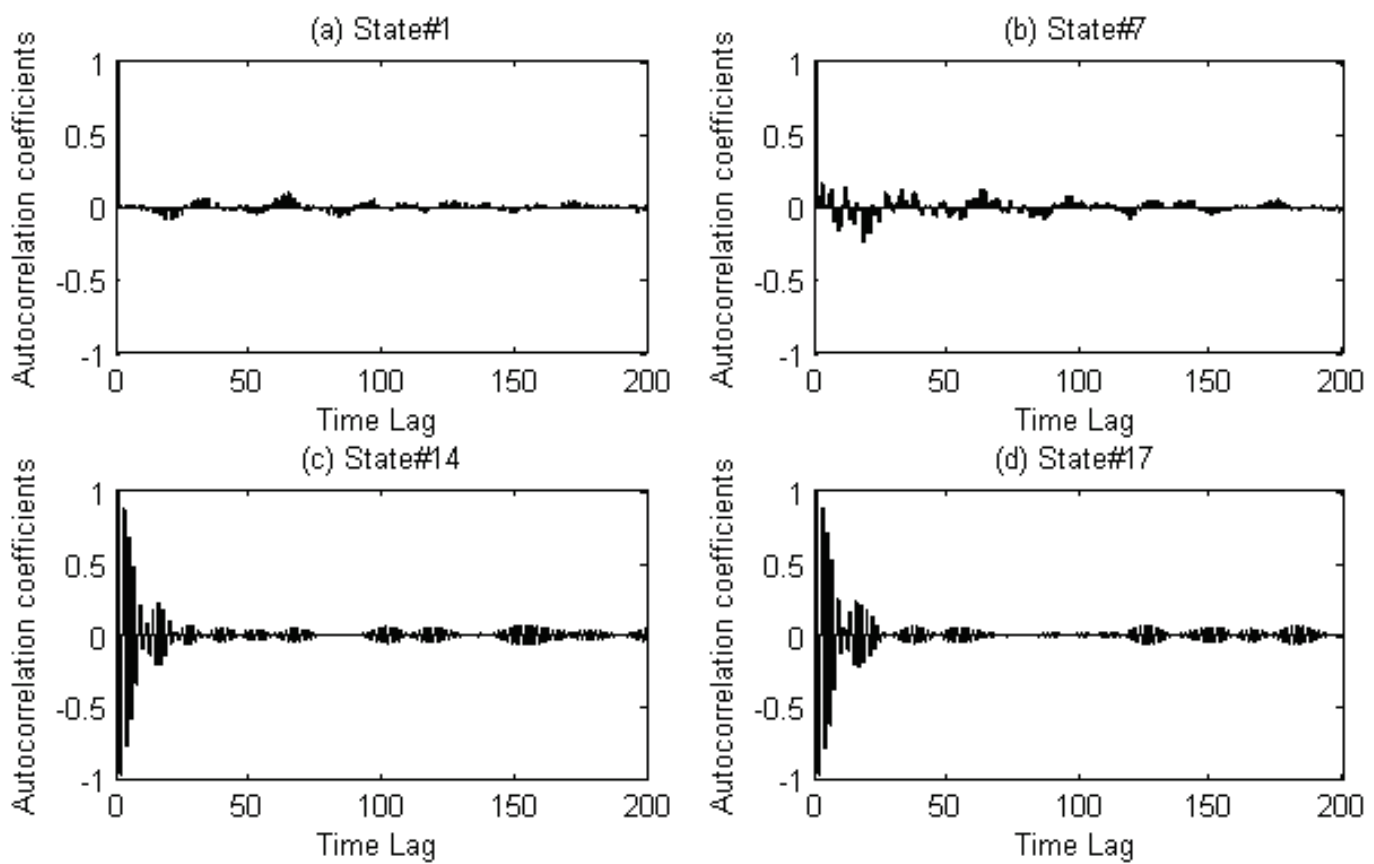

Figure 39: Autocorrelation functions of the $A R(30)$ residual errors. 
Another way to check the existence of patterns in the data is by means of the lag plot technique (NIST/SEMATECH, 2008). For completeness, a brief description of this technique is given as follows. For a time-discrete variable $X$, the lag plot consists of plotting the values of $x_{i}$ versus $X_{i-\tau}$, where $\tau$ is the lag. If the underlying data are not random, it is possible to identify a trend or pattern in the lag plot. Lag plots can be generated for any arbitrary lag; however, the most commonly used lag is 1 . Furthermore, the lag plot can be used to identify the existence of outliers. Note that a cluster around a straight line with a positive slope is an indication of positive correlation in the observations.

Figure 40 shows plots of the original acceleration-time histories from Channel 5 of States $\# 1, \# 7, \# 14$, and \#17 for a lag equal to 1 . The damage seems to introduce slight changes into the correlation among the original data. However, these changes are amplified using the residual errors from the AR(5) and AR(30) models, as shown in Figure 41 and Figure 42, respectively. The plots suggest that both undamaged states (States \#1 and \#7) are randomly distributed with no correlation. However, the nonlinearities associated with damaged states (States \#14 and \#17) introduce some autocorrelation into the AR residual errors. Note that this analysis discriminates the undamaged and damaged state conditions, even in the presence of operational and environmental variations.

In conclusion, the autocorrelation function, as well as the lag plot, suggests that although the time histories of each state condition have some correlation, damage introduces strong correlation in the AR residual errors, whereas there is no correlation in the residual errors for undamaged states, even in the presence of operational and environmental variations. This fact indicates that the AR residual errors can be efficiently used as damage-sensitive features, which confirms the previous studies in the literature.

(a) State\#1

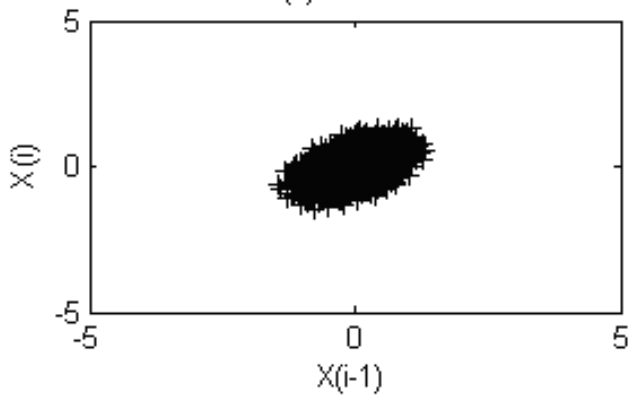

(c) State\#14

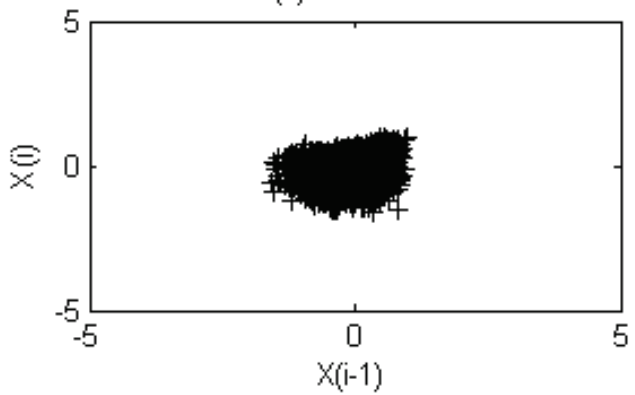

(b) State\#7

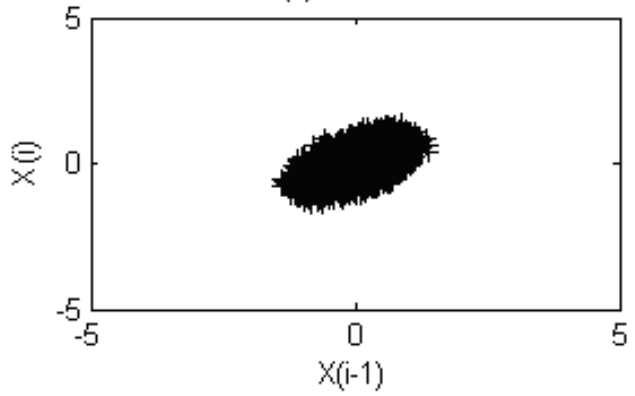

(d) State\#17

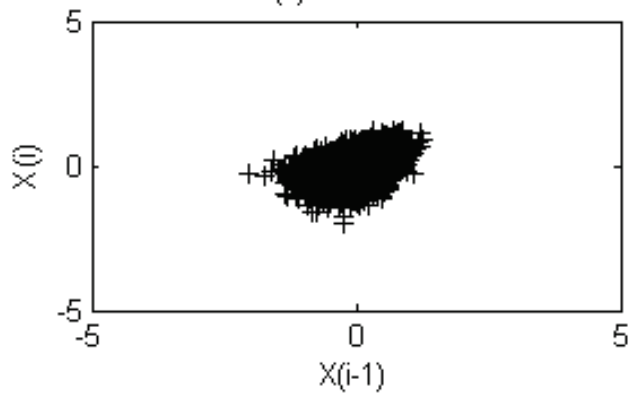

Figure 40: Lag plots of the original time history from Channel 5. 
(a) State\#1

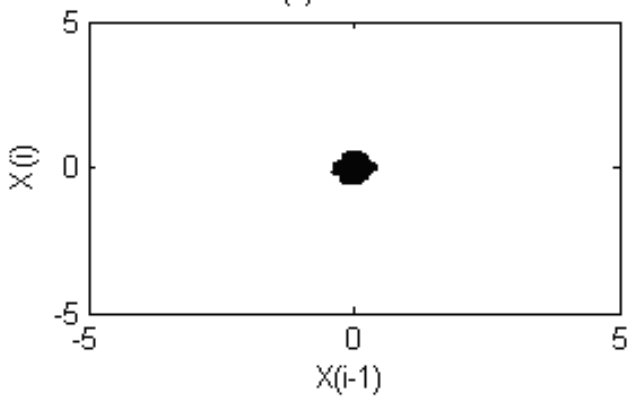

(c) State\#14

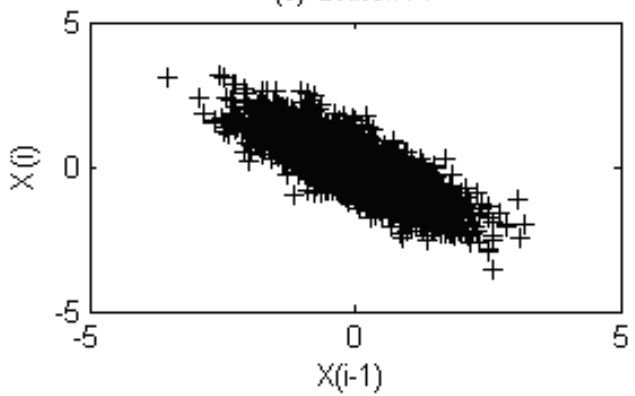

(b) State\#7

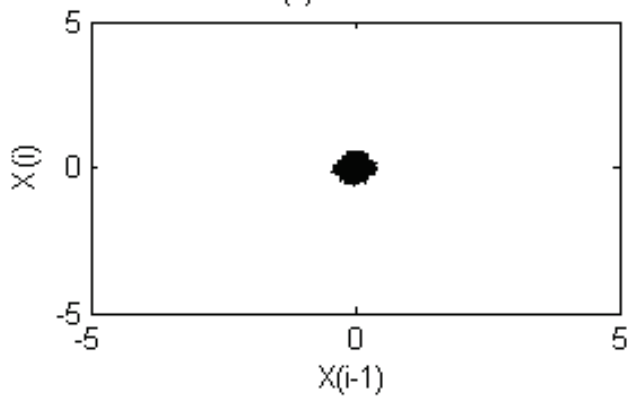

(d) State\#17

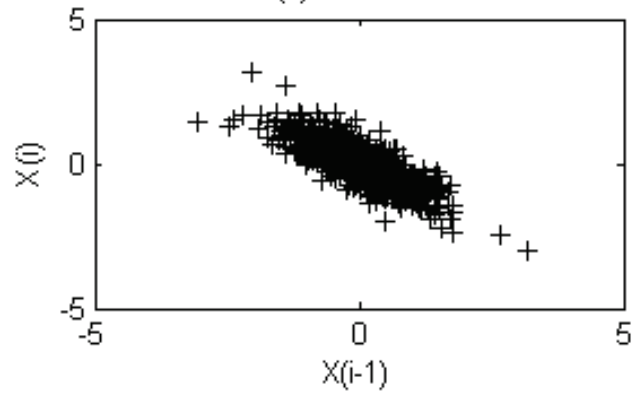

Figure 41: Lag plots of the AR(5) model residual errors from Channel 5.

(a) State\#1

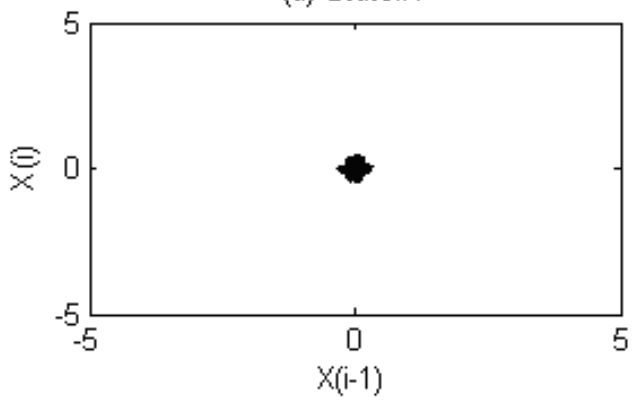

(c) State\#14

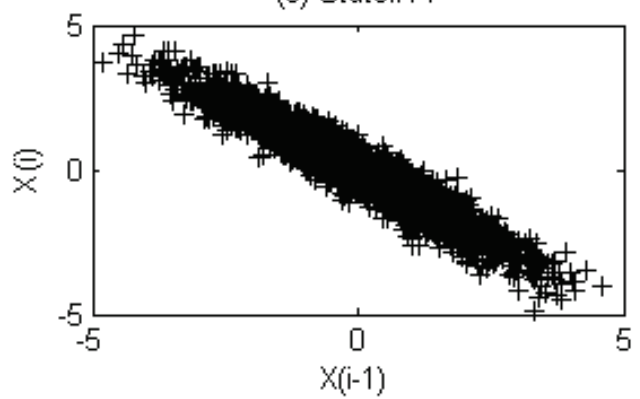

(b) State\#7

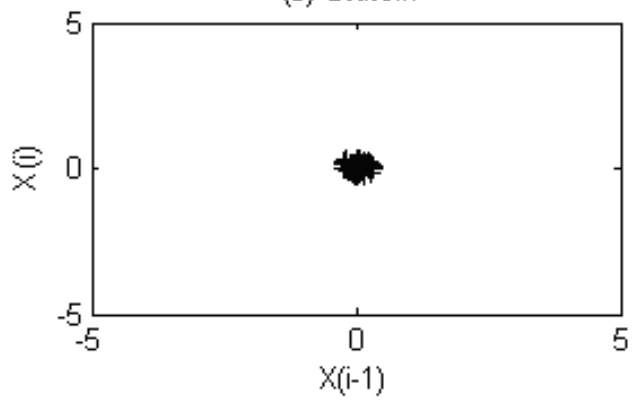

(d) State\#17

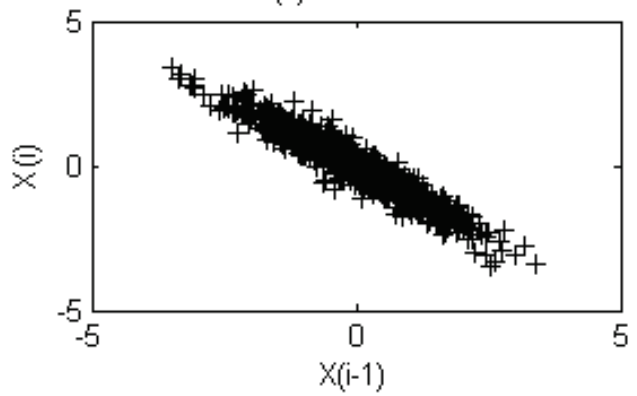

Figure 42: Lag plots of the AR(30) model residual errors from Channel 5. 


\subsubsection{Principal Component Analysis of Autoregressive Parameters}

Principal components analysis (PCA) is a classical technique of multivariate statistics for mapping multidimensional data into a lower dimension with a minimal loss of information (Bishop, 1995; Jolliffe, 2004). A brief description of this technique is given as follows.

Mathematically, the goal of PCA is to map $n$ vectors $x^{n}$ in a $d$-dimensional space $\left(x_{1}, \ldots, x_{d}\right)$ onto $n$ vectors $z^{n}$ in a $p$-dimensional space $\left(z_{1}, \ldots, z_{p}\right)$, where $p<d$. Basically, the vectors $x^{n}$ can be represented, without loss of information, as a linear combination of a set of $d$ orthogonal vectors $u_{i}$

$$
x^{n}=\sum_{i=1}^{d} z_{i}^{n} u_{i}
$$

This technique performs an orthogonal transformation by retaining only the significant eigenvectors. The eigenvectors with the higher magnitude of eigenvalues are the principal components of the data set and they correspond to the dimensions that have the largest variability in the data set. More precisely, for a $d$-dimensional data set with $n$ vectors, it is possible to calculate $d$ eigenvectors, and then, choosing only the first $p$ eigenvectors, the final data set can be rewritten with only $p$ dimension $(p<d)$ without significant loss of information. The $u_{i}$ satisfy the following system of equations

$$
\sum u_{i}=\lambda_{i} u_{i}
$$

where $\sum$ is the covariance matrix of the set of $n$ vectors and $\lambda_{i}$ are the eigenvalues.

The principal components can also be obtained using SVD technique by decomposing $\Sigma$ in the form of

$$
\sum=V \Lambda V^{T}
$$

where $\Lambda$ is a diagonal matrix containing the ranked eigenvalues $\lambda_{i}$, and $V$ is the matrix containing the corresponding eigenvectors $u_{i}$. Notice that the results of PCA are generally discussed in terms of components scores $\left(z_{i}\right)$ and loadings $\left(u_{i}\right)$.

In the SHM field, PCA is used for three primary purposes: (i) evaluation of patterns in the data; (ii) data cleansing; and (iii) data compression. The evaluation of patterns in the data is achieved through a linear mapping of data from the original feature space into a transformed feature space, where the eigenvectors (principal components) are orthogonal to each other and, as a consequence, they are uncorrelated. One can extract lines or patterns that characterize the data. The eigenvalues provide information about the variance of the principal components. The eigenvectors with the higher magnitude of eigenvalues are the principal components of the data. Data cleansing is a process used to discard those linear combinations of the data that have small contributions to the overall variance, i.e., the principal components with lower eigenvalues. This process can be achieved by reversing the projection back to the original feature space using only the principal components with higher eigenvalues. Data compression is the process of reducing the dimensionality of the data or the feature. Data compression can be achieved by reversing the projection back to the original feature space using only the principal components with higher eigenvalues. However, mapping the data into a lower dimension space may result in a loss of some information that can be necessary to discriminate the damaged from undamaged states. 
In this study, PCA technique is applied to evaluate patterns in the data for each state condition. Figure 43 illustrates the variability explained by each principal component for two extreme state conditions: baseline condition (State \#1) and the most damaged condition (State \#14). In this case the number of vectors is equal to ten, i.e., equal to the number of time histories for each state condition from Channel 5. The plots show that both states are characterized by a well-defined pattern because the first principal component explained almost $100 \%$ of the variability.

In order to investigate the use of AR parameters as damage-sensitive features in a transformed feature space created using the PCA technique, Figure 44 displays the AR(30) parameters from Channel 5 for all the state conditions projected onto the first two principal components. The state conditions are divided into four groups as stated in Subsection 2.3: baseline condition (BC); undamaged conditions with operational and environmental effects (UC); damaged condition (DC); and damaged condition with operational and environmental effects (DCOEE). The visualization of the AR parameters in the transformed space shows three principal characteristics: (i) the undamaged and damaged state conditions are not linearly separable in a two-dimensional projection; (ii) in general, the parameters of undamaged states seem to populate the borders of an ellipse; and (iii) the parameters from the undamaged state conditions have higher variance in the first principal component than the ones from the damaged state conditions as shown in Figure 45.

Notice that the projection assumes the same shape by projecting the first principal component onto the remaining principal components because all of those components have residual variance as shown in Figure 43. In conclusion, using the PCA of the AR parameters to project these parameters onto the first two principal components does not result in a linear separation of the state conditions. PCA does show that the undamaged and damaged state conditions cluster well. However, the damaged cluster falls within the undamaged cluster, implying that some projection techniques such as support vector machines are needed to define a damaged-undamaged linear decision boundary in a higher dimensional space.

(a) State\#1

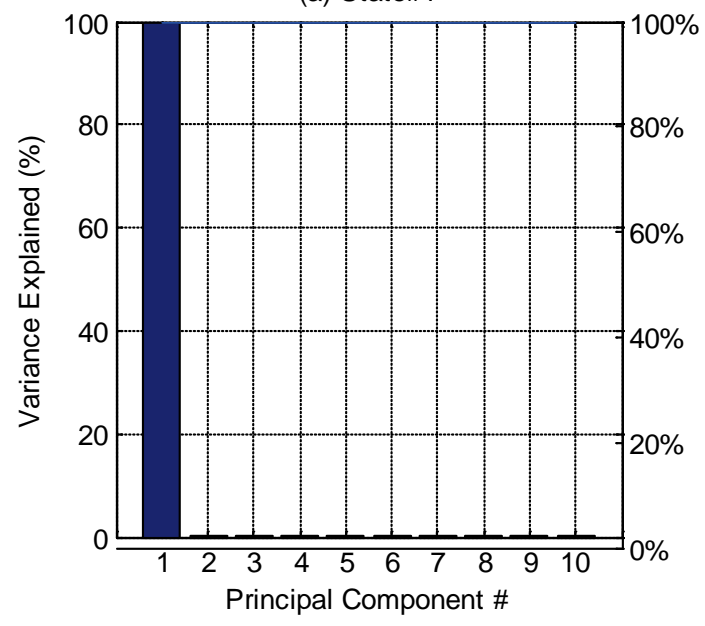

(b) State\#14

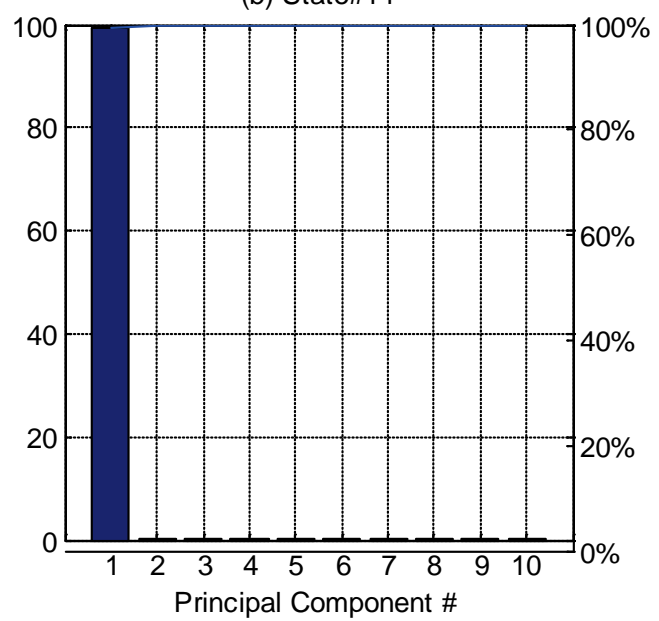

Figure 43: $\quad$ Plot of the percentage variability explained by each principal component (Channel 5). 


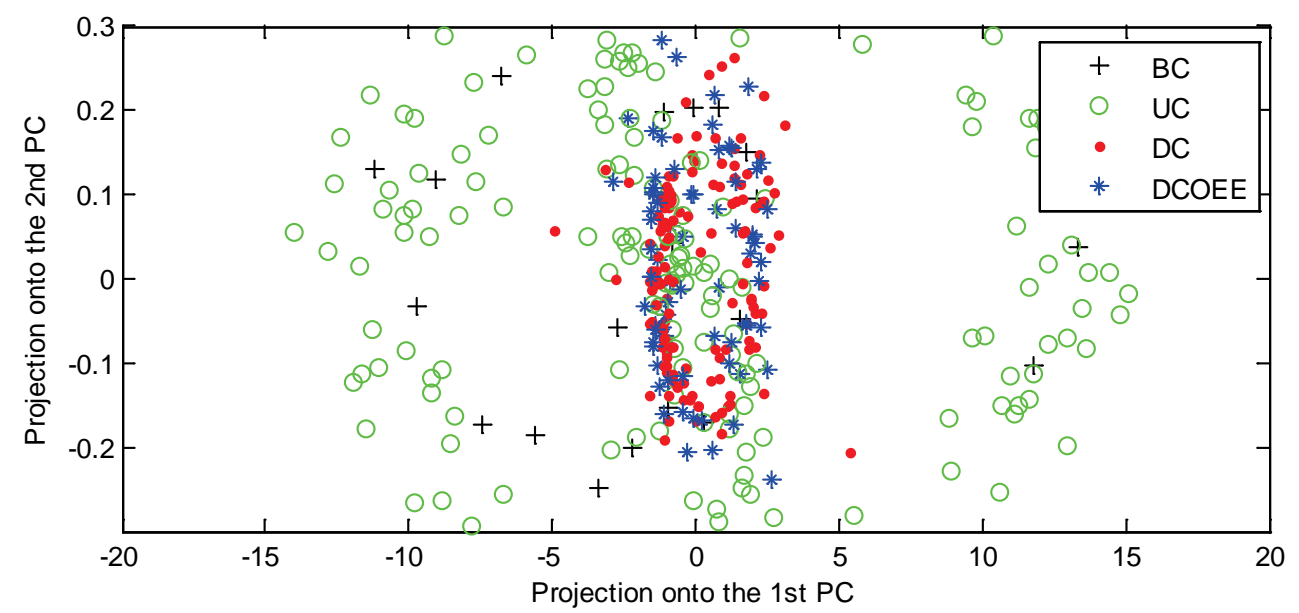

Figure 44: $A R(30)$ parameters (Channel 5) projected onto the first two principal components.

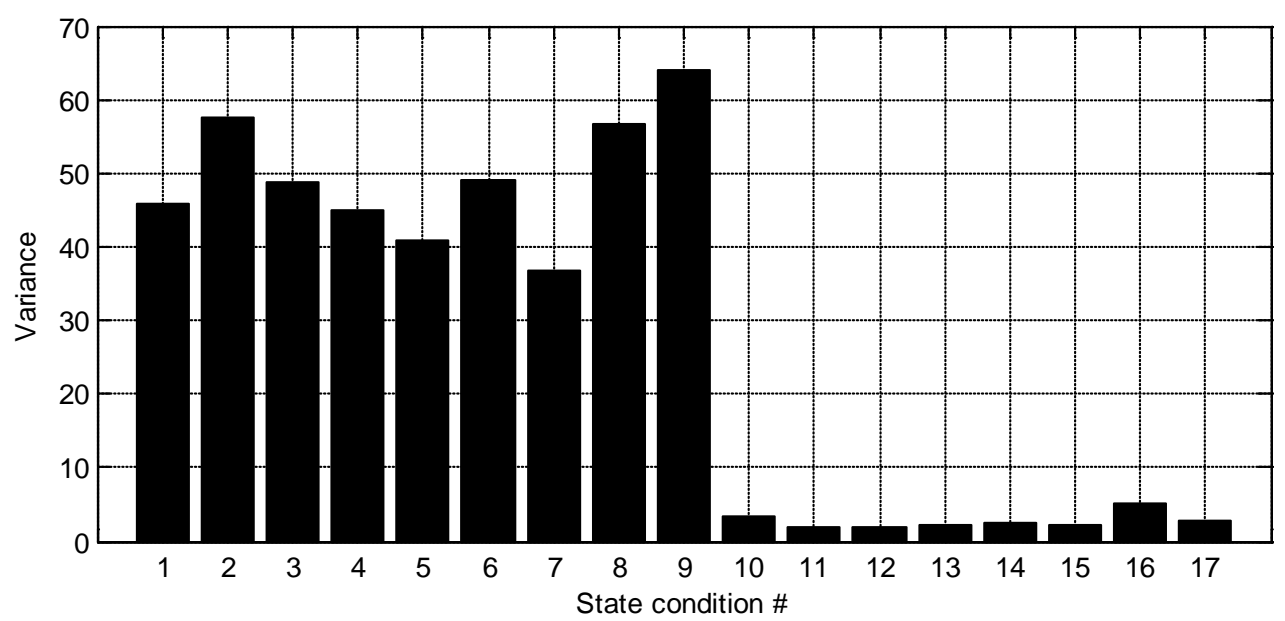

Figure 45: $\quad$ Plot of the variability explained by the first principal component of each state (Channel 5).

\subsubsection{Correlation Coefficient Using Time Domain Data}

This subsection uses the correlation coefficient between times histories to find correlations among the structural state conditions. For completeness, a brief definition of the correlation coefficient is given below.

The covariance matrix, $\sum$, describes the correlation between elements of a vector or elements of a matrix. It is a generalization of the concept of the variance of a scalar-valued random variable to higher dimensions. For $N$ variables, the covariance matrix has a dimension of $(N \times N)$. Considering two random variables $X$ and $Y$, the covariance between those variables is given by

$$
\operatorname{cov}(X, Y)=E\left[\left(X-\mu_{X}\right)\left(Y-\mu_{Y}\right)\right],
$$

where $E$ is the mathematical expectation, and $\mu_{X}$ and $\mu_{Y}$ are the means of each variable. Then, the correlation coefficient is given by 


$$
\rho_{X Y}=\frac{\operatorname{cov}(X, Y)}{\sigma_{X} \sigma_{Y}},
$$

where $\sigma_{X}$ and $\sigma_{Y}$ are the standard deviations of each variable. Therefore, the correlation can be interpreted as a standardized covariance. Note that the correlation coefficient is a zero-lag normalized correlation function given by Eq. (7). The unbiased estimator for the correlation coefficient between $X$ and $Y$ based on two samples of dimension $n$, sample standard deviations $S_{X}$ and $S_{y}$, and samples means of $\bar{X}$ and $\bar{y}$, is given by

$$
r_{x y}=\frac{1}{(n-1)} \frac{\sum_{i=1}^{n}\left(x_{i}-\bar{x}\right)\left(y_{i}-\bar{y}\right)}{s_{x} s_{y}} .
$$

In order to investigate the use of correlation coefficients as potential damage-sensitive features to identify the existence and locate the damage among the sensors, a standardized covariance matrix $\sum$ with elements defined by Eq. (33) was calculated for each state condition. In this case, the variables are one time history from each channel (2 to 5). Because the accelerometers are distributed at four different locations, each matrix $\sum$ has a dimension of $(4 \times 4)$.

Figure 46 shows three coefficients per state condition, namely $r_{25}, r_{35}$, and $r_{45}$. Notice that the indices of the coefficients correspond to the channel's number instead of the position in the matrix. For instance, $r_{25}$ consists of the correlation coefficient between Channel 2 and 5 . The correlation coefficients based on the time histories are not able to discriminate the damaged from undamaged states or to locate the damage among the sensors, because the coefficients follow no systematic pattern that can be associated with the damaged states. This fact can be justified by considering the singularities imposed by the bumper (see Subsection 4.4.3). The singularities manifest themselves as a local phenomenon in time, which is not enough to produce trends in the time histories that can be identified by a global parameter such as the correlation coefficient. 
(a) Correlation coefficient - r25

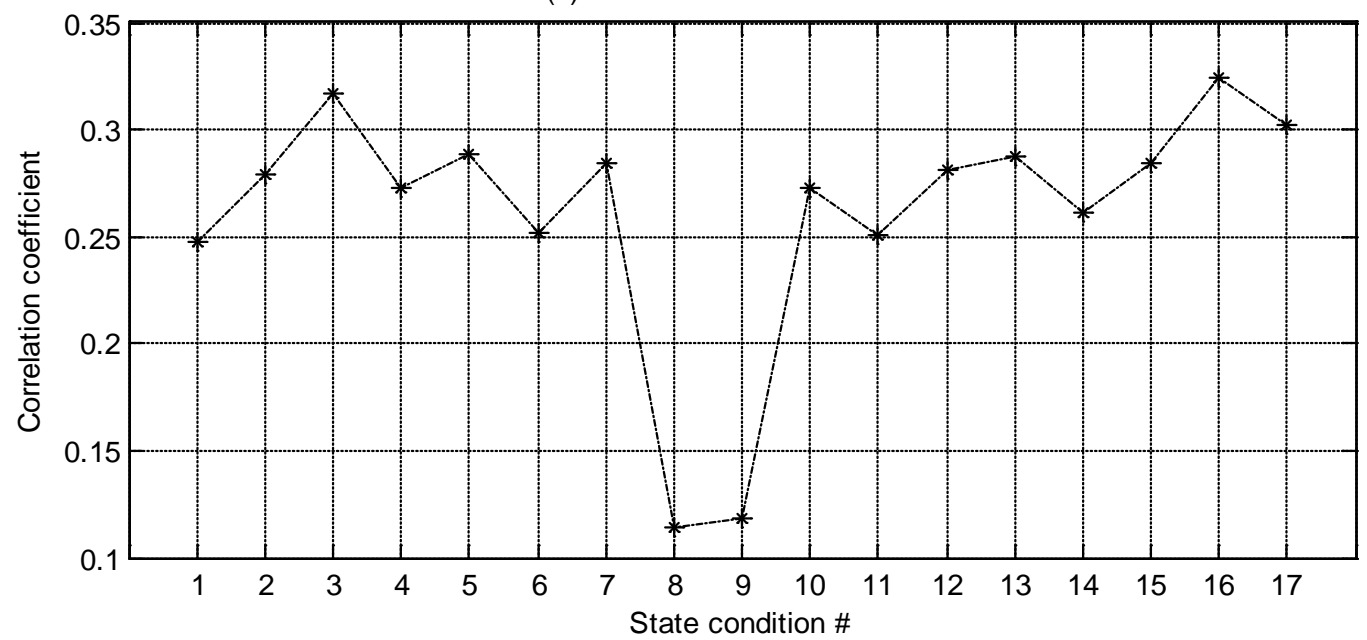

(b) Correlation coefficient - r35

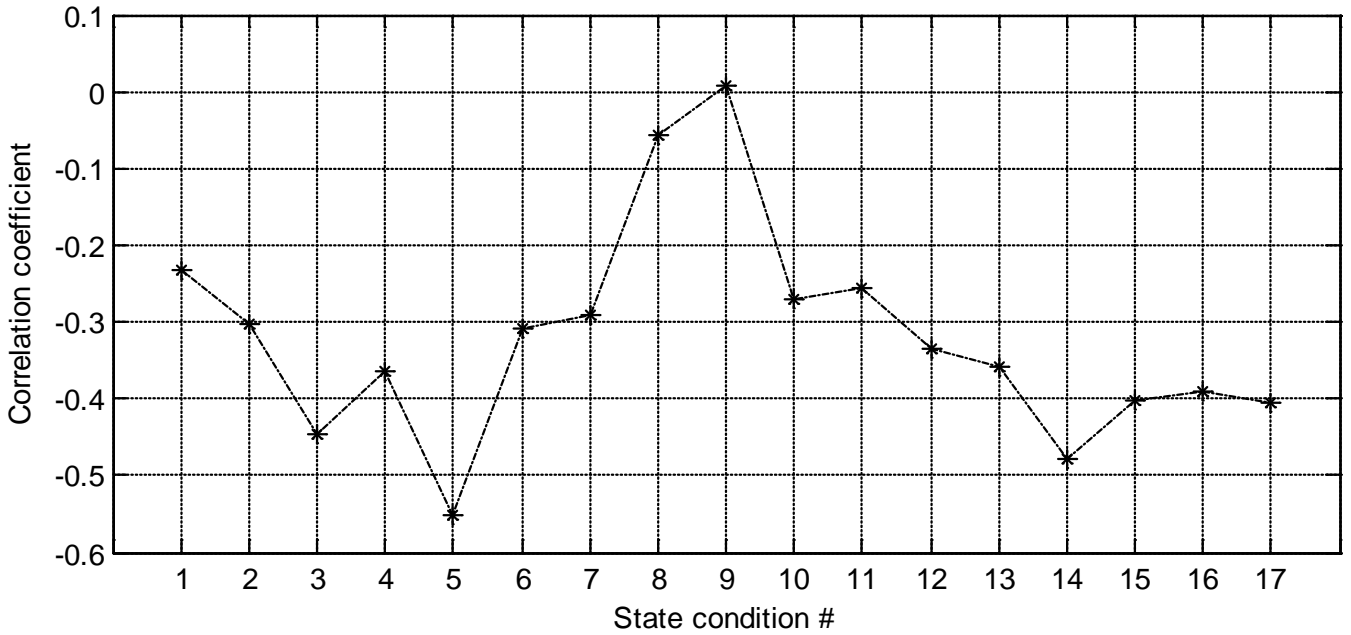

(c) Correlation coefficient - r45

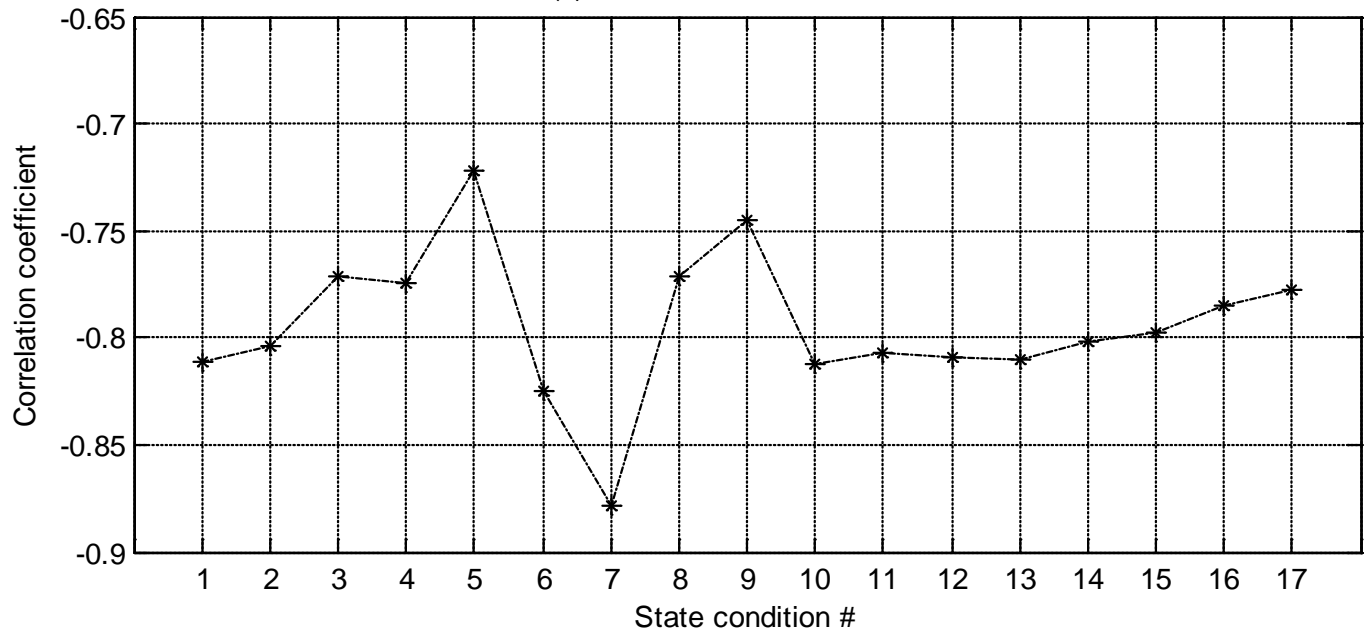

Figure 46: Correlation coefficient, $r_{i j}$, by state condition, between Channels $i$ and $j$. 


\subsection{Frequency Domain Analysis}

The analysis carried out in this subsection intends to highlight the limitations of using modal parameters as damage-sensitive features, specifically the natural frequencies. Additionally, correlation analyses are performed using FRFs.

\subsubsection{Modal Parameters}

Modal parameters as damage-sensitive features were widely used in early SHM studies (Doebling et al., 1996). The motivation behind this approach is that modal parameters (natural frequencies, mode shapes, and modal damping) are functions of the physical properties of the structure (mass, damping, and stiffness). Therefore, any changes in the physical properties caused by structural damage will result in changes in the modal parameters. However, many studies using this approach only investigate numerical models or simple laboratory structures, and, therefore, they do not include operational and environmental variability and their associated effects on modal parameters. This variability can cause changes in the modal parameters that can mask the changes resulting from damage. Furthermore, it has been found in many cases that modal parameters do not have the required sensitivity to small defects in a structure (Farrar et al., 2000). Thus, in this subsection some limitations associated with the use of modal parameters as damage-sensitive features will be discussed.

The modal parameters estimation is done by curve fitting a parametric form of the FRF, defined in terms of the resonant frequencies, modal damping, and mode shapes, to the measured FRFs from one test corresponding to each state condition. The frequencies, damping ratios, and modes shapes were estimated using an RFP global curve fitting method using commercial modal analysis software. The RFP is a frequency-domain curve-fitting method that operates directly on the complex FRF. This method performs a least-squared error-curve fit to all FRFs. Note that the curve fitting is applied to minimize the effects of measurement noise.

The specified frequency bands over which the RFP method was applied were determined by visualizing the imaginary part of the complex mode indicator function (CMIF), as shown in Figure 47 for State \#1. By looking at this plot, three frequencies can be clearly identified. Therefore, for all state conditions, the FRFs were curve fit in one window with a frequency band between 25 and $80 \mathrm{~Hz}$, as shown in Figure 48. Table 4 summarizes the estimated natural frequencies and damping ratios corresponding to each state condition. For completeness, the mode shapes of the baseline condition are plotted in Section 3.

In order to highlight the differences from the baseline condition (State \#1), Figure 49 shows the natural frequencies of all state conditions and Figure 50 plots the frequency differences between the baseline condition and the other states. One can observe that in general the differences decrease for the undamaged state conditions (States \#2-\#9) and increase for the damaged states with no mass or stiffness changes (States \#10-\#14). However, the challenge of this study is to detect damage when the structure is also affected by operational and environmental changes. Thus, as shown in Figure 50, it is clear that changes in frequencies associated with the damage introduced for States \#15, \#16, and \#17 are masked by the varying mass and stiffness values associated with these state conditions.

Note that apparently the damping ratios do not show signs of consistently increasing or decreasing with the damaged state conditions. Theoretically, the impacts associated with damage should increase the energy dissipation, which should manifest itself in higher damping. However, damping ratios are empirical values that must be obtained by measurements that assume a linear system. As a consequence, the lack of consistent changes in damping as a 
function of damage can be justified by the fact that the damping ratios are estimated through the FRF curve-fitting process that fits a linear modal model to these nonlinear-system response data. Another aspect that can lead to discrepancies in the damping estimate is the use of the Hanning window to estimate the FRFs, which tends to bias the damping ratio estimates and yields values higher than are actually present (Maia et al., 1997).

In conclusion, this subsection has demonstrated some limitations of using modal parameters as damage-sensitive features, specifically the natural frequencies. One cannot conclude which data are from the damaged states by just examining changes in the natural frequencies when other sources of variability are present that influence the dynamic response characteristics of the structure.

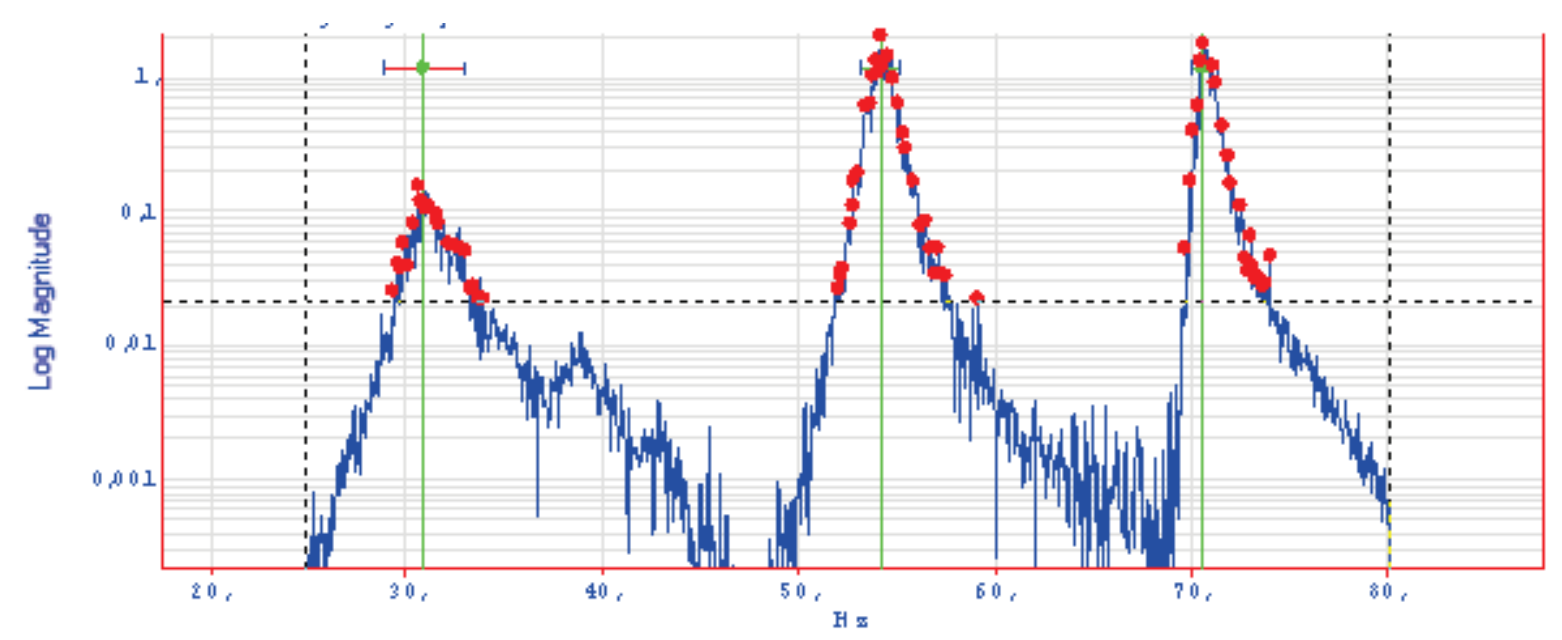

Figure 47: CMIF for one FRF from Channel 5 of State \#1.

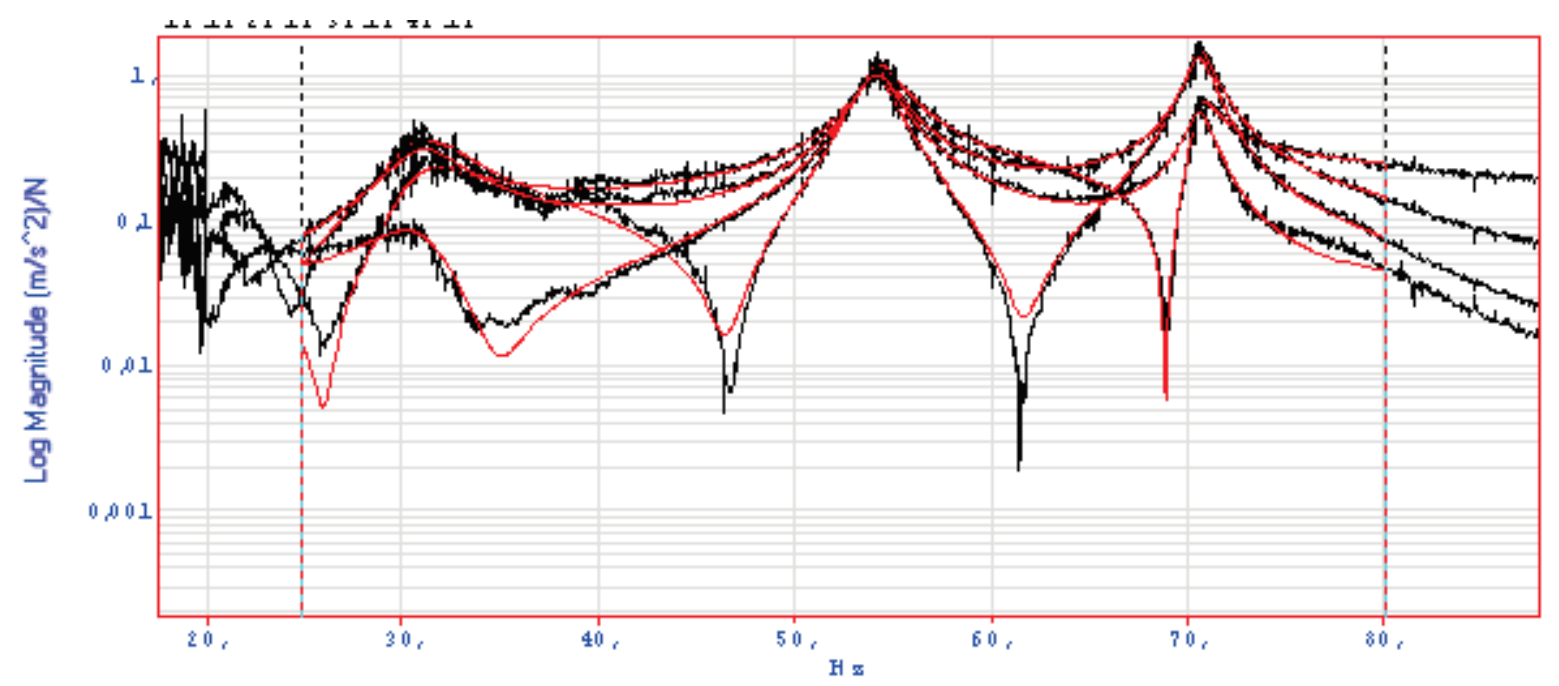

Figure 48: Curve fitting example for the FRFs from Channels 2-5 of State \#1. 
Table 4: Experimental Natural Frequencies and Damping Ratios of All State Conditions

\begin{tabular}{|l|l|l|l|l|l|l|}
\hline \multirow{2}{*}{ State condition } & \multicolumn{3}{|c|}{ Frequency (Hz) } & \multicolumn{3}{c|}{ Damping ratio (\%) } \\
\cline { 2 - 7 } & 2nd & 3rd & 4th & 2nd & 3rd & 4th \\
\hline State \#1 & 30.7 & 54.2 & 70.7 & 6.3 & 2.0 & 0.97 \\
\hline State \#2 & 30.4 & 52.9 & 70.3 & 6.4 & 1.5 & 0.76 \\
\hline State \#3 & 30.9 & 53.1 & 68.2 & 5.5 & 2.1 & 0.82 \\
\hline State \#4 & 30.9 & 51.2 & 69.2 & 7.1 & 2.2 & 0.55 \\
\hline State \#5 & 30.3 & 47.0 & 67.8 & 7.0 & 1.8 & 0.38 \\
\hline State \#6 & 29.7 & 53.9 & 65.8 & 5.3 & 1.7 & 1.2 \\
\hline State \#7 & 28.6 & 54.2 & 62.2 & 5.1 & 1.7 & 0.72 \\
\hline State \#8 & 30.2 & 51.1 & 69.3 & 5.6 & 2.2 & 0.80 \\
\hline State \#9 & 28.9 & 47.4 & 68.0 & 4.6 & 2.6 & 0.80 \\
\hline State \#10 & 31.1 & 54.4 & 70.9 & 6.6 & 2.1 & 1.0 \\
\hline State \#11 & 31.7 & 54.5 & 70.9 & 7.0 & 1.9 & 0.93 \\
\hline State \#12 & 31.8 & 54.9 & 71.2 & 6.3 & 1.9 & 1.0 \\
\hline State \#13 & 32.4 & 55.2 & 71.4 & 6.3 & 1.9 & 1.0 \\
\hline State \#14 & 33.5 & 57.6 & 74.2 & 7.1 & 2.2 & 0.97 \\
\hline State \#15 & 31.6 & 54.0 & 71.1 & 5.4 & 1.6 & 0.73 \\
\hline State \#16 & 31.0 & 53.4 & 68.3 & 5.3 & 2.3 & 0.82 \\
\hline State \#17 & 32.3 & 54.4 & 69.2 & 5.0 & 2.2 & 0.80 \\
\hline
\end{tabular}

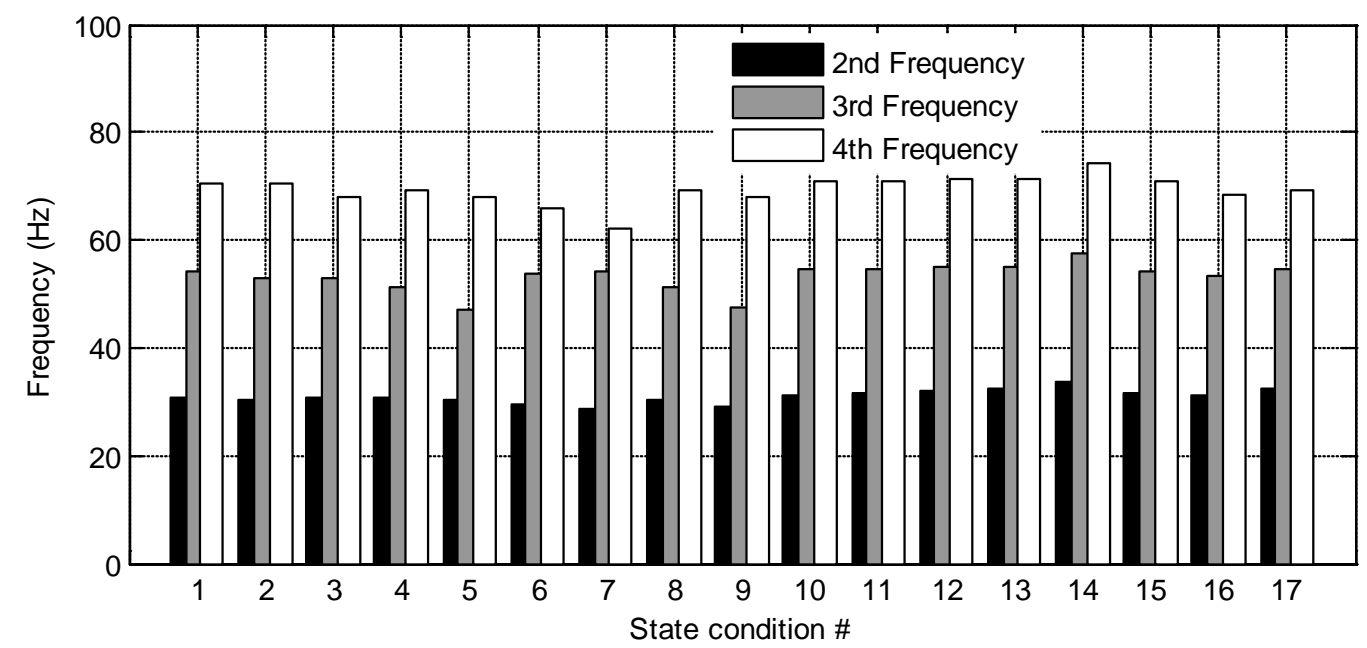

Figure 49: Natural frequencies for all state conditions. 


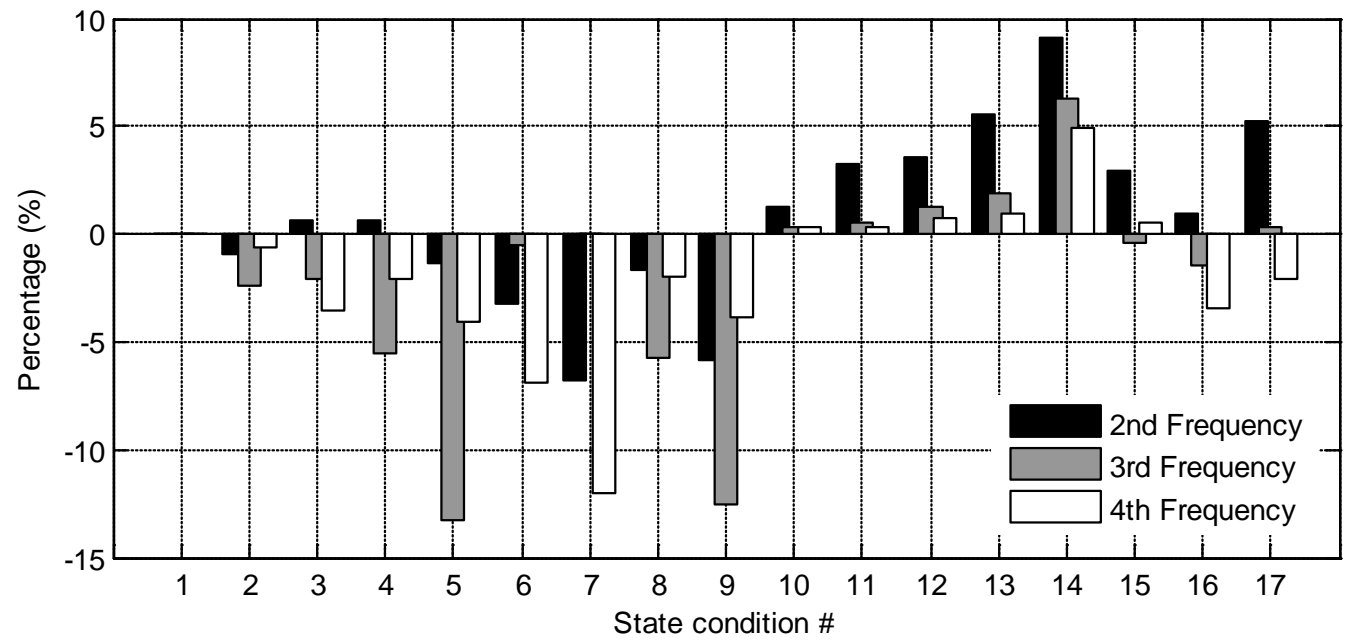

Figure 50: Frequency deviations from the baseline condition (State \#1) for all state conditions.

\subsubsection{Correlation Coefficients Using Frequency Response Functions}

In Subsection 4.2.3, the correlation coefficient was used to develop a global measure of the correlation between two time histories. Furthermore, time-domain data was used to investigate the usefulness of correlation coefficients across the sensors as potential damagesensitive features. Now, the same procedure is carried out using frequency domain data, namely the FRFs. Therefore, the variables of each standardized covariance matrix, $\sum$, composed of correlation coefficients defined in Eq.(33), are the FRFs of each channel (2 to 5) and, therefore, for each state condition, the matrix $\sum$ has a dimension of $(4 \times 4)$.

Figure 51 shows three correlation coefficients $r_{25}, r_{35}$, and $r_{45}$ per state condition. As with the time-domain data, the results show that the correlation coefficients estimated from the FRFs do not show any describable deterministic pattern across the sensors to discriminate the undamaged and damaged state conditions.

Instead of using the correlation coefficient to analyze the correlation across the sensors for each state condition, the correlation coefficient can also be used to analyze the correlation between FRFs corresponding to different states. In this approach, the matrix $\sum$ is formed for each channel. Because there are seventeen state conditions, for each channel the matrix $\sum$ has a dimension of $(17 \times 17)$. Figure 52 (a) and (b) show the correlation coefficients between the baseline (State \#1) and all the structural state conditions (States \#1-\#17) at Channels 4 and 5, respectively. Once again, these figures show that the use of correlation of the FRFs does not discriminate the undamaged and the damaged state conditions.

In conclusion, the correlation coefficient analysis was carried out to see if this feature can be used to identify the existence of damage as well as to locate the damage across the sensors. For both cases, either looking at changes in FRFs across the sensors for a particular damage case or looking at changes between an FRF of the baseline and all states conditions, the correlation coefficients do not discriminate the undamaged and damaged state conditions. This fact may be associated with the operational and environmental variations that introduce changes in the frequency content of the FRF and, therefore, those variations mask the changes in correlation associated with the damaged state conditions. 
(a) Correlation coefficient - r25

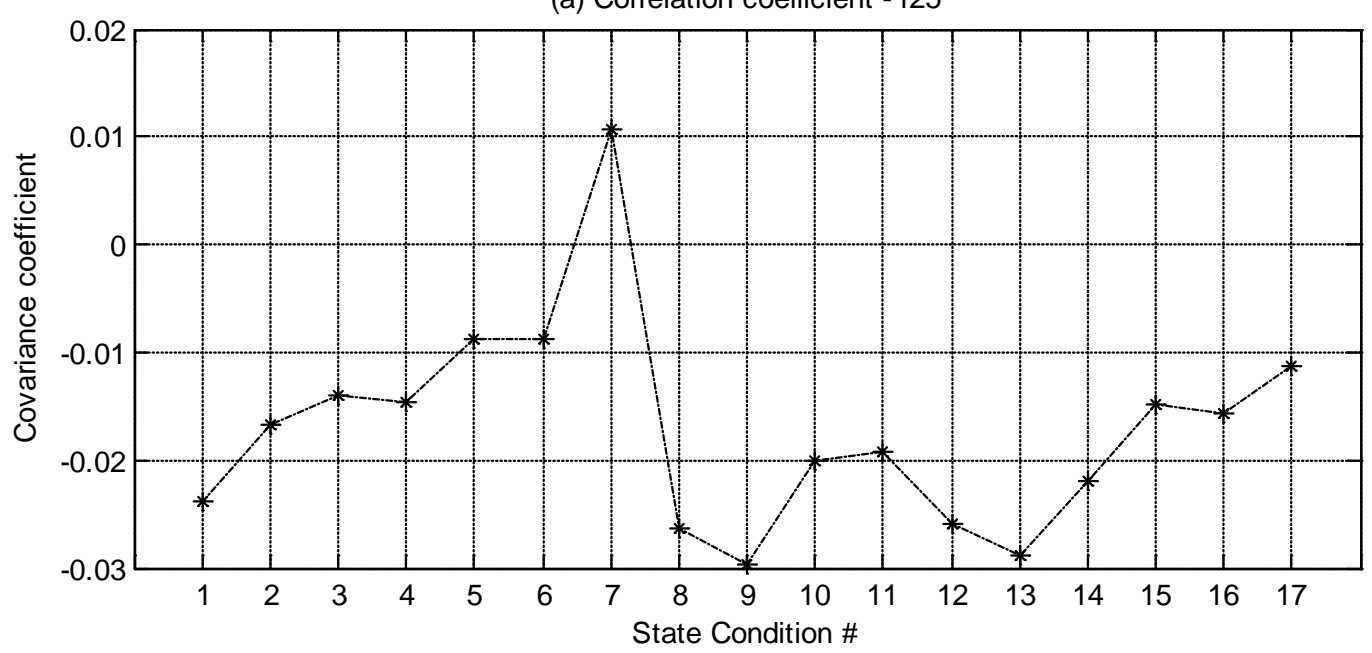

(b) Correlation coefficient - r35

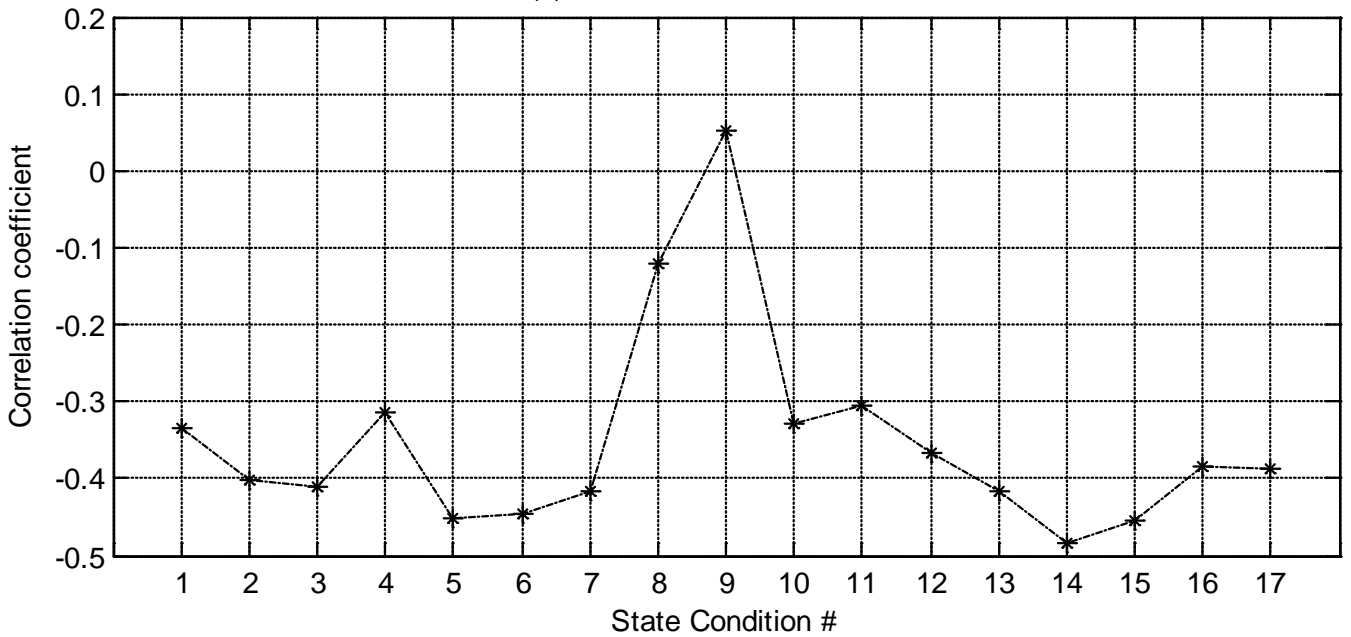

(c) Correlation coefficient - r45

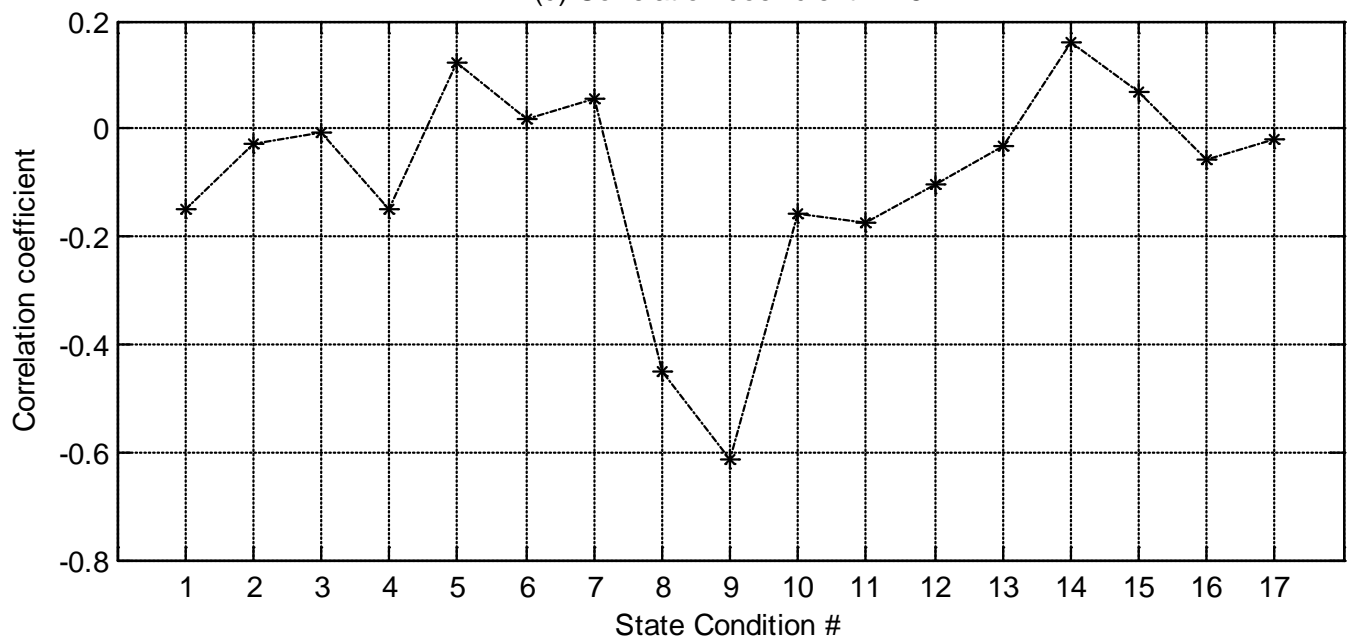

Figure 51: Correlation coefficient per state condition across the channels. 
(a) Correlation coefficient - Channel 4

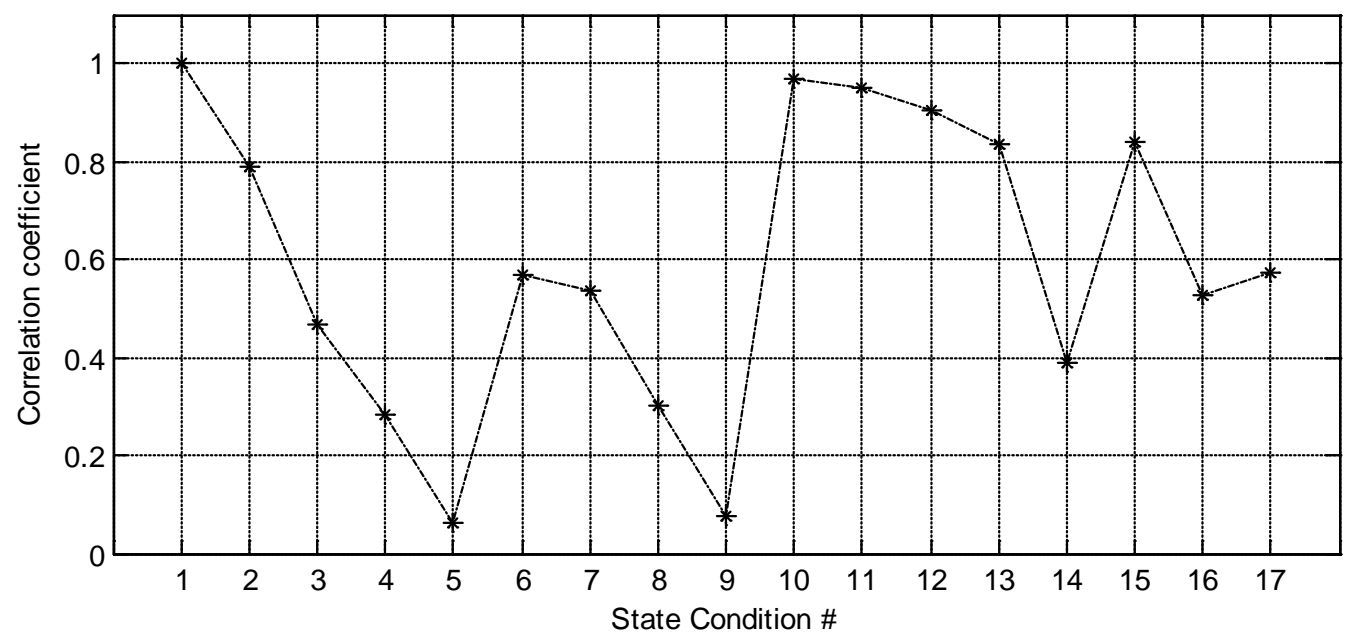

(b) Correlation coefficient - Channel 5

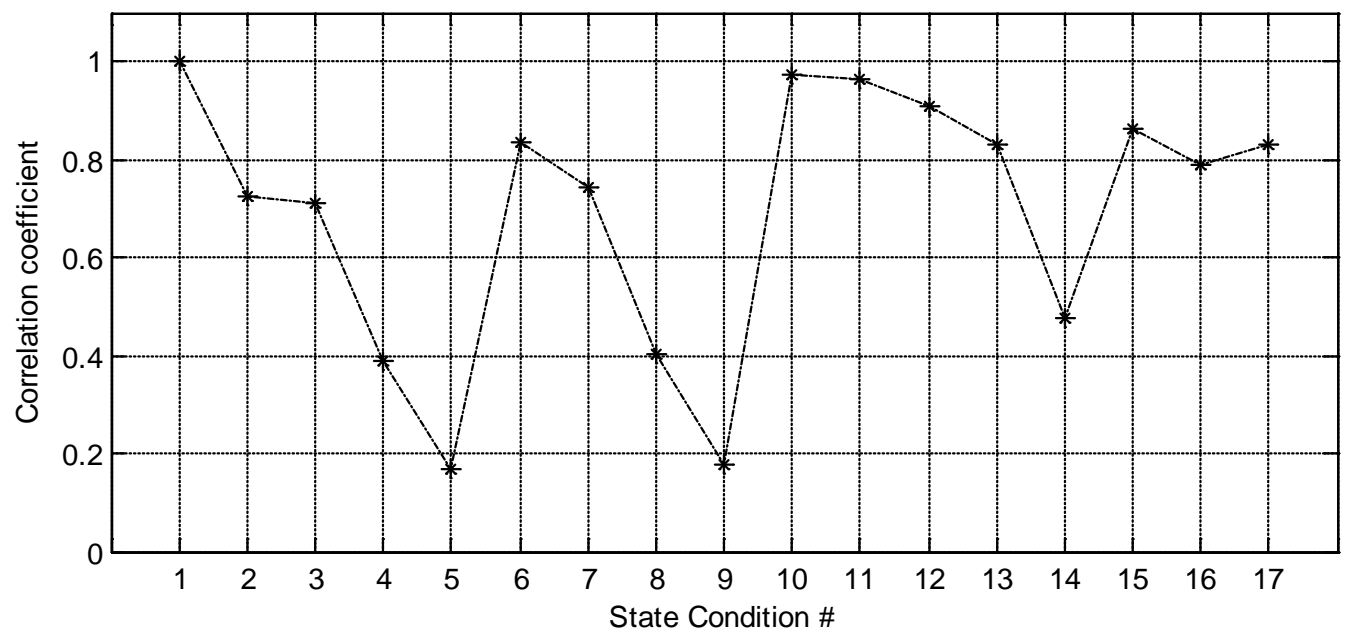

Figure 52: Correlation coefficient per state condition.

\subsection{Time-Frequency Analysis}

This subsection examines the time-frequency-amplitude representations of the signals in an effort to identify the damage. The main advantage of this representation is to track the evolution of the frequency components of the signal over time.

For a stationary system, the frequency content should not change over time. However, nonlinearities introduced into the structure by a bumper can result in a nonstationary system. Therefore, if damage manifests itself as a nonlinearity, the signals from a damaged structure can be time-variant and, as a consequence, the frequency content may change with respect to time in a manner that can be correlated with damage.

\subsubsection{Short-Time Fourier Transform}

The STFT is used to determine the frequency content of small segments of a signal over time. The Fourier transform algorithm is applied to a subset or window of the complete time history and is used to calculate related spectral quantities for this window of data such as the PSD. This process is repeated using a moving window, while the data windows are allowed to overlap. This technique maps a signal into a function of both the time and frequency domains. 
The STFT provides information about the frequency content and also about how the frequency content evolves over time. For a given sampling rate, the frequency resolution of the STFT is determined by the time length or period of the window. Notice that for a signal from a stationary system, the frequency content should not change over time. The spectrogram command in Matlab is used to compute the STFT and to visualize how the frequency components change over time.

In this study, each 8,192-point acceleration-time history is split into 512-point time windows with a $50 \%$ overlap in order to compute the STFT. A Hamming window is applied in each time-domain segment. For each segment, the discrete-time Fourier transform is computed to produce an estimate of the time-varying frequency content of the signal. If the time history used to compute the STFT is representative of a stationary system, it is expected that one will observe no changes in the frequency content as a function of time.

Figure 53, Figure 54, and Figure 55 illustrate the spectrogram for one time history from Channel 4 corresponding to State \#1 (baseline), State \#10 (gap $=0.20 \mathrm{~mm}$ ) and State \#14 (gap $=0.05 \mathrm{~mm}$ ), respectively. Note that in each figure the spectrogram is plotted in a timefrequency-amplitude and time-frequency representation. Figure 56 plots the concatenated format of the spectrograms in each state, where the time domain between 0-25.6 s corresponds to the State \#1, between 25.6-51.2 s to State \#10, and 51.2-76.8 s to State \#14. A few observations can be made, based on these spectrograms:

i. All three states show significant energy content around the three identified resonant frequency components, at 30.7, 54.2, and $70.7 \mathrm{~Hz}$ (for the baseline condition), which correspond very well to the results from the analytical (Section 3) and experimental modal analysis results (Section 4). Note that the first frequency component is below $20 \mathrm{~Hz}$, and it is not represented as explained in Section 2;

ii. The second resonant frequency component $(30.7 \mathrm{~Hz})$ has lower energy content than the other two;

iii. The damaged State \#14 seems to distribute energy content in more broadly across the spectra by decreasing the energy at the frequency components;

iv. There is no significant indication that the damaged States \#10 and \#14 come from a nonstationary system; however, in the case of State \#14, the damage seems to impose a steady increase in the natural frequencies over time when compared to the other two states.

Even though, theoretically, the stiffness of the structure should change throughout the measurement as a result of the impacts between the bumper and suspended column, the spectrograms do not show this result. Two reasons are presented to explain this result:

i. For a low level of damage (State \#10), the impacts occur relatively infrequently in time and are not sufficient to change the frequency components for that specific length of the window. However, for a high level of damage (State \#14), the high number of impacts makes the structure stiffer, but because many impacts occur within a given time window their effects tend to be averaged out in the spectral estimation process, and these effects are similar in every window; therefore, the system still exhibits stationary characteristics;

ii. The tradeoff between frequency resolution and the length of the window to compute the Fourier transform can be a limitation to identify changes because of high-frequency components for short durations; the lower the length of the window in time, the poorer the frequency resolution. Recall that shorter-duration windows permit good time 
resolution but poor frequency resolution. On the other hand, longer-duration windows permit good frequency resolution but have poor time resolution.

(a) Time-frequency-amplitude representation

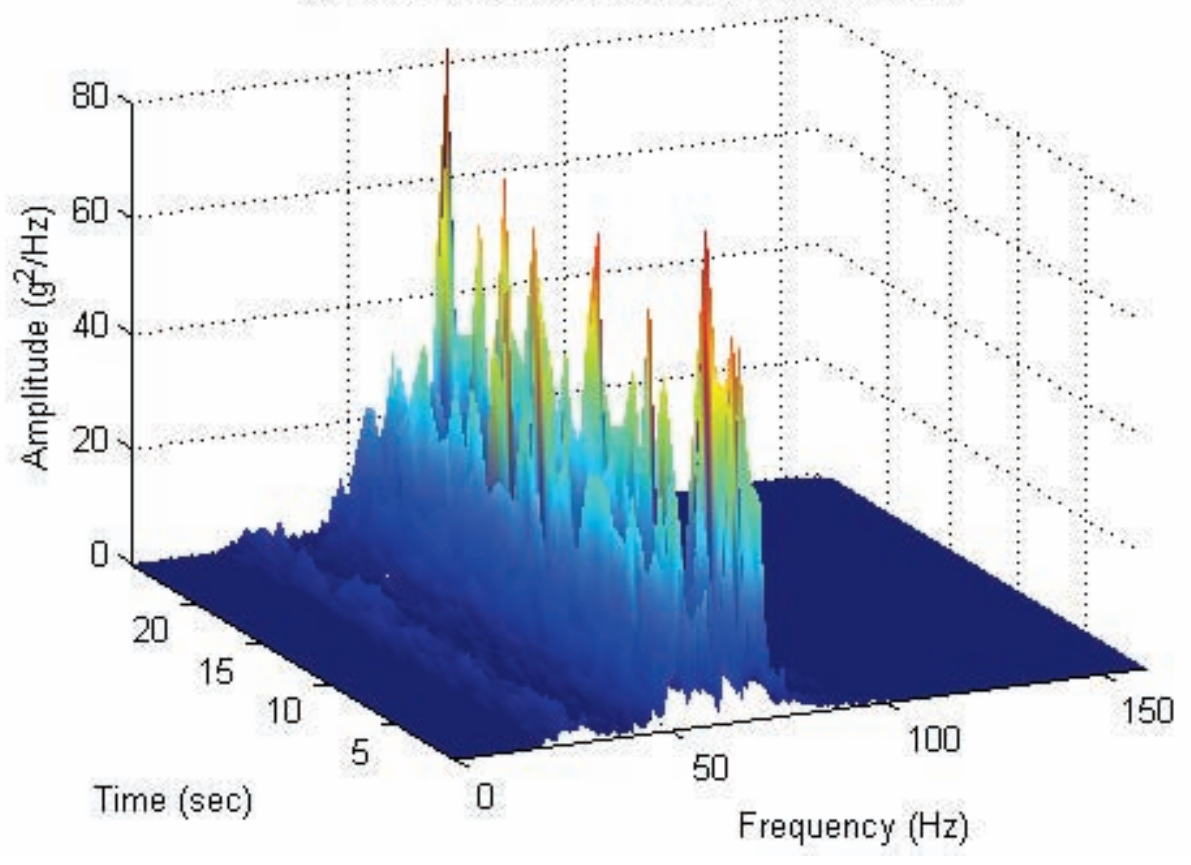

(b) Time-frequency representation

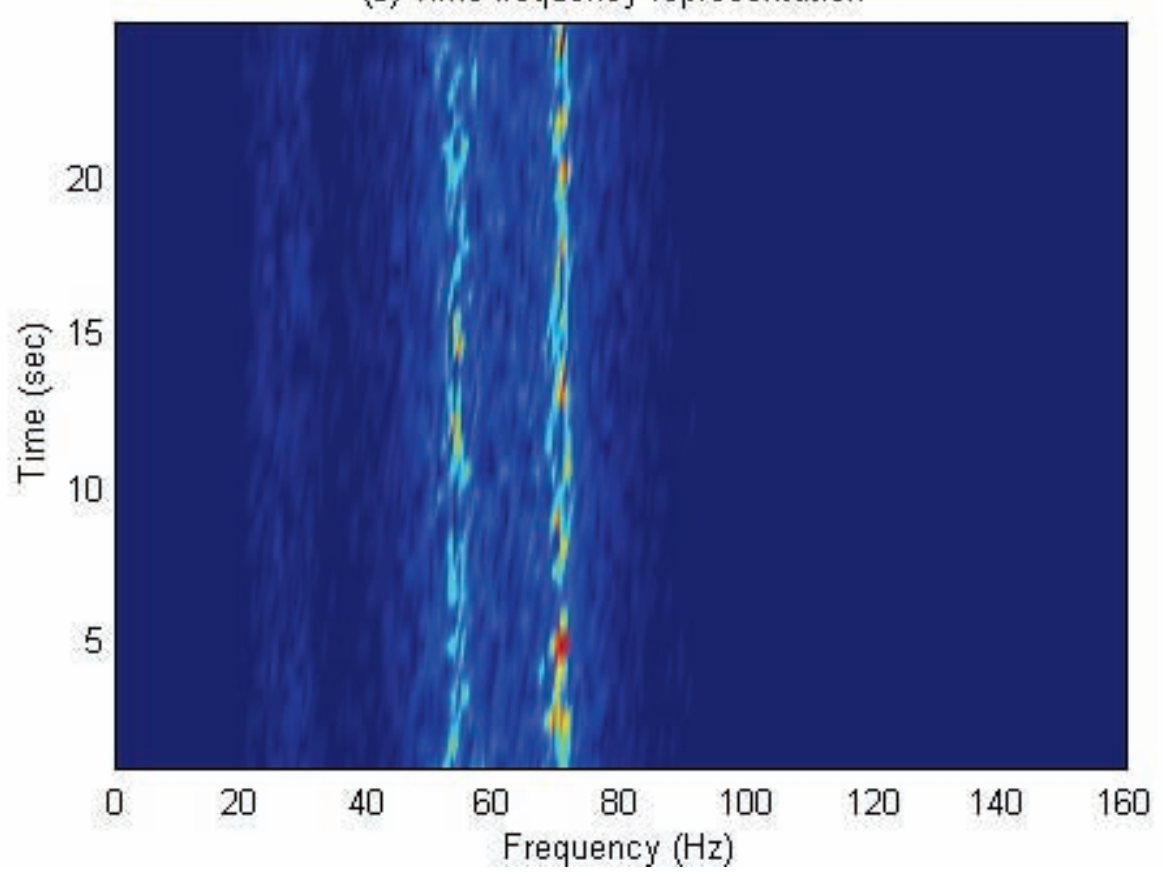

Figure 53: $\quad$ STFT analysis of signal from State \#1, Channel 4. 
(a) Time-frequency-amplitude representation

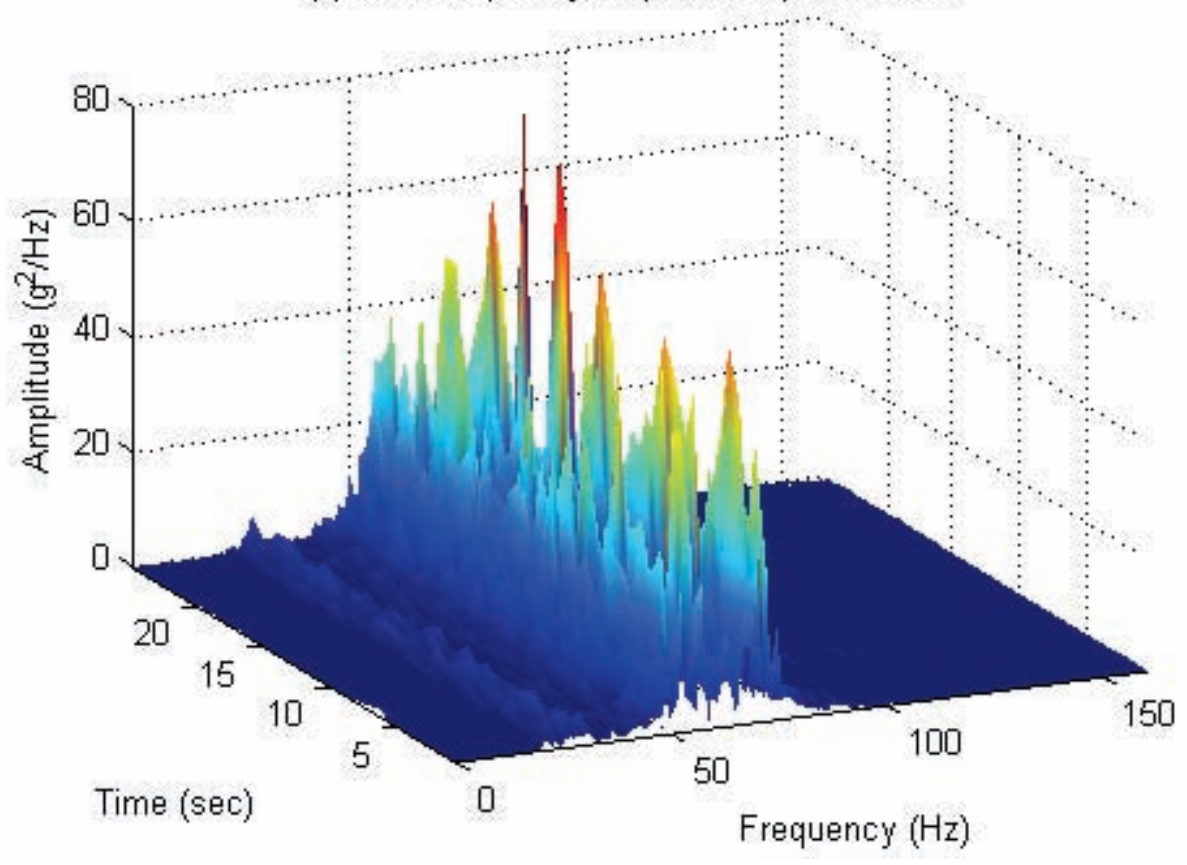

(b) Time-frequency representation

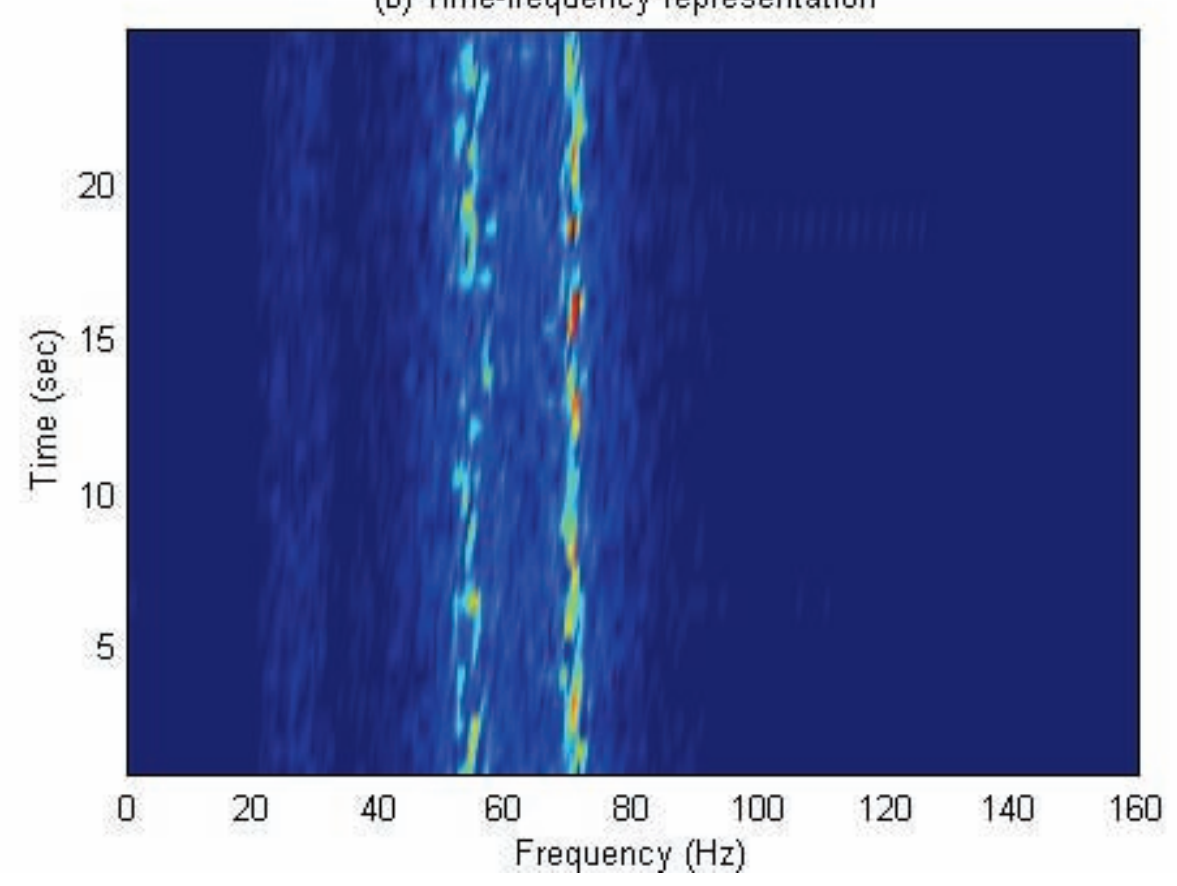

Figure 54: $\quad$ STFT analysis of signal from State \#10, Channel 4. 
(a) Time-frequency-amplitude representation

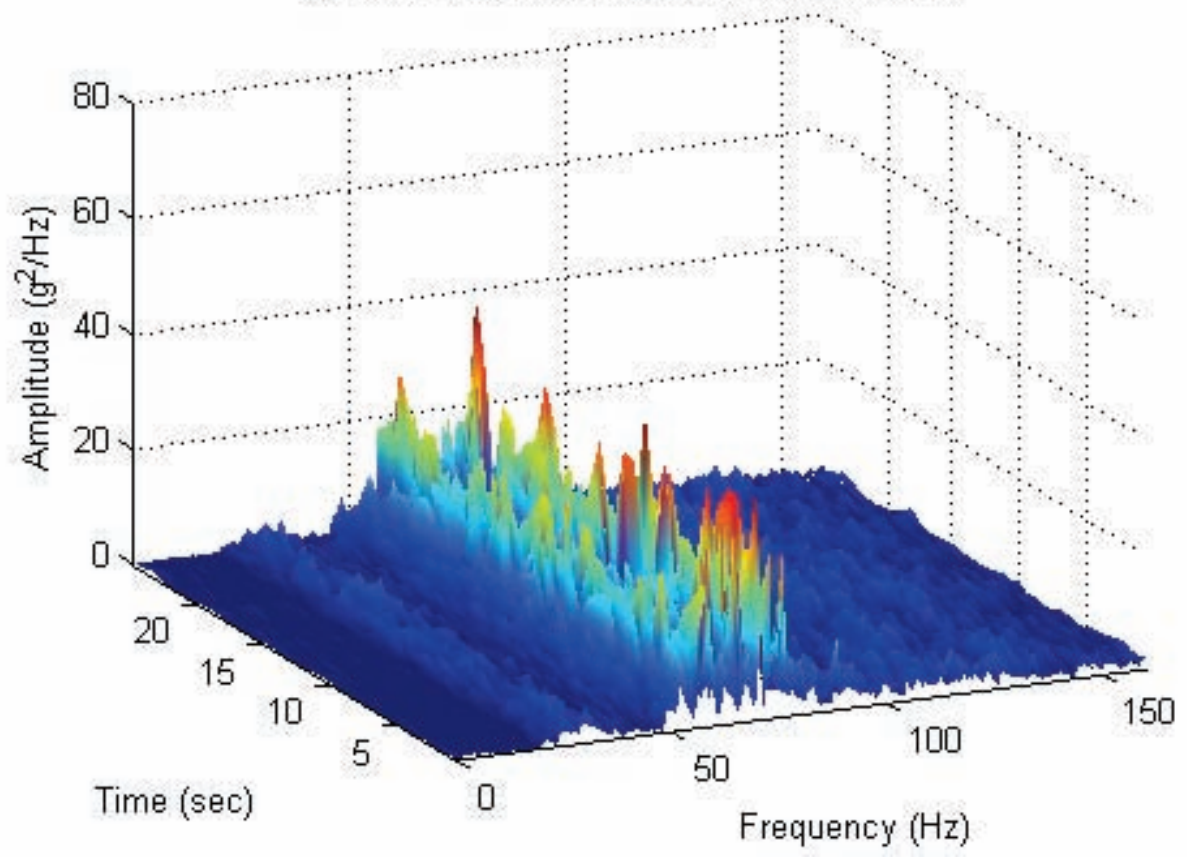

(b) Time-frequency representation

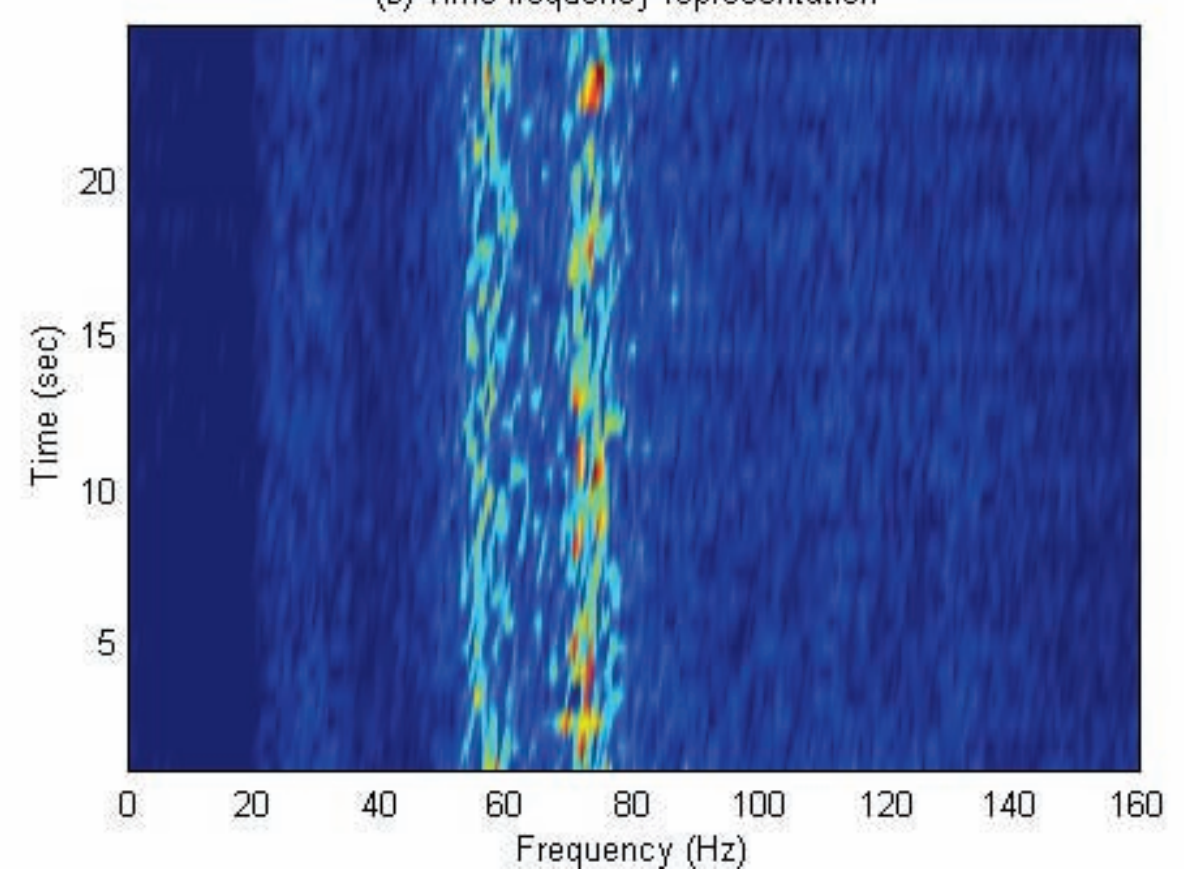

Figure 55: $\quad$ STFT analysis of signal from State \#14, Channel 4. 
(a) Time-frequency-amplitude representation

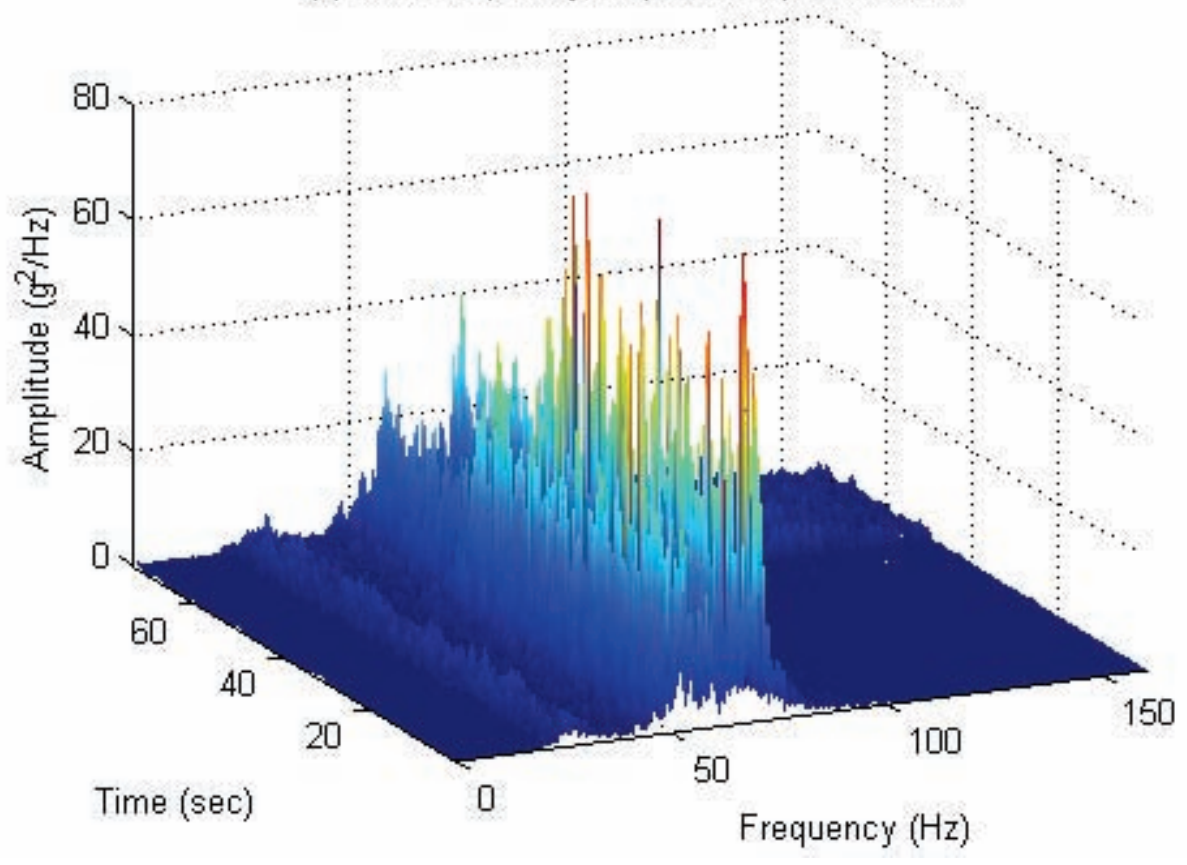

(b) Time-frequency representation

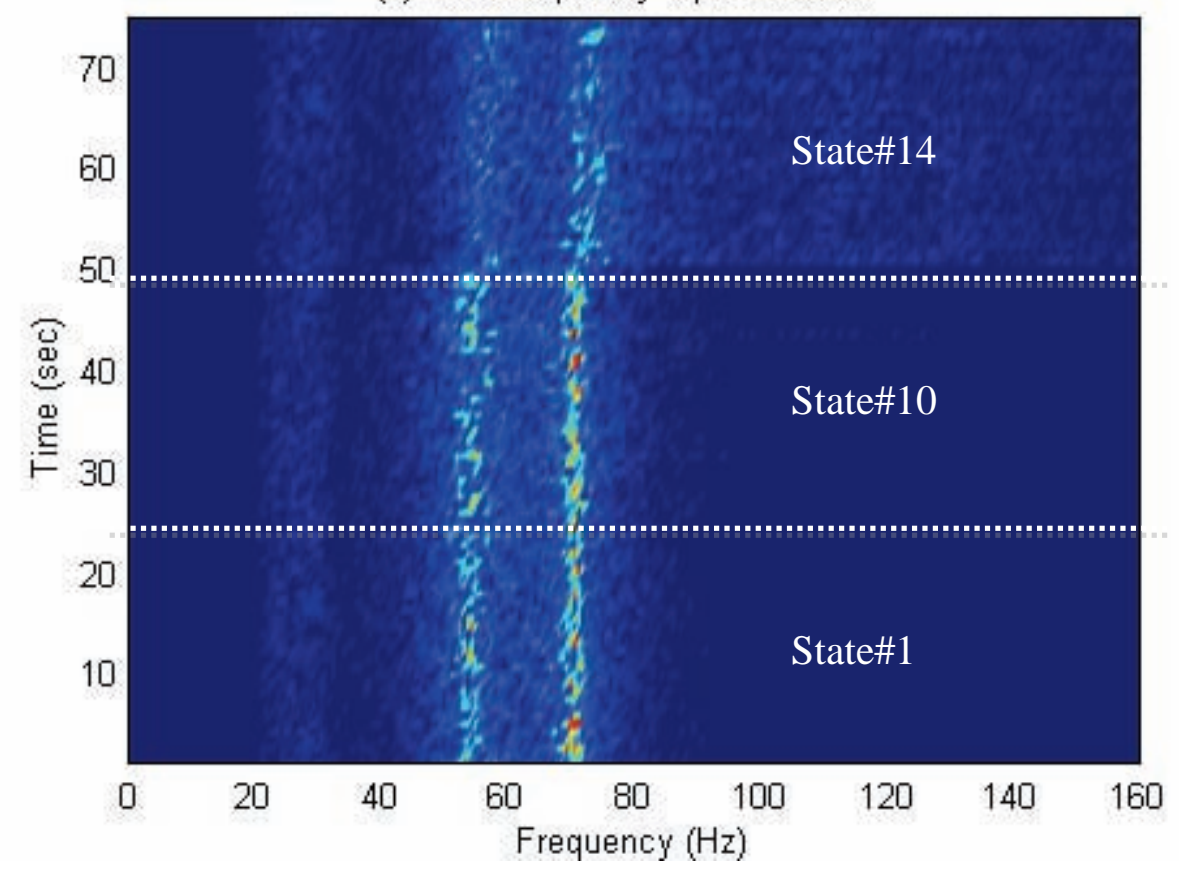

Figure 56: Individual spectrograms in concatenated format, Channel 4.

In conclusion, the STFT analysis does not show any significant changes in the frequency components for the lowest damaged state (State \#10). However, some minor indications of the time-varying nature of the signal's frequency content can be seen for the damaged state corresponding to the most impacts (State \#14), but it would be difficult to base a damage assessment on such subtle changes. Note that, within a given window, the Fourier transform represents the average properties of the signal, and these average properties remain constant throughout the time history. 


\subsubsection{Wavelet Transform}

The WT has been developed to overcome the resolution limitations of the STFT. Recall that in STFT analysis, the time and frequency resolutions are determined by the length of the window, and yield a time-frequency representation of the signal that has constant resolution in time and frequency. However, wavelet analysis uses a different window technique with variablesized length. Actually, the wavelet analysis allows the use of long time intervals for more precise low-frequency information and shorter time intervals to better capture the time-varying nature of the high-frequency information (Misiti et al., 2007). As a consequence, wavelets analysis can have a good time and poor frequency resolution at high frequencies, and good frequency and poor time resolution at low frequencies.

The major advantage of the wavelets is the ability to perform local analysis. In the SHM field, this ability makes the wavelets useful in detecting nonlinearities related with discontinuities in the signal caused by transient processes such as the impacts in this experiment (Robertson et al., 2003).

The continuous wavelet transform (CWT) is defined by convolving the signal $f(t)$ with scaled and shifted versions of the wavelet function $\psi$, as follows:

$$
C(\text { scale, position })=\int_{-\infty}^{+\infty} f(t) \times \psi(\text { scale, position }, t) \times d t
$$

This process produces WT coefficients, $C$, that are a function of two parameters: scale and position. The scale parameter is correlated with the frequency, and it dilates or compresses the wavelet function. In terms of frequency, low frequencies (high scales) correspond to global information of a signal. High frequencies (low scales) correspond to a detailed view of a signal that usually lasts a relatively short time (Polikar, 2007). The parameter position intends to move the wavelet function along the time signal as a moving window. The resulting WT coefficients estimated at different scales and positions provide both frequency and time information about the signal being analyzed.

A wavelet function is a waveform with finite duration and an average value of zero. There are many wavelet functions available in the references (Misiti et al., 2007). Note that the type of wavelet to use is dependent on the signal to be analyzed.

Recall that the impacts cause changes in the high-frequency components for short durations. In order to compare with the STFT analysis, the wavelet analysis carried out in this study uses the same discrete-time signal as before, i.e., the same acceleration-time histories from Channel 4 corresponding to States \#1, \#10, and \#14. A complex Morlet wavelet is used to form the CWT, which consists of 143 scales. Figure 57, Figure 58, and Figure 59 plot the WT coefficients in both time-frequency-amplitude and time-frequency representations. The colour at each point is associated with the magnitude of $\mathrm{C}$, which represents the energy distribution of the signal. A few observations can be made based on the visual inspection of the CWT, as follows:

i. $\quad$ The third and fourth frequency components (54.2 and 70.7 Hz for baseline condition) can be clearly seen in these plots;

ii. The amplitude of the WT coefficients associated with the frequency components at 54.2 and $70.7 \mathrm{~Hz}$ decrease for the damaged State \#14, but other frequency components across the spectrum increase as a result of the nonlinearities associated with the impacts; 
iii. The peaks of the frequency components in Figure 59 for the most damaged condition (State \#14) drift in frequency over time as indicated by the distribution in the higher amplitudes of the WT coefficients at frequencies around 54.2 and $70.7 \mathrm{~Hz}$. Although these same bands can be seen at the lowest damage level (State \#10) they are not as broad in frequency, and this feature is attributed to the reduced number of impacts associated with this damage level.

(a) Time-frequency-amplitude representation

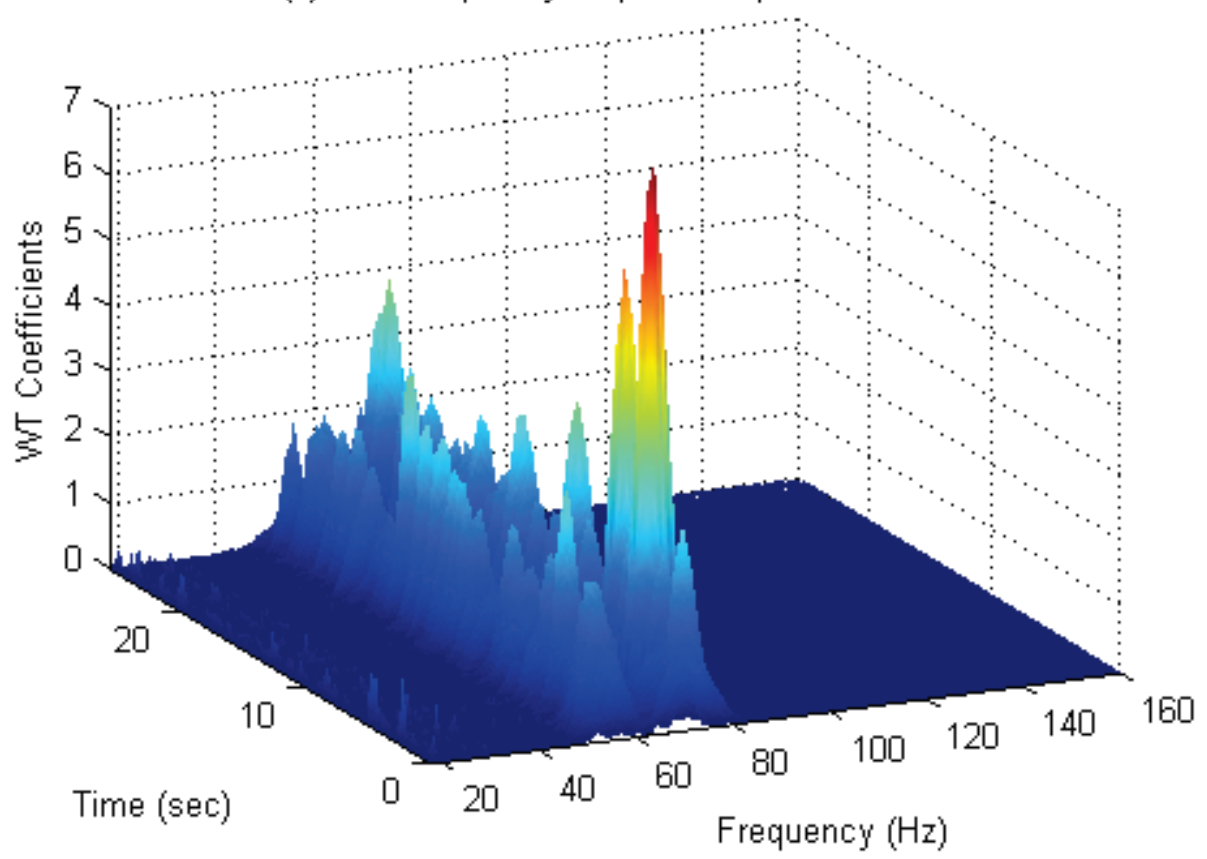

(b) Time-frequency representation

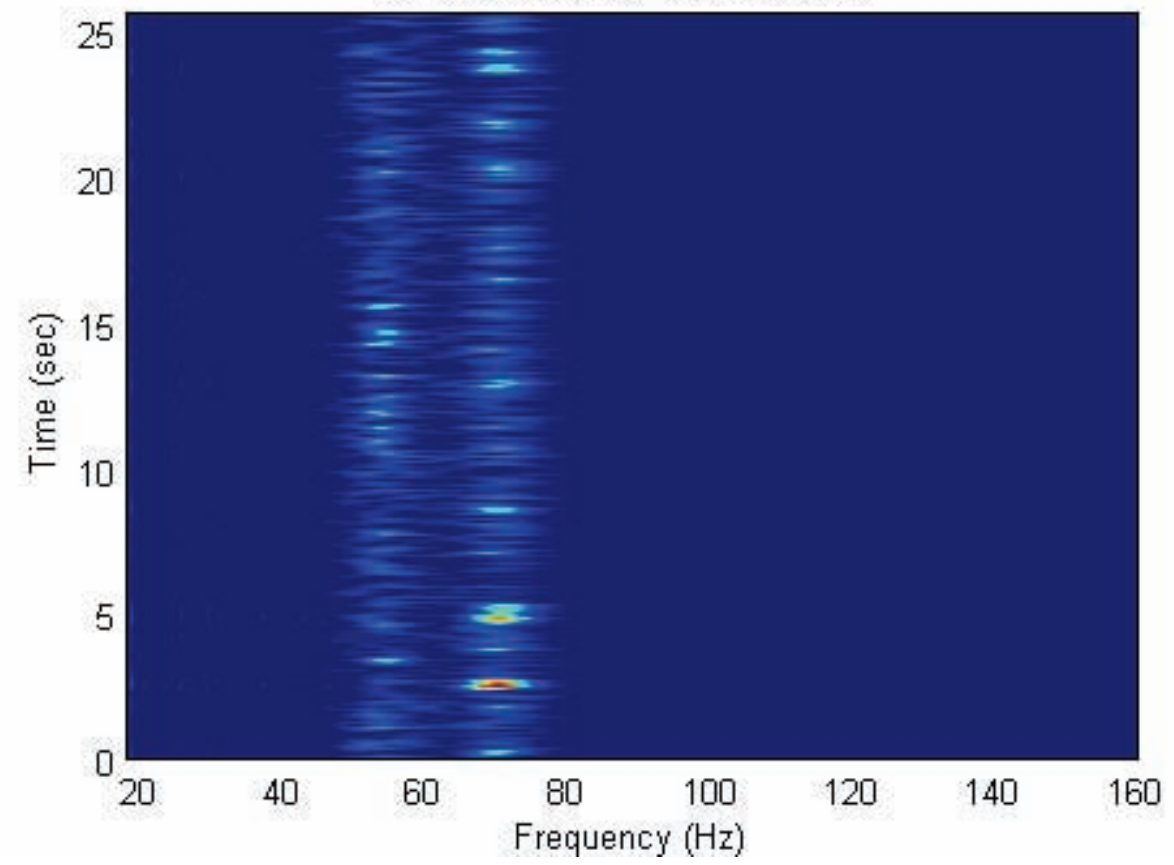

Figure 57: CWT of time history from State \#1, Channel 4. 
(a) Time-frequency-amplitude representation

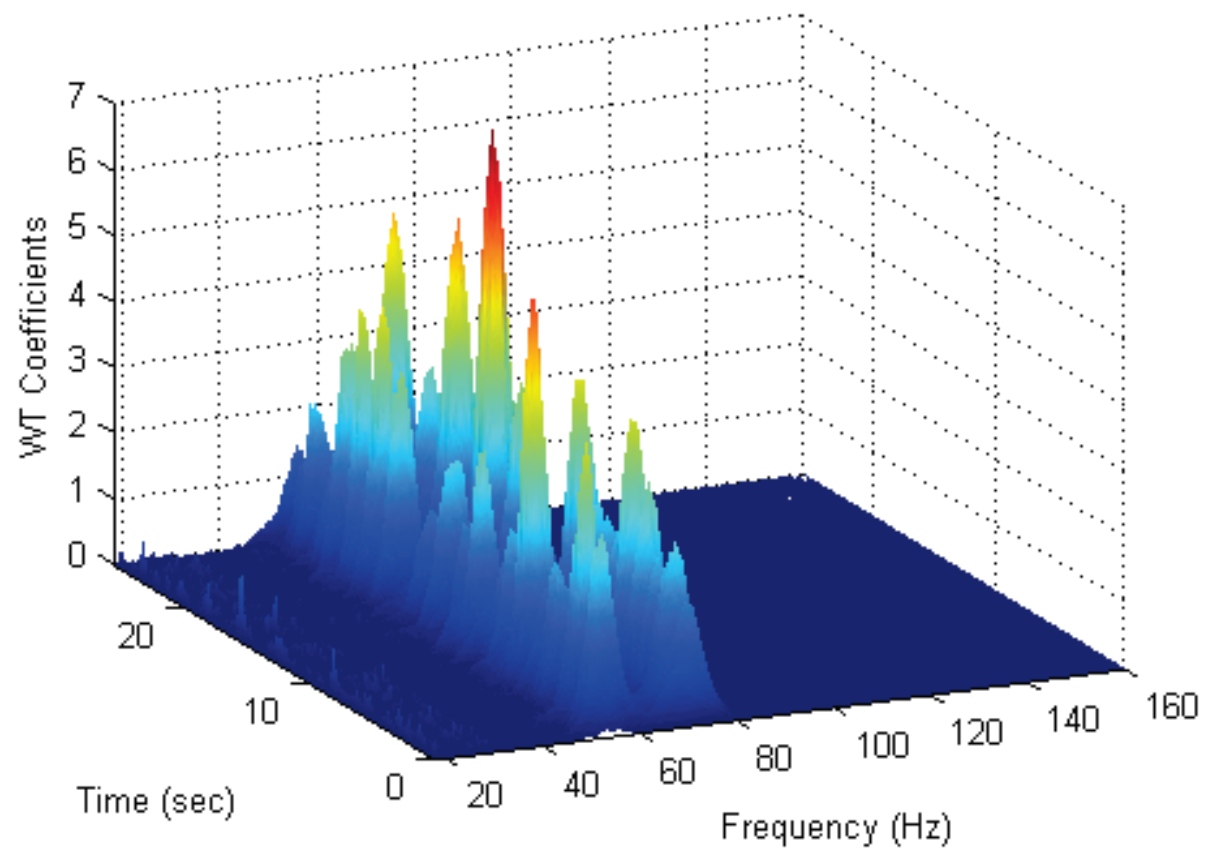

(b) Time-frequency representation

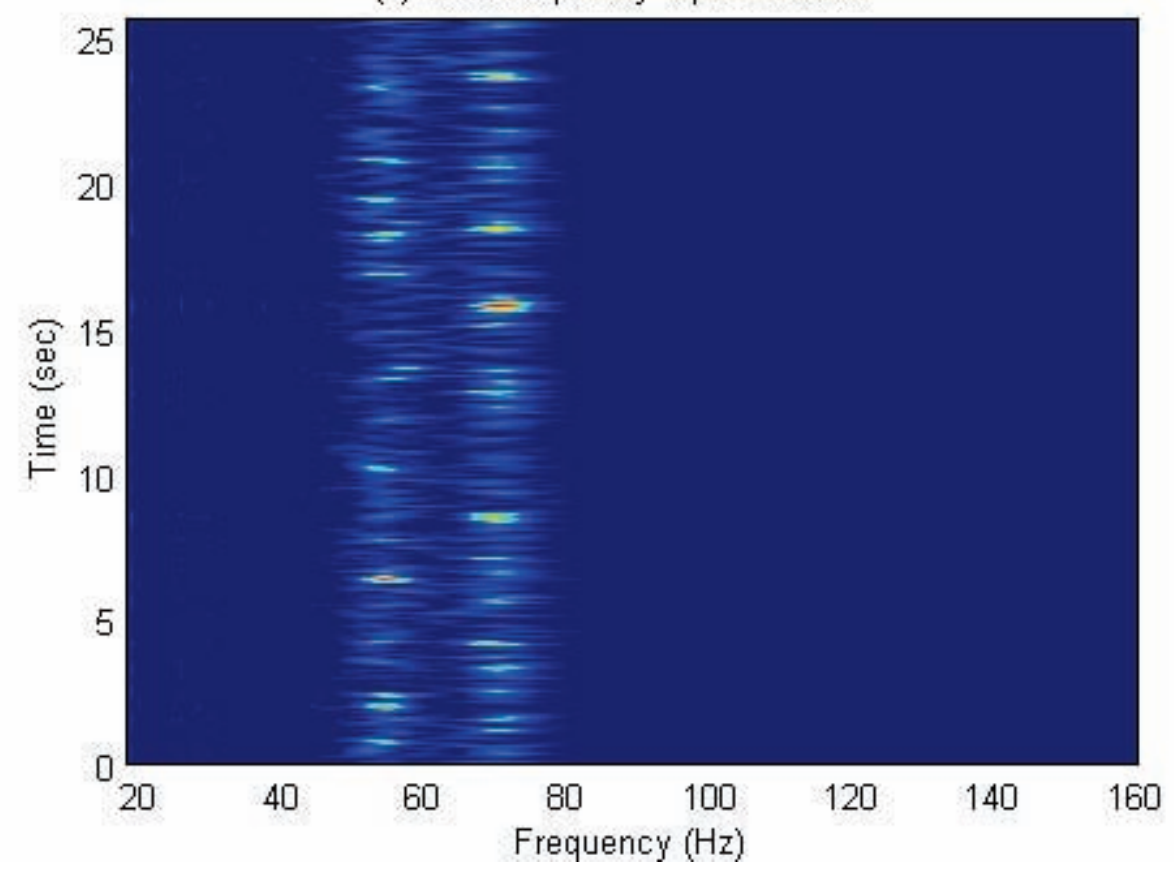

Figure 58: CWT of time history from State \#10, Channel 4. 
(a) Time-frequency-amplitude representation

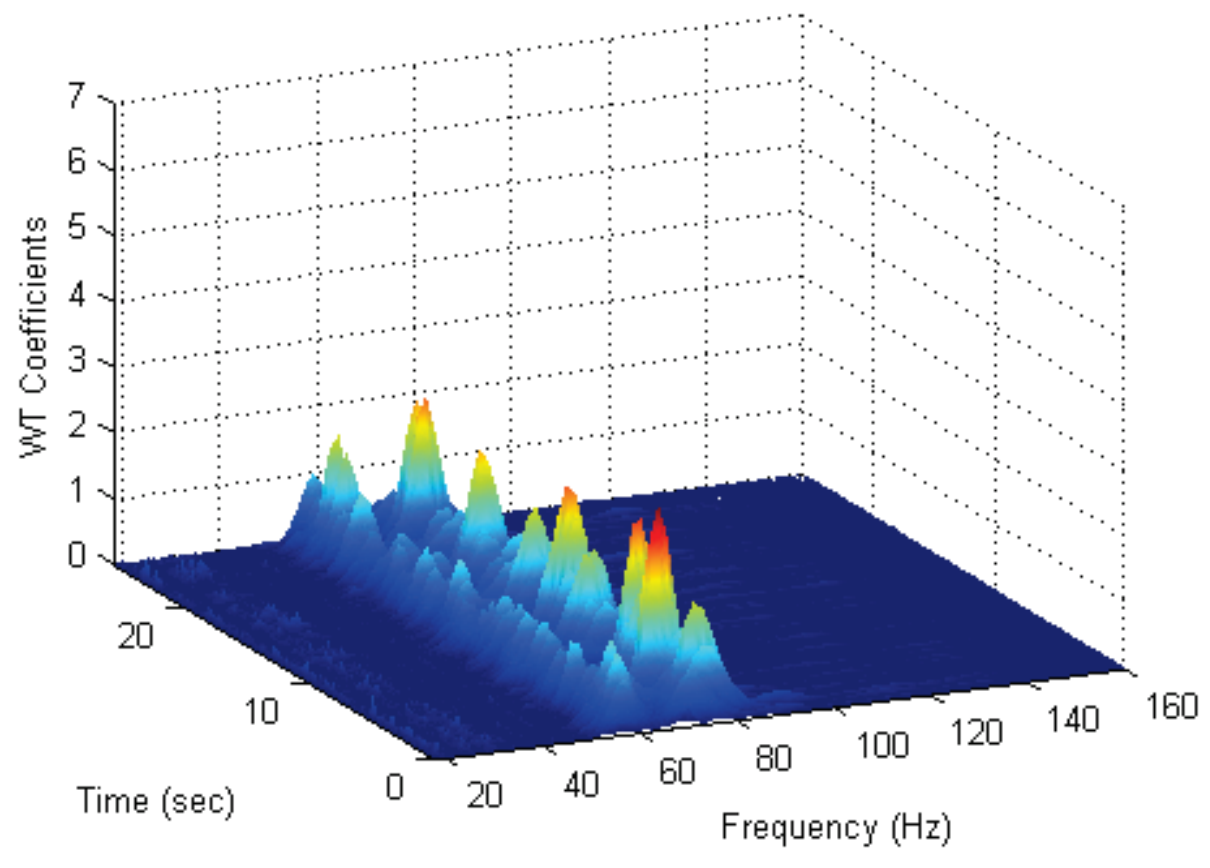

(b) Time-frequency representation

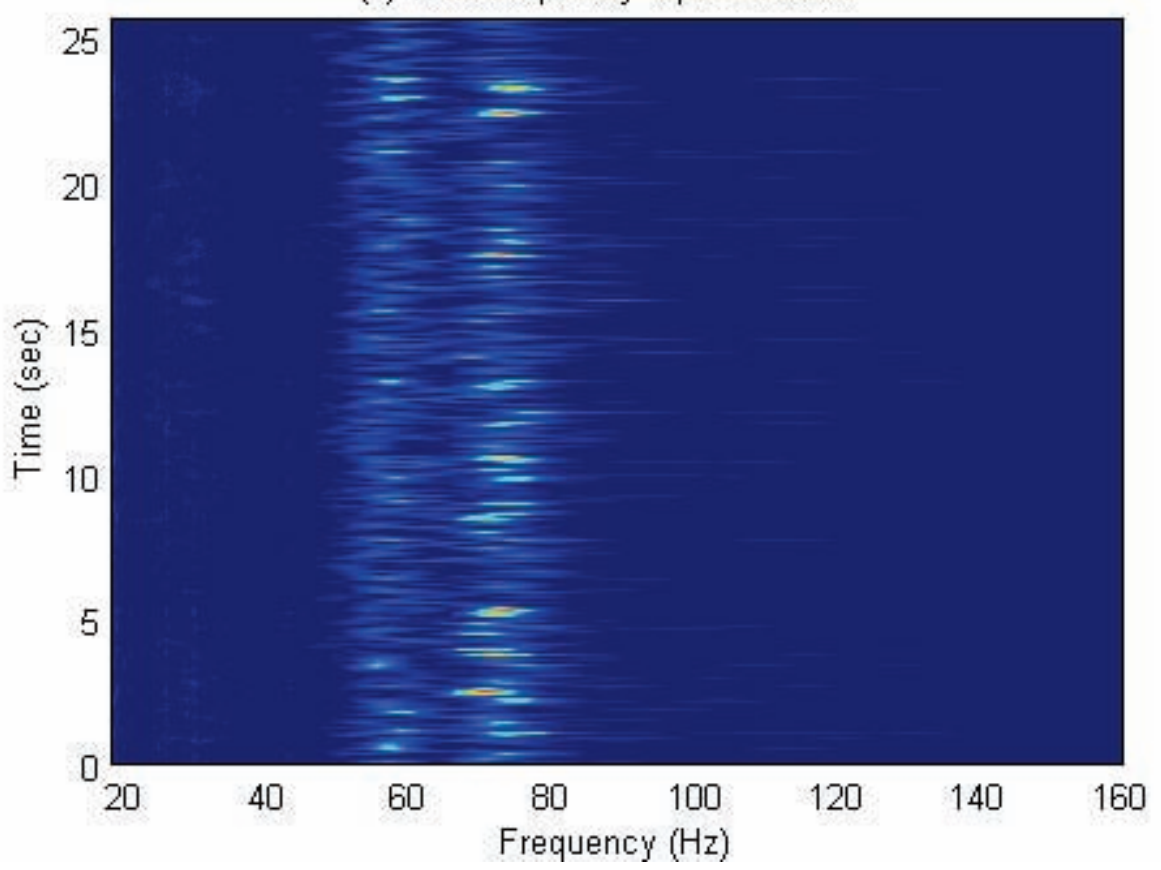

Figure 59: $\quad$ CWT of time history from State \#14, Channel 4. 
Figure 60 (a) and (b) show a limited frequency bandwidth between 45 and $80 \mathrm{~Hz}$ from Figure 57 and Figure 59, respectively. The figures are plotted with a different WT coefficient scale in order to highlight the changes of the frequency components over time. By looking at the time-varying nature of the peaks of the frequency components in both figures, there is an indication that the damaged State \#14 (Figure 60 [b]) corresponds to a nonstationary system because the impacts appear to cause the frequencies associated with peaks in the WT coefficients to vary in time as would be expected in a system where the stiffness alternates between two different states. In contrast, for the baseline condition, these peaks remain relatively constant in frequency throughout the duration of the signal as would be expected for a linear time-invariant system.

The wavelets analysis can also be used to find hidden patterns or singularities in the signal that usually last a short time relative to the duration of the signal. For this purpose, State \#10 is used because of the reduced number of impacts between the bumper and the suspended column. For this state condition, it was observed when measuring the data that the impacts occurred once in a while and with no more than 10 impacts per time history.

As a reference, Figure 61 (a) shows a Channel 4 time history corresponding to State \#10 and Figure 61 (b) shows the AR(30) residual errors squared from a prediction of that time history. The spikes correspond to singularities imposed by the impacts in the time history that the AR(30) model cannot predict. Note that Figure 61 (b) plots the squared errors to amplify the residual errors correlated with those singularities.

Singularities in the time history can be identified by the presence of modulus maxima of the WT coefficients at specific frequency through their evolution along the time axis. For comparison, Figure 62 corresponds to the limited frequency bandwidth between $45 \mathrm{and} 80 \mathrm{~Hz}$ in time-frequency representation of the Figure 58. The impacts can be identified in Figure 62 by comparing the spikes with those in Figure 61 (b). Moreover, the impacts are more correlated in time with the third frequency component ( $54.2 \mathrm{~Hz}$ for the baseline condition), suggesting that the bumper's impacts most influence the third mode. However, it should be pointed out that there is not a direct one-to-one correlation between peaks in the residual error plot and the peaks in the modulus of the WT coefficient plot, indicating a need to validate these features with information about the true number of impacts.

In conclusion, the CWT confirms some of the observations made with the previous STFT analysis about the nonstationary nature of the response corresponding to the most damaged condition (State \#14). However, even though theoretically the CWT has better time resolution at higher frequencies than the STFT, it does not have the resolution in time to establish that the lower level of damage (State \#10) corresponds to a nonstationary system. Although the results do not allow one to conclude that State \#10 is from a nonstationary system, the CWT did reveal changes in the WT coefficient amplitudes that can be correlated with the bumper's impacts when compared to changes in the residual errors of an AR model.

\subsubsection{Holder Exponent}

Mathematically, the Holder exponent is a measure of the signal's regularity. Because singularity points have no continuous derivatives, the singularities can be identified when the Holder exponent suddenly drops to a value of zero or below.

In SHM, the Holder exponent can be used to identify damage that introduces discontinuities into the measured dynamic response data (Robertson et al., 2003). Basically, this technique indicates the presence of singularities and identifies when they occur. As a 
consequence, the drops in the Holder exponent can be used as damage-sensitive features. In realworld structures, it can potentially be used to identify singularities associated with cracks that open and close during dynamic loading.
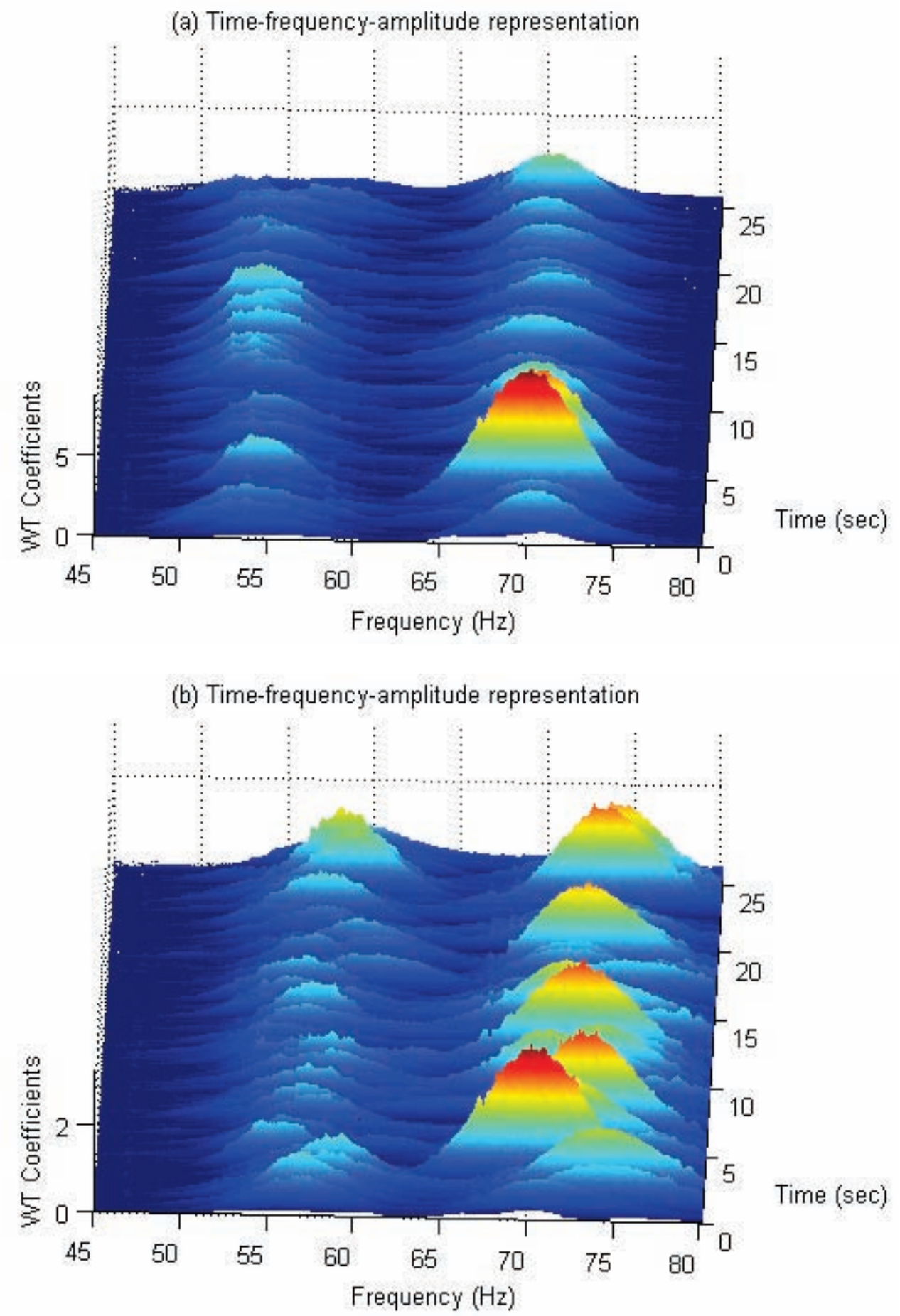

Figure 60: Details of the CWT for the time histories from State \#1(a) and State \#14 (b), Channel 4. 
(a) Time history of Channel 4 from State\#10

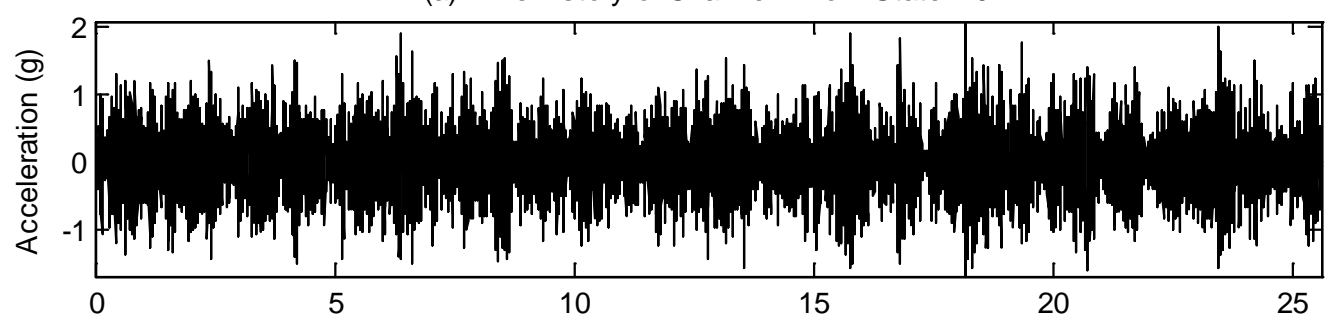

(b) $A R(30)$ residual errors squared

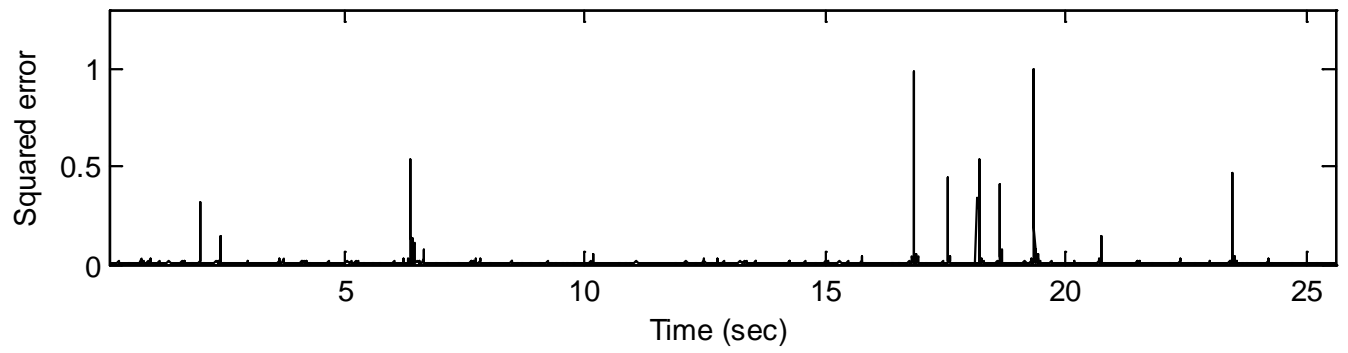

Figure 61: AR(30) model residual errors of one time history from Channel 4 of State \#10.

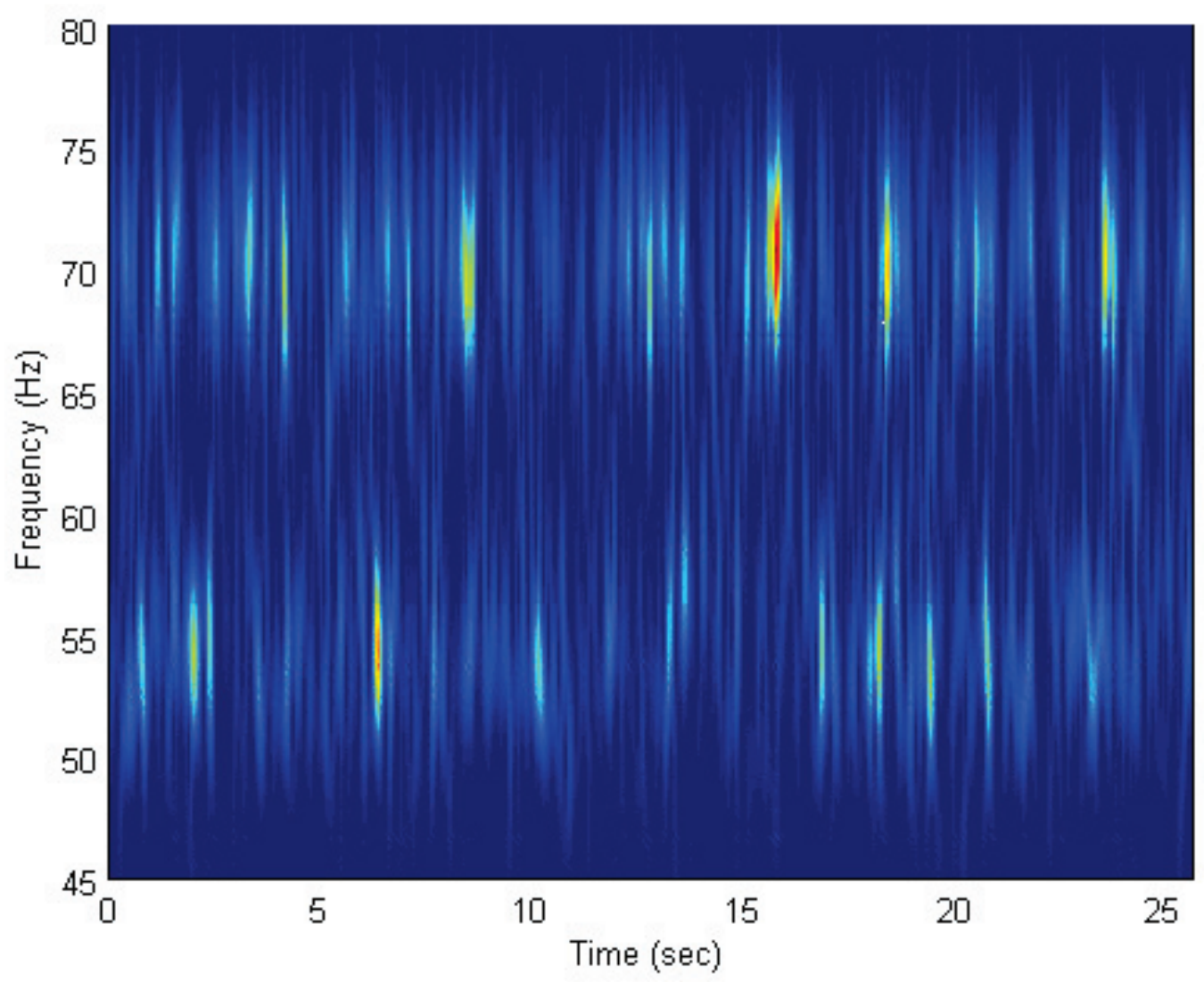

Figure 62: Details of the CWT for the time history from State \#10, Channel 4. 
In this study, the Holder exponent is used to identify singularities in the time histories imposed by the bumper. A procedure is presented for capturing the time-varying nature of the Holder exponent based on WT by applying it to potential nonstationary random time histories from the damaged state conditions.

Figure 63 (a) is a portion of the time history from Channel 4 of State \#10 plotted in Figure 61 (a). Note that indications of impacts cannot be seen in that figure. The plot of the Holder exponent function calculated from the State \#10 time history is shown in Figure 63 (b). Recall from Figure 61 (b) that the AR(30) residual errors can be used to identify discontinuities. Figure 63 (c) and (d) plots the same portion of the AR(5) and AR(30) residual error data, respectively. Red circles mark the significant drops in Holder exponent values and the corresponding spikes in the AR residual errors associated with potential impacts. Although the drops in the Holder exponent function are fairly apparent to the naked eye, identification of them using threshold lines is difficult because of the considerable variability associated with the Holder exponent. 
(a) Portion of time history from State\#10

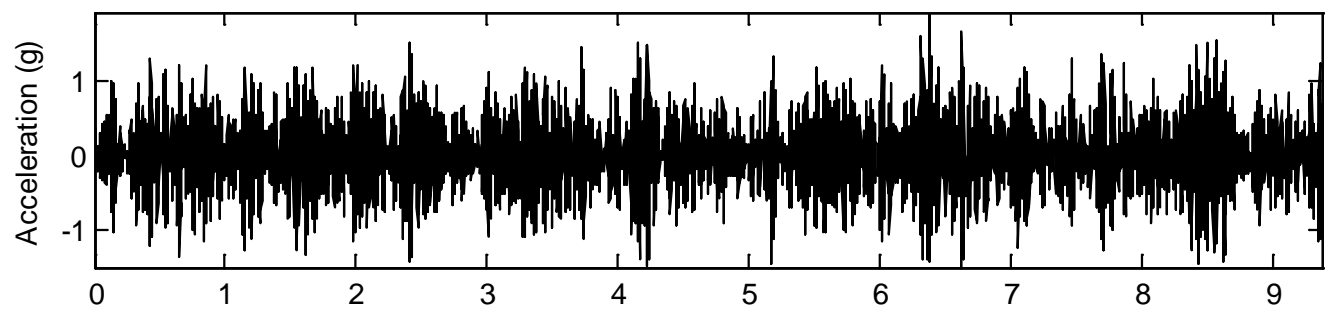

(b) Holder exponent function

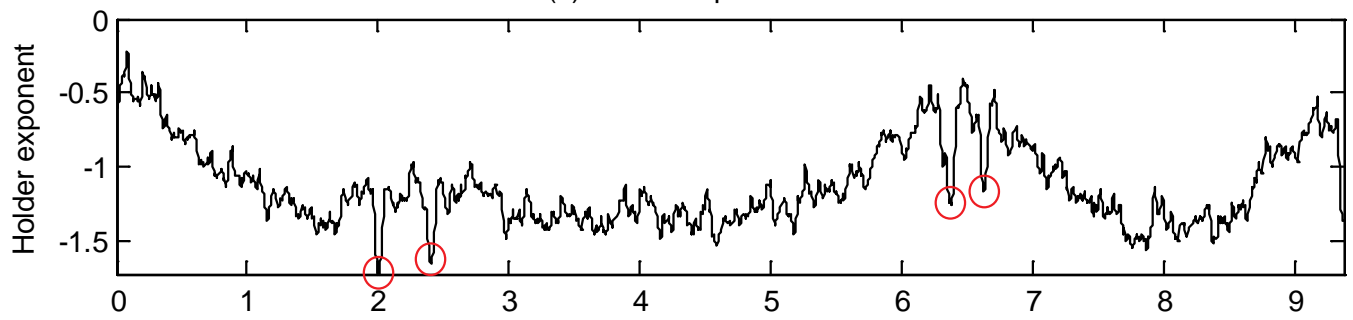

(c) $A R(5)$ residual errors squared

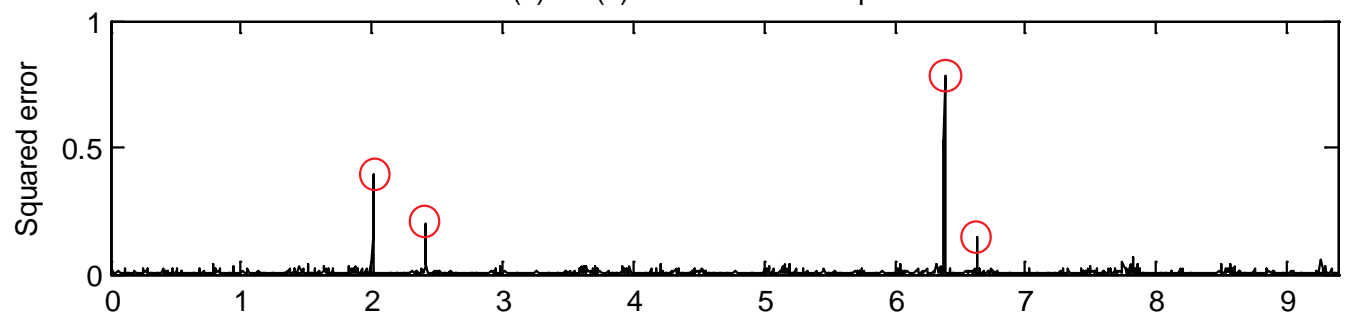

(d) $A R(30)$ residual errors squared

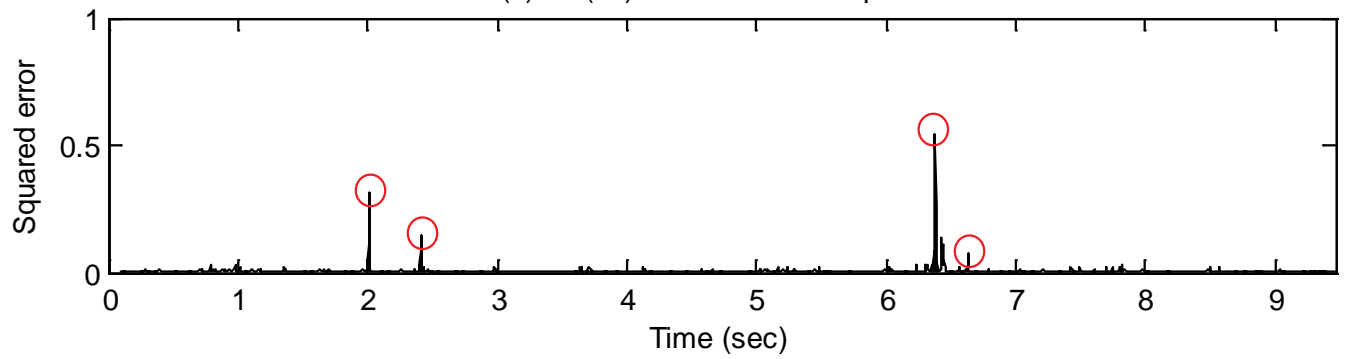

Figure 63: Portion of a time history from State \#10 (Channel 4) with the associated Holder exponent function and residual errors from both AR models.

In conclusion, the Holder exponent technique can be used to extract damage-sensitive features when damage introduces discontinuities into the measured dynamic response data. However, it is necessary to develop statistical classifiers in order to detect the presence of such singularities. For State \#10, considering the previous conclusions from the time-frequency analysis, the Holder exponent and AR residual error results show that the impact damage is better detected with a time-domain approach as opposed to a frequency-domain approach. The frequency and time-frequency domain approaches do not have sufficient time resolution to capture an individual impact and, as such, these effects tend to be treated only in an average sense with these methods. 


\section{CLASSIFICATION ALGORITHMS}

The last section presented statistical techniques that are concerned with extraction of damage-sensitive features from dynamic response data. A damage-sensitive feature was defined as some quantity extracted from the measured system response data that is correlated with the presence of damage in a structure. This section is concerned in developing statistical models to classify those damage-sensitive features in order to enhance the damage-detection process.

The algorithms used in statistical model development usually fall into three general categories: (1) Group classification; (2) Regression analysis; and (3) Outlier detection. The appropriate algorithm to use depends on the ability to perform supervised or unsupervised learning. Here, supervised learning refers to the case where examples of data from damaged and undamaged structures are available. In this case, the classes are predefined and a set of labelled data is used to develop the algorithms that classify future data as belonging to either the undamaged or damaged class in a discrete manner. With appropriate data, this approach can be used to generate a regression model that performs a more continuous classification where new features may be assigned to a particular damage level. Note that such an approach is predicated on having data and the associated features available from the different damage levels. Unsupervised learning refers to the case where data are only available from the undamaged structure. In this case, only one data class is known, and algorithms are developed to identify subsequent features that are outliers to this class.

In this section, three techniques are applied in an unsupervised learning mode to classify the data obtained from the test structure, namely SPC, cluster analysis, and factor analysis (FA). These techniques differ in the way they perform the learning task. The SPC models the underlying distribution of the baseline condition to define the threshold limits. Then, the discrimination of undamaged and damaged state condition is based on the number of features falling beyond the threshold limits. On the other hand, in cluster analysis and FA techniques, the classes are discovered from the underlying similarity among the data.

\subsection{Statistical Process Control}

The SPC techniques may be applied for feature classification in an effort to discriminate the damaged from the undamaged state conditions. SPC uses control charts for monitoring whether the process is operating in statistical control. The process is said to be in control when the process data vary randomly within the control limits (or threshold values). The purpose is to detect any abnormal changes in the process. These changes are observed as abnormal points on the charts that caused changes in the mean and/or variance of the data. Abnormal conditions are identified by a statistically significant number of points exceeding the control limits as well as by systematic changes to the data within the control limits (e.g., the data are no longer randomly distributed within the control limits). Control charts often make assumptions of normality (the data have a normal distribution) and independence (data are not correlated) for better performance (Grant et al., 1988; Montgomery, 1997; Gupta et al., 2007).

In this study, the process is defined by the dynamic response characteristics of the test structure, and it is expected that damage, particularly that which introduces nonlinear response characteristics, will produce changes in the system's responses that can be identified as abnormal points in the control charts. 


\subsubsection{Statistical Basis of the Shewhart X-bar Control Charts}

The Shewhart X-bar control charts are used to identify when data points fall outside the control limits (throughout this report, these points are also called “outliers”). A statistically significant amount of points outside the control limits is an indication that a process is out of control. Physically, it is an indicator that the structure has an unusual source of variability that deviates it from the baseline condition. On the other hand, if data points fall inside the control limits, the process is said to be in control. Note that there are cases where all data points fall inside the control limits, and the process is not in control, as will be explained later. A brief theoretical description of X-bar control charts is described below.

Suppose a random variable $X$ that is characterized by a normal probability distribution described by its parameters: mean $\mu$ and standard deviation $\sigma$. If $x_{1}, \ldots, x_{n}$ is a sample of size $n$ and $\bar{X}$ is the average of this sample, then based on the central limit theorem, the samples means $\bar{X}_{i}$ are normally distributed with mean $\mu$ and the standard deviation $\sigma_{\bar{x}}=\sigma / \sqrt{n}$. Thus, in $100(1-\alpha) \%$ of the cases, the sample mean $\bar{X}_{i}$ will fall between the following interval:

$$
\mu-Z_{\alpha / 2} \frac{\sigma}{\sqrt{n}} \leq \bar{x}_{i} \leq \mu+Z_{\alpha / 2} \frac{\sigma}{\sqrt{n}},
$$

where $Z_{\alpha / 2}$ is a parameter related to the confidence interval, and $\alpha$ is the desired significant level. The standard deviation of the distribution of the $\bar{x}_{i}$ values is $\sigma / \sqrt{n}$, i.e., the standard deviation of the population divided by the square root of the sample size. It is also commonly referred to as the standard error of the mean. The limits are a function of data acquired when the process is thought to be in control and may not necessarily have any direct relationship to the actual process performance. Generally, the parameters $\mu$ and $\sigma$ corresponding to the underlying process are unknown, and it is necessary to estimate these parameters from samples when the process is thought to be in control.

One method to estimate the parameters of the probability distribution is as follows. Suppose that $m$ samples of $X$ with $n$ observations each are available. The best estimator of $\mu$ is given by

$$
\hat{\mu}=\frac{1}{m} \sum_{i=1}^{m} \bar{x}_{i},
$$

where $\bar{x}_{i}$ is the sample average value for the $i^{\text {th }}$ sample. The standard deviation can be estimated from either the $m$ standard deviations or the ranges of the $m$ samples (Montgomery, 1997). By using the former one, an estimate of standard deviation, $\hat{\sigma}$, can be obtained by averaging the $m$ standard deviations $S_{i}$.

$$
\hat{\sigma}=\frac{1}{m} \sum_{i=1}^{m} s_{i} .
$$

With these estimates of the process mean and standard deviation, the control charts limits (UCL-upper control limit, CL-centerline, and LCL-lower control limit) are defined as follows for the following $3 \sigma$ confidence intervals: 


$$
\begin{aligned}
& U C L=C L+3 \frac{\hat{\sigma}}{\sqrt{n}} \\
& C L=\hat{\mu} \\
& L C L=C L-3 \frac{\hat{\sigma}}{\sqrt{n}}
\end{aligned} .
$$

Setting the control limits requires one to make a tradeoff between false-positive (indications of damage when none is present) and false-negative (no indication of damage when damage is present) indications of damage. In this study $99.73 \%$ confidence intervals of a normal distribution corresponding to three standard deviations from the mean were chosen for the control limits. Thus, in Eq.(35), $Z_{\alpha / 2}$ is replaced by 3 (Montgomery, 1997). The quantity plotted is the sample average $\bar{X}_{i}$, therefore, the chart is usually called an X-bar chart.

\subsubsection{Control Charts with Autocorrelated Process Data}

As stated above, control charts are developed based on the assumption that the data generated by the process when it is thought in control are normally and independently distributed. Moreover, an out-of-control process manifests itself with changes in the mean and standard deviation. However, control charts can lead to many false alarms when the observations are not independent and positively correlated (Montgomery, 1997).

Unfortunately, the assumption of independent and uncorrelated observations is not valid for the measured acceleration data, as shown in Figure 37. The autocorrelation shown for the baseline condition (State \#1) is certainly large enough to affect the efficacy of control charts. One approach for dealing with correlated data is to first model the data using time series models, for instance, the AR models, and then to apply the control charts to the AR residual errors between the estimated and measured data. This approach assumes that the residual errors given by Eq. (18) are normally and independently distributed with mean zero and standard deviation $s$. For instance, Figure 39 (a) shows that after fitting an AR(30) model to a time history from Channel 5 of State \#1, and using that model to predict the same time history, the associated AR residual errors have practically no correlation. However, two final remarks are made regarding the correlation among the residual errors. First, even though there is no correlation among the residual errors associated with the AR(30) model, the same is not valid for the AR(5) model, as highlighted in Figure 38 (a). This is one indication that the AR(30) should have fewer outliers for the normal condition states. Second, the correlation highlighted in the autocorrelation functions (Figure 39) and lag plots (Figure 42), shows a high degree of correlation in the residual errors from the damaged states. This correlation implies that the impacts introduce some kind of pattern in the response that can make the process appear to be out of control.

For the reasons highlighted above, the control chart analysis is performed using the AR residual errors. For comparison purposes, residual errors from both AR(5) and AR(30) models are examined.

\subsubsection{Control Chart Analysis}

The Shewhart X-bar control charts are constructed based on the subgroups of residuals from the AR models. The implemented process can be summarized as follows. First the AR parameters are estimated by fitting an AR model to an acceleration-time history from Channel 5 of the baseline condition (State \#1). Then, for each state condition, the AR residual errors between the predicted and the measured time histories are calculated and reorganized in 
subgroups of 4 . Subgroups of 4 or 5 data points each are recommended, as is discussed in Montgomery (1997). Before dividing the residuals into subgroups, they are normalized by subtracting the mean and dividing by the standard deviation of the residuals from the baseline condition. This data normalization procedure based on the baseline condition is applied to all residual error data sets that are analyzed. Second, the upper and lower control chart limits are calculated as described in Section 5.1.1, based on the sample mean and standard deviation of the baseline condition. Third, an X-bar control chart is constructed for each state with the subgroups of residual errors as inputs. Note that each subgroup is represented by one data point and the centerline of the charts is zero because the sample mean of the associated normalized residual errors is zero, as described above. The upper and lower control chart limits correspond to 99.73\% confidence intervals, implying that approximately $0.27 \%$ of the data points from normal condition states can be expected to fall outside of the control limits. For 2046 data points, these limits imply that about 6 points should fall outside of those limits.

In order to check the influence of the AR model order on the stability of the process, the control charts are plotted for all the state conditions using both AR(5) and AR(30) models. Figure 64, Figure 65, and Figure 66 show the X-bar control charts using the grouped AR(5) residual errors. On the other hand, Figure 67, Figure 68, and Figure 69 plot the X-bar control charts using the grouped AR(30) residual errors. Figure 70 summarizes the number of outliers falling beyond the control limits for both models. The threshold horizontal dashed line is set up based on the maximum number of outliers present in all the undamaged states (State \#1-\#9). In this case, the threshold is conditioned by State \#9.

As shown in Figure 70 for residual error data obtained with either the AR(5) or AR(30) models, the number of outliers beyond the control limits seems to increase for the damaged state conditions. These results indicate that some unusual source of variability is present in the damaged states. The threshold horizontal dashed line can be used to examine the relative performance of the AR(5) and AR(30) models. In the case of the AR(30) model, the number of outliers associated with all damaged states are greater than the ones associated with undamaged states (State \#16 is on the transition border). In the case of the AR(5) model, some damaged states have a number of outliers that are within those found for the undamaged states, such as States \#10, \#11, and \#16. The better results for the AR(30) model might be associated to the correlation among the residual errors within the damaged states as highlighted in Figure 39. 

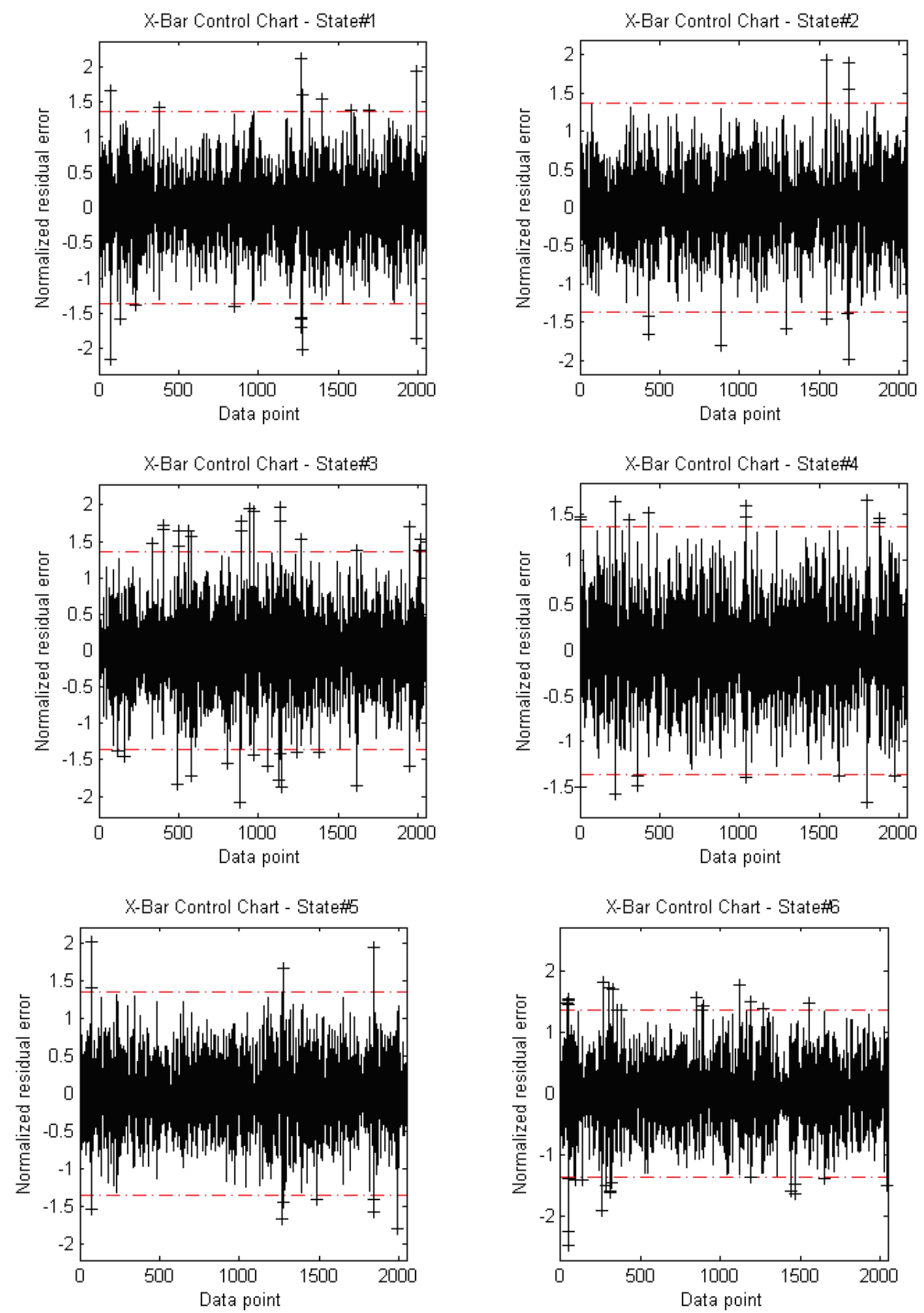

Figure 64: The X-Bar control plot of the mean of the grouped AR(5) residual errors (Channel 5). 
X-Bar Control Chart - State\#

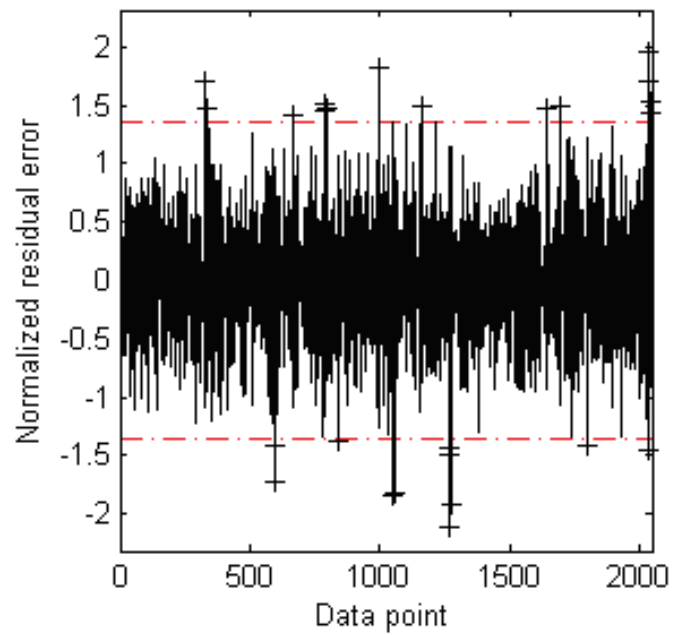

X-Bar Control Chart - State\#g

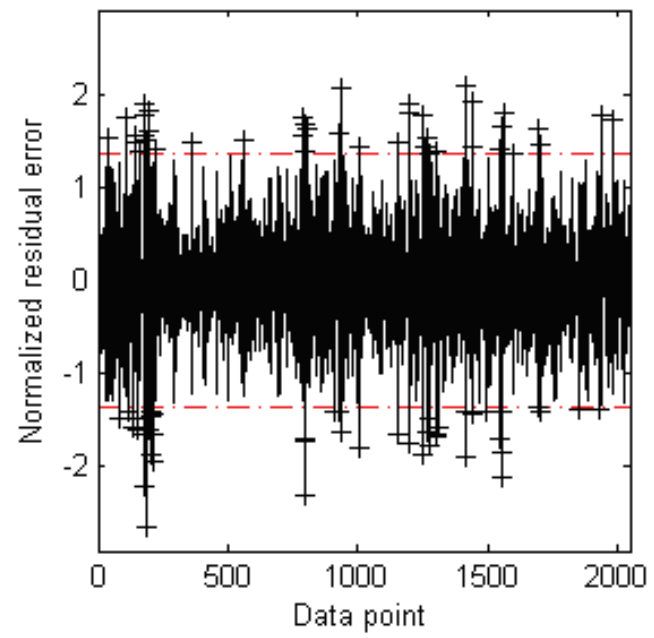

X-Bar Control Chart - State\#11

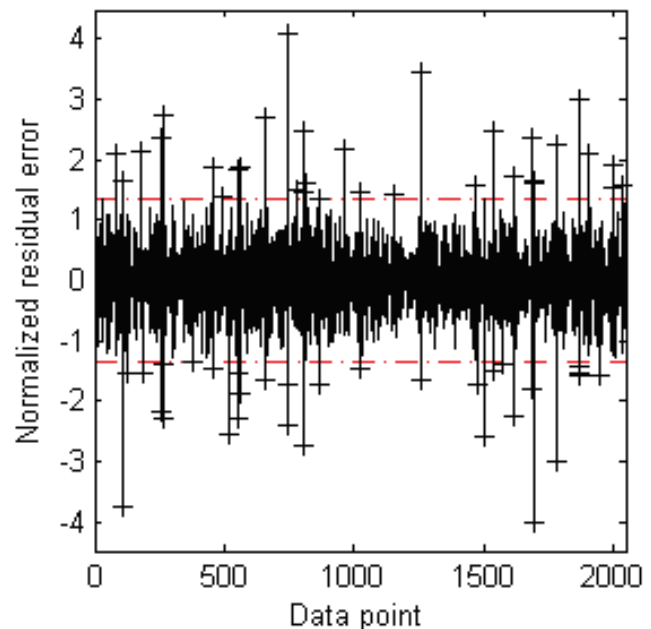

X-Bar Control Chart - State喏

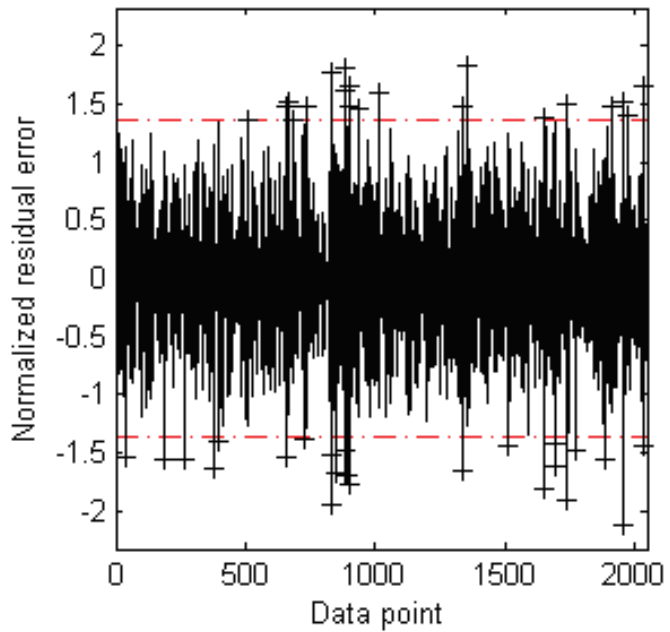

X-Bar Control Chart - State\#10

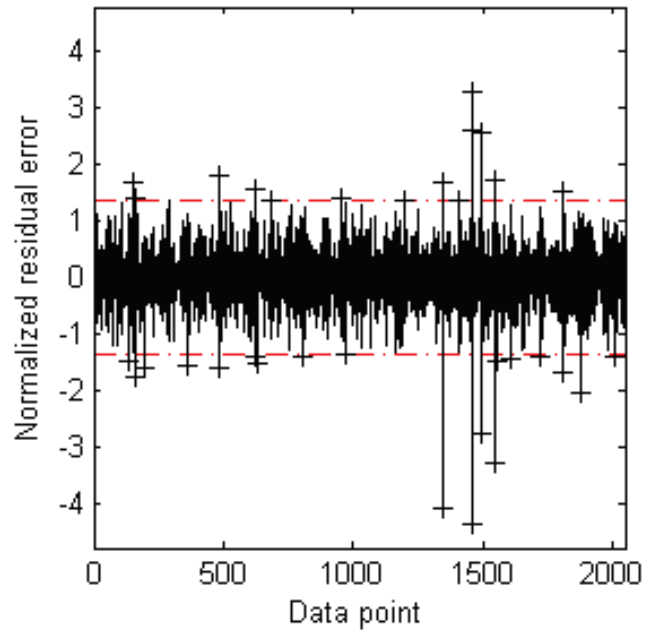

X-Bar Control Chart - State\#12

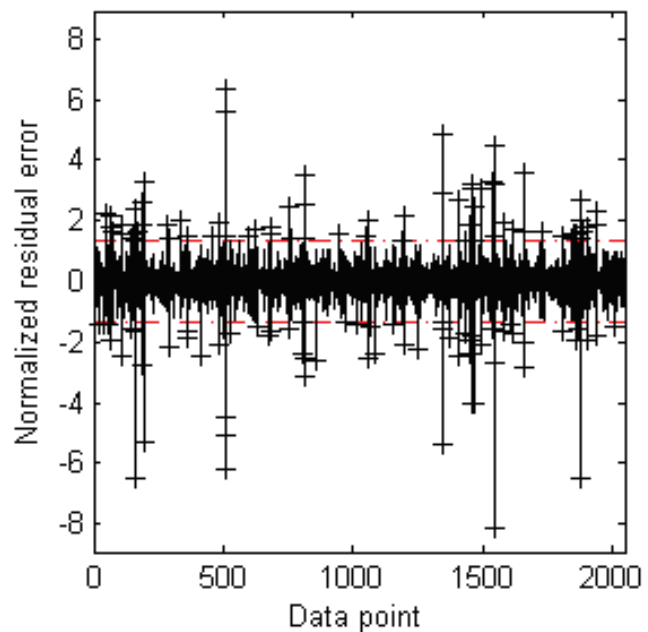

Figure 65: The X-Bar control plot of the mean of the grouped AR(5) residual errors (Channel 5). 
X-Bar Control Chart - State\#13

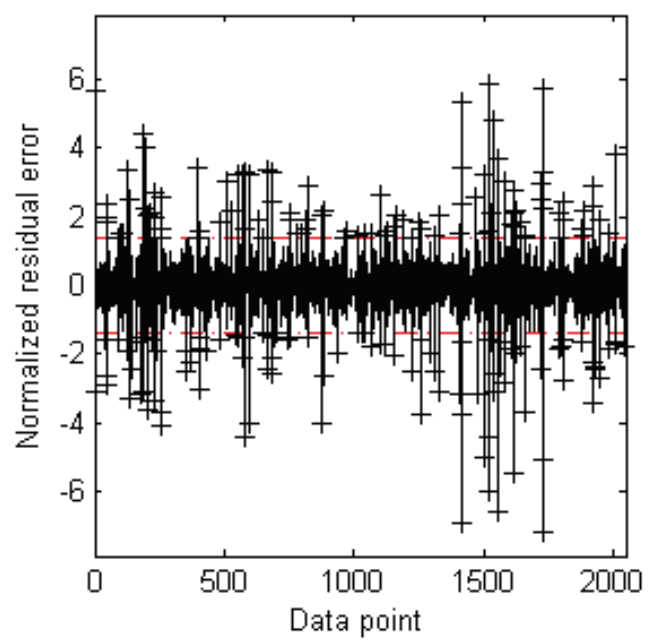

X-Bar Control Chart - State\#15

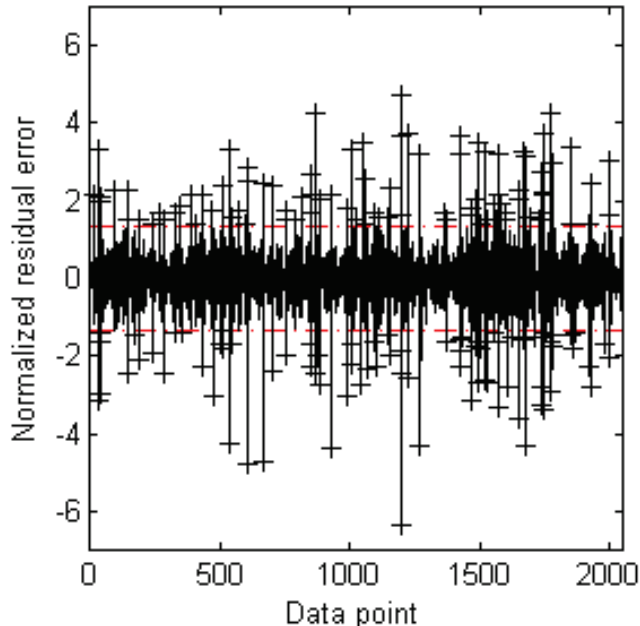

X-Bar Control Chart - State\#17

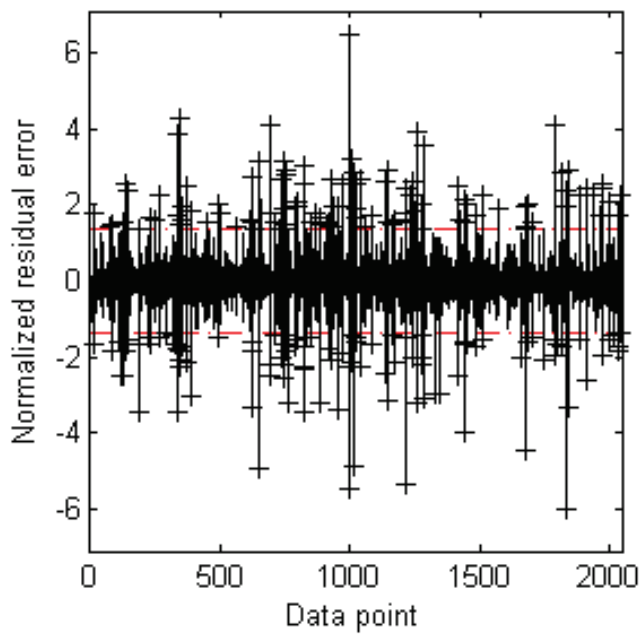

X-Bar Control Chart - State\#14

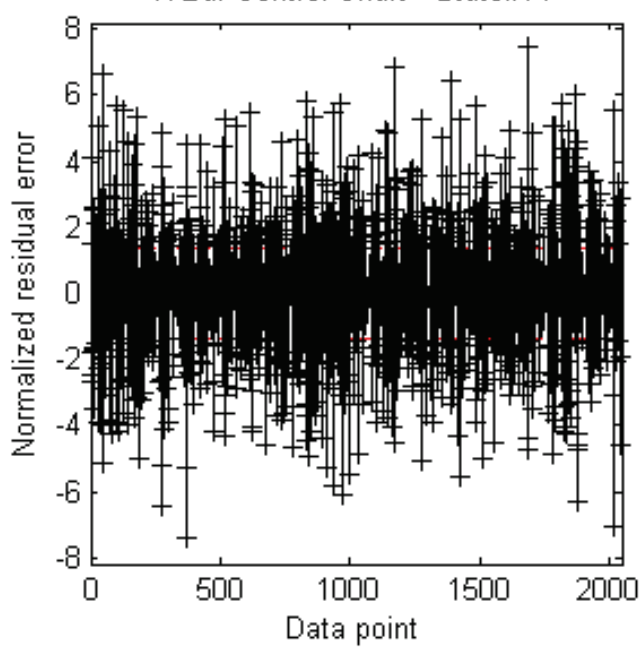

X-Bar Control Chart - State\#16

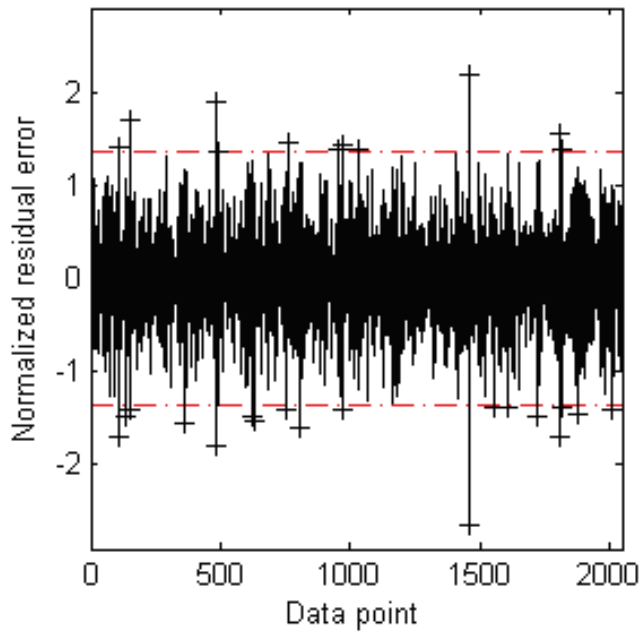

Figure 66: The X-Bar control plot of the mean of the grouped AR(5) residual errors (Channel 5). 

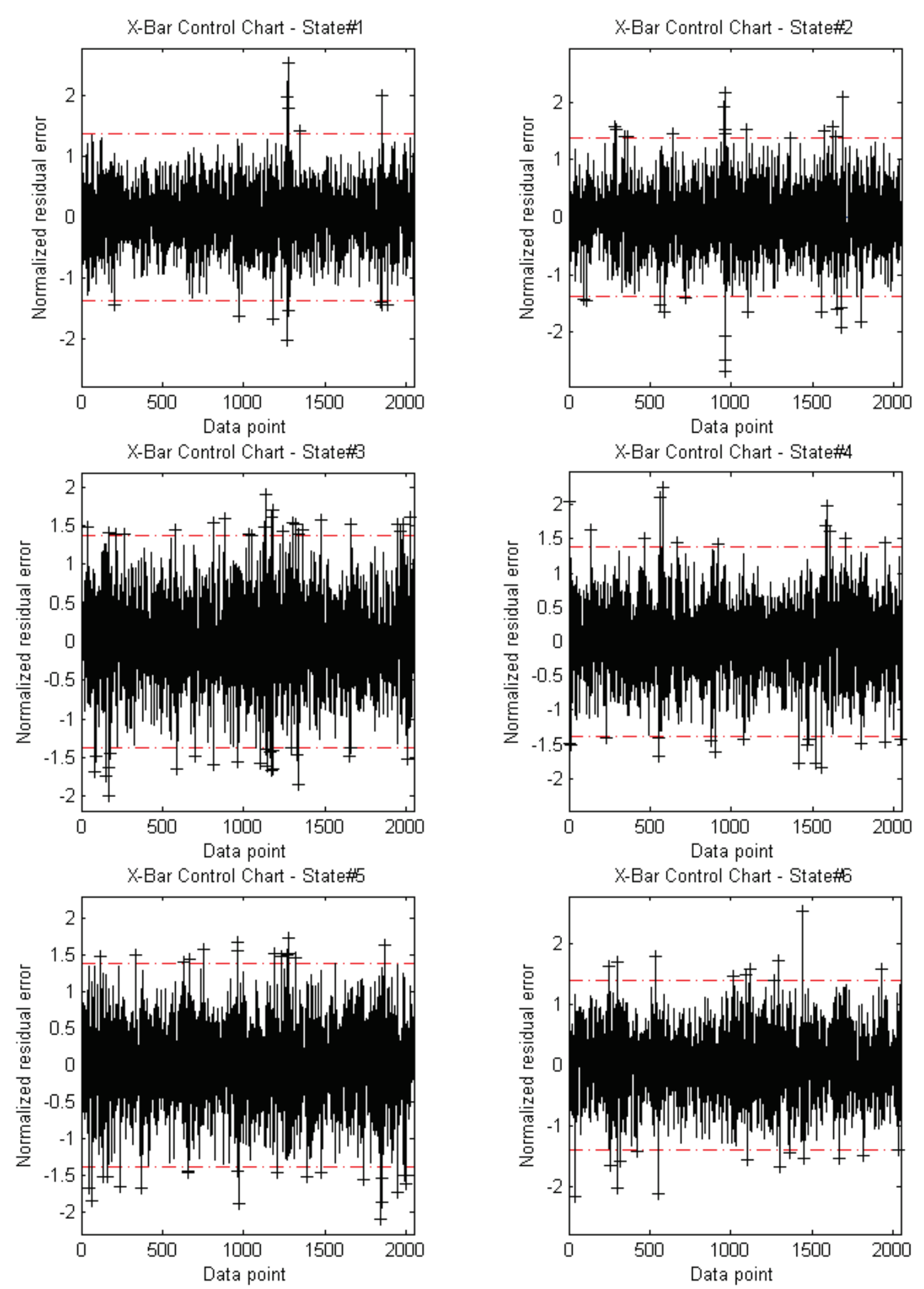

Figure 67: The X-Bar control plot of the mean of the grouped AR(30) residual errors (Channel 5). 
X-Bar Control Chart - State\#

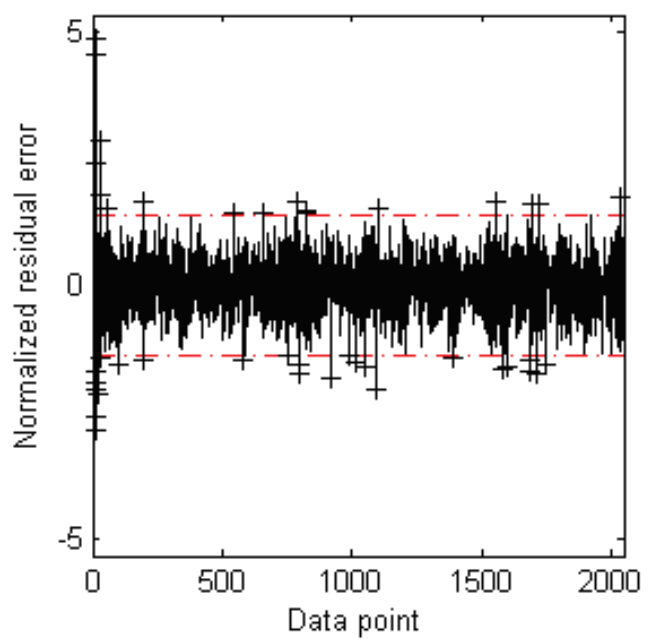

X-Bar Control Chart - State\#

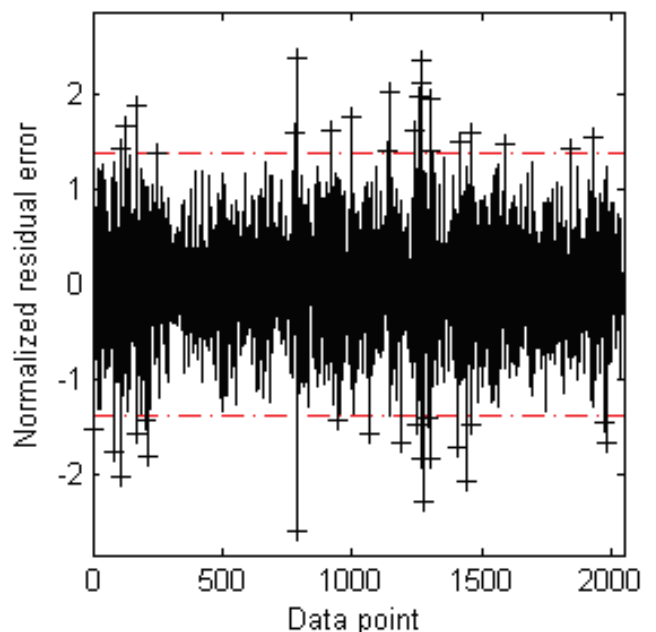

X-Bar Control Chart - State\#1

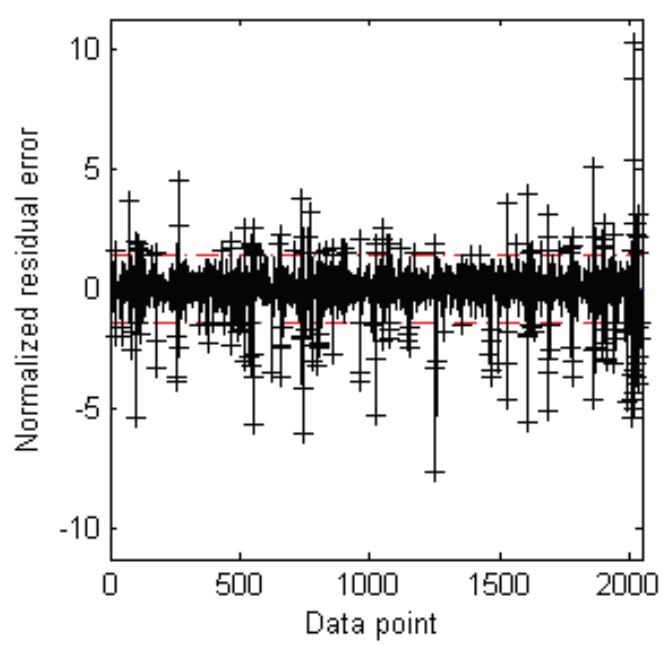

X-Bar Control Chart - State形

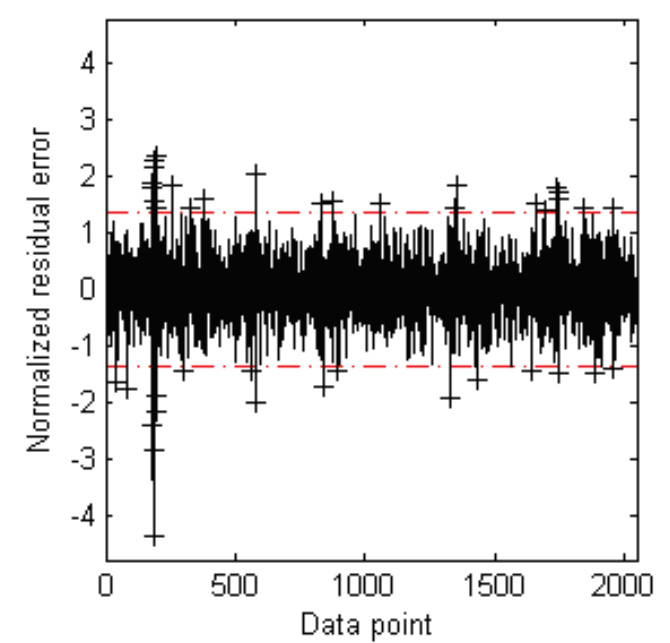

X-Bar Control Chart - State\#10

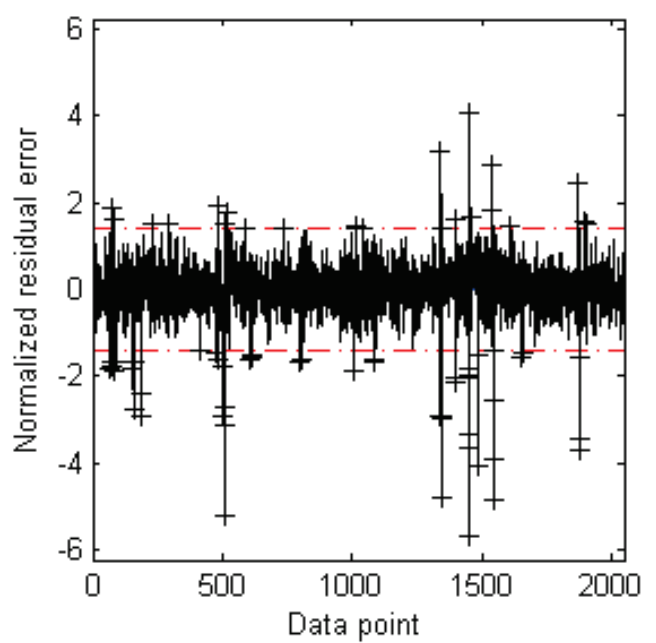

X-Bar Control Chart - State\#12

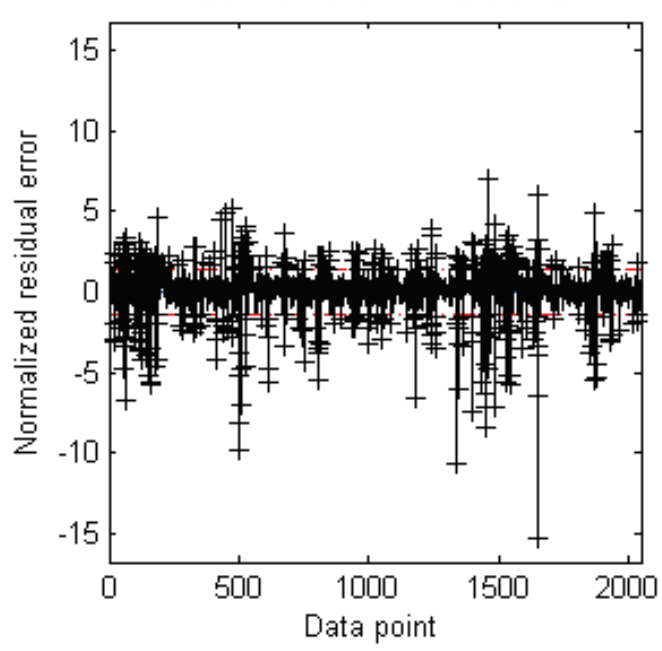

Figure 68: The X-Bar control plot of the mean of the grouped $A R(30)$ residual errors (Channel 5). 
X-Bar Control Chart - State\#13

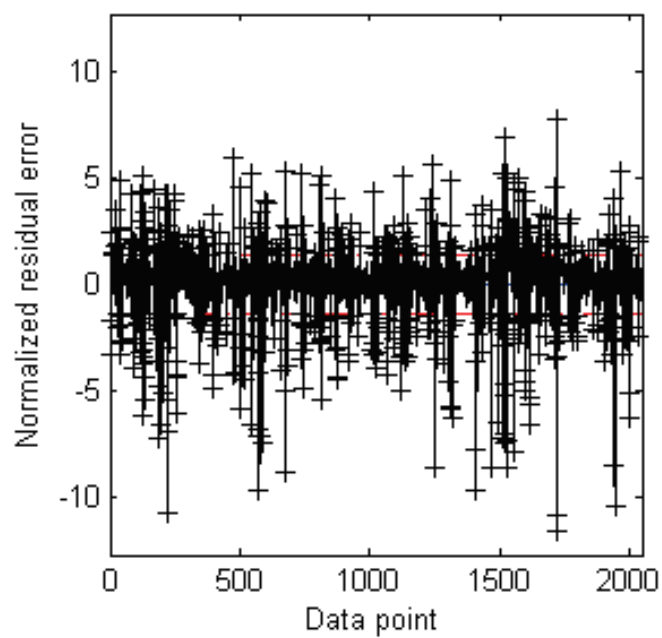

X-Bar Control Chart - State\#15

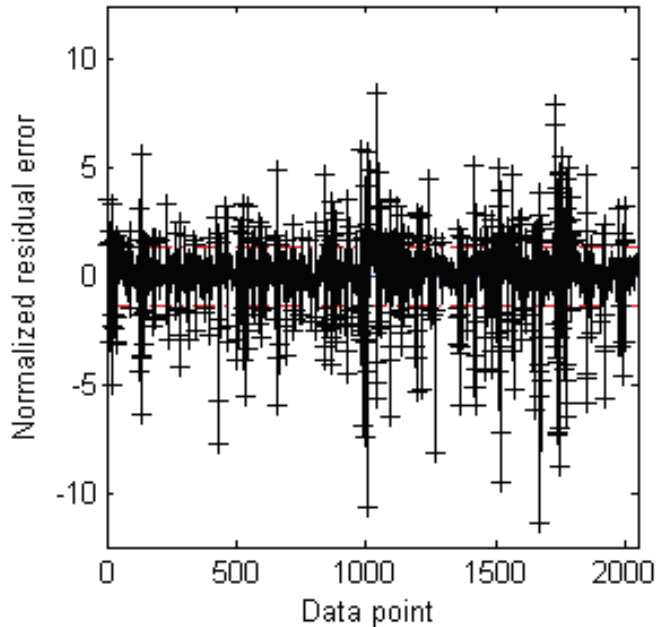

X-Bar Control Chart - State\#17

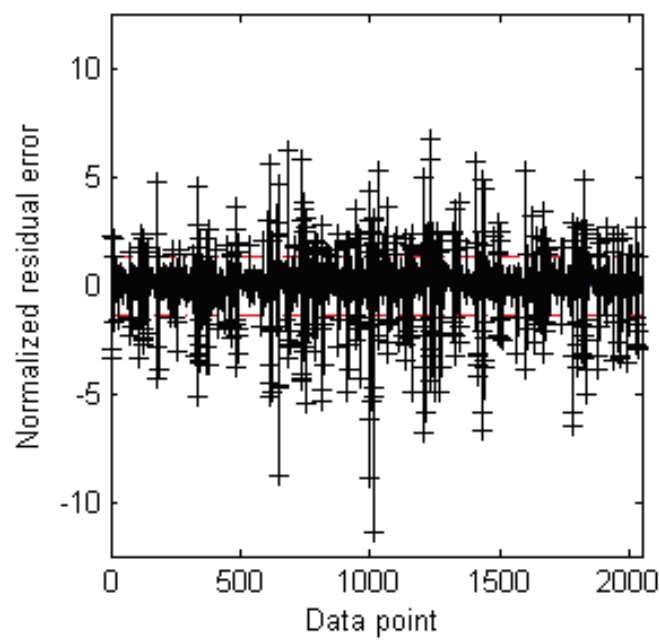

X-Bar Control Chart - State\#14
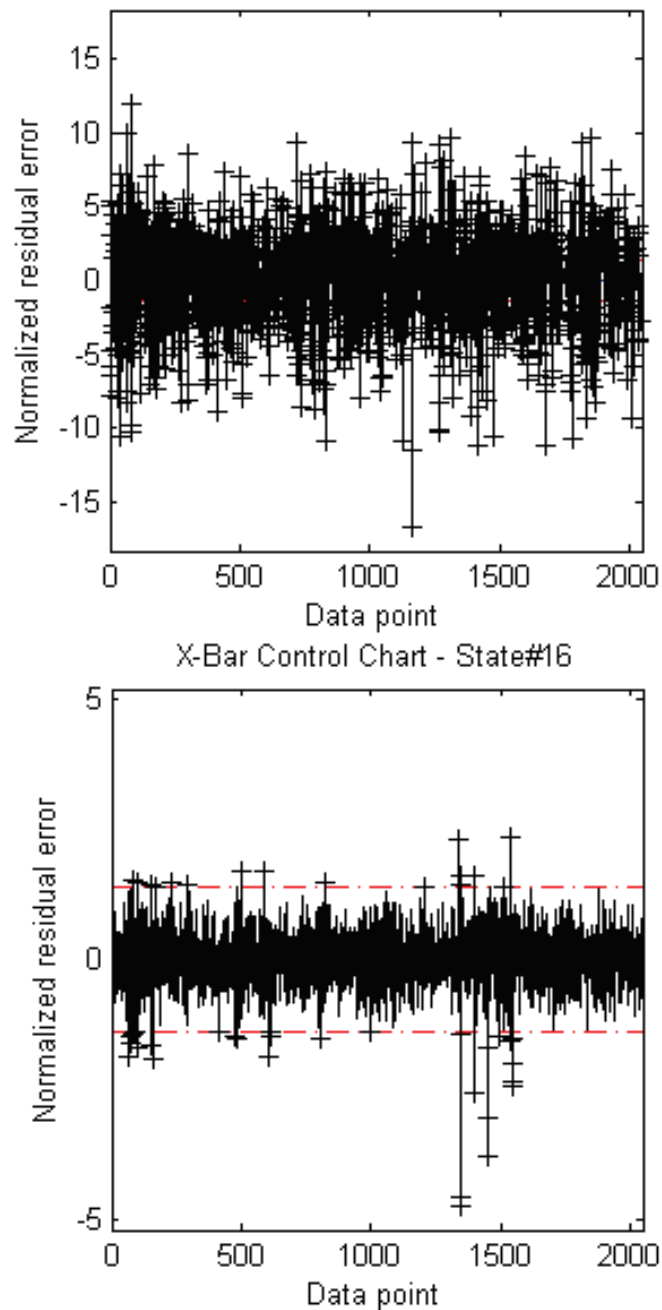

Figure 69: The X-Bar control plot of the mean of the grouped AR(30) residual errors (Channel 5). 
(a) Number of outliers associated with the AR(5) model

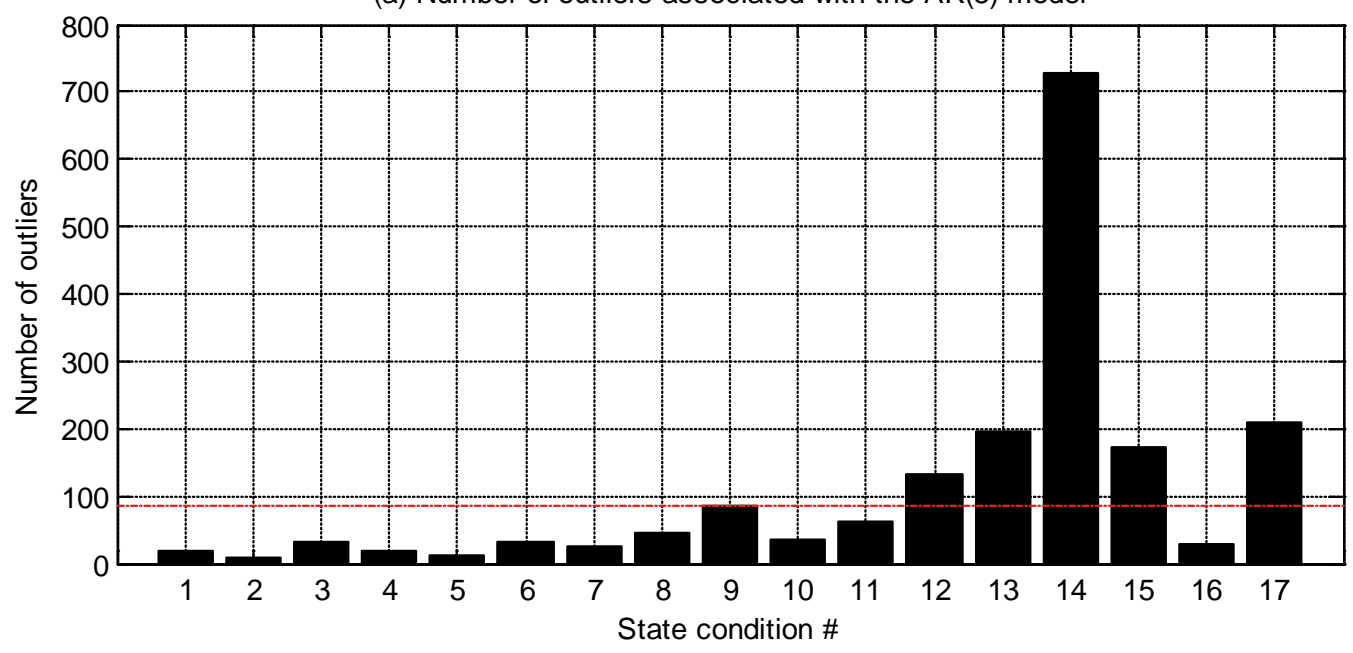

(b) Number of outliers associated with the AR(30) model

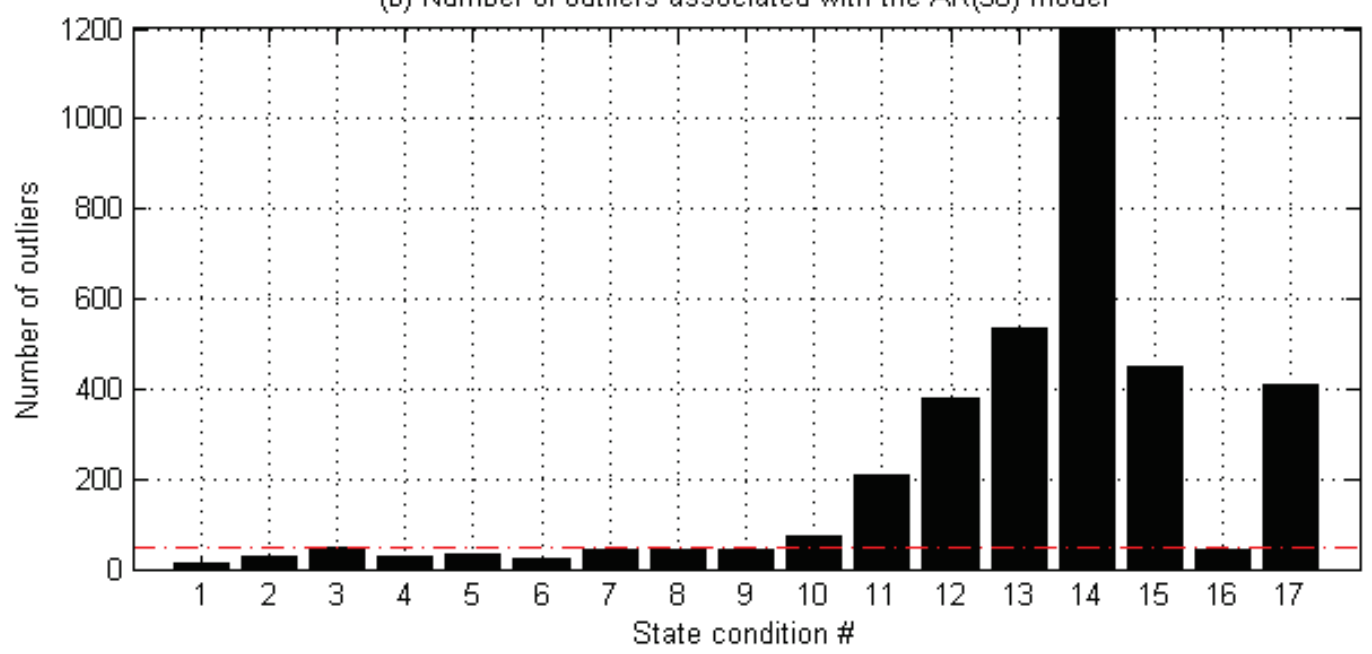

Figure 70: Number of outliers falling outside the control limits. The horizontal dashed line corresponds to the maximum number of outliers found in the undamaged states (Channel 5).

\subsubsection{Conclusions}

The Shewhart X-bar control charts were employed in order to control the mean value of the residual-error time histories from all state conditions. Results from this analysis showed that, in general, the number of outliers beyond the control limits increase for the damaged states even when they are affected by the operational and environmental variations. Also, the influence of model order on this classification technique was examined. More consistent results were obtained with the grouped AR(30) residual errors.

The control charts showed that SPC techniques might be used to detect the existence of anomalies in the acceleration responses. When a considerable number of data points fall outside the control limits, the process is considered out of control, and in this case, loss of control is assumed to be caused by anomalies. With such indications of damage, a next step might be to launch a full investigation, for example, through visual inspections or local off-line nondestructive evaluation, to identify the cause of such anomalies. 
Notice that there is still the possibility of all the points falling within the control limits, but the process is not in control. This is the case when the points exhibit some sort of systematic behavior, i.e., data points assume a nonrandom pattern. There are several common nonrandom patterns that indicate the process is out of control as documents in Montgomery (1997).

A problem that has often confronted the researches using control charts is when to consider recomputing the control limits. Initially, the control limits need to be established assuming that both the structure and the SHM system are undamaged. However, because of normal aging of the structures, one should evaluate the need to recalculate the control limits every time a statistically significant sign of instability is detected.

\subsection{Cluster Analysis}

Data clustering is a common statistical data-analysis technique that is used in many fields, such as machine learning, data mining, and pattern recognition. Data clustering is the classification of objects (variables) into different clusters (groups), so that the data in each cluster share some common underlying similarity (e.g., Euclidean distance).

There are many kinds of clustering (MATLAB, 2008), such as Hierarchical Clustering, which is a way to divide the data into different clusters by creating a cluster tree, as shown in Figure 71. In this process, clusters at one level are joined at the next higher level by progressively merging clusters. The higher the level in the tree, the smaller number of clusters.

In this study, cluster analysis is applied in order to discriminate the undamaged and damaged state conditions into two groups. Thus, the objects are the structural state conditions, and a binary classification in the form of two main clusters is assumed,

$$
\text { cluster }=\left\{\begin{array}{c}
+1 \text { if Undamaged } \\
-1 \text { if Damaged }
\end{array}\right.
$$

i.e., one corresponds to the undamaged state conditions $(+1)$ and another corresponds to the damaged state conditions (-1).

Figure 72 plots the binary classification of all state conditions using the parameters of the $\mathrm{AR}(30)$ model as damage-sensitive features extracted from the times histories of Channel 5. The Euclidean distance is the metric used to determine the similarity between AR parameters vectors that are used to define the system states. As shown in the figure, this technique can correctly classify all the state conditions.

Figure 73 plots the hierarchical cluster tree, where the states are paired into binary clusters by proximity until a hierarchical tree is formed. One can clearly observe that until the clustering reaches the top of the tree, the states are progressively grouped into two main state condition clusters: undamaged states and damaged states. The height of the clusters above the origin represents the Euclidean distance between the centroids of two clusters.

In conclusion, cluster analysis is able to group the undamaged and damaged state conditions into two main clusters based on the underlying similarity of the AR(30) parameters as damaged-sensitive features. 


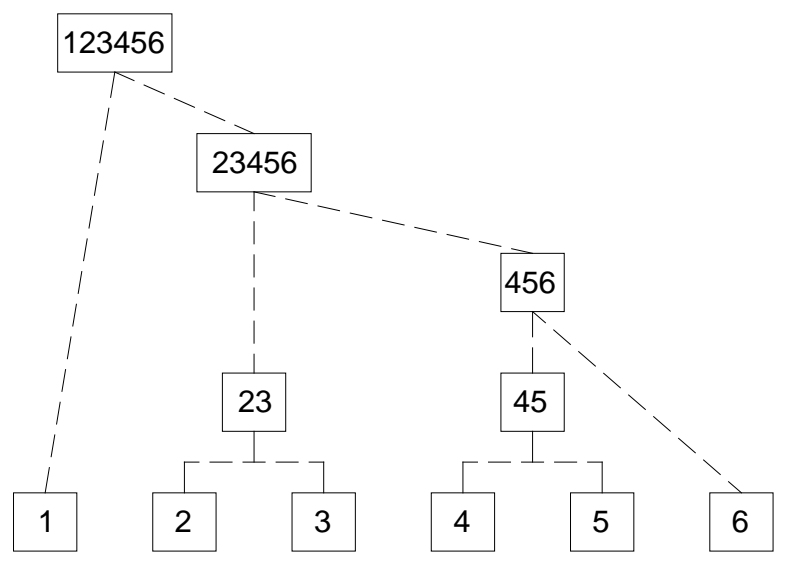

Figure 71: Traditional representation of the Hierarchical Clustering.

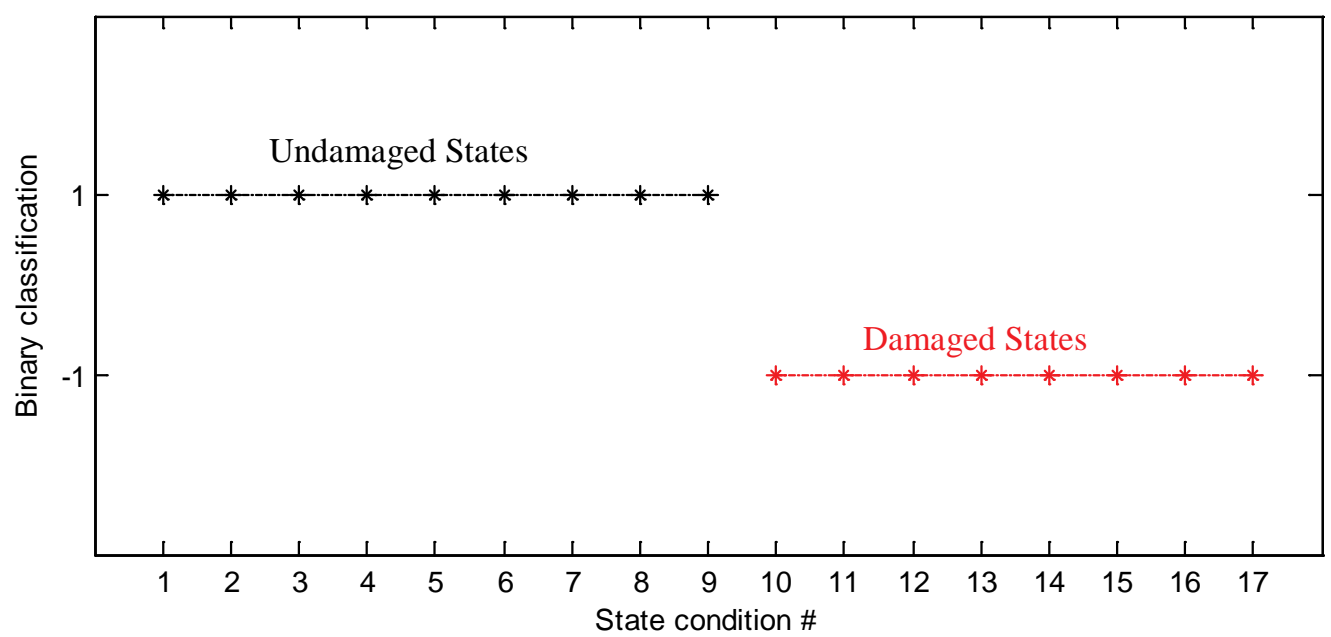

Figure 72: Feature classification assuming two clusters using AR(30) parameters (Channel 5).

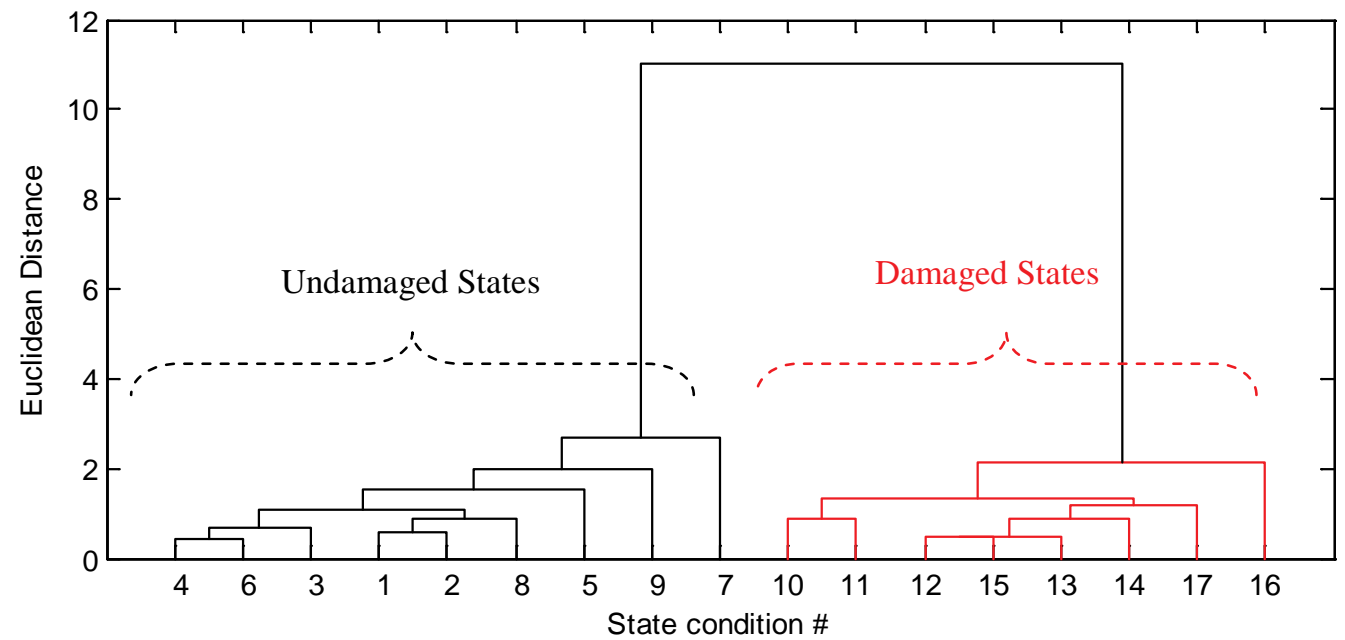

Figure 73: Cluster tree of the state conditions (Channel 5). 


\subsection{Factor Analysis}

In statistics, FA is a technique used to explain variability among observed random variables in terms of fewer unobserved random variables called factors. Thus FA attempts: (i) to reduce the number of variables, for instance to reduce two variables to one factor, and (ii) to explain the correlation between variables in terms of a small number of underlying factors not observed directly (e.g., temperature and humidity), in an effort to classify variables.

In the context of SHM, FA may be used as both data normalization (Kullaa, 2003; Kullaa, 2005) and a classifier technique. In this study, FA is used as a classifier technique to discriminate the undamaged and damaged state conditions.

Mathematically, the observed variables $X$ are assumed to be dependent on a linear combination of the common factors $\xi$, plus an error term $\varepsilon$ (unique factors) as follows:

$$
X=\Lambda \xi+\varepsilon
$$

where the coefficients stored in matrix $\Lambda$ are known as loadings.

Because each factor might affect several variables, they are known as common factors. A schematic model of this algorithm is shown in Figure 74.

Alternatively, the FA model can be specified by the correlation (or covariance) matrix, $\sum$, of the observed variables $X$, as follows:

$$
\sum=\Lambda \Lambda^{T}+\psi
$$

where the diagonal matrix, $\psi$, contains the specific variances. The specific variance is related to the independent random nature of each variable. In theory, a specific variance equal to 1 indicates no common factor component in that variable, but a specific variance of 0 indicates that the variable is entirely determined by common factors (MATLAB, 2008).

In order to demonstrate an application of this technique as a classifier, the seventeen state conditions are considered as variables, and the features are the AR(30) parameters extracted from one time history of Channel 5.

Assuming three common factors $\left(1^{\text {st }}, 2^{\text {nd }}\right.$, and $\left.3^{\text {rd }}\right)$, the dependence of each variable on the factors is illustrated in Figure 75 where the factor loadings $\Lambda$ are the coordinates. The figure clearly discriminates all the undamaged and damaged state conditions. Based on the distance (or proximity) of the states to the axes (MATLAB, 2008), one can reasonably conclude that the first factor axis represents the undamaged state conditions, and the second factor axis partially represents the damaged state conditions. Thus, the figure suggests the existence of an unobserved underlying variable common in all damaged state conditions. Although the damaged state conditions are influenced mostly by the second factor, they have nonnegligible components on the first and third axes.

In order to measure the level of independence of each state condition, Figure 76 shows the computed specific variance for each state. As stated above, a specific variance equal to 1 indicates no common factor component in that state condition, but a specific variance of 0 indicates that the state condition is entirely determined by common factors. In the figure, the damaged state conditions seem to assume higher specific variance; however, there are some exceptions. These results suggest that the nonlinearities introduce some random behavior into the damaged states and reduce the level of dependence between state conditions. 
In conclusion, the FA technique is able to discriminate all damaged state conditions from undamaged state conditions with a high level of certainty. It is able to identify unobserved underlying variables common in all damaged state conditions that drive the changes from the normal condition.

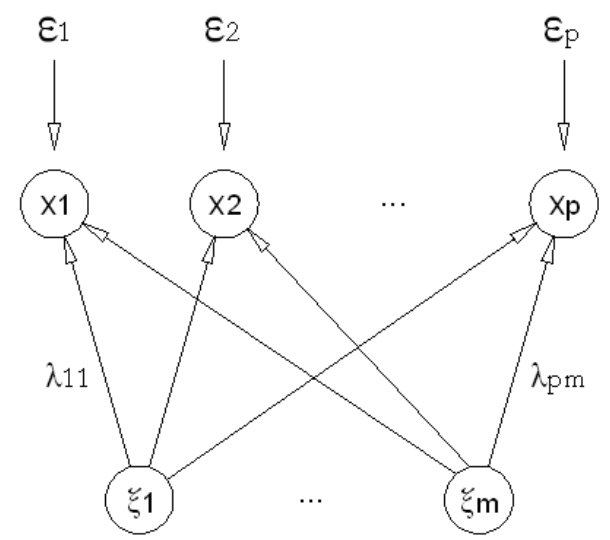

Figure 74: FA schematic model (Kullaa, 2003).

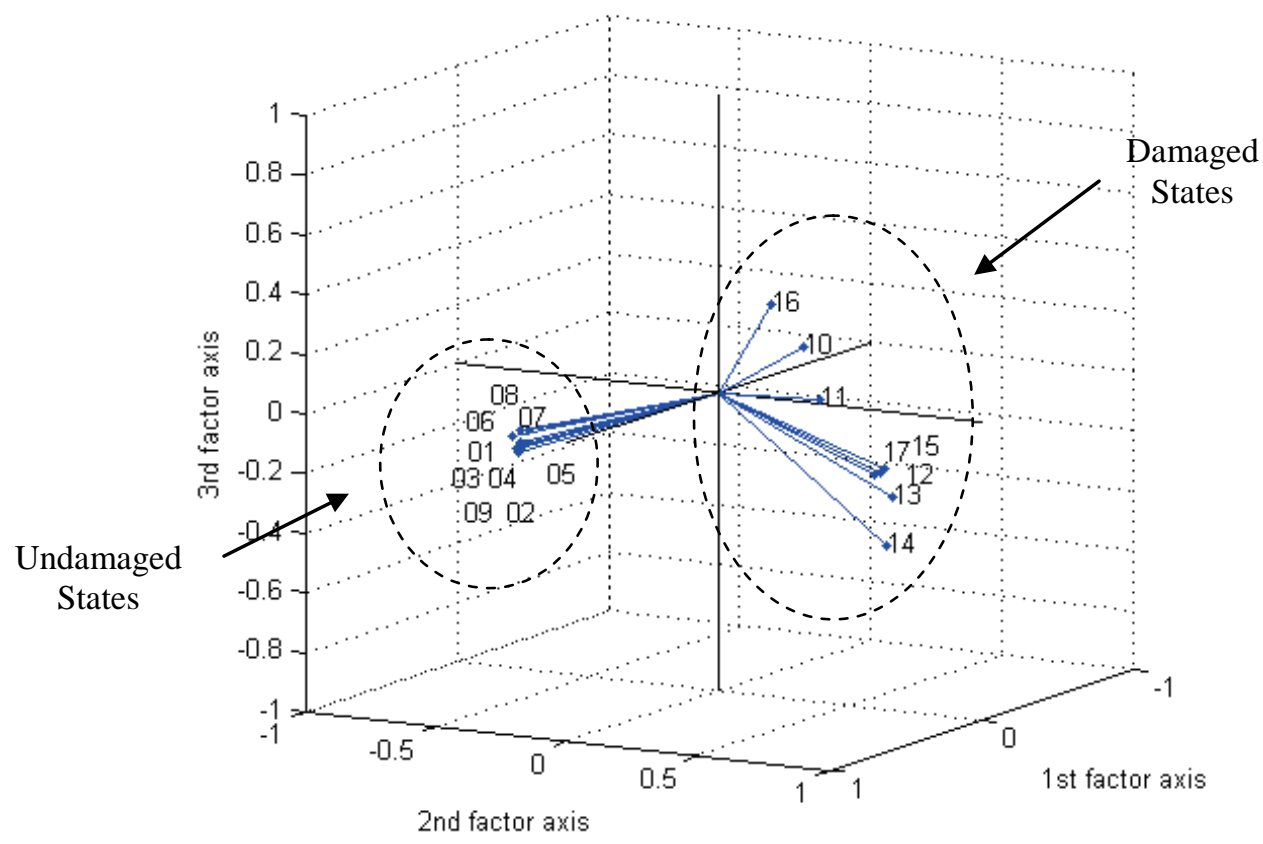

Figure 75: Factor loadings of each state condition (Channel 5). 


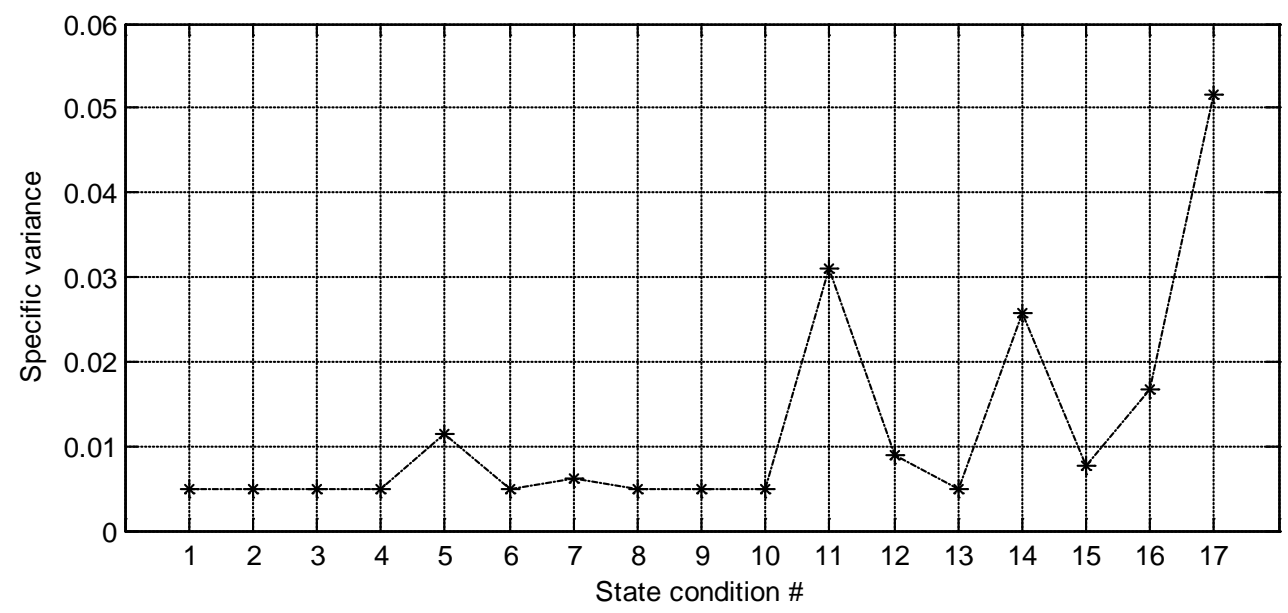

Figure 76: Specific variance by state condition (Channel 5).

\section{DATA NORMALIZATION FOR FEATURE CLASSIFICATION}

Inherent in the data acquisition, feature extraction, and statistical modelling portions of the SHM process, described in Section 1, is data normalization. In the context of SHM, data normalization is the process of separating changes in sensor reading caused by damage from those caused by varying operational and environmental conditions (Farrar et al., 2001).

As with the previous section, this section is associated with the feature classification portion of the damage-detection process. However, the authors decided to split the classification algorithms in two sections, because the algorithms applied in this section are performed in an unsupervised learning manner by first taking into account all the undamaged state conditions to train the algorithms. This procedure is currently defined as data normalization because it permits the algorithms to learn the underlying factors that influence the distribution of the undamaged state conditions.

Basically, in this section, each algorithm first uses training data composed of features from the normal condition of the structure, i.e., features from all the undamaged state conditions that include sources of variability (changing mass and stiffness) as previously described. This normalization procedure intends to make the classification algorithm output invariant to the sources of variability that influence the feature distribution. Therefore, when features from potentially damaged conditions are analyzed, it is expected that the classification algorithm will identify them as outliers even in the presence of operational and environment variability. As part of this process, threshold levels for outlier detection must be established that take into account the variability present in the data in an effort to reduce the number of false-negative and falsepositive indications of damage. For this purpose, three different algorithms are applied based on AANNs, Mahalanobis distance, and SVD technique. Note that different features (AR parameters and residual errors as well as FRFs) are used in order to enhance the flexibility of these damagedetection algorithms.

\subsection{Autoassociative Neural Network}

The AANN algorithm is employed as a novelty detection technique. Using features extracted from the time histories of the undamaged state conditions, the AANN is trained to 
characterize the underlying dependency of the identified features on the unobserved operational and environmental variations by treating this unobserved dependency as hidden intrinsic variables in the neural network. The architectural of the AANN consists of three hidden layers: the mapping layer, the bottleneck layer, and de-mapping layer. The number of nodes in each layer as well as the number of input features is problem specific. The name AANN comes from the fact that the target outputs are simply the inputs of the network.

Figure 77 shows the typical network architecture with $m$ damage-sensitive features, five nodes in both mapping and de-mapping layers, and two nodes in the bottleneck layer. This network is related to nonlinear principal components analysis (NLPCA). While PCA develops a mapping based on linear correlations among variables, NLPCA can find nonlinear correlations present among the features. More details about AANN can be found in the references (Kramer, 1991; Sohn et al., 2001; Jolliffe, 2004) .

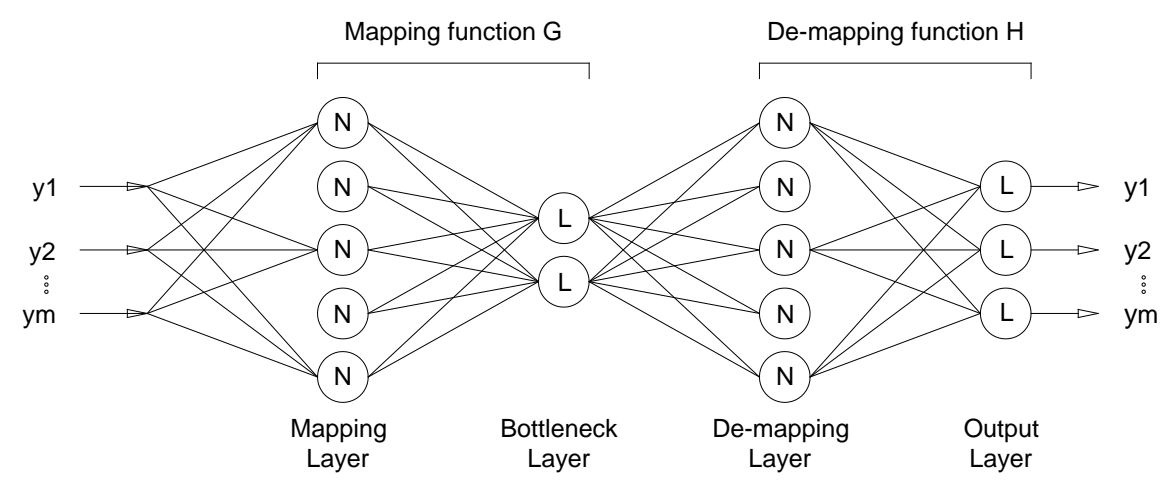

Figure 77: Network architecture of an AANN.

In the SHM field, the AANN can be used in two manners. First, the network can be trained to learn the correlations between features and, therefore, it can reveal the unobserved sources of variability that influence the structural response. This correlation is represented at the bottleneck output, where the number of nodes depends on the number of unobserved independent variables that influence the structural response. Second, assuming the network is trained to learn the correlations, the predicted errors at the output layer will grow when the features, which are fed to the network, come from a potentially damaged condition. Those residual errors are given by

$$
E=Y-\hat{Y}
$$

where $Y$ is the target vector, and $\hat{Y}$ is the output layer for each state. In order to establish a quantitative measure, a novelty index ( $N I$ ) can be adopted, which is defined to be the Euclidean distance between the targets and the outputs of the neural network. For a sample of a state condition $Y$ with $m$ features in the form of $y_{1}, \ldots, y_{m}$, the $N I$ is given by

$$
N I(Y)=\sqrt{\sum_{i=1}^{m}\left(y_{i}-\hat{y}_{i}\right)^{2}},
$$

where $y$ is the target feature and $\hat{y}$ is the output feature of the neural network. If the features are related to an undamaged state, $Y \approx \hat{Y}$ and $N I(Y) \approx 0$. On the other hand, if the features come 
from a damaged state, the neural network is not able to predict the targets, and the NI deviates from zero, thereby indicating an abnormal condition in the structure. Notice that the technique presented here is a mixture of two different learning algorithms, i.e., supervised learning is used to obtain the operational and environmental conditions' dependency, albeit without direct measure of these conditions, but unsupervised learning is used to detect damage.

In order to illustrate what the unobserved variable at the bottleneck output looks like, Figure 78 shows measured structural strain-time histories (Signal 1 and 2) at one deck cross section and the measured ambient temperature. The data were collected from the pedestrian bridge of Viana do Castelo during its normal operation (Figueiredo et al., 2007). It is assumed that the structural response is mainly influenced by the daily temperature variation. An AANN algorithm, with two inputs and two outputs, and one node in the bottleneck layer, is used to estimate the underlying unobserved variable. In the same figure, one can see the resulting bottleneck output that is seen to be related to the temperature. Notice that the measured ambient temperature is only a reference temperature variable because the temperature at several locations on the cross-section are not necessarily the same.

In order to reduce the computation effort, the AANN algorithm is trained using the AR(5) parameters as features. The training data matrix is composed of AR parameters from two time histories (Channel 5) of each undamaged state condition $(n=2 \times 9$ state conditions), i.e., the baseline condition (State \#1) state and states with simulated operational and environmental variations (States \#2-\#9). As a consequence, the training data matrix has a dimension of $5 \times 18$ ( $m=5$ and $n=18$ ). The features were scaled so that the data set ranges from -1 to 1 . The dimension of the mapping and demapping layers were fixed according to Kramer (1991). Therefore, the network is built up with a feed-forward neural network to perform the mapping and demapping, where the network outputs are simply the reproduction of the network inputs. The network has 10 nodes in each mapping and demapping layer and two nodes in the bottleneck layer. The nodes in the bottleneck layer intend to represent the two underlying parameters driving the changes in the features: the changes in stiffness and mass. A Levenberg-Marquardt back-propagation algorithm was used to train the network. Because it is not guaranteed to get the same sensitivity each training iteration, several trainings with different initial conditions were performed for that architecture to ensure that the global minimum was achieved. Features from one time history of each state condition, that were not used during the training, were subsequently used in order to validate the algorithm.

Figure 79 indicates that the residual errors of the AANN, given by Eq. (42), grow when the features of damaged states are fed to the network. The vertical dotted lines in the figure separate the state conditions (each segment is of 5 points). From a qualitative visualization, it seems to be unclear if State \#16 comes from an undamaged or damaged state condition. However, the novelty indices given by Eq. (43), and plotted in Figure 80, give a better discrimination of all state conditions. The horizontal threshold line is set based on the maximum magnitude for the undamaged state conditions. 


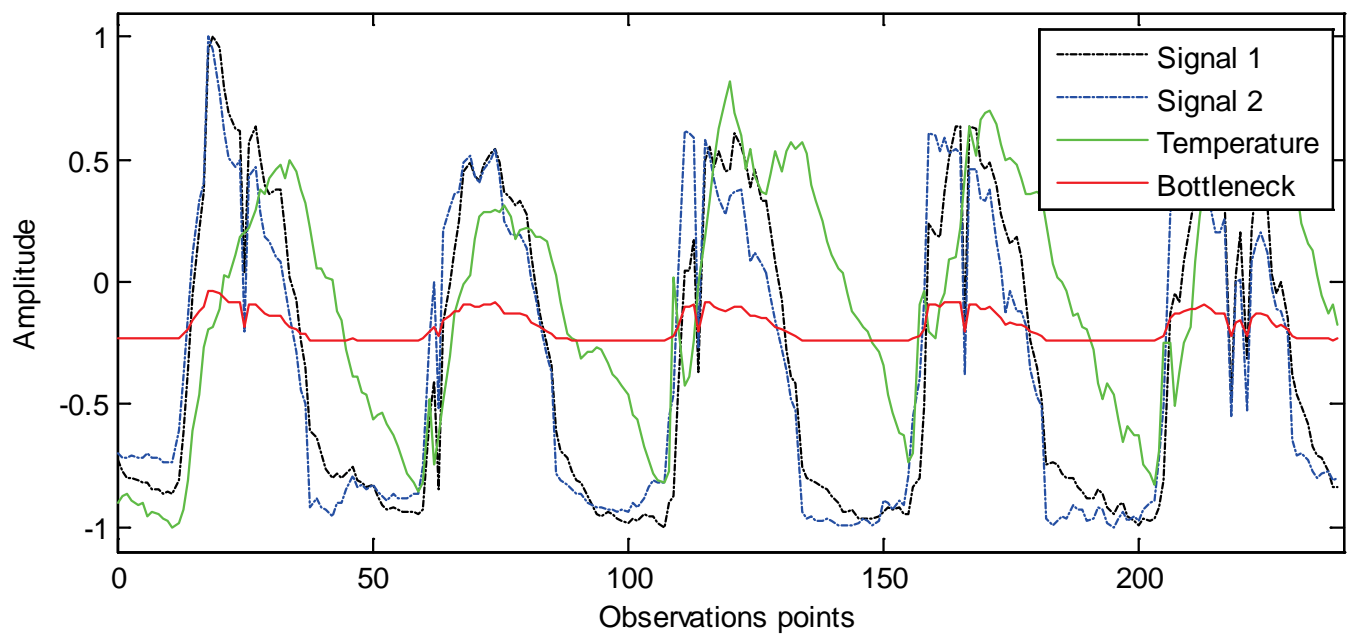

Figure 78: $\quad$ Output in the bottleneck layer and measured data (Signals 1 and 2, Temperature).

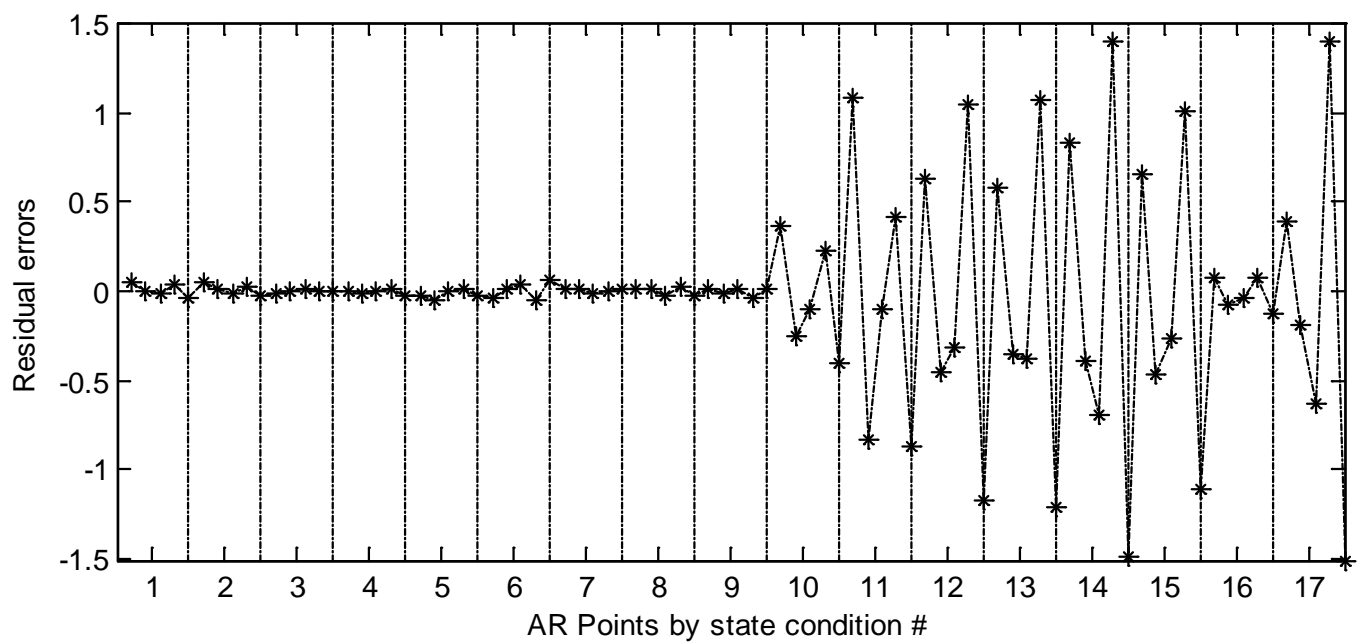

Figure 79: $\quad$ Residual errors using the AR(5) model parameters estimated at Channel 5.

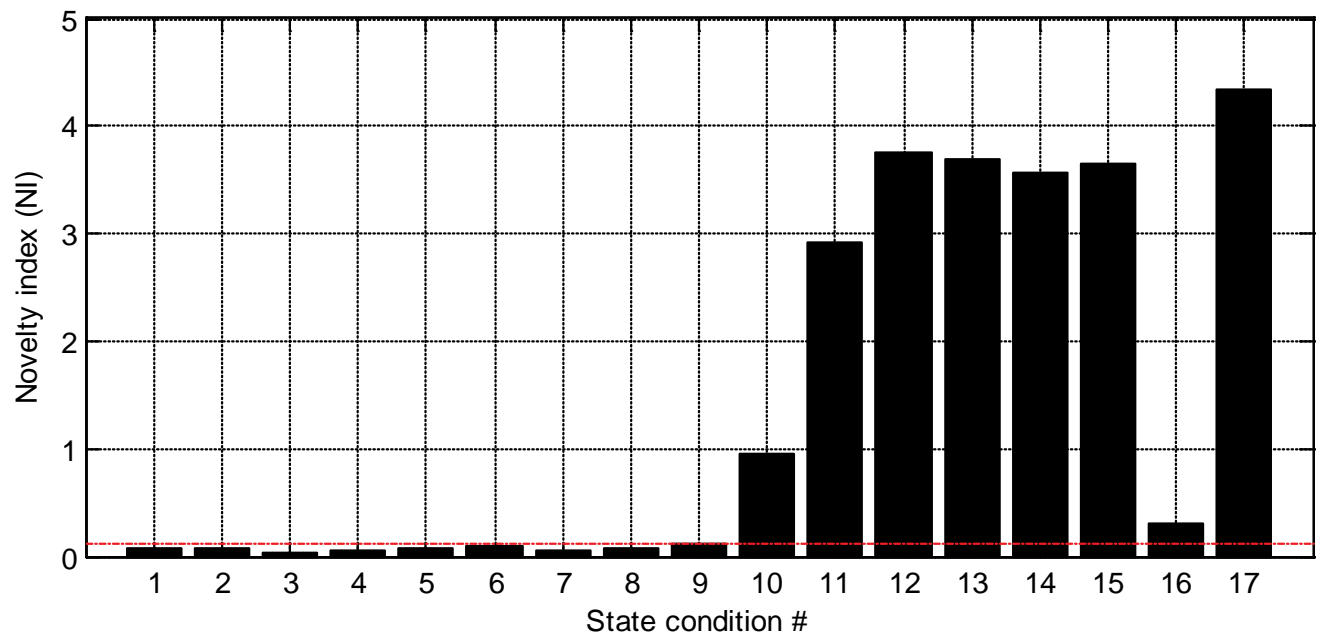

Figure 80: $\quad$ Novelty indices using the AR(5) model parameters estimated at Channel 5. 
In conclusion, the AANN algorithm was shown to discriminate all damaged and undamaged state conditions, when two nodes were used in the bottleneck layer to account for operational and environmental variations (intentionally introduced mass and stiffness changes in this case). Note that this procedure does not use any direct measure of these mass and stiffness changes. An underlying assumption is that the data sets used to train the network encompass this source of variability. If a new set of data were acquired under an environmental or operational condition not considered in the training, there is no guarantee that these will be properly classified. Also, in this study there was a priori knowledge regarding the number of operational and environmental parameters controlling the variability, and this information was used to choose the number of nodes in the bottleneck layer. Selection of the number of nodes in this bottleneck layer is critical to the performance of the AANN as a data normalization algorithm.

\subsection{Mahalanobis Distance}

The Mahalanobis distance is a distance measure used for multivariate statistics that can be used to identify and quantify outliers. The Mahalanobis distance differs from the Euclidean distance because it takes into account the correlation between the variables, and it does not depend on the scale of the observations.

Considering a group of $m p$-dimensional real-valued patterns belonging to $\mathfrak{R}^{p}$ with a multivariate mean vector $\mu=\left(\mu_{1}, \mu_{2}, \ldots, \mu_{p}\right)^{T}$ and covariance matrix, $\Sigma$, the Mahalanobis distance between that group and a new pattern $x=\left(x_{1}, x_{2}, \ldots, x_{p}\right)^{T}$ is defined as

$$
D=\sqrt{(x-\mu)^{T} \sum^{-1}(x-\mu)} .
$$

In the context of data normalization for feature classification, the mean vector $\mu$ and covariance matrix $\sum$ represent the normal operational condition and $x$ represents a potential damaged condition.

In this study, the normal condition of the structure is defined by using the AR model parameters from four time histories measured at Channel 5 and corresponding to each of the nine undamaged state conditions (States \#1-\#9). As a consequence, for the case of the AR(5) model, the mean vector and covariance matrix have a dimension of $5 \times 1$ and $5 \times 5$, respectively. In order to test the robustness of this data normalization method, Figure 81 and Figure 82 show the Mahalanobis squared distances calculated using Eq. (44) for AR(5) and AR(30) models, respectively. The test data are made up of one-time history for each state condition. Notice that the time histories used to define the normal condition were not used to test the method. The figures suggest that the effectiveness of the Mahalanobis distance as a damage indicator is a function of the AR model order. Furthermore, for the AR(30) model, the Mahalanobis distance provides a robust damage indicator that is almost invariant under the variability associated with the undamaged states. Note that in both figures, the Mahalanobis distances are presented in square units in order to enhance the differences between undamaged and damaged states.

Some authors (Worden and Manson, 2000) have used the Mahalanobis squared distance as a distance measure for multivariate statistics’ outlier detection. In this context, Eq. (44) should be rewritten as follows:

$$
D_{\zeta}^{2}=\left(x_{\zeta}-\bar{x}\right)^{T} \Sigma^{-1}\left(x_{\zeta}-\bar{x}\right)
$$


where the $x_{\zeta}$ is the potential outlier, $\bar{x}$ is the mean vector of the sample observations, and $\sum$ is the sample covariance matrix. The mean and covariance may be inclusive or exclusive (Worden and Manson, 2000). In this study, because the undamaged and damaged states are known $a$ priori, the method is developed in an exclusive manner, not using the data from the damaged condition in forming the estimates of the mean and covariance. As stated above, the normal operational condition of the structure is set up by the mean vector and covariance matrix, using only data from the undamaged state conditions.

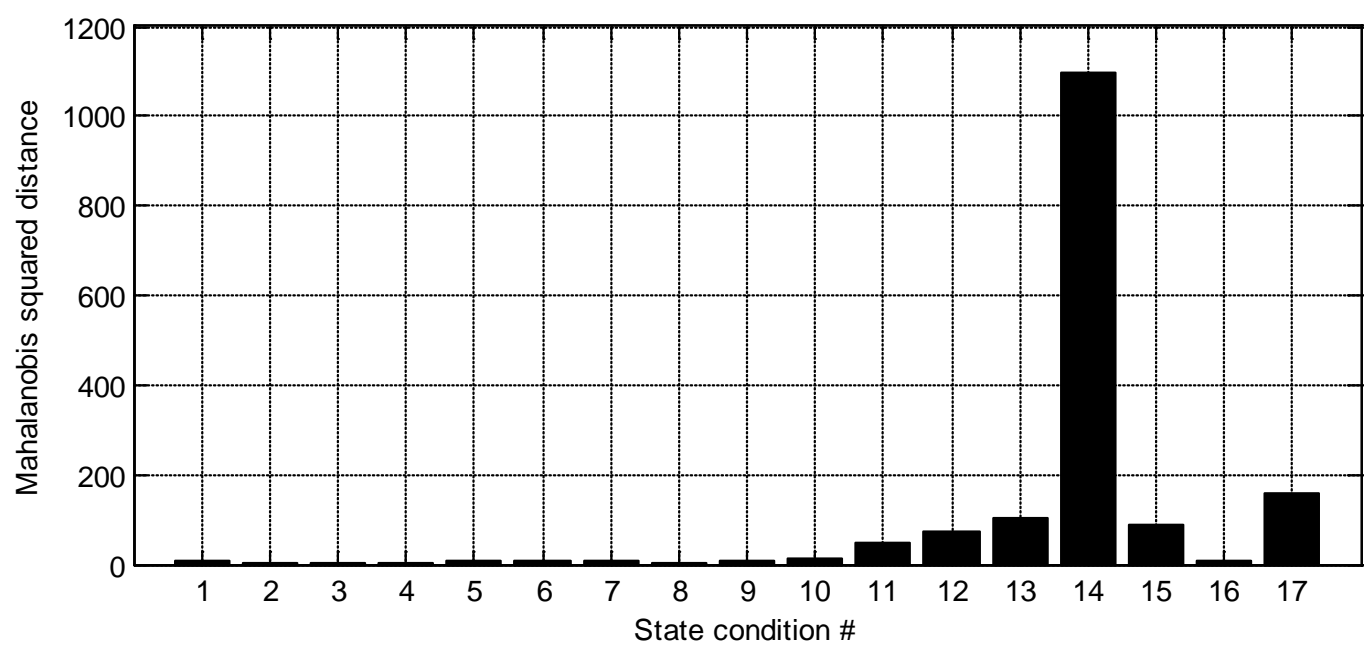

Figure 81: Mahalanobis squared distances using the AR(5) parameters (Channel 5).

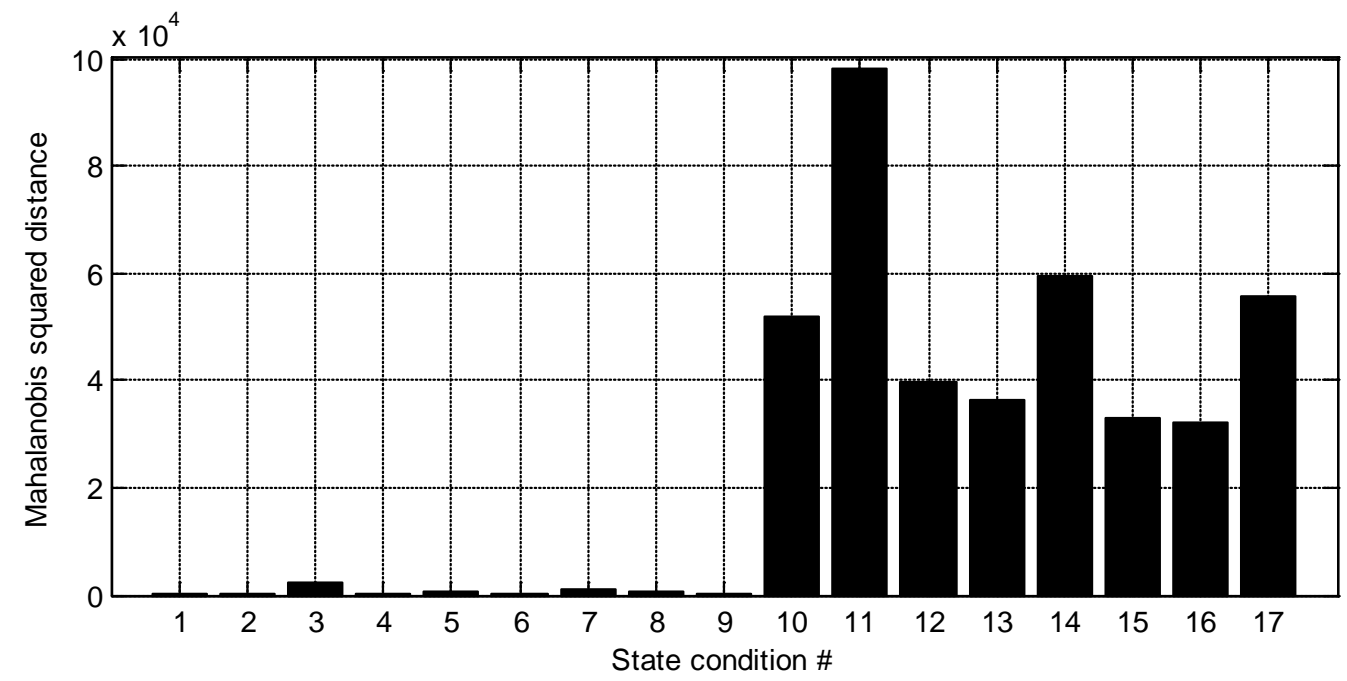

Figure 82: $\quad$ Mahalanobis squared distances using the AR(30) parameters (Channel 5). 
The exclusive Mahalanobis squared distances calculated using Eq. (45) are plotted, in concatenated form, in Figure 83 and Figure 84, based on the AR(5) and AR(30) parameters estimated from Channel 5, respectively. The results seem to discriminate the undamaged and damaged state conditions with a high level of reliability only for the AR(30) model.

However, the outliers plotted in Figure 84 have an unexpected pattern. Regardless of the mean vector and covariance matrix computed based on AR parameters from the undamaged states (training set), the magnitude of the outliers is much higher in the undamaged states (States \#1-\#9) than damaged states (States \#10-\#17). These results can be understood by examining the spatial distribution of the AR(30) parameters for each state. Recall that Figure 44 shows that the undamaged states assume a higher variance in the first principal component than the damaged state conditions. On the other hand, the centroid of the undamaged states given by $\bar{X}$ is very close to the centroid of the damaged states and, as a consequence, the Mahalanobis squared distance from a potential outlier is higher when it comes from undamaged states.

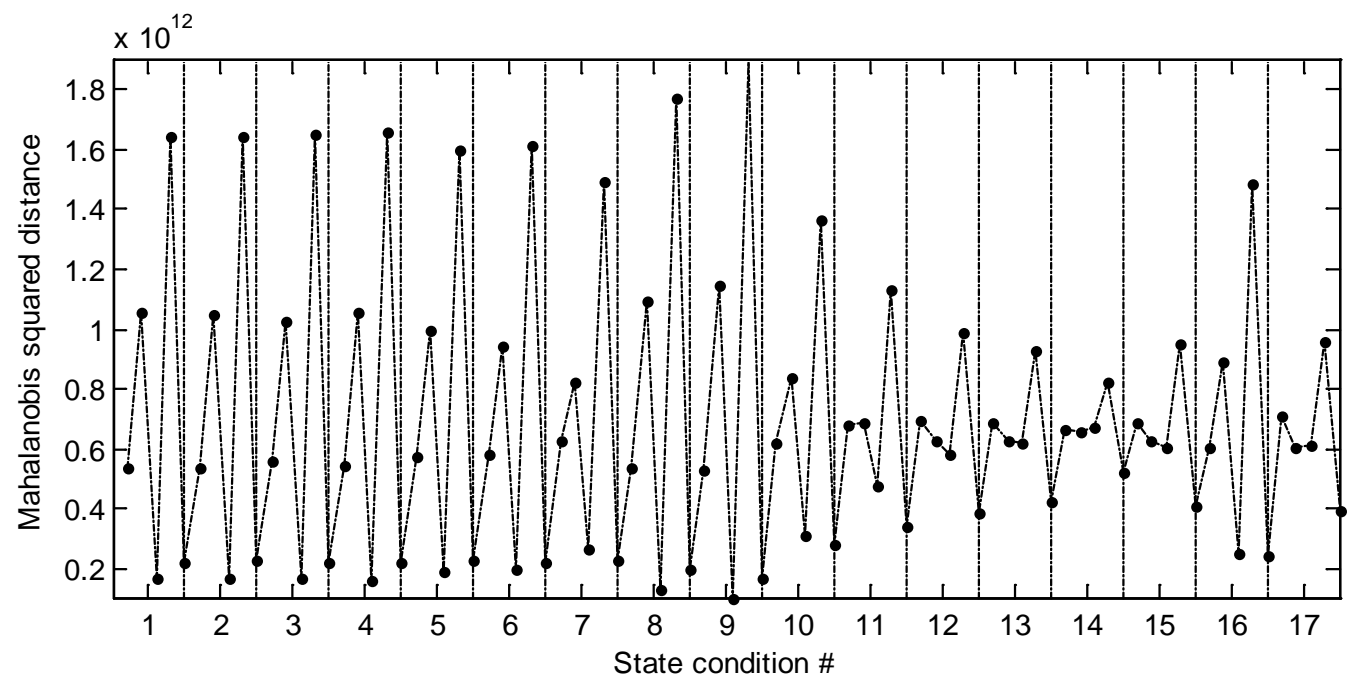

Figure 83: $\quad$ Mahalanobis squared distance for outlier detection using the AR(5) parameters estimated from Channel 5.

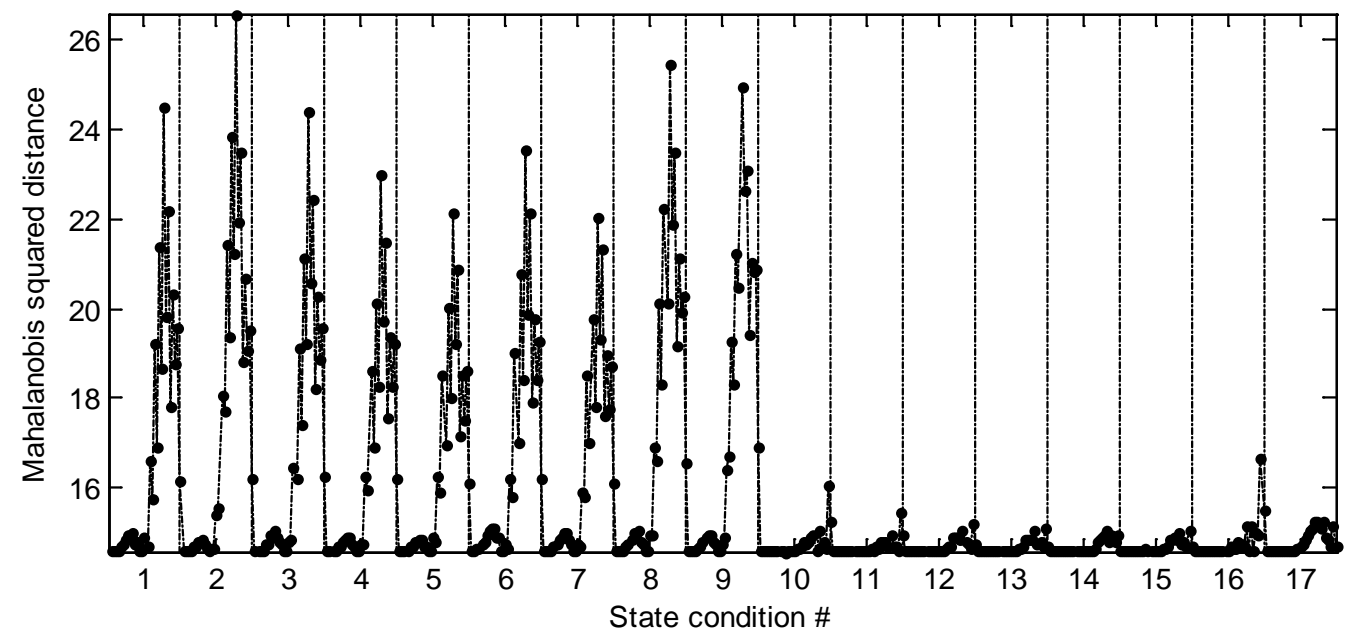

Figure 84: Mahalanobis squared distance for outlier detection using the AR(30) parameters estimated from Channel 5. 
Figure 85 and Figure 86 plot the Mahalanobis squared distances using the grouped residual errors from the different AR models as damage-sensitive features (see 4.2.1.2). Both figures show a higher density of outliers in the states with nonlinearities introduced by the bumper, with exception of State \#16. Furthermore, by simple visualization, the Mahalanobis squared distances also show which damaged state contributes most to the number and amplitude of the outliers.

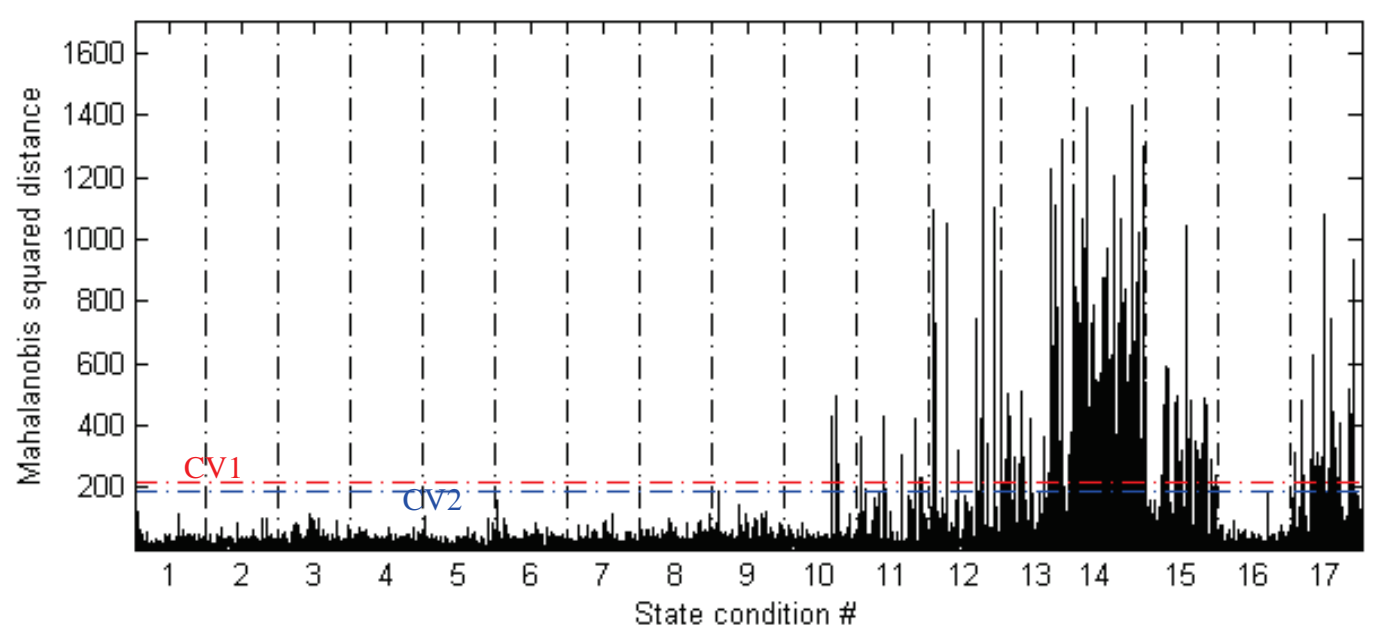

Figure 85: $\quad$ Mahalanobis squared distance for undamaged and damaged states using AR(5) grouped residual errors. The dash lines, CV1 and CV2, are the threshold lines.

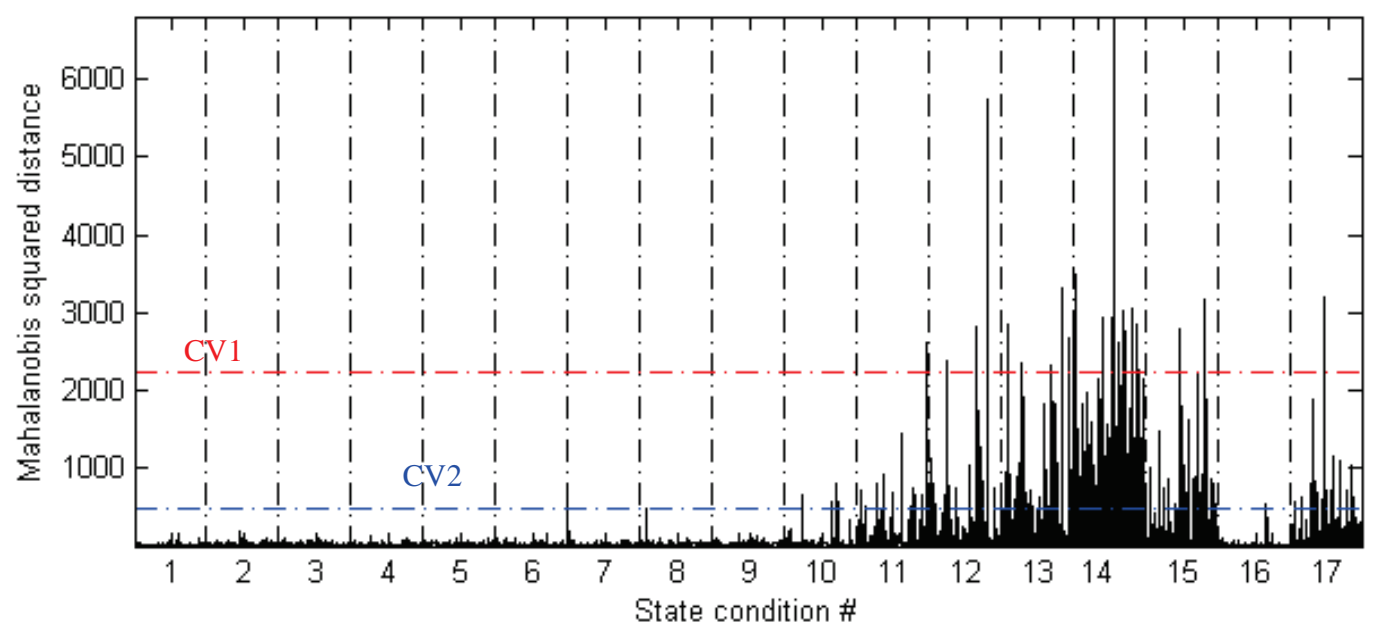

Figure 86: Mahalanobis squared distance for undamaged and damaged states using AR(30) grouped residual errors. The dash lines, CV1 and CV2, are the threshold lines. 
The establishment of threshold values for outlier detection is an important task in order to define a statistical separation between damaged and undamaged states. Some authors (Worden et al., 2000; Sohn et al., 2001) have proposed the Monte Carlo method to arrive at those values. However, in this study, another approach is presented based on data from all the undamaged states. The methodology used is summarized through the following steps:

i. $\quad$ Form a training-set matrix $(n \times p)$ with signals from the undamaged state conditions. Rows $n$ are the observations and the columns $p$ are the number of state conditions. The more known undamaged state conditions that are available, the more likely the Mahalanobis squared distance will be able to detect damage. In this case, the signals are the AR-grouped residual errors estimated from the time histories of Channel 5;

ii. Calculation of the mean vector $\bar{x}$ and covariance matrix $\sum$;

iii. Calculation of the Mahalanobis squared distances, $D_{\zeta}^{2}$, for all signals corresponding to each undamaged state condition;

iv. The threshold for outlier detection is defined by the critical value (CV) equal to the largest value of $D_{\zeta}^{2}$ calculated in step iii.

Figure 85 and Figure 86 show the Mahalanobis squared distances along with a horizontal threshold line defined by the critical values CV1. Taking into account the states with points falling beyond the threshold line, for the AR(5) and AR(30) model, the procedure outlined above gives one false-negative indication (State \#16) and two false-negative indications (States \#10 and \#16) of damage, respectively. The increase in the number of false indications for the higher order AR model is an indication that the $\mathrm{AR}(30)$ model is probably overfitting the original time histories.

In order to overcome the overfitting related limitation, the critical values CV2 shown in the same figures are estimated based on a confidence interval for the parameter of an exponential distribution. Thus, steps (iii) and (iv) outlined above are changed as follows. First, an exponential distribution is assumed that best fits in some sense the largest values $D_{\zeta}^{2}$ of each AR-grouped residual errors' series from all the undamaged state conditions (States \#1-\#9). Note that for Channel 5, each state condition has ten series. Thus, the exponential distribution has 90 data points. Then, the parameter of the exponential distribution that gives the highest likelihood is calculated, given the 90-point data. Finally, in order to discard the extreme values, the upper bound 99\% confidence interval for the parameter is assumed as the critical value. As shown in Figure 85 and Figure 86, the new threshold line improves the discrimination especially for the AR(30) model, where it is able to discriminate all the undamaged and damaged state conditions. Note that this procedure does not take into account the extreme values caused by the overfitting associated to the $\mathrm{AR}(30)$ model.

In conclusion, the reliability of the Mahalanobis distance in data normalization for feature classification was demonstrated to be a function of the type of damage-sensitive features used. When assuming the AR parameters as damage-sensitive features, the performance is sensitive to the number of AR parameters. In this case, the AR(30) model gives better results than the AR(5) model. On the other hand, using the AR grouped residual errors as damage-sensitive features together with the methodology proposed to define the threshold limits, the AR(5) model proved to perform better than the AR(30) model. However, it was demonstrated that by using confidence intervals, it is possible to improve the classification. This procedure is important for discarding extreme values that frequently are related to noise or singularities imposed, for instance by the acquisition system, that influence the threshold values. 


\subsection{Singular Value Decomposition}

Some authors have proposed a damage-detection method based on the SVD technique (Ruotolo et al., 1997; Ruotolo et al., 1999). This technique relies on the determination of the rank of a matrix. In the SHM context of data normalization for feature classification, the aim of this technique is to detect damage regardless of operational and environmental effects. A very brief summary of the approach will be given for completeness.

In linear algebra, the SVD is a factorization of a rectangular real or complex matrix $M$ defined by

$$
M=U \Lambda V^{H},
$$

where $U$ and $V$ are two orthogonal matrices, i.e., $U^{H} U=V^{H} V=I$, and $\Lambda$ contains the singular values of matrix $M$ along its diagonal line sorted in descending order. The superscript ${ }^{\mathrm{H}}$ denotes complex conjugate transposal.

In the context of damage detection, the matrix $M$ can be defined as a state matrix composed of characteristic vectors, $w_{i}$ and $w_{d}$, which represent a particular property of the structure, e.g., natural frequencies, mode shapes, and FRFs, where $w_{i}, i=1, \ldots, p$, is related to the normal operational condition $i$, and $w_{d}$ is related to the current condition that may be damaged or undamaged. For $p$ known normal operational conditions and one current condition, the state matrix $M$ can be defined as

$$
M=\left[W_{1}, \ldots, W_{p}, W_{d}\right] .
$$

By estimating the rank of the matrix $M$, if the characteristic vector $w_{d}$ comes from an undamaged structure, it is expected that the rank will not change and will be equal to $p$. By contrast, if $w_{d}$ comes from a damaged structure, the rank will be equal to $p+1$.

Theoretically, if $M$ is rank deficient, i.e., some undamaged state conditions are a linear combination of the others, some of the singular values should be zero. However, real-world data are not perfect and will often have measurement noise that can mask the state conditions, which would affect the rank of $M$. Thus, the presence of noise in the data introduces residuals in the singular values. In order to discriminate the higher singular values related to the state conditions from ones related to the noise, it is important to define threshold values.

In order to use the SVD technique to discriminate the undamaged and damaged state conditions, a three-step process is outlined as follows:

i. Calculate the singular values of the state matrix $M_{1}=\left[w_{1}^{n}, \ldots, w_{p}^{n}\right]$ with only undamaged state conditions. The more samples per state, the lesser the influence of noise. Rows $n$ are the observations and the columns are the number of state conditions $p$. This matrix intends to set the normal operational condition and, therefore, it defines the reference condition of the structure;

ii. Calculate the singular values of a state matrix $M_{2}=\left[w_{1}^{n}, \ldots, w_{p}^{n}, w_{d}^{n}\right]$ with the previous undamaged states plus one potential damaged state condition $w_{d}^{n}$; This matrix intends to test the actual state of the structure;

iii. Fit two independent curves to the previous singular values from $M_{1}$ and $M_{2}$; when the curves are plotted together, if the potential damaged state condition is effectively related to some source of variability in the structure not seen before, the curves should 
not overlap because it is assumed that the potential damaged state, $w_{d}^{n}$, is linearly independent of the other undamaged states.

The process described above is applied individually for Channels 2, 3, 4, and 5, using FRFs. These kind of functions are useful, because they take into account relationship between the acceleration responses and the excitation force applied to the structure and thus fully characterise the system's dynamic properties (Maia et al., 1997). Figure 87 plots the magnitude of the FRFs from Channel 4 for five state conditions (States \#1, \#3, \#7, \#14, \#17). Clearly, the FRFs from the damaged states show distinct changes at high frequencies. The portion of the FRFs below $20 \mathrm{~Hz}$ is related to noise, and therefore it is ignored, as explained in Section 2. As a consequence, each FRF has 3,088 data points. Notice that four channels are used in order to analyze the sensitivity of each accelerometer to detect nonlinearities introduced at the third story of the structure.

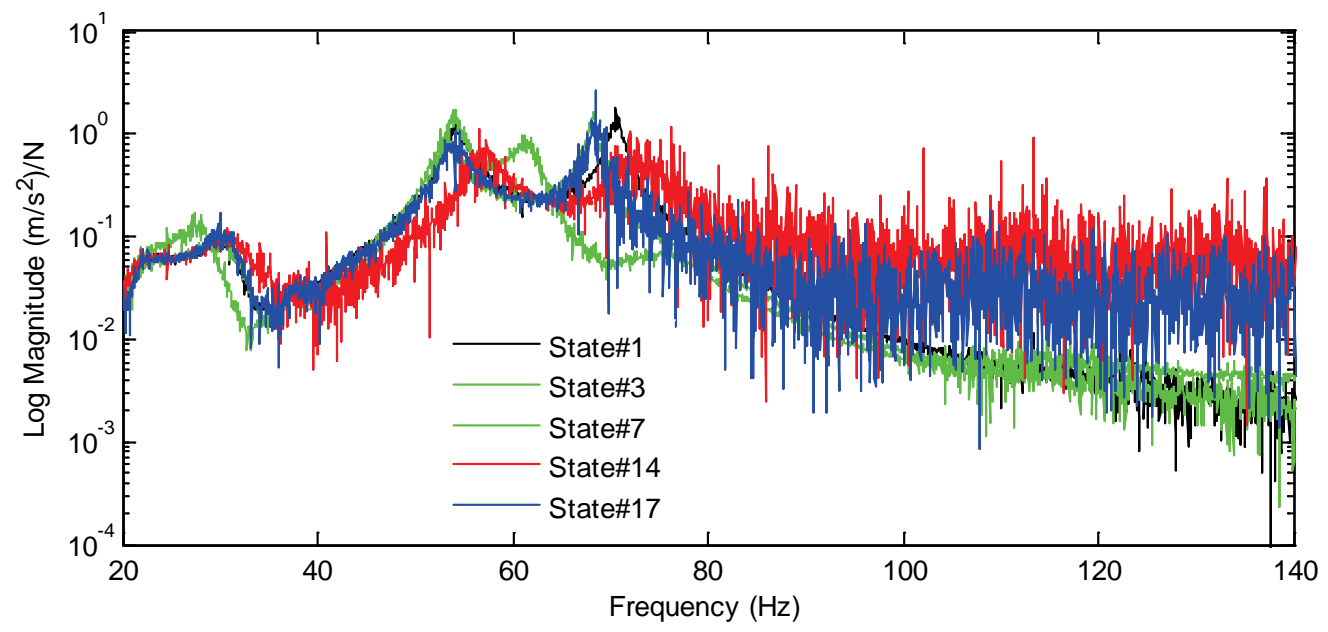

Figure 87: FRFs of five state conditions estimated at Channel 4.

For each channel, the normal operational condition state matrix $M_{1}$ is composed of two FRFs from time histories of each undamaged state condition (States \#1-\#9) in order to increase the redundancy and decrease the influence of noise in the measurements. Because the experimental data set contains nine known undamaged state conditions, $M_{1}$ is composed of eighteen characteristics vectors. As a consequence, $M_{1}$ has a dimension of $3088 \times 18$. The state matrix $M_{2}$ is composed of the same characteristics vectors of $M_{1}$ plus one characteristic vector $w_{d}$ related to a potential damaged state, namely from States $\# 1, \# 3$, \#7, \#14, and \#17. As a consequence, in each run, $M_{2}$ has a dimension of $3088 \times 19$. Note that the characteristic vector $w_{d}$ varies in order to examine the capabilities of this method to detect the different damage conditions. It follows that one should expect deviations from the normal condition only when $W_{d}$ is composed of FRFs from States \#14 and \#17.

Figure 88, Figure 89, Figure 90, Figure 91, and Figure 92 plot the first fitted 15 singular values (Channels 2-5) when $w_{d}$ is composed of FRFs from States \#1, \#3, \#7, \#14, and \#17, respectively. In the figures, the "Reference condition" represents the fitted singular values from $M_{1}$ and the "Test condition" represents the fitted singular values from $M_{2}$. Notice that for the undamaged state conditions, the characteristic vector $W_{d}$ is not used in $M_{1}$ to establish the 
normal condition. As expected, the figures indicate slight deviations from the normal condition when $w_{d}$ is composed of FRFs from damaged state conditions (States \#14 and \#17). Moreover, for those states, even though this technique seems to be sensitive to detect damage across all accelerometers, the magnitude of the deviation decreases for the accelerometers located far from the source of damage.

In conclusion, the method presented based on the SVD technique has revealed potential for data normalization when well-known undamaged state conditions exist. The more undamaged conditions are available, the more feasible the method can be. However, the analysis carried out was only qualitative and therefore there is a need to establish threshold values that can be useful to evaluate if statistically significant changes have occurred in the singular values.
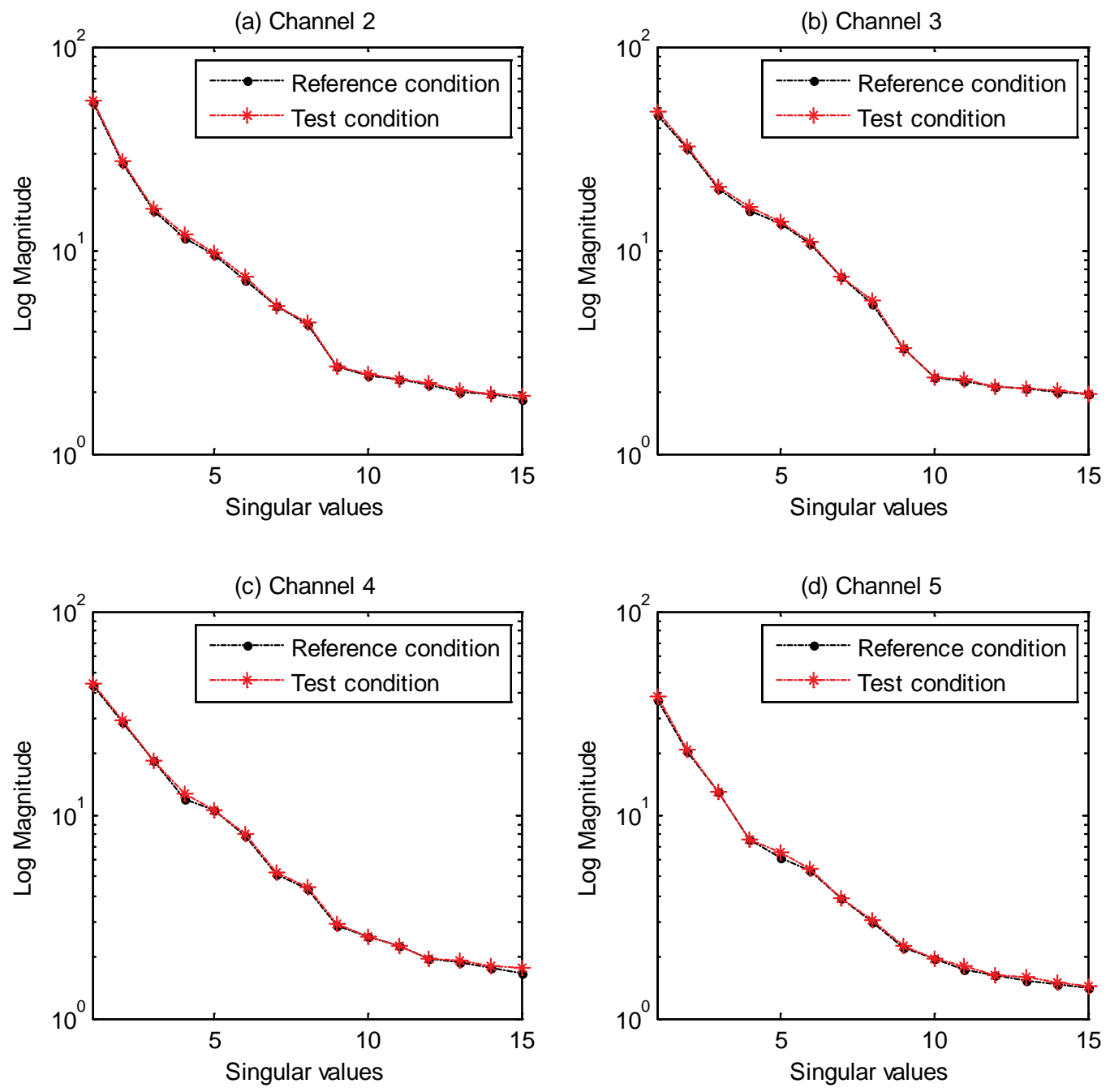

Figure 88: $\quad$ First 15 singular values in decreasing order for Channels 2-5 (State \#1). 
(a) Channel 2

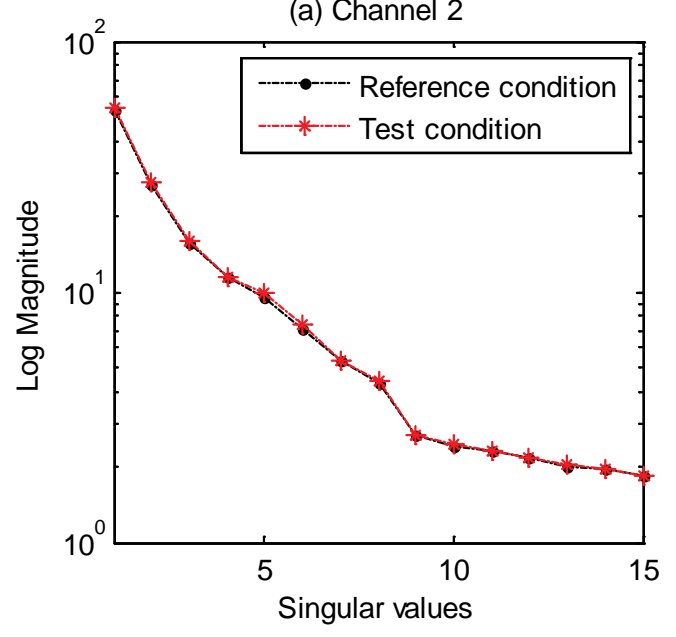

(c) Channel 4

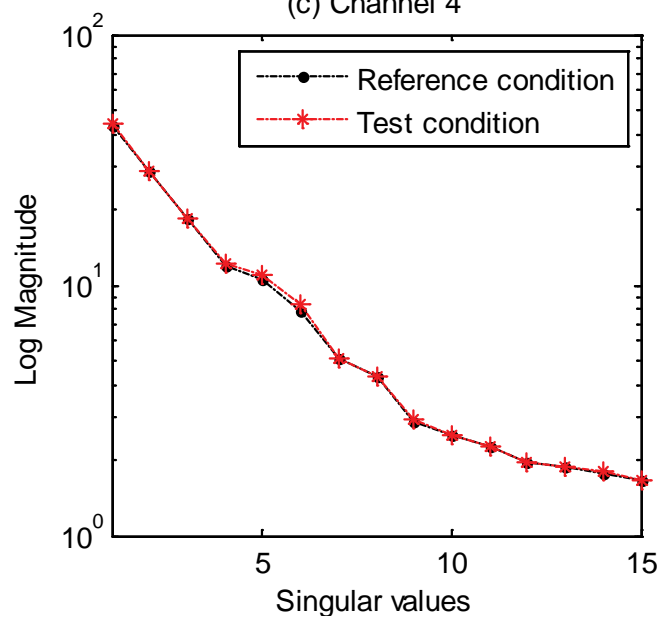

(b) Channel 3

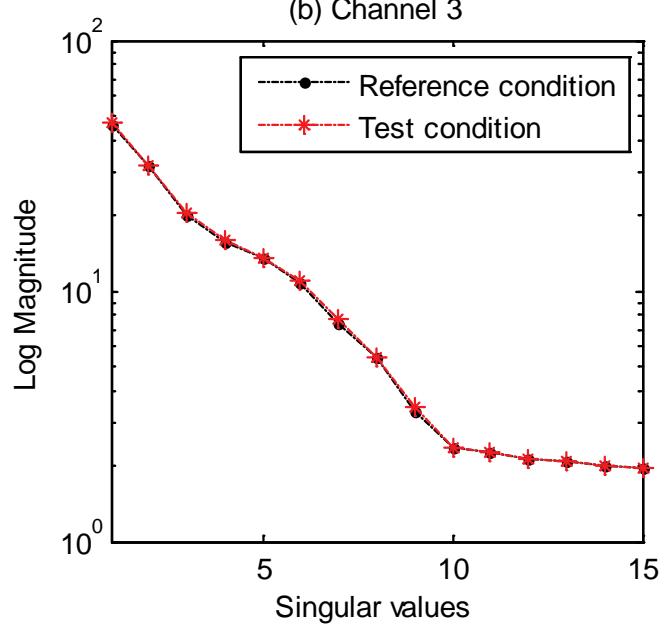

(d) Channel 5

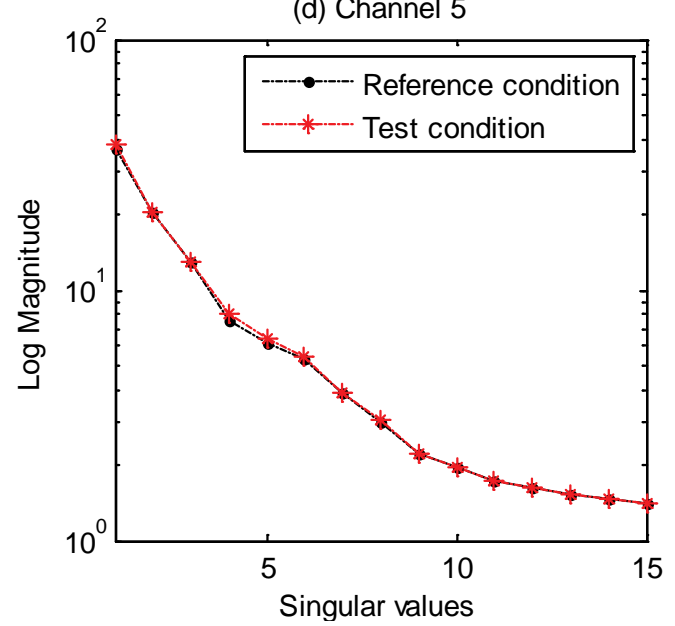

Figure 89: First 15 singular values in decreasing order for Channels 2-5 (State \#3). 
(a) Channel 2

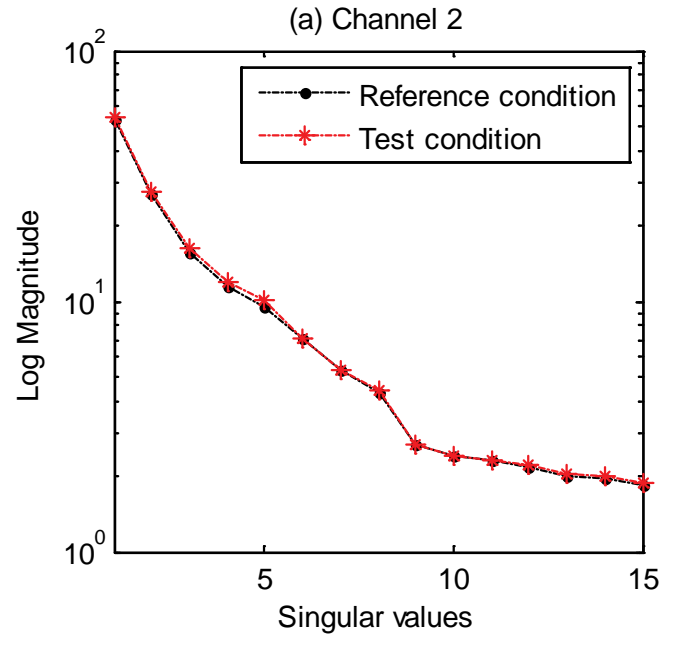

(c) Channel 4

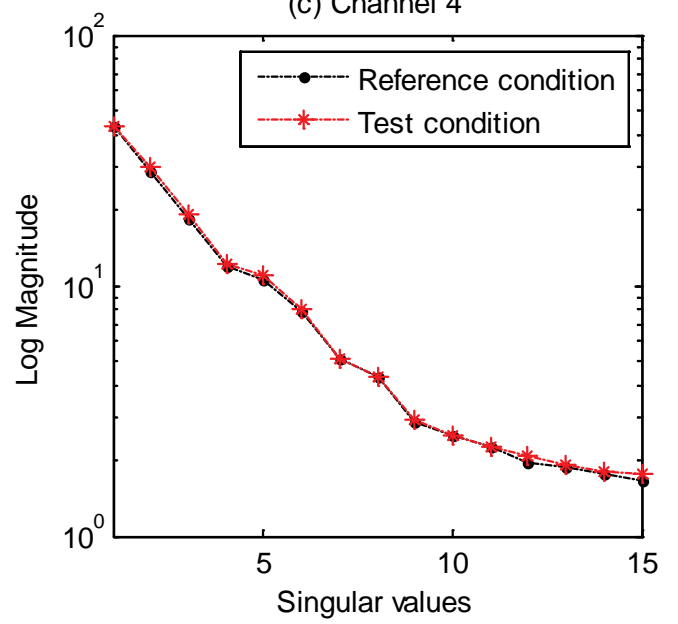

(b) Channel 3

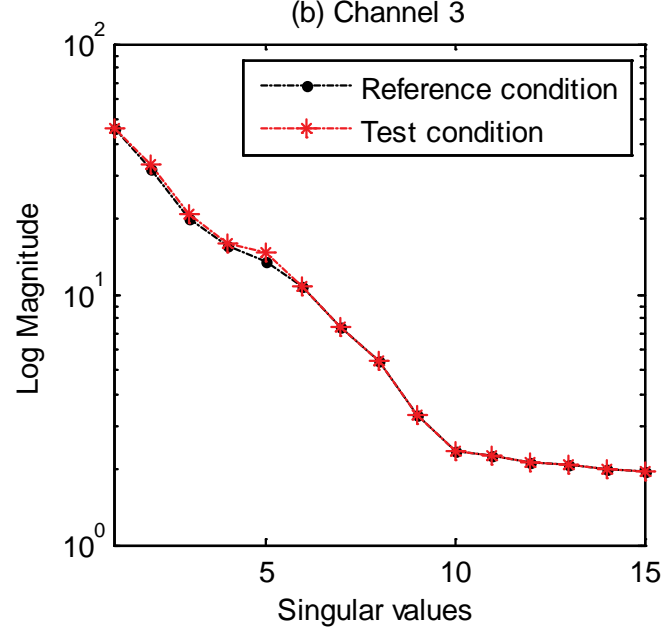

(d) Channel 5

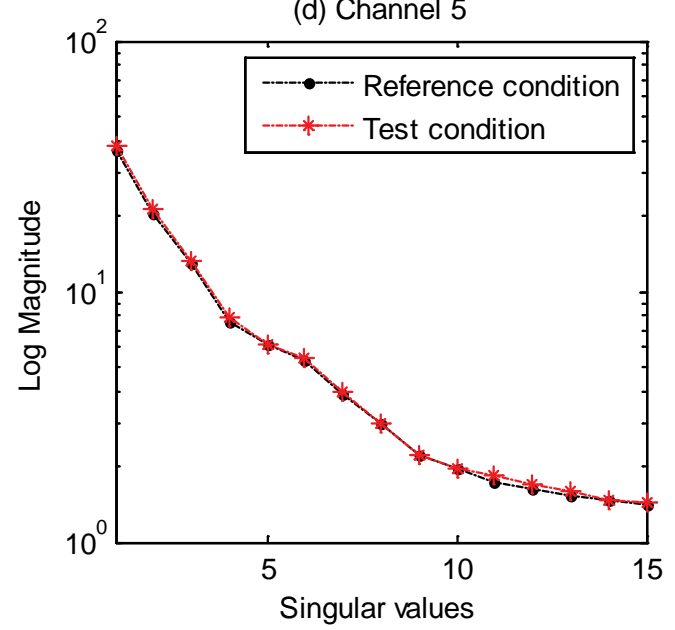

Figure 90: First 15 singular values in decreasing order for Channels 2-5 (State \#7). 
(a) Channel 2

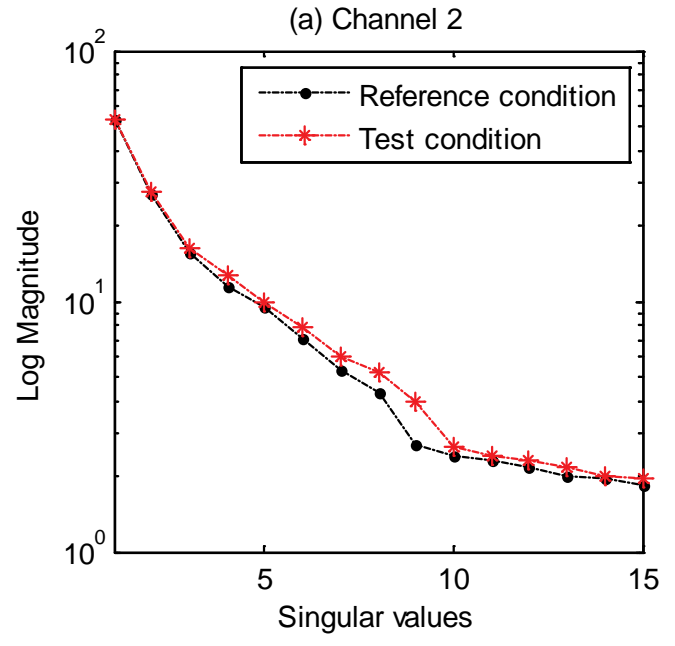

(c) Channel 4

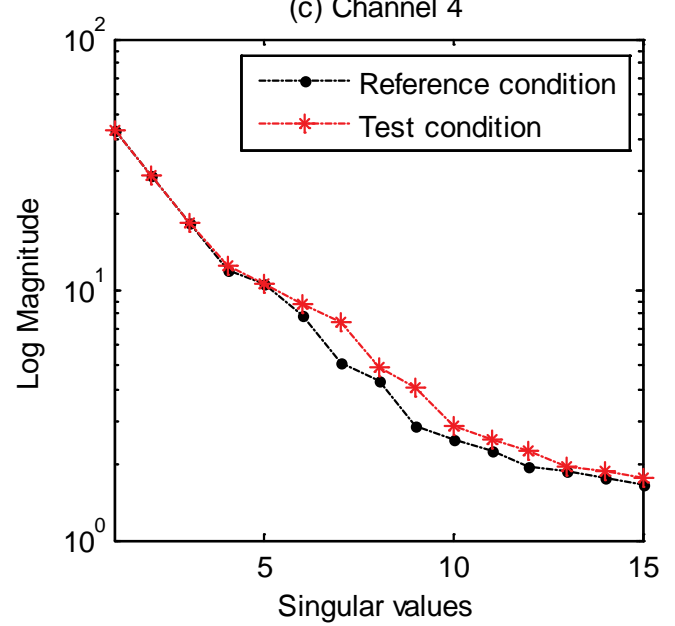

(b) Channel 3

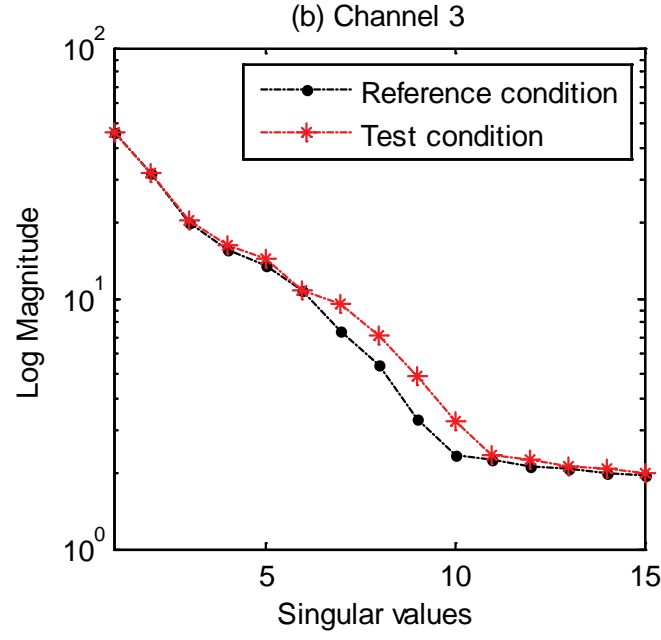

(d) Channel 5

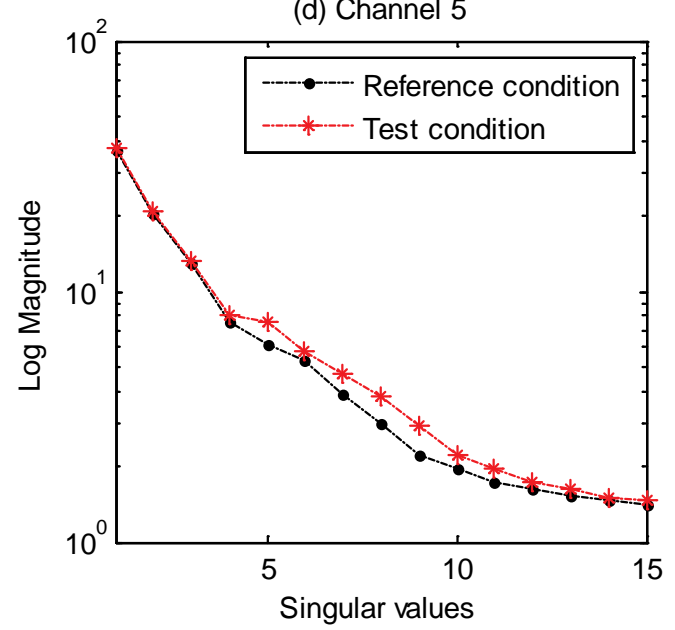

Figure 91: First 15 singular values in decreasing order for Channels 2-5 (State \#14). 
(a) Channel 2

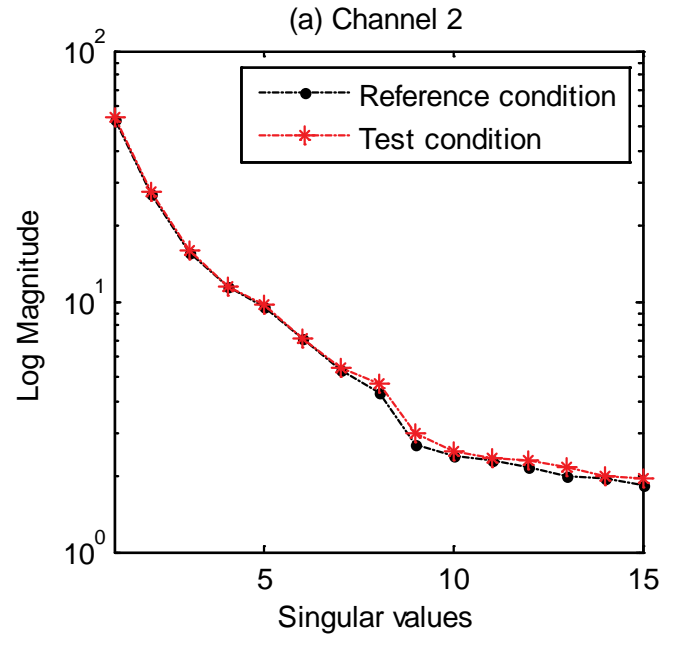

(c) Channel 4

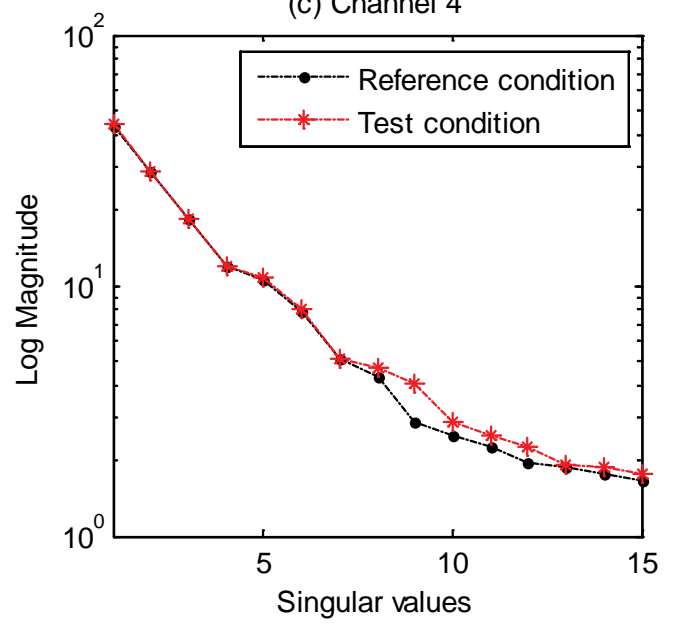

(b) Channel 3

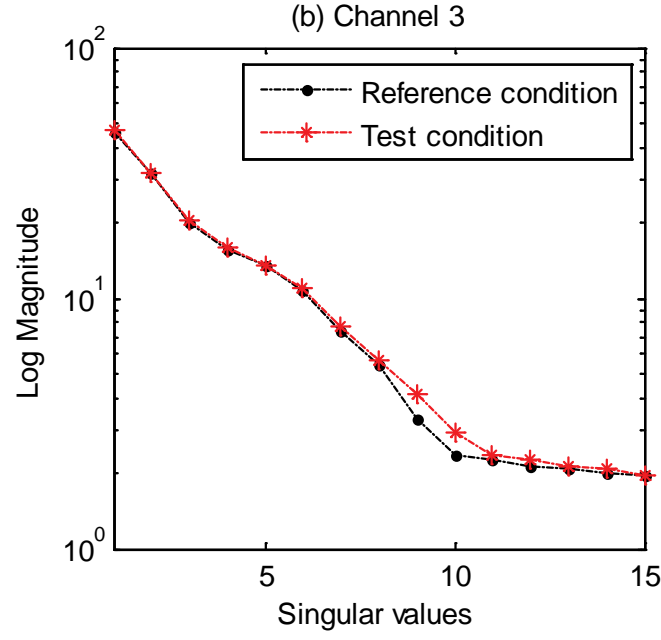

(d) Channel 5

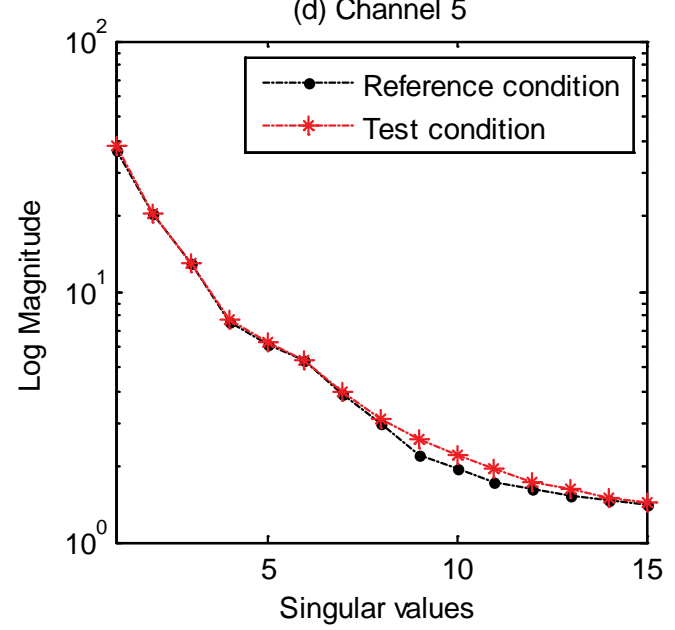

Figure 92: First 15 singular values in decreasing order for Channels 2-5 (State \#17). 


\section{CONCLUSIONS AND FINAL REMARKS}

The objective of this study was to apply the LANL statistical pattern recognition paradigm for SHM to data sets acquired from a laboratory three-story building structure. This report reviewed and applied various statistical procedures that have been widely used for dataanalysis problems in many different engineering fields.

In the hierarchical structure of damage detection, this report addressed the need for robust incipient damage-detection methods. Therefore, it is concerned with determining the existence of damage in the test structure. Even though locating and assessing the severity of damage is important in terms of estimating the residual lifetime of the structures, the reliable detection of damage existence must precede these more detailed damage descriptions. To achieve this objective, this report specially focused on developing statistical procedures addressing the feature extraction process and statistical modelling for feature classification in order to detect damage.

Before applying the statistical procedures to the data sets, a 4-DOF physics-based numerical model was developed to better understand the structural behavior of the system. The damping matrix for this model was obtained using the results of the experimental modal analysis. The Young's modulus was adjusted so that the analytically predicted natural frequencies agreed with the experimental ones. The developed numerical model assumes negligible friction between the rails and the structure. The model showed how to predict with a high level of certainty the measured baseline response.

In the feature extraction process, the AR models were subject to special attention. The estimated parameters as well as residual errors of the AR models were used as damage-sensitive features. The AR parameters' respective amplitudes decreased, accordingly, for smaller gaps between the bumper and suspended column, i.e., the smaller the gap, the smaller the amplitude of the AR parameters. Because the original data are highly correlated, the low-correlated AR residual errors were used in several statistical procedures for feature classification. Other damage-sensitive features were tested, such as modal parameters, statistical moments (mean, standard deviation, skewness, and kurtosis), PCA, STFT, WT, holder exponent, and correlation coefficients. Note that the correlation coefficients showed that both time histories and FRFs did not follow any deterministic pattern that can be picked out from the correlation. This result can be understood, based on the fact that the discontinuities associated with the damage caused shortduration changes to the system response and did not input any steady-state trends into the time histories.

The statistical modelling for feature classification was carried out by applying algorithms in an unsupervised learning mode. In the SPC techniques, the Shewhart X-bar control charts were employed in order to classify the mean value of grouped AR model residual errors from time histories corresponding to all the state conditions. This method showed that, in general, the number of outliers beyond the control limits increased for the damaged state conditions, even when they are affected by the simulated operational and environmental variations. Also it was shown that the feasibility of this method is a function of the AR model order. For this technique, the control limits were set up based on the distribution of the baseline condition.

The AANNs, Mahalanobis distance, and SVD algorithms were implemented in an unsupervised learning mode by first taking into account features from all the undamaged state conditions to train the algorithms. This procedure is currently defined as data normalization because it permits the algorithms to learn the underlying distribution of all the undamaged state conditions that included simulated operational and environmental variability introduced by changing the mass and stiffness of the structure. The Mahalanobis distance and SVD offered 
some advantages over AANNs in terms of computational effort. This fact would make those algorithms a better choice for implementation on embedded hardware.

In statistics, the data from a random process is said to be stationary and ergodic when the moments and joint moments (e.g., autocorrelation functions) are time invariant, and they do not change when computed over different sample records. Based on the analysis of those moments, it was assumed that the random process presented in this study is weakly stationary or stationary in the wide sense. However, the time-frequency analysis carried out using STFT and WT did show that the stiffness changes over time for the most damaged state. It turns out that the states do come from a nonstationary system. This contradiction can be related to the systematic impacts between the bumper and the column that make the test structure statistical stationary over an entire time record, but structurally nonstationary when shorter time scales are considered.

Significant contributions of this work include (i) understanding of how the AR model parameters change with the type of damage introduced by the bumper; (ii) comparison of different feature extractors and statistical procedures for feature classification using standard data sets; (iii) showing that even though a system can be statistically stationary, in reality it can be structurally nonstationary.

For future work, the authors recommend a deep investigation of the identification of the impacts over time. For this purpose, it is recommended to use sensors at impact locations. The impacts identified by accelerometer or acoustic sensors attached to the suspended column could be used to accurately identify the impacts and to correlate them with the other channel responses.

The data from this study and a detailed description of the test structure are available for download at http://institute.lanl.gov/ei/software-and-data/. 


\section{REFERENCES}

Bendat, J.S. and A.G. Piersol, Random Data: Analysis and Measurement Procedures, (John Wiley and Sons, Inc., Third Edition, 2000).

Bishop, C.M., Neural Networks for Pattern Recognition, (University Press, Oxford, 1995).

Box, G. and G. Jenkins, Time Series Analysis: Forecasting and Control, (Prentice Hall, Englewood Cliffs, New Jersey, 1976).

Box, G.E.P., G.M. Jenkins, and G.C. Reinsel, Time Series Analysis: Forecasting and Control, (Third Edition, Prentice-Hall, Inc., New Jersey, 1994).

Browman, A.W., "An Alternative Method of Cross-validation for the Smoothing of density estimates." Biometrika 71, (1984), pp. 352-360.

Chopra, A.K., Dynamics of Structures: Theory and Applications to Earthquakes Engineering, (Prentice Hall, Second Edition, 2001).

Doebling, S.W., C.R. Farrar, M.B. Prime, and D. Shevitz, "Damage Identification and Health Monitoring of Structural and Mechanical Systems from Changes in their Vibration Characteristics: A Literature Review," Los Alamos National Laboratory report: LA-13070-MS (1996).

Everitt, B.S., The Cambridge Dictionary of Statistics, (Second Edition, Cambridge University Press, 2002).

Farrar, C.R., P.J. Cornwell, S.W. Doebling, and M.B. Prime, "Structural Health Monitoring Studies of the Alamosa Canyon and I-40 Bridges," Los Alamos National Laboratory report: LA-13635-MS (2000).

Farrar, C.R., S.W. Doebling, and D.A. Nix, "Vibration-Based Structural Damage Identification." Philosophical Transactions of the Royal Society: Mathemathical, Physical \& Engineering Sciences 356(1778), pp. 131-149 (2001).

Farrar, C.R., H. Sohn and K. Worden, "Data Normalization: A Key to Structural Health Monitoring," in Proceedings of the Third International Structural Health Monitoring Workshop, (Stanford, California, DEStech Publications, Inc., Lancaster, Palo Alto, 2001) pp. 1229-1238.

Farrar, C.R., and K. Worden, "An Introduction to Structural Health Monitoring." Phil. Trans. R. Soc. A 365, pp. 303-315 (2007).

Figueiredo, E., A. Dimande, C. Félix, and J. Figueiras, "Instalation of a SHM System on the Pedestrian Bridge of Viana do Castelo," LABEST/FEUP Report, August (in Portuguese) (2007).

Grant, E.L. and R.S. Leavenworth, "Statistical Quality Control." McGraw-Hill Publishing Company, Sixth Edition (1988).

Gupta, B.C. and H.F. Walker, "Statistical Quality Control for the Six Sigma Green Belt." ASQ Quality Press, Milwaukee, Wisconsin (2007).

Ihn, J.B., and F.K. Chang, "Detection and Monitoring of Hidden Fatigue Crack Growth using a Built-in Piezoelectric Sensor/actuator Network: II. Validation using Riveted Joints and Repair Patches," Smart Materials and Structures 13(3) pp. 621-630 (2004).

Jolliffe, I.T., Principal Component Analysis, Springer, Second Edition (2004).

Kessler, S.S., S.M. Spearing and C. Soutis, "Damage Detection in Composite Materials using Lamb Wave Methods," Smart Materials and Structures 11(2), pp. 269-278 (2002). 
Kramer, M.A., "Nonlinear Principal Component Analysis using Autoassociative Neural Networks," AIChE Journal 37, No. 2, (February, 1991).

Kullaa, J., "Is Temperature Measurement Essential in Structural Health Monitoring?" Proceedings of the 4th International Workshop on Structural Health Monitoring, Stanford, California, September 15-17, Destech Publications Inc, pp. 717-724 (2003).

Kullaa, J., "Structural Health Monitoring under Variable Environmental or Operational Conditions," Proceedings of the Second Workshop on Structural Health Monitoring, Munich, Germany, July 7-9, Destech Publications Inc, pp. 1262-1269 (2005).

Maia, N.M.M. and J.M M. Silva, Theoretical and Experimental Modal Analysis, (John Wiley \& Sons Inc., NY,1997).

MATLAB, Statistics Toolbox, User's Guide, (The MathWorks, Inc., 2008) pp. 9.37-9.46.

Misiti, M., T. Misiti, G. Oppenheim, and J.M. Poggi, Wavelet Toolbox 4: User's Guide (The MathWorks, Inc., 2007).

Montgomery, D.C., Introduction to Statistical Quality Control (John Wiley \& Sons, Inc., New York, 1997).

Naeim, F., and J. Kelly, Design of Seismic Isolated Structures: From Theory to Practice (John Wiley and Sons, Inc., ISBN 0471149217, USA, 1999).

NIST/SEMATECH, "e-Handbook of Statistical Methods," http://www.itl.nist.gov/div898/handbook/, August 11, 2008.

Park, G., H. Sohn, C.R. Farrar, and D.J. Inman, "Overview of Piezoelectric Impedance-Based Health Monitoring and Path Forward," Shock and Vibration Digest 35(6), pp. 451-463 (2003).

Polikar, R., "The Wavelet Tutorial: The Engineer's Ultimate Guide to Wavelet Analysis," http://users.rowan.edu/ polikar/WAVELETS/WTtutorial.html, September 15 (2007).

Robertson, A.N., C.R. Farrar, and H. Sohn, "Singularity Detection for Structural Health Monitoring using Holder Exponents," Mechanical Systems and Signal Processing, 17(6), pp. 1163-1184 (2003).

Ruotolo, R., and C. Surace, "Damage Detection Using Singular Value Decomposition," DAMAS'97, University of Sheffield, United Kingdom, Sheffield Academic Press, June 30-2 July, pp. 87-96 (1997).

Ruotolo, R., and C. Surace, "Using SVD to Detect Damage in Structures with Different Operational Conditions," Journal of Sound and Vibration 226(3), pp. 425-439 (1999).

Rytter, A., "Vibration Based Inspection of Civil Enginnering Structures." Ph.D. Dissertation, Department of Building Technology and Structural Engineering, Alborg University, Denmark (1993).

Silverman, B.W., Density Estimation for Statistics and Data Analysis, Chapman and Hall, New York (1986).

Sohn, H., "Effects of Environmental and Operational Variability on Structural Health Monitoring," Phil. Trans. R. Soc. A 365, pp. 539-560 (2006).

Sohn, H., C. Farrar, N. Hunter, and K. Worden, "Applying the LANL Statistical Pattern Recognition Paradigm for Structural Health Monitoring to Data from a Surface-Effect Fast Patrol Boat," Los Alamos National Laboratory Report LA-13761-MS (2001). 
Sohn, H., C.R. Farrar, F.M. Hemez, D.D. Shunk, and D.W. Stinemates, "A Review of Structural Health Monitoring Literature from 1996-2001," Los Alamos National Laboratory report: LA-13976-MS (2004).

Sohn, H., G. Park, J.R. Wait, N. Limback, and C.R. Farrar, "Wavelet-Based Signal Processing for Detecting Delamination in Composite Plates," Smart Materials and Structurals 13(1), pp. 153-160 (2004).

Sohn, H., K. Worden, and C.R. Farrar, "Novelty Detection under Changing Environmental Conditions," SPIE's 8th Annual International Symposium on Smart Structures and Materials, Newport Beach, California, March 4-8 (2001).

Sohn, H., K. Worden, and C.R. Farrar, "Statistical Damage Classification under Changing Environmental and Operational Conditions," Journal of Intelligent Materials Systems and Structures 13, No. 9, pp. 561-574 (2003).

Sohn, H., M. Dzwonczyk, E.G. Straser, A.S. Kiremidjian, K.H. Law, and T. Meng, "An Experimental Study of Temperature Effects on Modal Parameters of the Alamosa Canyon Bridge," Earthquake Engineering and Structural Dynamics 28, pp. 879-897 (1999).

Wirsching, P.H., T.L. Paez, and H. Ortiz, Random Vibrations: Theory and Practice, (John Wiley \& Sons, Inc., 1995).

Woon, C.E. and L.D. Mitchell, "Variations in Structural Dynamic Charateristics Caused by Changes in Ambient Temperature: I. Experimental," Proceedings of the 14th International Modal Analysis Conference, February 12-15 (1996).

Worden, K., and J.M. Dulieu-Barton, "An Overview of Intelligent Fault Detection in Systems and Structures," International Journal of Structural Health Monitoring 3(1), pp. 85-98 (2004).

Worden, K., C.R. Farrar, G. Manson, and G. Park, "The Fundamental Axioms of Structural Health Monitoring," Proceedings of the Royal Society 463, pp. 1639-1664 (2007).

Worden, K., and G. Manson, "Damage Detection using Outlier Analysis," Journal of Sound and Vibration 229(3), pp.647-667 (2000).

Worden, K., H. Sohn, and C.R. Farrar, "Novelty Detection in a Changing Environmental: Regression and Interpolation Approaches, " Journal of Sound and Vibration 258(4), pp.741-761 (2002). 
This report has been reproduced directly from the best available copy. It is available electronically on the Web (http://www.doe.gov/bridge).

Copies are available for sale to U.S. Department of Energy employees and contractors from:

Office of Scientific and Technical Information

P.O. Box 62

Oak Ridge, TN 37831

(865) 576-8401

Copies are available for sale to the public from: National Technical Information Service

U.S. Department of Commerce

5285 Port Royal Road

Springfield, VA 22161

(800) 553-6847 
- Los Alamos NATIONAL LABORATORY

EST.1943 